\title{
Visible-Light-Induced 1,4-hydroxysulfonylation of Vinyl Enynes with Sulfonyl Chlorides: the Bridge of Chloride Linking Water and Enynes
}

Cheng-Yun Zhang, ${ }^{[a]}$ Jie Zhu, ${ }^{*[a]}$ Su-Hang Cui, ${ }^{[a]}$ Xiao-Yu Xie, ${ }^{[a]}$ XiaoDong Wang, ${ }^{[a]}$ and Lei $\mathrm{Wu}^{*[a, b]}$

${ }^{[a]}$ Jiangsu Key Laboratory of Pesticide Science and Department of Chemistry, College of Sciences, Nanjing Agricultural University, Nanjing 210095, P. R. China. E-mail: zhujie@njau.edu.cn; rickywu@njau.edu.cn.

${ }^{[b]}$ College of Chemical Engineering, Xinjiang Agricultural University, Urumqi 830052, P. R. China 
Table of Contents for Supporting Information

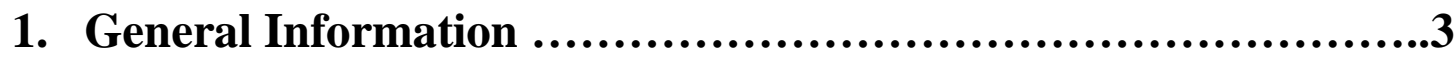

2. General Procedures for Substrates Preparation ...................4

3. General Procedures for Visible-Light-Induced 1,4-hydroxysulfonylation of Vinyl Enynes with Sulfonyl Chlorides.........................5

4. Optimization of the Reaction Conditions..........................6

5. The Role of the Vinyl Moiety on Reigo-selectivity.................10

6. Control Experiments and Mechanistic Studies....................11

7. Detection and Capture of Hydroxyl Radical.......................13

8. Synthetic Application.......................................16

9. X-Ray Crystallography Data of Compound 3aa .................18

10. X-Ray Crystallography Data of Compound 4.....................26

11. Characterizations of Substrates and Products......................34

12. ${ }^{1} \mathrm{H}$-NMR, ${ }^{13} \mathrm{C}$-NMR, ${ }^{19} \mathrm{~F}$-NMR, ${ }^{31} \mathrm{P}$-NMR and HRMS Spectra for Substrates and Products.............................................68 


\section{General Information}

Solvents and reagents were reagent grade and used without purification unless otherwise noted. Anhydrous dichloromethane and methanol were bought from Aladdin Chemicals, Shanghai, China. Photochemical reactions were carried out with blue LED made by JiaDeng ( $\lambda=450-455 \mathrm{~nm}, 15 \mathrm{~W}$ ) which was purchased online at website of https://shop358870690.taobao.com/?spm=a230r.7195193.1997079397.2. $11734595 \mathrm{RntKz} 9$. For irradiation, the material of the reaction vessel purchased from Synthware is common glass; the distance from the light source is about $2.0 \mathrm{~cm}$. No filters were used in the general procedures. Column chromatography was performed using silica gel (200-300 mesh). All ${ }^{1} \mathrm{H}-\mathrm{NMR}$ (400 MHz or $600 \mathrm{MHz}$ ) spectra were recorded on a BrukerDMX 400 or 600 using $\mathrm{CDCl}_{3}$ solution in the presence of tetramethylsilane (TMS) as an internal standard and are reported in ppm $(\delta)$. Coupling constants are reported in Hertz $(\mathrm{Hz})$. Spectral splitting patterns are designated as s, singlet; d, doublet; t, triplet; q, quartet; p, pentet; m, multiplet; and br, broad. High resolution mass spectroscopic data of the products were collected on a Waters Micromass GCT instrument using EI $(70 \mathrm{eV})$ or an Agilent Technologies 6540 UHD Accurate-Mass Q-TOF LC/MS using ESI. Electron paramagnetic resonance (EPR) spectra were recorded at room temperature using a Bruker A300 spectrometer. Singlecrystal X-ray diffraction analysis of 3aa and $\mathbf{4}$ was carried out on a Bruker D8 QUEST diffractometer with microfocus Ius 2.0 Mo-Ka radiation $(\lambda=0.71073 \AA)$ at $296(2) \mathrm{K}$. 


\section{General Procedures for Substrates Preparation}
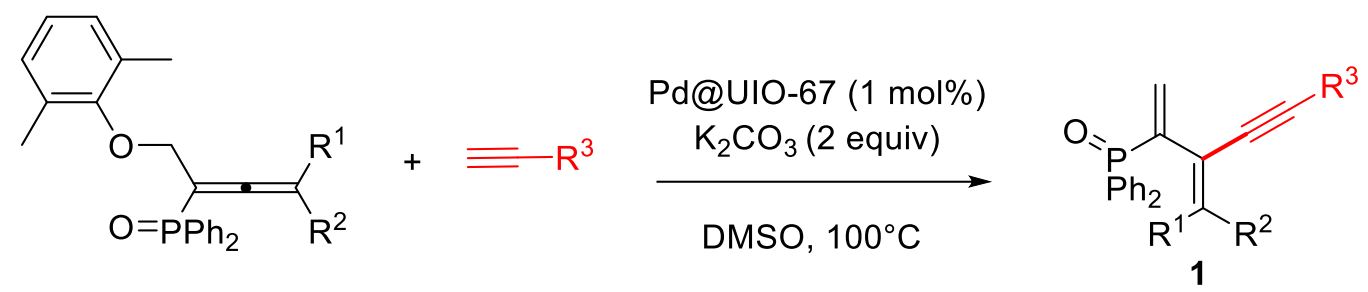

The vinyl enyne substrates (1) were prepared according to a procedure reported in our previous work, please see more details in: X.-D. Wang, J.J. Wu, X. Sun, W.-C. Yang, J. Zhu, L. Wu, Adv. Synth. Catal. 2018, 360, 3518-3525.

To a $10 \mathrm{~mL}$ vial was added allenylphosphine oxides $(0.3 \mathrm{mmol})$, alkynes $(0.6 \mathrm{mmol})$, potassium carbonate $(0.6 \mathrm{mmol})$, DMSO $(3 \mathrm{~mL})$ and Pd@UIO-67 (1 mol\%, $30 \mathrm{mg})$, respectively. The reaction was then allowed to react at $100{ }^{\circ} \mathrm{C}$ in an oil bath for a certain time until the complete consuming of starting materials monitored by TLC. The reaction mixture was extracted with EtOAc $(1 \mathrm{~mL} \times 3)$. The combined organic extract was washed with brine and dried over anhydrous $\mathrm{Na}_{2} \mathrm{SO}_{4}$. The solvent was evaporated under reduced pressure and the residue was purified by column chromatography on silica gel using petroleum ether/ethylacetate (1:1) as the eluent to afford the vinyl enynes (1). After being finished, the purity and structure of compounds were confirmed by ${ }^{1} \mathrm{H}-\mathrm{NMR}$. 


\section{General Procedures for Visible-Light-Induced 1,4-hydroxy-}

sulfonylation of Vinyl Enynes with Sulfonyl Chlorides
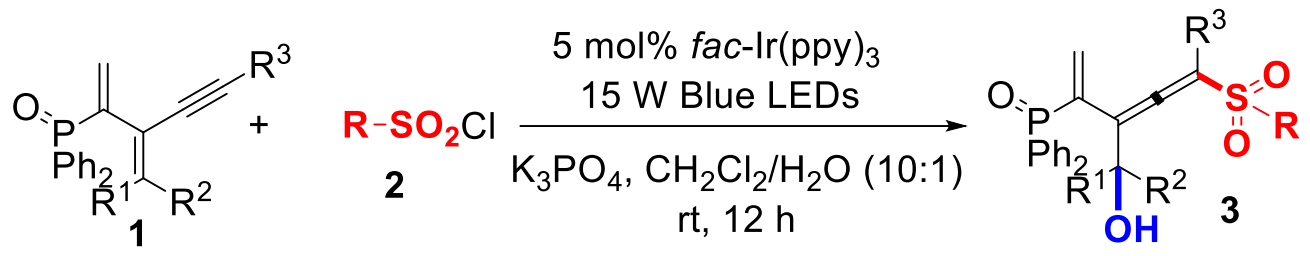

To a $10 \mathrm{~mL}$ vial was added vinyl enyne ( $0.2 \mathrm{mmol}, 1.0$ equiv), sulfonyl chloride (0.5 mmol, 2.5 equiv. $4 \mathrm{H}), f a c-\operatorname{Ir}(\mathrm{ppy})_{3}(5.0 \mathrm{~mol} \%)$ and potassium phosphate ( $0.4 \mathrm{mmol}, 2$ equiv.). The reaction flask was sealed, evacuated and back-filled with argon for three times. Then $2 \mathrm{~mL}$ degassed solvent $\left(\mathrm{CH}_{2} \mathrm{Cl}_{2} /\right.$ water $)(v: v=10: 1)$ was injected into the sealed vial. The reaction mixture was stirred under the irradiation of $15 \mathrm{~W}$ blue LEDs (450$455 \mathrm{~nm}$ ) at room temperature for 12 hours. Upon completion of the reaction, the solvent was evaporated under reduced pressure and the residue was purified by column chromatography on silica gel using petroleum ether/ethylacetate $(2.5: 1)$ to afford the target product $(\mathbf{3})$. 


\section{Optimization of the Reaction Conditions}

Table S1. Screening of Photocatalysts ${ }^{a}$

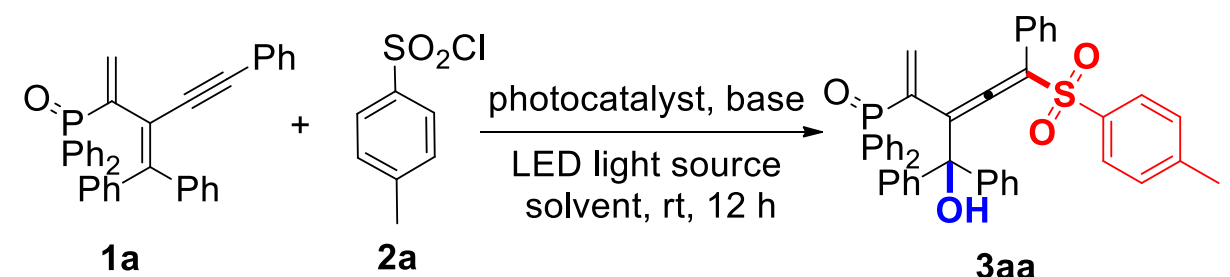

\begin{tabular}{|c|c|c|}
\hline entry & photocatalyst & yield $(\%)^{\mathrm{c}}$ \\
\hline 1 & $f a c-\operatorname{Ir}(\mathrm{ppy})_{3}$ & 67 \\
\hline 2 & 4CzIPN & 53 \\
\hline 3 & $\operatorname{eosin} \mathrm{Y}$ & 11 \\
\hline 4 & 9-Mesityl-10-methylacridinium Perchlorate & 13 \\
\hline 5 & acid Red 94 & N.R. \\
\hline 6 & Riboflavin & 11 \\
\hline 7 & $\mathrm{CuCl}$ & N.R. \\
\hline 8 & Rhodamine B & N.R. \\
\hline 9 & Acid Blue 93 & N.R. \\
\hline 10 & {$\left[\operatorname{Ir}(\mathrm{dtbbpy})(\mathrm{ppy})_{2}\right]\left[\mathrm{PF}_{6}\right]^{\mathrm{b}}$} & 88 \\
\hline 11 & $f a c-\operatorname{Ir}(\mathrm{ppy}) 3^{\mathrm{b}}$ & $>95(81)$ \\
\hline 12 & $4 \mathrm{CzIPN}^{\mathrm{b}}$ & 78 \\
\hline $13^{\mathrm{d}}$ & $f a c-\operatorname{Ir}(\mathrm{ppy}) 3^{\mathrm{b}}$ & N.R. \\
\hline 14 & - & N.R. \\
\hline $15^{\mathrm{d}}$ & $f a c-\operatorname{Ir}(\text { ppy })_{3}^{\mathrm{b}, \mathrm{e}}$ & N.R. \\
\hline
\end{tabular}

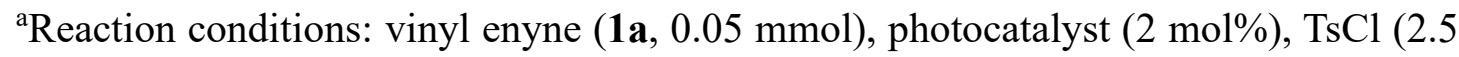
equiv.), $\mathrm{K}_{3} \mathrm{PO}_{4}$ (2.0 equiv.), $\mathrm{CH}_{2} \mathrm{Cl}_{2} / \mathrm{H}_{2} \mathrm{O}=10: 1$ ( $2 \mathrm{~mL}$ ). The reaction mixture was then stirred for 12 hours irradiated by $15 \mathrm{~W}$ Blue LEDs at room temperature under argon atmosphere. ${ }^{b}$ photocatalyst (5 mol\%). ${ }^{~}$ Yields based on ${ }^{31} \mathrm{P}$ NMR, isolated yields in

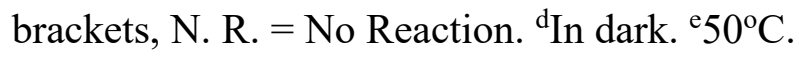


Table S2. Screening of solvents ${ }^{\mathrm{a}}$

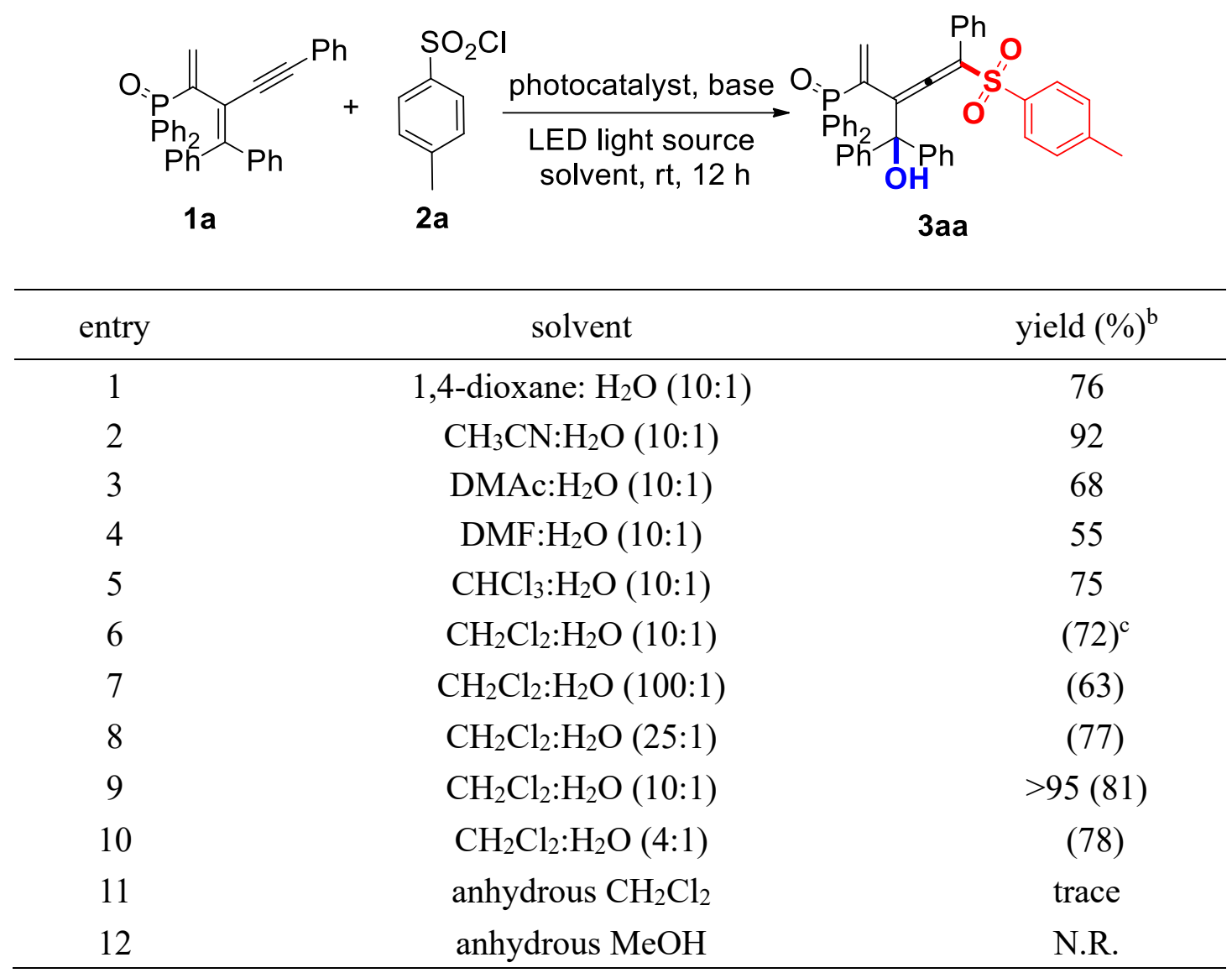

${ }^{\text {a}}$ Reaction conditions: vinyl enyne (1a, $\left.0.2 \mathrm{mmol}\right)$, fac-Ir(ppy) 3 (5 mol\%), TsCl $(2.5$ equiv.), $\mathrm{K}_{3} \mathrm{PO}_{4}$ (2.0 equiv.), $2 \mathrm{~mL}$ solvent. The reaction mixture was then stirred for 12 hours irradiated by $15 \mathrm{~W}$ Blue LEDs at room temperature under argon atmosphere. ${ }^{\text {b}}$ Yields based on ${ }^{31} \mathrm{P}$ NMR, isolated yields in brackets. ${ }^{\mathrm{c}} 1 \mathrm{~mL}$ solvent. 
Table S3. Screening of Bases ${ }^{\mathrm{a}}$

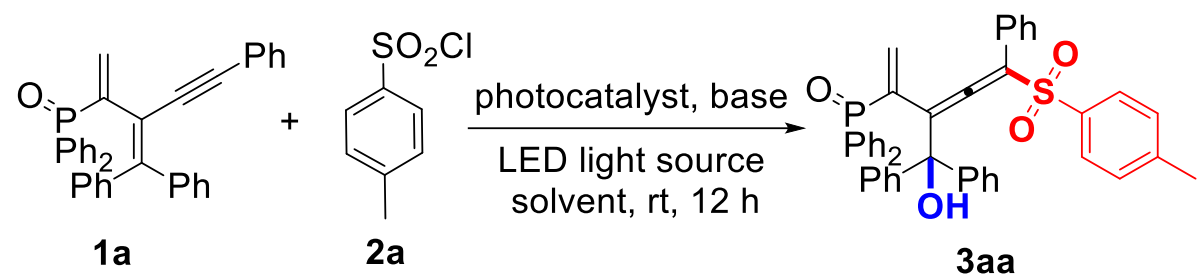

\begin{tabular}{ccc}
\hline entry & base & yield $(\%)^{\mathrm{b}}$ \\
\hline 1 & triethylamine & $(51)$ \\
2 & $\mathrm{DBU}$ & $(72)$ \\
3 & $\mathrm{Na}_{2} \mathrm{CO}_{3}$ & $(66)$ \\
4 & $\mathrm{~K}_{3} \mathrm{PO}_{4}$ & $>95(81)$ \\
5 & $\mathrm{~K}_{2} \mathrm{HPO}_{4}$ & $(65)$ \\
6 & - & $(36)$ \\
\hline
\end{tabular}

${ }^{\text {a}}$ Reaction conditions: enyne (1a, $\left.0.2 \mathrm{mmol}\right)$, fac- $\operatorname{Ir}(\mathrm{ppy})_{3}$ (5 mol\%), TsCl (2.5 equiv.), base (2.0 equiv.), $\mathrm{CH}_{2} \mathrm{Cl}_{2} / \mathrm{H}_{2} \mathrm{O}=10: 1(2 \mathrm{~mL})$. The reaction mixture was then stirred for 12 hours irradiated by $15 \mathrm{~W}$ Blue LEDs at room temperature under argon atmosphere.

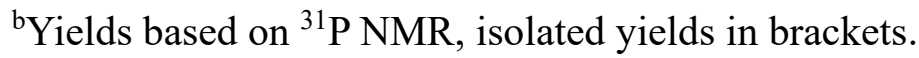


Table S4. Screening of Light Source, Reaction Time and Substrate/Base Ratios ${ }^{\mathrm{a}}$

endight source
solvent, rt, $12 \mathrm{~h}$

${ }^{a}$ Reaction conditions: enyne (1a, $\left.0.2 \mathrm{mmol}\right)$, fac- $\mathrm{Ir}(\mathrm{ppy})_{3}(5 \mathrm{~mol} \%), \mathrm{CH}_{2} \mathrm{Cl}_{2} / \mathrm{H}_{2} \mathrm{O}=10: 1$ $(2 \mathrm{~mL})$. The reaction mixture was then stirred for 12 hours irradiated by LEDs at room temperature under argon atmosphere. ${ }^{\text {b}}$ Yields based on ${ }^{31} \mathrm{P}$ NMR, isolated yields in brackets. ${ }^{c}$ Reaction time: 9 hours. ${ }^{\mathrm{d}}$ Reaction time: 24 hours. 


\section{The Role of Vinyl Moiety on Regio-selectivity}

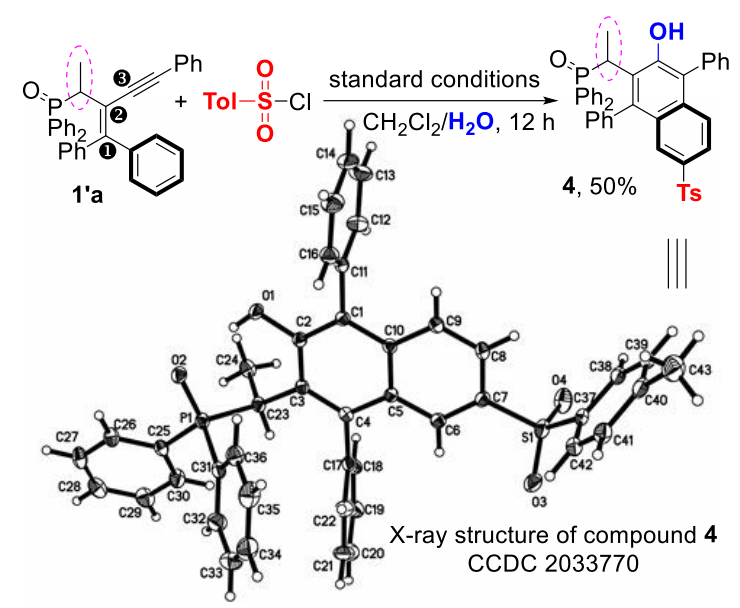

In order to clarify the critical role of vinyl moiety on regio-selectivity, a vinyl-free analogue (1'a) and tosyl chloride were treated with the optimal conditions. Although the photocatalytic hydroxylsulfonylation occurred as well, the regioselectivity was entirely switched, giving a highly-substituted $\beta$-naphthol derivative (4) in 50\% isolated yield. The X-ray structure of $\mathbf{4}$ (CCDC 2033770) presented that the hydroxylation performs at alkyne moiety instead of alkene moiety, and the cascade tosylation occurs on a distal phenyl group.

Procedure for reaction with enyne substrate 1'a instead of vinyl enyne: To a $10 \mathrm{~mL}$ vial was added enyne substrate 1'a (0.2 mmol, 1.0 equiv.), sulfonyl chloride (0.5 mmol, 2.5 equiv.), $f a c-\operatorname{Ir}(\mathrm{ppy})_{3}(5.0 \mathrm{~mol} \%)$ and potassium phosphate $(0.4 \mathrm{mmol}, 2$ equiv.). The reaction flask was sealed, evacuated and back-filled with argon for three times. Then $2 \mathrm{~mL}$ degassed solvent $\left(\mathrm{CH}_{2} \mathrm{Cl}_{2} /\right.$ water $)(v: v=10: 1)$ was injected into the sealed vial. The reaction mixture was stirred under the irradiation of $15 \mathrm{~W}$ blue LEDs at room temperature for 12 hours. Upon completion of the reaction, the solvent was evaporated under reduced pressure and the residue was purified by column chromatography on silica gel using petroleum ether/ ethyl acetate (2.5:1) to afford the target product (4) in 50\% yield $(67.9 \mathrm{mg})$. 


\section{Control Experiments and Mechanistic Studies}

eq. a: Radical Inhibition Experiment<smiles>C=C(C(C#C[Se]c1ccccc1)=C(c1ccccc1)c1ccccc1)P(=O)(O)c1ccccc1</smiles>

$1 \mathrm{a}$<smiles>[3H]C(=C=C(C(=C)[PH](=O)c1ccccc1)C(O)(c1ccccc1)c1ccccc1)c1ccccc1</smiles>

3aa, $0 \%$

eq. b: Radical Intermediates Caputure

$1 a$<smiles>C=CCN(CC=C)C(=O)c1ccccc1</smiles>

2a<smiles>C=C(C#CC(=O)P(=O)(c1ccccc1)c1ccccc1)C(O)(c1ccccc1)c1ccccc1</smiles>

HR-MS

Standard Conditions

$\mathrm{CH}_{2} \mathrm{Cl}_{2} / \mathrm{H}_{2} \mathrm{O}$
Ts<smiles>CC1CNCC1C</smiles><smiles>CC(=O)C(=O)c1ccccc1</smiles>

$[\mathrm{M}+\mathrm{H}]^{+}: 358.1462$

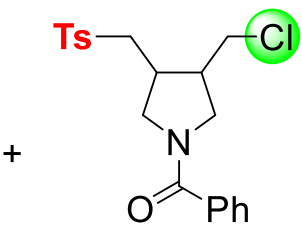

$[\mathrm{M}+\mathrm{H}]^{+}: 392.1071$ $[\mathrm{M}+\mathrm{Na}]^{+}: 414.0890$

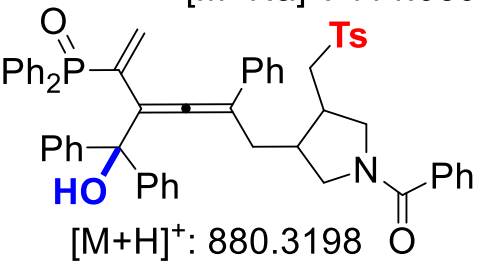

$[\mathrm{M}+\mathrm{Na}]^{+}: 902.3013$

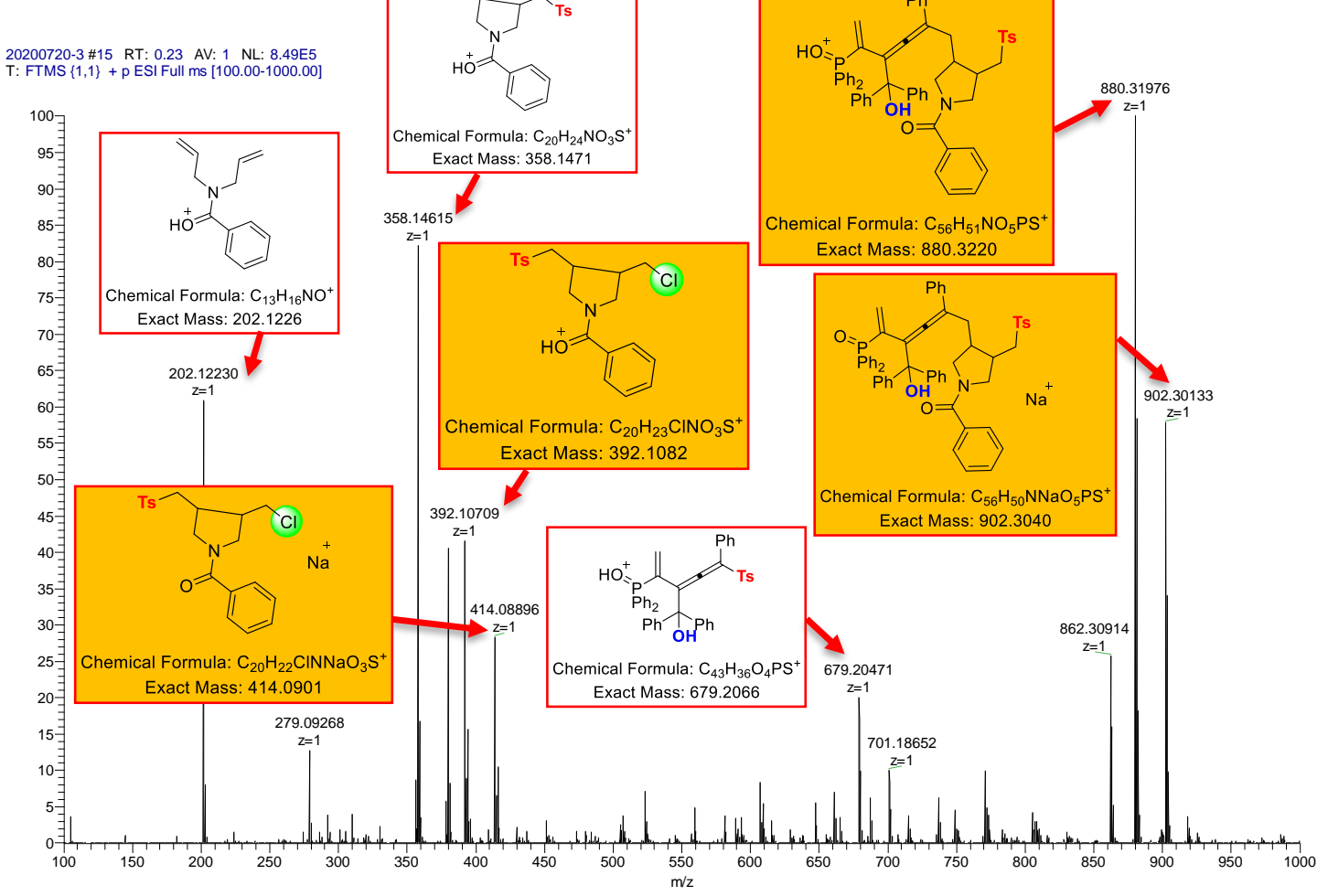


eq. c: Oxygen Isotopic Labelling<smiles>C=C(C(=O)P)C(C#Cc1ccccc1)=C(c1ccccc1)c1ccccc1</smiles><smiles>C=C(P=O)C(=C=C([As])Pc1ccccc1)C(c1ccccc1)(P(O)c1ccccc1)[PH](=O)c1ccccc1</smiles>

$1 \mathrm{a}$

3aa-O ${ }^{18}$, detected by HR-MS

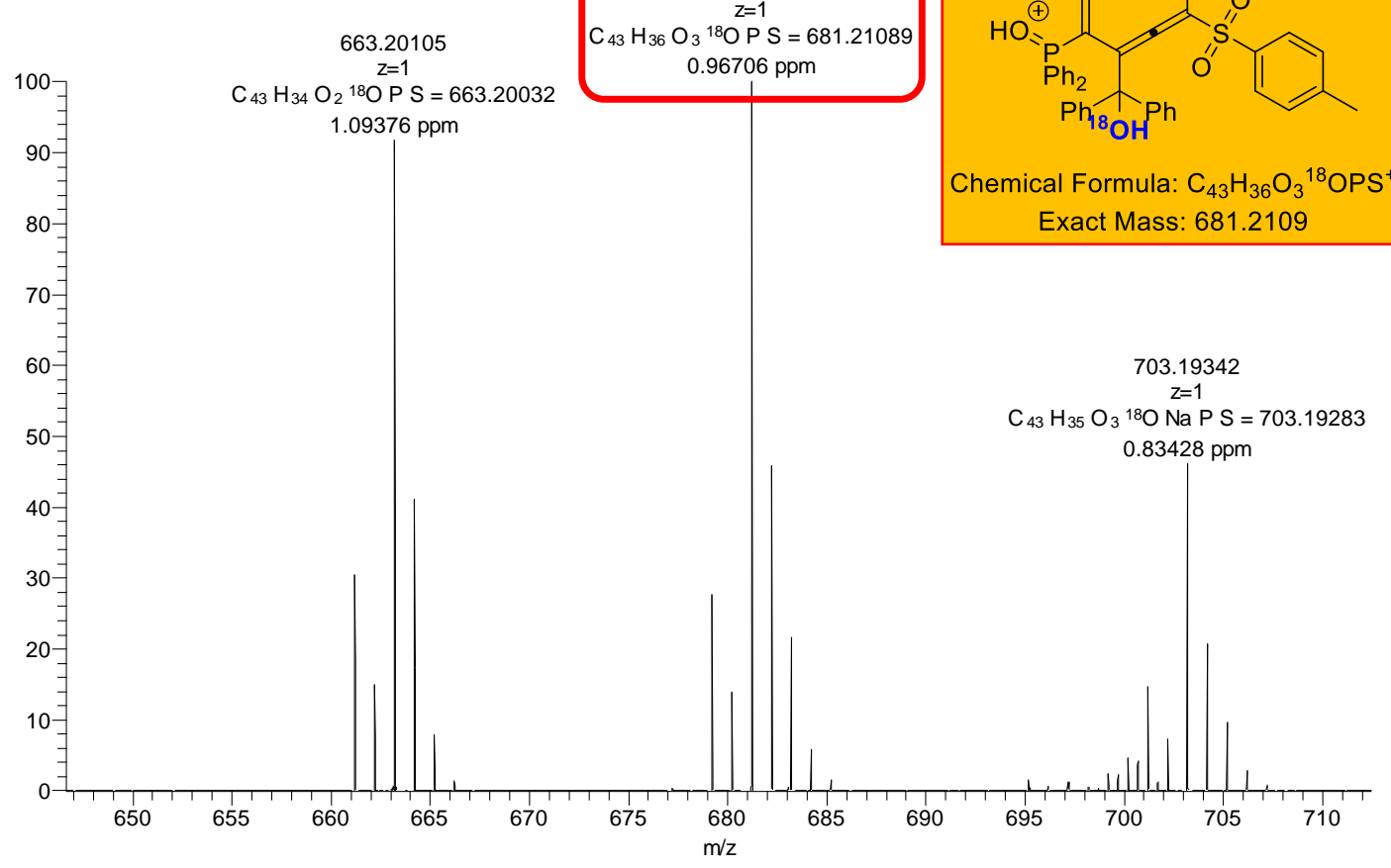

eq. d: Control Experiment under Chloride-free Conditions

$$
\begin{aligned}
& 1 \mathrm{a}+\mathrm{TsNa} \stackrel{\text { standard conditions }}{\longrightarrow} \underline{\mathbf{3 a a}}, \underline{0 \%} \\
& 1 \mathrm{a}+\mathrm{TsNHNH}_{2} \stackrel{\text { standard conditions }}{\longrightarrow} \underline{\mathbf{3 a a}, 0 \%}
\end{aligned}
$$

eq. e: Parallel Experiment with Chloride Radical Source

$$
1 \mathrm{a}+\mathrm{TsNa}+\mathrm{NCS} \stackrel{\text { standard conditions }}{\longrightarrow} \underline{\mathbf{3 a a}}, \underline{49 \%}
$$




\section{Detection and Capture of Hydroxyl Radical}

\subsection{EPR experiment}

The EPR signals were acquired at 90 seconds after mixing the reactants and the radical trapping agents. When the 5,5-dimethyl-1pyrroline-N-oxide (DMPO) was used as capture agents, the DMPO trapped - $\mathrm{OH}$ were detected, while no signal was observed in dark. Standard condition: DMPO $(0.6 \mathrm{mmol}), 1 \mathrm{a}(0.2 \mathrm{mmol}), \mathrm{TsCl}(0.5 \mathrm{mmol}), \mathrm{K}_{3} \mathrm{PO}_{4}$ (0.4 mmol), fac- $\operatorname{Ir}\left(\mathrm{ppy}_{3}\right)(5 \mathrm{~mol} \%), \mathrm{DCM} / \mathrm{H}_{2} \mathrm{O}(10: 1,2 \mathrm{~mL})$ and $15 \mathrm{~W}$ blue LED.

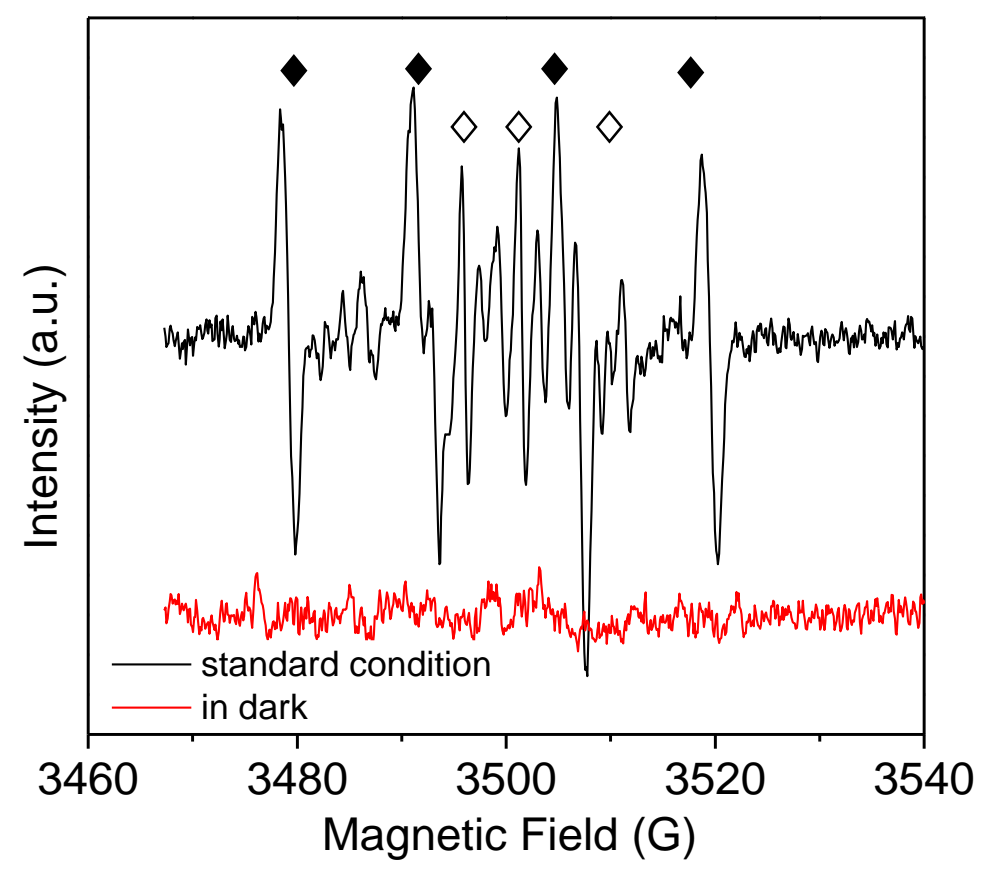

Figure S1. EPR signals of DMPO trapped $\bullet \mathrm{OH}(\diamond)$. The diamond symbol $(\diamond)$ indicates the adduct of DMPO and alkyl or chlorine radical that might be caused by DMPO impurities. 


\subsection{Fluorescence Sensing Experiment}

According to the Liu's work, dihydroquinoline $\mathbf{6}$ was used as probe to detect the hydroxyl radicals. The fluorescence image demonstrated obvious color change due to the generation of $\bullet \mathrm{OH}$, and the HR-MS showed that $\mathbf{6}$ was almost completely consumed, and the product $\mathbf{6}$ ' caused by the hydroxyl radicals was detected. Standard condition: $1 \mathbf{a}(0.2 \mathrm{mmol})$, $\mathrm{TsCl}(0.5 \mathrm{mmol}), \mathrm{K}_{3} \mathrm{PO}_{4}(0.4 \mathrm{mmol}), f a c-\operatorname{Ir}\left(\mathrm{ppy}_{3}\right)(5 \mathrm{~mol} \%), \mathrm{DCM} / \mathrm{H}_{2} \mathrm{O}$ (10:1, 2mL) and 15W blue LED. Reference: Wu, Y.; Huang, W.; Peng, D.; Huang, X.; Gu, S.; Wu, S.; Deng, T.; Liu, F. Synthesis of Dihydroquinolines as Scaffolds for Fluorescence Sensing of Hydroxyl Radical. Org. Lett. 2021, 23, 135-139.<smiles>CC1=CN(C)c2ccccc2C1CC(=O)c1ccc2ccccc2c1</smiles>

$0.6 \mathrm{mmol}$

\section{standard conditions}

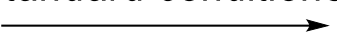<smiles>C[n+]1cc(C=O)c(/C=C(\O)c2ccc3ccccc3c2)c2ccccc21</smiles>

6

6'

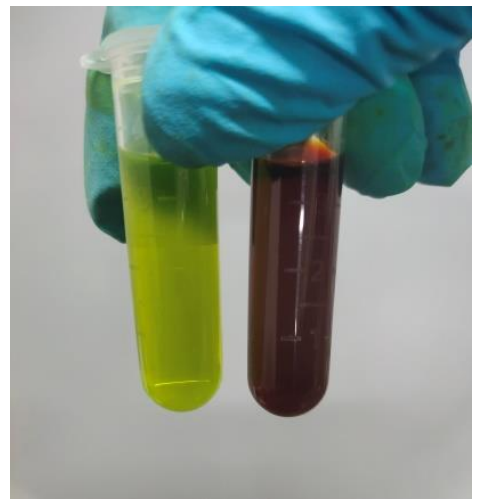

Figure S2. The fluorescence image of dihydroquinoline probe added after the completion of the reaction (left) and the standard condition with dihydroquinoline probe added (right). 
Spectrum from 13.wiff (sample 1) - Sample013, +TOF MS (100 - 1000) from $0.182 \mathrm{~min}$

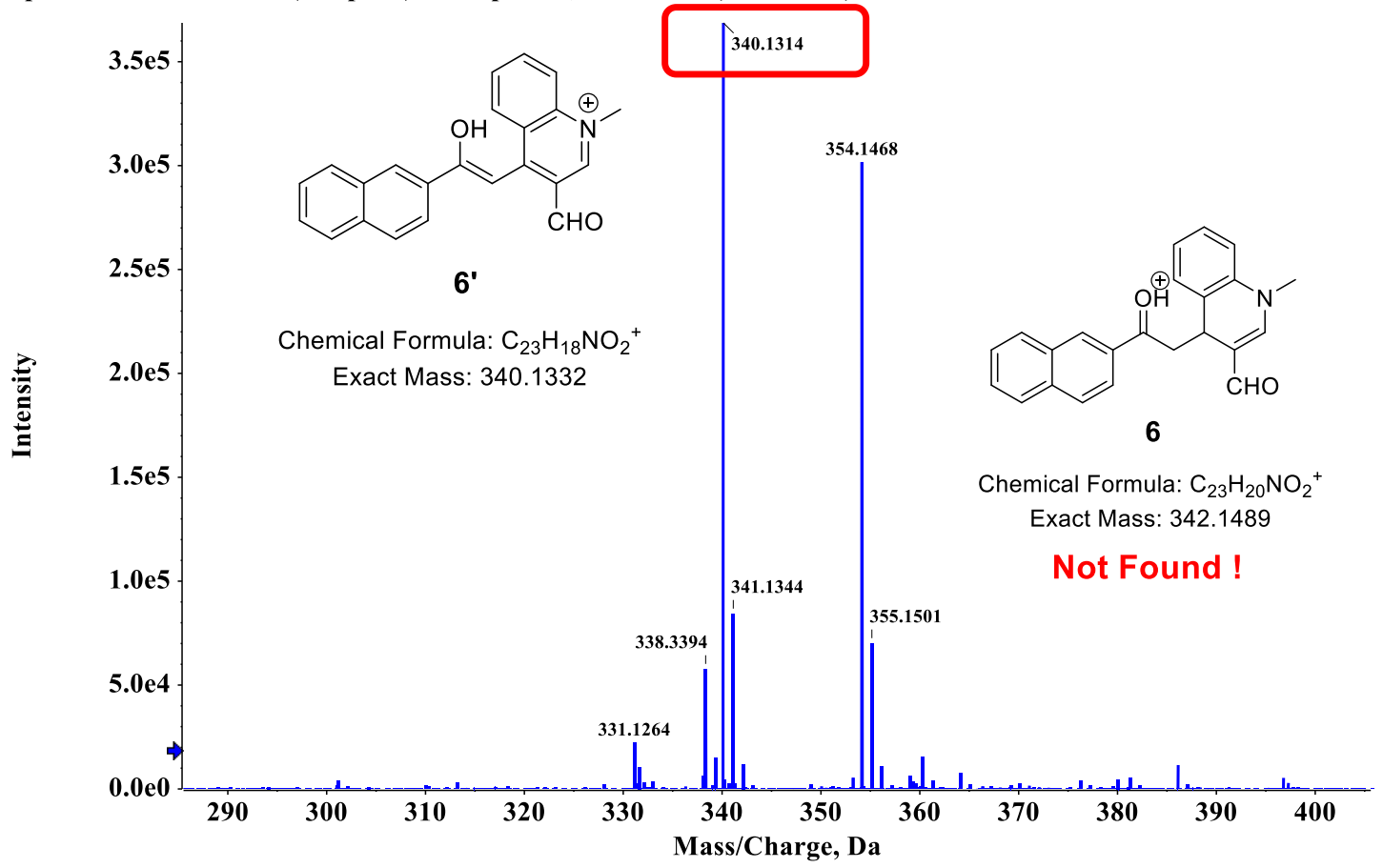




\section{Synthetic Application}

The reaction of was further scaled-up to $1 \mathrm{mmol}$ for $\mathbf{1 a}$. To a $25 \mathrm{~mL}$ vial was added $1 \mathbf{a}(1.0 \mathrm{mmol}, 507 \mathrm{mg})$, sulfonyl chloride $(2.5 \mathrm{mmol}, 2.5$ equiv. $), f a c-\operatorname{Ir}(\mathrm{ppy})_{3}(5.0 \mathrm{~mol} \%)$ and potassium phosphate $(2.0 \mathrm{mmol}, 2$ equiv.). The reaction flask was sealed, evacuated and back-filled with argon for three times. Then $10 \mathrm{~mL}$ degassed solvent $\left(\mathrm{CH}_{2} \mathrm{Cl}_{2} /\right.$ water $)$ $(v: v=10: 1)$ was injected into the sealed vial. The reaction mixture was stirred under the irradiation of $15 \mathrm{~W}$ blue LEDs $(450-455 \mathrm{~nm})$ at room temperature for 18 hours. Upon completion of the reaction, the solvent was filtered before evaporation under reduced pressure and the residue was recrystallized using petroleum ether/ethylacetate (3:1) to afford the target product (3) in 65\% yield (442 $\mathrm{mg})$.
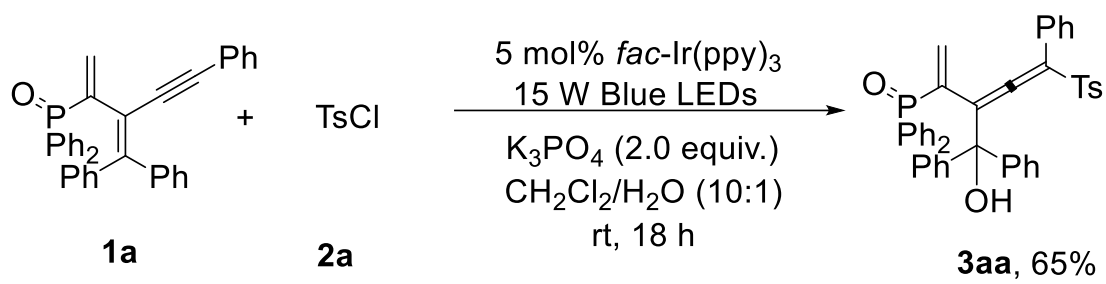

Synthetic application of the product 3aa to [3]dendralenes was then explored. To a $10 \mathrm{~mL}$ flask equipped with 3aa $(0.2 \mathrm{mmol}), \mathrm{SOCl}_{2}$ or $\mathrm{PBr}_{3}(0.6 \mathrm{mmol})$ and $2 \mathrm{~mL}$ anhydrous 1,2-dichloroethane. The reaction mixture was then heated to $50{ }^{\circ} \mathrm{C}$ in an oil bath for 6 hours under argon atmosphere. After all of the volatiles were removed under vacuum, the crude product was purified on flash chromatography (eluent: 1:2 (v/v) of ethyl acetate/petroleum ether) to afford [3]dendralenes (5a or $\mathbf{5 b}$ ), and the compounds were characterized by NMR and MS, see details as follows. 


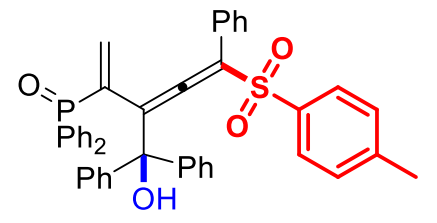

$3 a a$

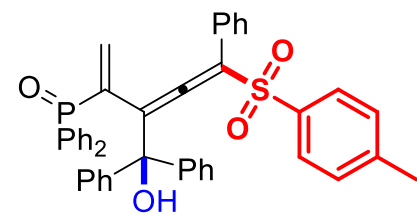

3aa<smiles>[3H]/C(=C(Cl)/C(C(=C)[PH](=O)c1ccccc1)=C(/c1ccccc1)[PH](=O)c1ccccc1)c1ccccc1</smiles>

$5 a, 65 \%$<smiles></smiles>

$5 b, 55 \%$ 


\section{X-Ray Crystallography Data of Compound 3aa}

Crystal 3aa (the ellipsoid contour percent probability level is 50\%) was obtained in solvent $\mathrm{CH}_{2} \mathrm{Cl}_{2} / n$-hexane $=1: 5$ through natural volatilization at room temperature.

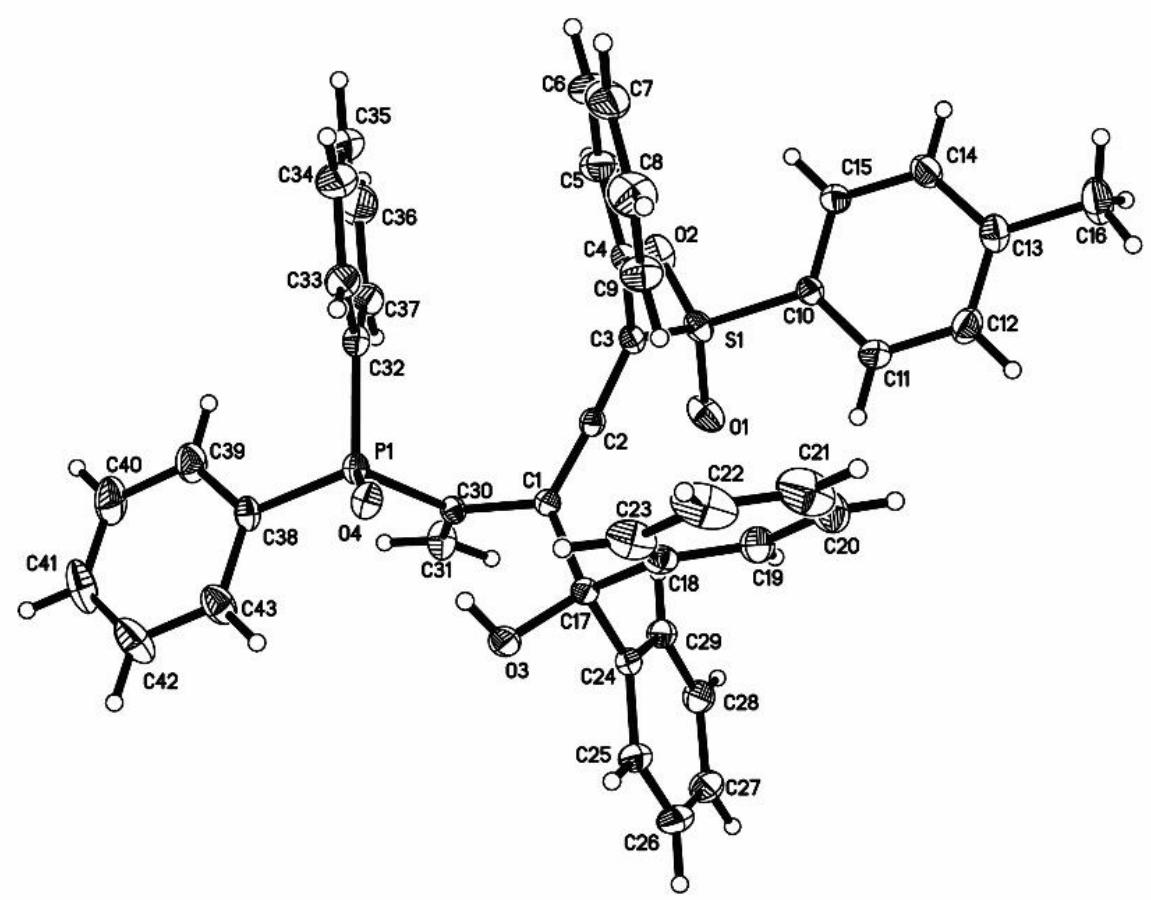

Table S5. Crystal data and structure refinement for 3aa.

$\begin{array}{lll}\text { Empirical formula } & \mathrm{C}_{43} \mathrm{H}_{35} \mathrm{O}_{4} \mathrm{PS} & \\ \text { Formula weight } & 678.74 & \\ \text { Temperature } & 296(2) \mathrm{K} & \\ \text { Wavelength } & 0.71073 \AA & \\ \text { Crystal system } & \text { Monoclinic } & \\ \text { Space group } & \mathrm{P} 2{ }_{1} & \alpha=90^{\circ} . \\ \text { Unit cell dimensions } & \mathrm{a}=10.5277(16) \AA & \beta=113.336(4)^{\circ} . \\ & \mathrm{b}=14.804(2) \AA & \gamma=90^{\circ} . \\ & \mathrm{c}=12.4712(18) \AA & \\ \text { Volume } & 1784.7(4) \AA^{3} & \\ \text { Z } & 2 & \\ \text { Density (calculated) } & 1.263 \mathrm{Mg}^{\circ} \mathrm{m}^{3} & \\ \text { Absorption coefficient } & 0.178 \mathrm{~mm}^{-1} & \\ \text { F(000) } & 712 & \\ \text { Crystal size } & 0.280 \times 0.260 \times 0.220 \mathrm{~mm}^{3}\end{array}$


Theta range for data collection

Index ranges

Reflections collected

Independent reflections

Completeness to theta $=25.242^{\circ}$

Absorption correction

Max. and min. transmission

Refinement method

Data / restraints / parameters

Goodness-of-fit on $\mathrm{F}^{2}$

Final $\mathrm{R}$ indices [I $>2 \operatorname{sigma}(\mathrm{I})]$

$\mathrm{R}$ indices (all data)

Absolute structure parameter

Extinction coefficient

Largest diff. peak and hole
2.107 to $27.588^{\circ}$.

$-13<=\mathrm{h}<=13,-19<=\mathrm{k}<=19,-15<=\mathrm{l}<=16$

28625

$8068[\mathrm{R}(\mathrm{int})=0.0280]$

$99.6 \%$

Semi-empirical from equivalents

0.7456 and 0.6936

Full-matrix least-squares on $\mathrm{F}^{2}$

8068 / 1 / 444

1.018

$\mathrm{R} 1=0.0373, \mathrm{wR} 2=0.0888$

$\mathrm{R} 1=0.0469, \mathrm{wR} 2=0.0930$

$0.367(17)$

$\mathrm{n} / \mathrm{a}$

0.217 and -0.219 e. $\AA^{-3}$

Table S6. Bond lengths $[\AA]$ and angles $\left[{ }^{\circ}\right]$ for $3 a a$.

$\begin{array}{ll}\mathrm{C}(1)-\mathrm{C}(2) & 1.300(3) \\ \mathrm{C}(1)-\mathrm{C}(30) & 1.499(3) \\ \mathrm{C}(1)-\mathrm{C}(17) & 1.542(3) \\ \mathrm{C}(2)-\mathrm{C}(3) & 1.306(3) \\ \mathrm{C}(3)-\mathrm{C}(4) & 1.480(4) \\ \mathrm{C}(3)-\mathrm{S}(1) & 1.783(2) \\ \mathrm{C}(4)-\mathrm{C}(9) & 1.378(4) \\ \mathrm{C}(4)-\mathrm{C}(5) & 1.382(4) \\ \mathrm{C}(5)-\mathrm{C}(6) & 1.383(5) \\ \mathrm{C}(5)-\mathrm{H}(5) & 0.9300 \\ \mathrm{C}(6)-\mathrm{C}(7) & 1.363(7) \\ \mathrm{C}(6)-\mathrm{H}(6) & 0.9300 \\ \mathrm{C}(7)-\mathrm{C}(8) & 1.357(6) \\ \mathrm{C}(7)-\mathrm{H}(7) & 0.9300 \\ \mathrm{C}(8)-\mathrm{C}(9) & 1.377(5) \\ \mathrm{C}(8)-\mathrm{H}(8) & 0.9300 \\ \mathrm{C}(9)-\mathrm{H}(9) & 0.9300 \\ \mathrm{C}(10)-\mathrm{C}(11) & 1.378(4) \\ \mathrm{C}(10)-\mathrm{C}(15) & 1.387(4) \\ \mathrm{C}(10)-\mathrm{S}(1) & 1.759(3) \\ & \\ & \end{array}$




\begin{tabular}{|c|c|}
\hline $\mathrm{C}(11)-\mathrm{C}(12)$ & $1.372(4)$ \\
\hline $\mathrm{C}(11)-\mathrm{H}(11)$ & 0.9300 \\
\hline $\mathrm{C}(12)-\mathrm{C}(13)$ & $1.379(5)$ \\
\hline $\mathrm{C}(12)-\mathrm{H}(12)$ & 0.9300 \\
\hline C(13)-C(14) & $1.381(4)$ \\
\hline$C(13)-C(16)$ & $1.504(4)$ \\
\hline $\mathrm{C}(14)-\mathrm{C}(15)$ & $1.373(4)$ \\
\hline $\mathrm{C}(14)-\mathrm{H}(14)$ & 0.9300 \\
\hline $\mathrm{C}(15)-\mathrm{H}(15)$ & 0.9300 \\
\hline $\mathrm{C}(16)-\mathrm{H}(16 \mathrm{~A})$ & 0.9600 \\
\hline $\mathrm{C}(16)-\mathrm{H}(16 \mathrm{~B})$ & 0.9600 \\
\hline $\mathrm{C}(16)-\mathrm{H}(16 \mathrm{C})$ & 0.9600 \\
\hline $\mathrm{C}(17)-\mathrm{O}(3)$ & $1.421(3)$ \\
\hline$C(17)-C(24)$ & $1.522(3)$ \\
\hline $\mathrm{C}(17)-\mathrm{C}(18)$ & $1.533(4)$ \\
\hline $\mathrm{C}(18)-\mathrm{C}(23)$ & $1.374(4)$ \\
\hline C(18)-C(19) & $1.379(4)$ \\
\hline C(19)-C(20) & $1.382(5)$ \\
\hline C(19)-H(19) & 0.9300 \\
\hline$C(20)-C(21)$ & $1.364(8)$ \\
\hline $\mathrm{C}(20)-\mathrm{H}(20)$ & 0.9300 \\
\hline $\mathrm{C}(21)-\mathrm{C}(22)$ & $1.365(8)$ \\
\hline $\mathrm{C}(21)-\mathrm{H}(21)$ & 0.9300 \\
\hline$C(22)-C(23)$ & $1.393(6)$ \\
\hline $\mathrm{C}(22)-\mathrm{H}(22)$ & 0.9300 \\
\hline $\mathrm{C}(23)-\mathrm{H}(23)$ & 0.9300 \\
\hline$C(24)-C(29)$ & $1.383(4)$ \\
\hline$C(24)-C(25)$ & $1.390(4)$ \\
\hline $\mathrm{C}(25)-\mathrm{C}(26)$ & $1.386(4)$ \\
\hline $\mathrm{C}(25)-\mathrm{H}(25)$ & 0.9300 \\
\hline$C(26)-C(27)$ & $1.358(5)$ \\
\hline $\mathrm{C}(26)-\mathrm{H}(26)$ & 0.9300 \\
\hline $\mathrm{C}(27)-\mathrm{C}(28)$ & $1.360(4)$ \\
\hline $\mathrm{C}(27)-\mathrm{H}(27)$ & 0.9300 \\
\hline C(28)-C(29) & $1.389(4)$ \\
\hline $\mathrm{C}(28)-\mathrm{H}(28)$ & 0.9300 \\
\hline $\mathrm{C}(29)-\mathrm{H}(29)$ & 0.9300 \\
\hline $\mathrm{C}(30)-\mathrm{C}(31)$ & $1.315(4)$ \\
\hline
\end{tabular}




\begin{tabular}{|c|c|}
\hline $\mathrm{C}(30)-\mathrm{P}(1)$ & $1.807(2)$ \\
\hline $\mathrm{C}(31)-\mathrm{H}(31 \mathrm{~A})$ & 0.9300 \\
\hline $\mathrm{C}(31)-\mathrm{H}(31 \mathrm{~B})$ & 0.9300 \\
\hline $\mathrm{C}(32)-\mathrm{C}(37)$ & $1.373(4)$ \\
\hline $\mathrm{C}(32)-\mathrm{C}(33)$ & $1.384(4)$ \\
\hline $\mathrm{C}(32)-\mathrm{P}(1)$ & $1.792(3)$ \\
\hline$C(33)-C(34)$ & $1.380(5)$ \\
\hline $\mathrm{C}(33)-\mathrm{H}(33)$ & 0.9300 \\
\hline $\mathrm{C}(34)-\mathrm{C}(35)$ & $1.359(7)$ \\
\hline C(34)-H(34) & 0.9300 \\
\hline$C(35)-C(36)$ & $1.355(7)$ \\
\hline $\mathrm{C}(35)-\mathrm{H}(35)$ & 0.9300 \\
\hline $\mathrm{C}(36)-\mathrm{C}(37)$ & $1.392(6)$ \\
\hline $\mathrm{C}(36)-\mathrm{H}(36)$ & 0.9300 \\
\hline C(37)-H(37) & 0.9300 \\
\hline $\mathrm{C}(38)-\mathrm{C}(39)$ & $1.378(4)$ \\
\hline $\mathrm{C}(38)-\mathrm{C}(43)$ & $1.379(4)$ \\
\hline $\mathrm{C}(38)-\mathrm{P}(1)$ & $1.799(3)$ \\
\hline$C(39)-C(40)$ & $1.382(5)$ \\
\hline C(39)-H(39) & 0.9300 \\
\hline $\mathrm{C}(40)-\mathrm{C}(41)$ & $1.346(6)$ \\
\hline $\mathrm{C}(40)-\mathrm{H}(40)$ & 0.9300 \\
\hline $\mathrm{C}(41)-\mathrm{C}(42)$ & $1.368(6)$ \\
\hline $\mathrm{C}(41)-\mathrm{H}(41)$ & 0.9300 \\
\hline $\mathrm{C}(42)-\mathrm{C}(43)$ & $1.377(5)$ \\
\hline $\mathrm{C}(42)-\mathrm{H}(42)$ & 0.9300 \\
\hline $\mathrm{C}(43)-\mathrm{H}(43)$ & 0.9300 \\
\hline $\mathrm{O}(1)-\mathrm{S}(1)$ & $1.428(2)$ \\
\hline $\mathrm{O}(2)-\mathrm{S}(1)$ & $1.432(2)$ \\
\hline $\mathrm{O}(3)-\mathrm{H}(3)$ & 0.8200 \\
\hline $\mathrm{O}(4)-\mathrm{P}(1)$ & $1.4903(18)$ \\
\hline $\mathrm{C}(2)-\mathrm{C}(1)-\mathrm{C}(30)$ & $118.7(2)$ \\
\hline $\mathrm{C}(2)-\mathrm{C}(1)-\mathrm{C}(17)$ & $123.5(2)$ \\
\hline $\mathrm{C}(30)-\mathrm{C}(1)-\mathrm{C}(17)$ & 117.66(19) \\
\hline $\mathrm{C}(1)-\mathrm{C}(2)-\mathrm{C}(3)$ & $176.3(2)$ \\
\hline $\mathrm{C}(2)-\mathrm{C}(3)-\mathrm{C}(4)$ & $124.4(2)$ \\
\hline $\mathrm{C}(2)-\mathrm{C}(3)-\mathrm{S}(1)$ & 115.81(19) \\
\hline
\end{tabular}




\begin{tabular}{|c|c|}
\hline $\mathrm{C}(4)-\mathrm{C}(3)-\mathrm{S}(1)$ & $119.83(17)$ \\
\hline$C(9)-C(4)-C(5)$ & 118.7(3) \\
\hline $\mathrm{C}(9)-\mathrm{C}(4)-\mathrm{C}(3)$ & $118.4(2)$ \\
\hline$C(5)-C(4)-C(3)$ & $122.9(3)$ \\
\hline$C(4)-C(5)-C(6)$ & 119.3(4) \\
\hline $\mathrm{C}(4)-\mathrm{C}(5)-\mathrm{H}(5)$ & 120.3 \\
\hline $\mathrm{C}(6)-\mathrm{C}(5)-\mathrm{H}(5)$ & 120.3 \\
\hline$C(7)-C(6)-C(5)$ & $121.4(4)$ \\
\hline $\mathrm{C}(7)-\mathrm{C}(6)-\mathrm{H}(6)$ & 119.3 \\
\hline $\mathrm{C}(5)-\mathrm{C}(6)-\mathrm{H}(6)$ & 119.3 \\
\hline$C(8)-C(7)-C(6)$ & $119.3(4)$ \\
\hline $\mathrm{C}(8)-\mathrm{C}(7)-\mathrm{H}(7)$ & 120.3 \\
\hline $\mathrm{C}(6)-\mathrm{C}(7)-\mathrm{H}(7)$ & 120.3 \\
\hline $\mathrm{C}(7)-\mathrm{C}(8)-\mathrm{C}(9)$ & $120.4(4)$ \\
\hline $\mathrm{C}(7)-\mathrm{C}(8)-\mathrm{H}(8)$ & 119.8 \\
\hline $\mathrm{C}(9)-\mathrm{C}(8)-\mathrm{H}(8)$ & 119.8 \\
\hline $\mathrm{C}(8)-\mathrm{C}(9)-\mathrm{C}(4)$ & $120.8(3)$ \\
\hline $\mathrm{C}(8)-\mathrm{C}(9)-\mathrm{H}(9)$ & 119.6 \\
\hline $\mathrm{C}(4)-\mathrm{C}(9)-\mathrm{H}(9)$ & 119.6 \\
\hline $\mathrm{C}(11)-\mathrm{C}(10)-\mathrm{C}(15)$ & $120.0(2)$ \\
\hline $\mathrm{C}(11)-\mathrm{C}(10)-\mathrm{S}(1)$ & $120.5(2)$ \\
\hline$C(15)-C(10)-S(1)$ & $119.5(2)$ \\
\hline $\mathrm{C}(12)-\mathrm{C}(11)-\mathrm{C}(10)$ & 119.6(3) \\
\hline $\mathrm{C}(12)-\mathrm{C}(11)-\mathrm{H}(11)$ & 120.2 \\
\hline $\mathrm{C}(10)-\mathrm{C}(11)-\mathrm{H}(11)$ & 120.2 \\
\hline $\mathrm{C}(11)-\mathrm{C}(12)-\mathrm{C}(13)$ & $121.6(3)$ \\
\hline $\mathrm{C}(11)-\mathrm{C}(12)-\mathrm{H}(12)$ & 119.2 \\
\hline $\mathrm{C}(13)-\mathrm{C}(12)-\mathrm{H}(12)$ & 119.2 \\
\hline $\mathrm{C}(12)-\mathrm{C}(13)-\mathrm{C}(14)$ & $118.0(3)$ \\
\hline $\mathrm{C}(12)-\mathrm{C}(13)-\mathrm{C}(16)$ & $121.5(3)$ \\
\hline $\mathrm{C}(14)-\mathrm{C}(13)-\mathrm{C}(16)$ & $120.6(3)$ \\
\hline $\mathrm{C}(15)-\mathrm{C}(14)-\mathrm{C}(13)$ & $121.6(3)$ \\
\hline $\mathrm{C}(15)-\mathrm{C}(14)-\mathrm{H}(14)$ & 119.2 \\
\hline $\mathrm{C}(13)-\mathrm{C}(14)-\mathrm{H}(14)$ & 119.2 \\
\hline $\mathrm{C}(14)-\mathrm{C}(15)-\mathrm{C}(10)$ & 119.3(3) \\
\hline $\mathrm{C}(14)-\mathrm{C}(15)-\mathrm{H}(15)$ & 120.4 \\
\hline $\mathrm{C}(10)-\mathrm{C}(15)-\mathrm{H}(15)$ & 120.4 \\
\hline $\mathrm{C}(13)-\mathrm{C}(16)-\mathrm{H}(16 \mathrm{~A})$ & 109.5 \\
\hline
\end{tabular}




\begin{tabular}{|c|c|}
\hline $\mathrm{C}(13)-\mathrm{C}(16)-\mathrm{H}(16 \mathrm{~B})$ & 109.5 \\
\hline $\mathrm{H}(16 \mathrm{~A})-\mathrm{C}(16)-\mathrm{H}(16 \mathrm{~B})$ & 109.5 \\
\hline $\mathrm{C}(13)-\mathrm{C}(16)-\mathrm{H}(16 \mathrm{C})$ & 109.5 \\
\hline$H(16 A)-C(16)-H(16 C)$ & 109.5 \\
\hline $\mathrm{H}(16 \mathrm{~B})-\mathrm{C}(16)-\mathrm{H}(16 \mathrm{C})$ & 109.5 \\
\hline $\mathrm{O}(3)-\mathrm{C}(17)-\mathrm{C}(24)$ & $105.82(19)$ \\
\hline $\mathrm{O}(3)-\mathrm{C}(17)-\mathrm{C}(18)$ & $110.2(2)$ \\
\hline $\mathrm{C}(24)-\mathrm{C}(17)-\mathrm{C}(18)$ & $111.0(2)$ \\
\hline $\mathrm{O}(3)-\mathrm{C}(17)-\mathrm{C}(1)$ & $109.24(19)$ \\
\hline $\mathrm{C}(24)-\mathrm{C}(17)-\mathrm{C}(1)$ & $110.9(2)$ \\
\hline $\mathrm{C}(18)-\mathrm{C}(17)-\mathrm{C}(1)$ & $109.5(2)$ \\
\hline $\mathrm{C}(23)-\mathrm{C}(18)-\mathrm{C}(19)$ & $118.2(3)$ \\
\hline $\mathrm{C}(23)-\mathrm{C}(18)-\mathrm{C}(17)$ & $120.4(3)$ \\
\hline C(19)-C(18)-C(17) & 121.3(3) \\
\hline $\mathrm{C}(18)-\mathrm{C}(19)-\mathrm{C}(20)$ & $121.2(4)$ \\
\hline $\mathrm{C}(18)-\mathrm{C}(19)-\mathrm{H}(19)$ & 119.4 \\
\hline $\mathrm{C}(20)-\mathrm{C}(19)-\mathrm{H}(19)$ & 119.4 \\
\hline $\mathrm{C}(21)-\mathrm{C}(20)-\mathrm{C}(19)$ & $120.0(5)$ \\
\hline $\mathrm{C}(21)-\mathrm{C}(20)-\mathrm{H}(20)$ & 120.0 \\
\hline $\mathrm{C}(19)-\mathrm{C}(20)-\mathrm{H}(20)$ & 120.0 \\
\hline $\mathrm{C}(20)-\mathrm{C}(21)-\mathrm{C}(22)$ & $119.8(4)$ \\
\hline $\mathrm{C}(20)-\mathrm{C}(21)-\mathrm{H}(21)$ & 120.1 \\
\hline $\mathrm{C}(22)-\mathrm{C}(21)-\mathrm{H}(21)$ & 120.1 \\
\hline$C(21)-C(22)-C(23)$ & $120.2(5)$ \\
\hline $\mathrm{C}(21)-\mathrm{C}(22)-\mathrm{H}(22)$ & 119.9 \\
\hline $\mathrm{C}(23)-\mathrm{C}(22)-\mathrm{H}(22)$ & 119.9 \\
\hline $\mathrm{C}(18)-\mathrm{C}(23)-\mathrm{C}(22)$ & $120.6(4)$ \\
\hline $\mathrm{C}(18)-\mathrm{C}(23)-\mathrm{H}(23)$ & 119.7 \\
\hline $\mathrm{C}(22)-\mathrm{C}(23)-\mathrm{H}(23)$ & 119.7 \\
\hline $\mathrm{C}(29)-\mathrm{C}(24)-\mathrm{C}(25)$ & $118.7(2)$ \\
\hline $\mathrm{C}(29)-\mathrm{C}(24)-\mathrm{C}(17)$ & $122.9(2)$ \\
\hline $\mathrm{C}(25)-\mathrm{C}(24)-\mathrm{C}(17)$ & $118.4(2)$ \\
\hline$C(26)-C(25)-C(24)$ & 119.6(3) \\
\hline $\mathrm{C}(26)-\mathrm{C}(25)-\mathrm{H}(25)$ & 120.2 \\
\hline $\mathrm{C}(24)-\mathrm{C}(25)-\mathrm{H}(25)$ & 120.2 \\
\hline$C(27)-C(26)-C(25)$ & $120.9(3)$ \\
\hline $\mathrm{C}(27)-\mathrm{C}(26)-\mathrm{H}(26)$ & 119.5 \\
\hline $\mathrm{C}(25)-\mathrm{C}(26)-\mathrm{H}(26)$ & 119.5 \\
\hline
\end{tabular}




\begin{tabular}{|c|c|}
\hline $\mathrm{C}(26)-\mathrm{C}(27)-\mathrm{C}(28)$ & $120.2(3)$ \\
\hline $\mathrm{C}(26)-\mathrm{C}(27)-\mathrm{H}(27)$ & 119.9 \\
\hline $\mathrm{C}(28)-\mathrm{C}(27)-\mathrm{H}(27)$ & 119.9 \\
\hline $\mathrm{C}(27)-\mathrm{C}(28)-\mathrm{C}(29)$ & $120.1(3)$ \\
\hline $\mathrm{C}(27)-\mathrm{C}(28)-\mathrm{H}(28)$ & 119.9 \\
\hline $\mathrm{C}(29)-\mathrm{C}(28)-\mathrm{H}(28)$ & 119.9 \\
\hline $\mathrm{C}(24)-\mathrm{C}(29)-\mathrm{C}(28)$ & $120.4(3)$ \\
\hline $\mathrm{C}(24)-\mathrm{C}(29)-\mathrm{H}(29)$ & 119.8 \\
\hline $\mathrm{C}(28)-\mathrm{C}(29)-\mathrm{H}(29)$ & 119.8 \\
\hline $\mathrm{C}(31)-\mathrm{C}(30)-\mathrm{C}(1)$ & $124.3(2)$ \\
\hline $\mathrm{C}(31)-\mathrm{C}(30)-\mathrm{P}(1)$ & $123.6(2)$ \\
\hline $\mathrm{C}(1)-\mathrm{C}(30)-\mathrm{P}(1)$ & $112.14(16)$ \\
\hline $\mathrm{C}(30)-\mathrm{C}(31)-\mathrm{H}(31 \mathrm{~A})$ & 120.0 \\
\hline $\mathrm{C}(30)-\mathrm{C}(31)-\mathrm{H}(31 \mathrm{~B})$ & 120.0 \\
\hline $\mathrm{H}(31 \mathrm{~A})-\mathrm{C}(31)-\mathrm{H}(31 \mathrm{~B})$ & 120.0 \\
\hline $\mathrm{C}(37)-\mathrm{C}(32)-\mathrm{C}(33)$ & 119.2(3) \\
\hline $\mathrm{C}(37)-\mathrm{C}(32)-\mathrm{P}(1)$ & $123.2(2)$ \\
\hline $\mathrm{C}(33)-\mathrm{C}(32)-\mathrm{P}(1)$ & $117.5(2)$ \\
\hline $\mathrm{C}(34)-\mathrm{C}(33)-\mathrm{C}(32)$ & $120.4(4)$ \\
\hline $\mathrm{C}(34)-\mathrm{C}(33)-\mathrm{H}(33)$ & 119.8 \\
\hline $\mathrm{C}(32)-\mathrm{C}(33)-\mathrm{H}(33)$ & 119.8 \\
\hline $\mathrm{C}(35)-\mathrm{C}(34)-\mathrm{C}(33)$ & $119.6(4)$ \\
\hline $\mathrm{C}(35)-\mathrm{C}(34)-\mathrm{H}(34)$ & 120.2 \\
\hline $\mathrm{C}(33)-\mathrm{C}(34)-\mathrm{H}(34)$ & 120.2 \\
\hline$C(36)-C(35)-C(34)$ & $121.0(4)$ \\
\hline $\mathrm{C}(36)-\mathrm{C}(35)-\mathrm{H}(35)$ & 119.5 \\
\hline $\mathrm{C}(34)-\mathrm{C}(35)-\mathrm{H}(35)$ & 119.5 \\
\hline $\mathrm{C}(35)-\mathrm{C}(36)-\mathrm{C}(37)$ & $120.1(4)$ \\
\hline $\mathrm{C}(35)-\mathrm{C}(36)-\mathrm{H}(36)$ & 119.9 \\
\hline $\mathrm{C}(37)-\mathrm{C}(36)-\mathrm{H}(36)$ & 119.9 \\
\hline $\mathrm{C}(32)-\mathrm{C}(37)-\mathrm{C}(36)$ & 119.7(4) \\
\hline $\mathrm{C}(32)-\mathrm{C}(37)-\mathrm{H}(37)$ & 120.2 \\
\hline $\mathrm{C}(36)-\mathrm{C}(37)-\mathrm{H}(37)$ & 120.2 \\
\hline $\mathrm{C}(39)-\mathrm{C}(38)-\mathrm{C}(43)$ & $118.6(3)$ \\
\hline $\mathrm{C}(39)-\mathrm{C}(38)-\mathrm{P}(1)$ & $124.4(2)$ \\
\hline $\mathrm{C}(43)-\mathrm{C}(38)-\mathrm{P}(1)$ & $116.9(2)$ \\
\hline $\mathrm{C}(38)-\mathrm{C}(39)-\mathrm{C}(40)$ & $120.5(3)$ \\
\hline $\mathrm{C}(38)-\mathrm{C}(39)-\mathrm{H}(39)$ & 119.8 \\
\hline
\end{tabular}




\begin{tabular}{|c|c|}
\hline $\mathrm{C}(40)-\mathrm{C}(39)-\mathrm{H}(39)$ & 119.8 \\
\hline $\mathrm{C}(41)-\mathrm{C}(40)-\mathrm{C}(39)$ & $120.1(3)$ \\
\hline $\mathrm{C}(41)-\mathrm{C}(40)-\mathrm{H}(40)$ & 119.9 \\
\hline $\mathrm{C}(39)-\mathrm{C}(40)-\mathrm{H}(40)$ & 119.9 \\
\hline$C(40)-C(41)-C(42)$ & $120.5(3)$ \\
\hline $\mathrm{C}(40)-\mathrm{C}(41)-\mathrm{H}(41)$ & 119.8 \\
\hline $\mathrm{C}(42)-\mathrm{C}(41)-\mathrm{H}(41)$ & 119.8 \\
\hline$C(41)-C(42)-C(43)$ & $119.9(4)$ \\
\hline $\mathrm{C}(41)-\mathrm{C}(42)-\mathrm{H}(42)$ & 120.0 \\
\hline $\mathrm{C}(43)-\mathrm{C}(42)-\mathrm{H}(42)$ & 120.0 \\
\hline $\mathrm{C}(42)-\mathrm{C}(43)-\mathrm{C}(38)$ & $120.3(3)$ \\
\hline $\mathrm{C}(42)-\mathrm{C}(43)-\mathrm{H}(43)$ & 119.8 \\
\hline $\mathrm{C}(38)-\mathrm{C}(43)-\mathrm{H}(43)$ & 119.8 \\
\hline $\mathrm{C}(17)-\mathrm{O}(3)-\mathrm{H}(3)$ & 109.5 \\
\hline $\mathrm{O}(4)-\mathrm{P}(1)-\mathrm{C}(32)$ & $112.53(12)$ \\
\hline $\mathrm{O}(4)-\mathrm{P}(1)-\mathrm{C}(38)$ & $111.06(12)$ \\
\hline $\mathrm{C}(32)-\mathrm{P}(1)-\mathrm{C}(38)$ & $108.48(13)$ \\
\hline $\mathrm{O}(4)-\mathrm{P}(1)-\mathrm{C}(30)$ & $110.39(11)$ \\
\hline $\mathrm{C}(32)-\mathrm{P}(1)-\mathrm{C}(30)$ & $105.66(11)$ \\
\hline $\mathrm{C}(38)-\mathrm{P}(1)-\mathrm{C}(30)$ & $108.50(12)$ \\
\hline $\mathrm{O}(1)-\mathrm{S}(1)-\mathrm{O}(2)$ & $119.05(15)$ \\
\hline $\mathrm{O}(1)-\mathrm{S}(1)-\mathrm{C}(10)$ & $108.05(13)$ \\
\hline $\mathrm{O}(2)-\mathrm{S}(1)-\mathrm{C}(10)$ & $107.52(12)$ \\
\hline $\mathrm{O}(1)-\mathrm{S}(1)-\mathrm{C}(3)$ & $107.57(12)$ \\
\hline $\mathrm{O}(2)-\mathrm{S}(1)-\mathrm{C}(3)$ & $107.89(13)$ \\
\hline$C(10)-S(1)-C(3)$ & $106.07(11)$ \\
\hline
\end{tabular}

Symmetry transformations used to generate equivalent atoms:

Table S7. Hydrogen bonds [ $\left[\AA\right.$ and $\left.{ }^{\circ}\right]$ for $3 a$.

\begin{tabular}{lcccc}
\hline D-H...A & $d(D-H)$ & $d(H \ldots A)$ & $d(D \ldots A)$ & $<($ DHA $)$ \\
\hline $\mathrm{O}(3)-\mathrm{H}(3) \ldots \mathrm{O}(4)$ & 0.82 & 1.90 & $2.694(3)$ & 161.6 \\
\hline
\end{tabular}

Symmetry transformations used to generate equivalent atoms: 


\section{0. 'X-Ray Crystallography Data of Compound 4}

Crystal 4 (the ellipsoid contour percent probability level is 50\%) was obtained in solvent $\mathrm{CH}_{2} \mathrm{Cl}_{2} / n$-hexane $=1: 5$ through natural volatilization at room temperature.

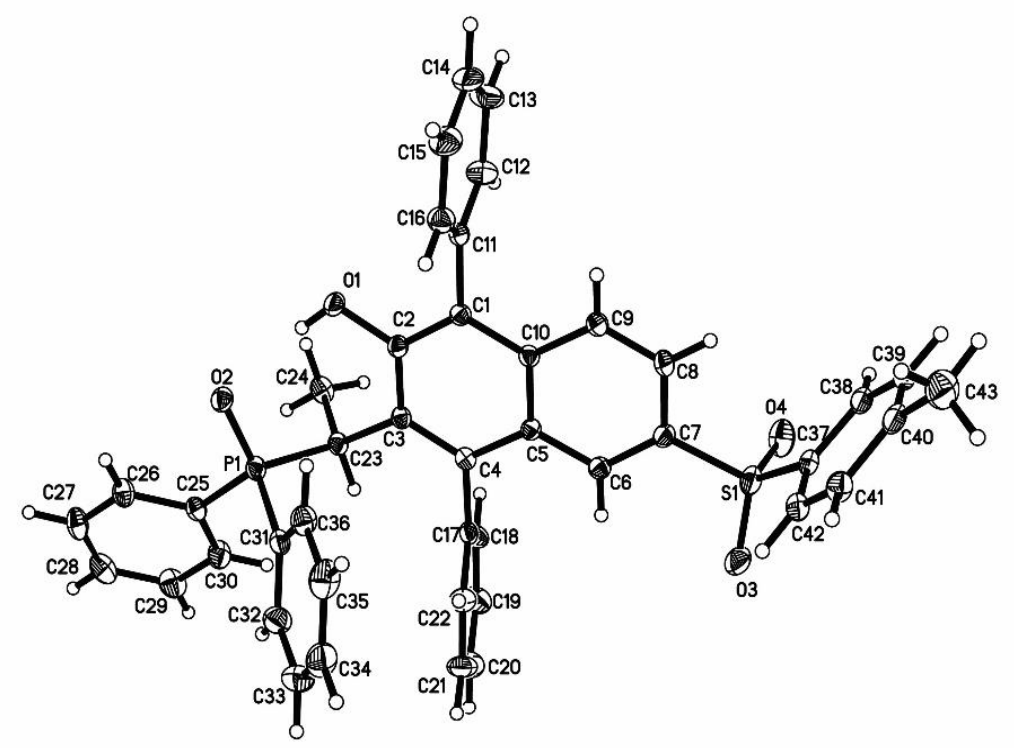

Table S8. Crystal data and structure refinement for 4 .

Identification code

Empirical formula

Formula weight

Temperature

Wavelength

Crystal system

Space group

Unit cell dimensions

Volume

$\mathrm{Z}$

Density (calculated)

Absorption coefficient

$\mathrm{F}(000)$

Crystal size

Theta range for data collection

Index ranges
ZCY200918_1_a

C43 H35 O4 P S

678.74

296(2) K

$0.71073 \AA$

Monoclinic

$\mathrm{P} 21 / \mathrm{n}$

$\mathrm{a}=16.901(3) \AA$

$\alpha=90^{\circ}$.

$\mathrm{b}=12.234(3) \AA$

$\beta=103.849(6)^{\circ}$.

$\mathrm{c}=17.112(3) \AA$

$\gamma=90^{\circ}$.

3435.4(12) $\AA^{3}$

4

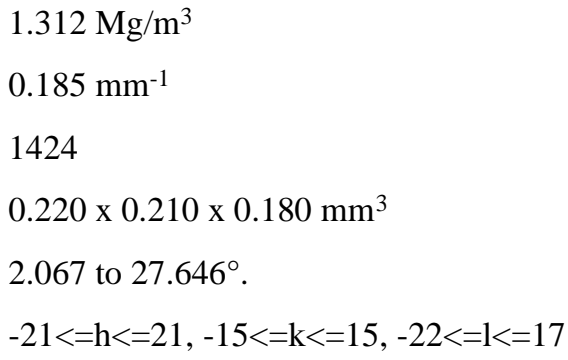

$1.312 \mathrm{Mg} / \mathrm{m}^{3}$

$0.185 \mathrm{~mm}^{-1}$

1424

$0.220 \times 0.210 \times 0.180 \mathrm{~mm}^{3}$

2.067 to $27.646^{\circ}$.

$-21<=\mathrm{h}<=21,-15<=\mathrm{k}<=15,-22<=1<=17$ 
Reflections collected

Independent reflections

Completeness to theta $=25.242^{\circ}$

Absorption correction

Max. and min. transmission

Refinement method

Data / restraints / parameters

Goodness-of-fit on $\mathrm{F}^{2}$

Final R indices [I $>2 \operatorname{sigma}(\mathrm{I})]$

$\mathrm{R}$ indices (all data)

Extinction coefficient

Largest diff. peak and hole
38457

7915 [R(int) $=0.0890]$

$99.5 \%$

Semi-empirical from equivalents

0.7456 and 0.6964

Full-matrix least-squares on $\mathrm{F}^{2}$

7915 / 15 / 444

1.008

$\mathrm{R} 1=0.0620, \mathrm{wR} 2=0.1142$

$\mathrm{R} 1=0.1606, \mathrm{wR} 2=0.1414$

$\mathrm{n} / \mathrm{a}$

0.284 and -0.307 e. $\AA^{-3}$

Table S9. Bond lengths $[\AA ̊]$ and angles $\left[^{\circ}\right]$ for 4 .

\begin{tabular}{ll}
\hline $\mathrm{C}(1)-\mathrm{C}(2)$ & $1.378(3)$ \\
$\mathrm{C}(1)-\mathrm{C}(10)$ & $1.414(3)$ \\
$\mathrm{C}(1)-\mathrm{C}(11)$ & $1.494(3)$ \\
$\mathrm{C}(2)-\mathrm{O}(1)$ & $1.358(3)$ \\
$\mathrm{C}(2)-\mathrm{C}(3)$ & $1.430(3)$ \\
$\mathrm{C}(3)-\mathrm{C}(4)$ & $1.382(3)$ \\
$\mathrm{C}(3)-\mathrm{C}(23)$ & $1.529(3)$ \\
$\mathrm{C}(4)-\mathrm{C}(5)$ & $1.425(3)$ \\
$\mathrm{C}(4)-\mathrm{C}(17)$ & $1.502(3)$ \\
$\mathrm{C}(5)-\mathrm{C}(6)$ & $1.412(3)$ \\
$\mathrm{C}(5)-\mathrm{C}(10)$ & $1.420(3)$ \\
$\mathrm{C}(6)-\mathrm{C}(7)$ & $1.367(3)$ \\
$\mathrm{C}(6)-\mathrm{H}(6)$ & 0.9300 \\
$\mathrm{C}(7)-\mathrm{C}(8)$ & $1.395(4)$ \\
$\mathrm{C}(7)-\mathrm{S}(1)$ & $1.769(3)$ \\
$\mathrm{C}(8)-\mathrm{C}(9)$ & $1.358(4)$ \\
$\mathrm{C}(8)-\mathrm{H}(8)$ & 0.9300 \\
$\mathrm{C}(9)-\mathrm{C}(10)$ & $1.415(3)$ \\
$\mathrm{C}(9)-\mathrm{H}(9)$ & 0.9300 \\
$\mathrm{C}(11)-\mathrm{C}(16)$ & $1.373(4)$ \\
$\mathrm{C}(11)-\mathrm{C}(12)$ & $1.373(4)$ \\
$\mathrm{C}(12)-\mathrm{C}(13)$ & $1.378(4)$ \\
$\mathrm{C}(12)-\mathrm{H}(12)$ & 0.9300 \\
&
\end{tabular}




\begin{tabular}{|c|c|}
\hline $\mathrm{C}(13)-\mathrm{C}(14)$ & $1.371(5)$ \\
\hline $\mathrm{C}(13)-\mathrm{H}(13)$ & 0.9300 \\
\hline $\mathrm{C}(14)-\mathrm{C}(15)$ & $1.365(5)$ \\
\hline $\mathrm{C}(14)-\mathrm{H}(14)$ & 0.9300 \\
\hline$C(15)-C(16)$ & $1.373(4)$ \\
\hline $\mathrm{C}(15)-\mathrm{H}(15)$ & 0.9300 \\
\hline $\mathrm{C}(16)-\mathrm{H}(16)$ & 0.9300 \\
\hline$C(17)-C(22)$ & $1.378(4)$ \\
\hline $\mathrm{C}(17)-\mathrm{C}(18)$ & $1.383(4)$ \\
\hline $\mathrm{C}(18)-\mathrm{C}(19)$ & $1.366(4)$ \\
\hline $\mathrm{C}(18)-\mathrm{H}(18)$ & 0.9300 \\
\hline C(19)-C(20) & $1.367(5)$ \\
\hline C(19)-H(19) & 0.9300 \\
\hline$C(20)-C(21)$ & $1.361(4)$ \\
\hline $\mathrm{C}(20)-\mathrm{H}(20)$ & 0.9300 \\
\hline $\mathrm{C}(21)-\mathrm{C}(22)$ & $1.380(4)$ \\
\hline $\mathrm{C}(21)-\mathrm{H}(21)$ & 0.9300 \\
\hline $\mathrm{C}(22)-\mathrm{H}(22)$ & 0.9300 \\
\hline $\mathrm{C}(23)-\mathrm{C}(24)$ & $1.536(3)$ \\
\hline $\mathrm{C}(23)-\mathrm{P}(1)$ & $1.807(3)$ \\
\hline $\mathrm{C}(23)-\mathrm{H}(23)$ & 0.9800 \\
\hline $\mathrm{C}(24)-\mathrm{H}(24 \mathrm{~A})$ & 0.9600 \\
\hline $\mathrm{C}(24)-\mathrm{H}(24 \mathrm{~B})$ & 0.9600 \\
\hline $\mathrm{C}(24)-\mathrm{H}(24 \mathrm{C})$ & 0.9600 \\
\hline$C(25)-C(26)$ & $1.377(4)$ \\
\hline$C(25)-C(30)$ & $1.386(4)$ \\
\hline $\mathrm{C}(25)-\mathrm{P}(1)$ & $1.802(2)$ \\
\hline $\mathrm{C}(26)-\mathrm{C}(27)$ & $1.387(4)$ \\
\hline $\mathrm{C}(26)-\mathrm{H}(26)$ & 0.9300 \\
\hline $\mathrm{C}(27)-\mathrm{C}(28)$ & $1.362(4)$ \\
\hline $\mathrm{C}(27)-\mathrm{H}(27)$ & 0.9300 \\
\hline $\mathrm{C}(28)-\mathrm{C}(29)$ & $1.366(4)$ \\
\hline $\mathrm{C}(28)-\mathrm{H}(28)$ & 0.9300 \\
\hline $\mathrm{C}(29)-\mathrm{C}(30)$ & $1.380(4)$ \\
\hline C(29)-H(29) & 0.9300 \\
\hline $\mathrm{C}(30)-\mathrm{H}(30)$ & 0.9300 \\
\hline $\mathrm{C}(31)-\mathrm{C}(32)$ & $1.377(4)$ \\
\hline C(31)-C(36) & $1.388(4)$ \\
\hline
\end{tabular}




\begin{tabular}{|c|c|}
\hline $\mathrm{C}(31)-\mathrm{P}(1)$ & $1.792(3)$ \\
\hline $\mathrm{C}(32)-\mathrm{C}(33)$ & $1.382(4)$ \\
\hline $\mathrm{C}(32)-\mathrm{H}(32)$ & 0.9300 \\
\hline $\mathrm{C}(33)-\mathrm{C}(34)$ & $1.361(5)$ \\
\hline $\mathrm{C}(33)-\mathrm{H}(33)$ & 0.9300 \\
\hline$C(34)-C(35)$ & $1.363(5)$ \\
\hline $\mathrm{C}(34)-\mathrm{H}(34)$ & 0.9300 \\
\hline$C(35)-C(36)$ & $1.378(4)$ \\
\hline $\mathrm{C}(35)-\mathrm{H}(35)$ & 0.9300 \\
\hline $\mathrm{C}(36)-\mathrm{H}(36)$ & 0.9300 \\
\hline $\mathrm{C}(37)-\mathrm{C}(38)$ & $1.380(4)$ \\
\hline $\mathrm{C}(37)-\mathrm{C}(42)$ & $1.381(4)$ \\
\hline $\mathrm{C}(37)-\mathrm{S}(1)$ & $1.759(3)$ \\
\hline $\mathrm{C}(38)-\mathrm{C}(39)$ & $1.368(4)$ \\
\hline $\mathrm{C}(38)-\mathrm{H}(38)$ & 0.9300 \\
\hline $\mathrm{C}(39)-\mathrm{C}(40)$ & $1.392(4)$ \\
\hline $\mathrm{C}(39)-\mathrm{H}(39)$ & 0.9300 \\
\hline$C(40)-C(41)$ & $1.396(4)$ \\
\hline $\mathrm{C}(40)-\mathrm{C}(43)$ & $1.514(4)$ \\
\hline $\mathrm{C}(41)-\mathrm{C}(42)$ & $1.363(4)$ \\
\hline $\mathrm{C}(41)-\mathrm{H}(41)$ & 0.9300 \\
\hline $\mathrm{C}(42)-\mathrm{H}(42)$ & 0.9300 \\
\hline $\mathrm{C}(43)-\mathrm{H}(43 \mathrm{~A})$ & 0.9600 \\
\hline $\mathrm{C}(43)-\mathrm{H}(43 \mathrm{~B})$ & 0.9600 \\
\hline $\mathrm{C}(43)-\mathrm{H}(43 \mathrm{C})$ & 0.9600 \\
\hline $\mathrm{O}(1)-\mathrm{H}(1)$ & 0.8200 \\
\hline $\mathrm{O}(2)-\mathrm{P}(1)$ & $1.4923(18)$ \\
\hline $\mathrm{O}(3)-\mathrm{S}(1)$ & $1.427(2)$ \\
\hline $\mathrm{O}(4)-\mathrm{S}(1)$ & $1.443(2)$ \\
\hline$C(2)-C(1)-C(10)$ & $119.6(2)$ \\
\hline$C(2)-C(1)-C(11)$ & $121.6(2)$ \\
\hline $\mathrm{C}(10)-\mathrm{C}(1)-\mathrm{C}(11)$ & $118.7(2)$ \\
\hline $\mathrm{O}(1)-\mathrm{C}(2)-\mathrm{C}(1)$ & $116.7(2)$ \\
\hline $\mathrm{O}(1)-\mathrm{C}(2)-\mathrm{C}(3)$ & $121.9(2)$ \\
\hline $\mathrm{C}(1)-\mathrm{C}(2)-\mathrm{C}(3)$ & $121.4(2)$ \\
\hline $\mathrm{C}(4)-\mathrm{C}(3)-\mathrm{C}(2)$ & $119.0(2)$ \\
\hline$C(4)-C(3)-C(23)$ & $120.0(2)$ \\
\hline
\end{tabular}




\begin{tabular}{|c|c|}
\hline $\mathrm{C}(2)-\mathrm{C}(3)-\mathrm{C}(23)$ & $120.8(2)$ \\
\hline$C(3)-C(4)-C(5)$ & $120.7(2)$ \\
\hline $\mathrm{C}(3)-\mathrm{C}(4)-\mathrm{C}(17)$ & $120.5(2)$ \\
\hline$C(5)-C(4)-C(17)$ & $118.8(2)$ \\
\hline$C(6)-C(5)-C(10)$ & $118.3(2)$ \\
\hline$C(6)-C(5)-C(4)$ & $122.7(2)$ \\
\hline $\mathrm{C}(10)-\mathrm{C}(5)-\mathrm{C}(4)$ & $119.0(2)$ \\
\hline$C(7)-C(6)-C(5)$ & $120.7(2)$ \\
\hline $\mathrm{C}(7)-\mathrm{C}(6)-\mathrm{H}(6)$ & 119.7 \\
\hline $\mathrm{C}(5)-\mathrm{C}(6)-\mathrm{H}(6)$ & 119.7 \\
\hline$C(6)-C(7)-C(8)$ & 121.1(2) \\
\hline$C(6)-C(7)-S(1)$ & $120.2(2)$ \\
\hline $\mathrm{C}(8)-\mathrm{C}(7)-\mathrm{S}(1)$ & $118.60(19)$ \\
\hline $\mathrm{C}(9)-\mathrm{C}(8)-\mathrm{C}(7)$ & $119.7(2)$ \\
\hline $\mathrm{C}(9)-\mathrm{C}(8)-\mathrm{H}(8)$ & 120.2 \\
\hline $\mathrm{C}(7)-\mathrm{C}(8)-\mathrm{H}(8)$ & 120.2 \\
\hline $\mathrm{C}(8)-\mathrm{C}(9)-\mathrm{C}(10)$ & $121.3(2)$ \\
\hline $\mathrm{C}(8)-\mathrm{C}(9)-\mathrm{H}(9)$ & 119.4 \\
\hline $\mathrm{C}(10)-\mathrm{C}(9)-\mathrm{H}(9)$ & 119.4 \\
\hline $\mathrm{C}(1)-\mathrm{C}(10)-\mathrm{C}(9)$ & $121.1(2)$ \\
\hline$C(1)-C(10)-C(5)$ & $120.0(2)$ \\
\hline $\mathrm{C}(9)-\mathrm{C}(10)-\mathrm{C}(5)$ & $118.9(2)$ \\
\hline$C(16)-C(11)-C(12)$ & $118.4(3)$ \\
\hline $\mathrm{C}(16)-\mathrm{C}(11)-\mathrm{C}(1)$ & $122.1(2)$ \\
\hline$C(12)-C(11)-C(1)$ & $119.3(2)$ \\
\hline $\mathrm{C}(11)-\mathrm{C}(12)-\mathrm{C}(13)$ & $121.2(3)$ \\
\hline $\mathrm{C}(11)-\mathrm{C}(12)-\mathrm{H}(12)$ & 119.4 \\
\hline $\mathrm{C}(13)-\mathrm{C}(12)-\mathrm{H}(12)$ & 119.4 \\
\hline$C(14)-C(13)-C(12)$ & $119.4(3)$ \\
\hline $\mathrm{C}(14)-\mathrm{C}(13)-\mathrm{H}(13)$ & 120.3 \\
\hline $\mathrm{C}(12)-\mathrm{C}(13)-\mathrm{H}(13)$ & 120.3 \\
\hline $\mathrm{C}(15)-\mathrm{C}(14)-\mathrm{C}(13)$ & $120.1(3)$ \\
\hline $\mathrm{C}(15)-\mathrm{C}(14)-\mathrm{H}(14)$ & 119.9 \\
\hline $\mathrm{C}(13)-\mathrm{C}(14)-\mathrm{H}(14)$ & 119.9 \\
\hline$C(14)-C(15)-C(16)$ & $120.0(3)$ \\
\hline $\mathrm{C}(14)-\mathrm{C}(15)-\mathrm{H}(15)$ & 120.0 \\
\hline $\mathrm{C}(16)-\mathrm{C}(15)-\mathrm{H}(15)$ & 120.0 \\
\hline$C(11)-C(16)-C(15)$ & $120.9(3)$ \\
\hline
\end{tabular}




\begin{tabular}{|c|c|}
\hline $\mathrm{C}(11)-\mathrm{C}(16)-\mathrm{H}(16)$ & 119.5 \\
\hline $\mathrm{C}(15)-\mathrm{C}(16)-\mathrm{H}(16)$ & 119.5 \\
\hline $\mathrm{C}(22)-\mathrm{C}(17)-\mathrm{C}(18)$ & $118.3(3)$ \\
\hline$C(22)-C(17)-C(4)$ & $120.4(2)$ \\
\hline $\mathrm{C}(18)-\mathrm{C}(17)-\mathrm{C}(4)$ & $121.3(2)$ \\
\hline $\mathrm{C}(19)-\mathrm{C}(18)-\mathrm{C}(17)$ & $121.3(3)$ \\
\hline C(19)-C(18)-H(18) & 119.4 \\
\hline $\mathrm{C}(17)-\mathrm{C}(18)-\mathrm{H}(18)$ & 119.4 \\
\hline $\mathrm{C}(18)-\mathrm{C}(19)-\mathrm{C}(20)$ & 119.7(3) \\
\hline $\mathrm{C}(18)-\mathrm{C}(19)-\mathrm{H}(19)$ & 120.2 \\
\hline $\mathrm{C}(20)-\mathrm{C}(19)-\mathrm{H}(19)$ & 120.2 \\
\hline $\mathrm{C}(21)-\mathrm{C}(20)-\mathrm{C}(19)$ & 120.1(3) \\
\hline $\mathrm{C}(21)-\mathrm{C}(20)-\mathrm{H}(20)$ & 119.9 \\
\hline $\mathrm{C}(19)-\mathrm{C}(20)-\mathrm{H}(20)$ & 119.9 \\
\hline$C(20)-C(21)-C(22)$ & $120.4(3)$ \\
\hline $\mathrm{C}(20)-\mathrm{C}(21)-\mathrm{H}(21)$ & 119.8 \\
\hline $\mathrm{C}(22)-\mathrm{C}(21)-\mathrm{H}(21)$ & 119.8 \\
\hline $\mathrm{C}(17)-\mathrm{C}(22)-\mathrm{C}(21)$ & 120.2(3) \\
\hline $\mathrm{C}(17)-\mathrm{C}(22)-\mathrm{H}(22)$ & 119.9 \\
\hline $\mathrm{C}(21)-\mathrm{C}(22)-\mathrm{H}(22)$ & 119.9 \\
\hline $\mathrm{C}(3)-\mathrm{C}(23)-\mathrm{C}(24)$ & $112.3(2)$ \\
\hline $\mathrm{C}(3)-\mathrm{C}(23)-\mathrm{P}(1)$ & $113.79(16)$ \\
\hline $\mathrm{C}(24)-\mathrm{C}(23)-\mathrm{P}(1)$ & $111.26(17)$ \\
\hline $\mathrm{C}(3)-\mathrm{C}(23)-\mathrm{H}(23)$ & 106.3 \\
\hline $\mathrm{C}(24)-\mathrm{C}(23)-\mathrm{H}(23)$ & 106.3 \\
\hline $\mathrm{P}(1)-\mathrm{C}(23)-\mathrm{H}(23)$ & 106.3 \\
\hline $\mathrm{C}(23)-\mathrm{C}(24)-\mathrm{H}(24 \mathrm{~A})$ & 109.5 \\
\hline $\mathrm{C}(23)-\mathrm{C}(24)-\mathrm{H}(24 \mathrm{~B})$ & 109.5 \\
\hline $\mathrm{H}(24 \mathrm{~A})-\mathrm{C}(24)-\mathrm{H}(24 \mathrm{~B})$ & 109.5 \\
\hline $\mathrm{C}(23)-\mathrm{C}(24)-\mathrm{H}(24 \mathrm{C})$ & 109.5 \\
\hline $\mathrm{H}(24 \mathrm{~A})-\mathrm{C}(24)-\mathrm{H}(24 \mathrm{C})$ & 109.5 \\
\hline $\mathrm{H}(24 \mathrm{~B})-\mathrm{C}(24)-\mathrm{H}(24 \mathrm{C})$ & 109.5 \\
\hline$C(26)-C(25)-C(30)$ & $118.4(2)$ \\
\hline$C(26)-C(25)-P(1)$ & $117.8(2)$ \\
\hline $\mathrm{C}(30)-\mathrm{C}(25)-\mathrm{P}(1)$ & $123.7(2)$ \\
\hline$C(25)-C(26)-C(27)$ & $120.5(3)$ \\
\hline $\mathrm{C}(25)-\mathrm{C}(26)-\mathrm{H}(26)$ & 119.7 \\
\hline $\mathrm{C}(27)-\mathrm{C}(26)-\mathrm{H}(26)$ & 119.7 \\
\hline
\end{tabular}




\begin{tabular}{|c|c|}
\hline $\mathrm{C}(28)-\mathrm{C}(27)-\mathrm{C}(26)$ & $120.2(3)$ \\
\hline $\mathrm{C}(28)-\mathrm{C}(27)-\mathrm{H}(27)$ & 119.9 \\
\hline $\mathrm{C}(26)-\mathrm{C}(27)-\mathrm{H}(27)$ & 119.9 \\
\hline $\mathrm{C}(27)-\mathrm{C}(28)-\mathrm{C}(29)$ & $120.0(3)$ \\
\hline $\mathrm{C}(27)-\mathrm{C}(28)-\mathrm{H}(28)$ & 120.0 \\
\hline $\mathrm{C}(29)-\mathrm{C}(28)-\mathrm{H}(28)$ & 120.0 \\
\hline $\mathrm{C}(28)-\mathrm{C}(29)-\mathrm{C}(30)$ & $120.2(3)$ \\
\hline $\mathrm{C}(28)-\mathrm{C}(29)-\mathrm{H}(29)$ & 119.9 \\
\hline $\mathrm{C}(30)-\mathrm{C}(29)-\mathrm{H}(29)$ & 119.9 \\
\hline $\mathrm{C}(29)-\mathrm{C}(30)-\mathrm{C}(25)$ & $120.6(3)$ \\
\hline $\mathrm{C}(29)-\mathrm{C}(30)-\mathrm{H}(30)$ & 119.7 \\
\hline $\mathrm{C}(25)-\mathrm{C}(30)-\mathrm{H}(30)$ & 119.7 \\
\hline $\mathrm{C}(32)-\mathrm{C}(31)-\mathrm{C}(36)$ & 118.1(3) \\
\hline $\mathrm{C}(32)-\mathrm{C}(31)-\mathrm{P}(1)$ & $123.6(2)$ \\
\hline $\mathrm{C}(36)-\mathrm{C}(31)-\mathrm{P}(1)$ & $118.3(2)$ \\
\hline $\mathrm{C}(31)-\mathrm{C}(32)-\mathrm{C}(33)$ & $120.7(3)$ \\
\hline $\mathrm{C}(31)-\mathrm{C}(32)-\mathrm{H}(32)$ & 119.6 \\
\hline $\mathrm{C}(33)-\mathrm{C}(32)-\mathrm{H}(32)$ & 119.6 \\
\hline $\mathrm{C}(34)-\mathrm{C}(33)-\mathrm{C}(32)$ & $120.4(3)$ \\
\hline $\mathrm{C}(34)-\mathrm{C}(33)-\mathrm{H}(33)$ & 119.8 \\
\hline $\mathrm{C}(32)-\mathrm{C}(33)-\mathrm{H}(33)$ & 119.8 \\
\hline $\mathrm{C}(33)-\mathrm{C}(34)-\mathrm{C}(35)$ & $119.7(3)$ \\
\hline $\mathrm{C}(33)-\mathrm{C}(34)-\mathrm{H}(34)$ & 120.1 \\
\hline $\mathrm{C}(35)-\mathrm{C}(34)-\mathrm{H}(34)$ & 120.1 \\
\hline $\mathrm{C}(34)-\mathrm{C}(35)-\mathrm{C}(36)$ & $120.5(3)$ \\
\hline $\mathrm{C}(34)-\mathrm{C}(35)-\mathrm{H}(35)$ & 119.7 \\
\hline $\mathrm{C}(36)-\mathrm{C}(35)-\mathrm{H}(35)$ & 119.7 \\
\hline $\mathrm{C}(35)-\mathrm{C}(36)-\mathrm{C}(31)$ & $120.5(3)$ \\
\hline $\mathrm{C}(35)-\mathrm{C}(36)-\mathrm{H}(36)$ & 119.8 \\
\hline $\mathrm{C}(31)-\mathrm{C}(36)-\mathrm{H}(36)$ & 119.8 \\
\hline $\mathrm{C}(38)-\mathrm{C}(37)-\mathrm{C}(42)$ & $120.3(3)$ \\
\hline $\mathrm{C}(38)-\mathrm{C}(37)-\mathrm{S}(1)$ & $119.8(2)$ \\
\hline $\mathrm{C}(42)-\mathrm{C}(37)-\mathrm{S}(1)$ & $119.7(2)$ \\
\hline $\mathrm{C}(39)-\mathrm{C}(38)-\mathrm{C}(37)$ & 119.7(3) \\
\hline $\mathrm{C}(39)-\mathrm{C}(38)-\mathrm{H}(38)$ & 120.2 \\
\hline $\mathrm{C}(37)-\mathrm{C}(38)-\mathrm{H}(38)$ & 120.2 \\
\hline $\mathrm{C}(38)-\mathrm{C}(39)-\mathrm{C}(40)$ & $121.0(3)$ \\
\hline $\mathrm{C}(38)-\mathrm{C}(39)-\mathrm{H}(39)$ & 119.5 \\
\hline
\end{tabular}




\begin{tabular}{|c|c|}
\hline $\mathrm{C}(40)-\mathrm{C}(39)-\mathrm{H}(39)$ & 119.5 \\
\hline $\mathrm{C}(39)-\mathrm{C}(40)-\mathrm{C}(41)$ & $118.2(3)$ \\
\hline $\mathrm{C}(39)-\mathrm{C}(40)-\mathrm{C}(43)$ & $121.2(3)$ \\
\hline $\mathrm{C}(41)-\mathrm{C}(40)-\mathrm{C}(43)$ & $120.6(3)$ \\
\hline $\mathrm{C}(42)-\mathrm{C}(41)-\mathrm{C}(40)$ & $121.0(3)$ \\
\hline $\mathrm{C}(42)-\mathrm{C}(41)-\mathrm{H}(41)$ & 119.5 \\
\hline $\mathrm{C}(40)-\mathrm{C}(41)-\mathrm{H}(41)$ & 119.5 \\
\hline $\mathrm{C}(41)-\mathrm{C}(42)-\mathrm{C}(37)$ & $119.8(3)$ \\
\hline $\mathrm{C}(41)-\mathrm{C}(42)-\mathrm{H}(42)$ & 120.1 \\
\hline $\mathrm{C}(37)-\mathrm{C}(42)-\mathrm{H}(42)$ & 120.1 \\
\hline $\mathrm{C}(40)-\mathrm{C}(43)-\mathrm{H}(43 \mathrm{~A})$ & 109.5 \\
\hline $\mathrm{C}(40)-\mathrm{C}(43)-\mathrm{H}(43 \mathrm{~B})$ & 109.5 \\
\hline $\mathrm{H}(43 \mathrm{~A})-\mathrm{C}(43)-\mathrm{H}(43 \mathrm{~B})$ & 109.5 \\
\hline $\mathrm{C}(40)-\mathrm{C}(43)-\mathrm{H}(43 \mathrm{C})$ & 109.5 \\
\hline $\mathrm{H}(43 \mathrm{~A})-\mathrm{C}(43)-\mathrm{H}(43 \mathrm{C})$ & 109.5 \\
\hline $\mathrm{H}(43 \mathrm{~B})-\mathrm{C}(43)-\mathrm{H}(43 \mathrm{C})$ & 109.5 \\
\hline $\mathrm{C}(2)-\mathrm{O}(1)-\mathrm{H}(1)$ & 109.5 \\
\hline $\mathrm{O}(2)-\mathrm{P}(1)-\mathrm{C}(31)$ & $111.71(12)$ \\
\hline $\mathrm{O}(2)-\mathrm{P}(1)-\mathrm{C}(25)$ & $111.72(11)$ \\
\hline C(31)-P(1)-C(25) & $107.95(12)$ \\
\hline $\mathrm{O}(2)-\mathrm{P}(1)-\mathrm{C}(23)$ & $111.06(11)$ \\
\hline $\mathrm{C}(31)-\mathrm{P}(1)-\mathrm{C}(23)$ & $106.86(12)$ \\
\hline $\mathrm{C}(25)-\mathrm{P}(1)-\mathrm{C}(23)$ & $107.28(12)$ \\
\hline $\mathrm{O}(3)-\mathrm{S}(1)-\mathrm{O}(4)$ & $119.65(14)$ \\
\hline $\mathrm{O}(3)-\mathrm{S}(1)-\mathrm{C}(37)$ & $110.40(13)$ \\
\hline $\mathrm{O}(4)-\mathrm{S}(1)-\mathrm{C}(37)$ & $107.63(13)$ \\
\hline $\mathrm{O}(3)-\mathrm{S}(1)-\mathrm{C}(7)$ & $107.80(12)$ \\
\hline $\mathrm{O}(4)-\mathrm{S}(1)-\mathrm{C}(7)$ & $107.00(13)$ \\
\hline $\mathrm{C}(37)-\mathrm{S}(1)-\mathrm{C}(7)$ & $103.07(12)$ \\
\hline
\end{tabular}

Symmetry transformations used to generate equivalent atoms:

Table S10. Hydrogen bonds for 4 .

\begin{tabular}{lcccc}
\hline $\mathrm{D}-\mathrm{H} \ldots \mathrm{A}$ & $\mathrm{d}(\mathrm{D}-\mathrm{H})$ & $\mathrm{d}(\mathrm{H} \ldots \mathrm{A})$ & $\mathrm{d}(\mathrm{D} \ldots \mathrm{A})$ & $<(\mathrm{DHA})$ \\
$\mathrm{C}(24)-\mathrm{H}(24 \mathrm{~A}) \ldots \mathrm{O}(1)$ & 0.96 & 2.54 & $3.137(3)$ & 120.8 \\
$\mathrm{O}(1)-\mathrm{H}(1) \ldots \mathrm{O}(2)$ & 0.82 & 1.93 & $2.606(2)$ & 138.6 \\
\hline
\end{tabular}

Symmetry transformations used to generate equivalent atoms: 


\section{Characterizations of Substrates and Products}

\subsection{Substrates}

All of the vinyl enyne substrates were prepared according to the procedures reported by our previous works: L. Wu et. al., Adv. Synth. Catal. 2018, 360, 3518-3525; L. Wu et. al., Org. Lett. 2019, 21, 6383-6387. For new compounds, please see characterizations as follows:

(3-(di-p-tolylmethylene)-5-phenylpent-1-en-4-yn-2-yl) diphenylphosphine oxide (10a)

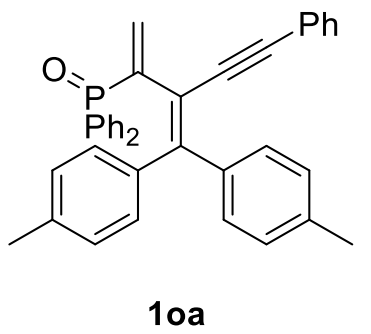

A white solid, (192 mg, 60\% yield), m.p.: $218.4-220.9{ }^{\circ} \mathrm{C}$. Eluent: petroleum ether/ethyl acetate $=1: 1 .{ }^{1} \mathbf{H}$ NMR $\left(400 \mathrm{MHz}, \mathrm{CDCl}_{3}\right) \delta 7.87-$ $7.80(\mathrm{~m}, 4 \mathrm{H}), 7.56-7.50(\mathrm{~m}, 2 \mathrm{H}), 7.48-7.43(\mathrm{~m}, 4 \mathrm{H}), 7.35(\mathrm{~d}, J=8.0 \mathrm{~Hz}$, 2H), $7.22-7.11(\mathrm{~m}, 5 \mathrm{H}), 7.04(\mathrm{~d}, J=7.9 \mathrm{~Hz}, 2 \mathrm{H}), 6.94(\mathrm{~d}, J=7.9 \mathrm{~Hz}, 2 \mathrm{H})$, $6.75(\mathrm{~d}, J=7.0 \mathrm{~Hz}, 2 \mathrm{H}), 6.13(\mathrm{~d}, J=31.6 \mathrm{~Hz}, 1 \mathrm{H}), 6.06(\mathrm{~d}, J=11.7 \mathrm{~Hz}$, 1H), $2.36(\mathrm{~s}, 3 \mathrm{H}), 2.34(\mathrm{~s}, 3 \mathrm{H}) .{ }^{13} \mathbf{C}$ NMR $\left(101 \mathrm{MHz}, \mathrm{CDCl}_{3}\right) \delta 152.4(\mathrm{~d}, J$ $=7.0 \mathrm{~Hz}), 143.1,142.1,138.8,137.9,137.2,136.1(\mathrm{~d}, J=7.5 \mathrm{~Hz}), 132.7$, 132.2, $132.2(\mathrm{~d}, J=103.0 \mathrm{~Hz}), 132.1,131.8$ (d, $J=3.0 \mathrm{~Hz}), 131.1,130.3$, $130.2,128.5,128.4,128.2,128.2,127.9,127.9,123.2,116.7$ (d, $J=9.1$ $\mathrm{Hz}), 95.5,90.4,21.4,21.3 .{ }^{31} \mathbf{P}$ NMR (162 MHz, $\left.\mathrm{CDCl}_{3}\right) \delta 27.76$ (s). HRMS (ESI): $\left([\mathrm{M}+\mathrm{H}]^{+}\right)$Calcd for $\mathrm{C}_{38} \mathrm{H}_{32} \mathrm{OP}^{+}:$535.2185, Found: 535.2180. IR (film) v 3051, 2922, 2188, 1966, 1715, 1508, 1434, 1192, 1113, 980, $812,691 \mathrm{~cm}^{-1}$. 
(3-(bis(4-chlorophenyl)methylene)-5-phenylpent-1-en-4-yn-2-yl) diphenylphosphine oxide (1pa)<smiles>C#CC(=C(C(=C)C#Cc1ccccc1)c1ccc(Cl)cc1)c1ccc(Cl)cc1</smiles>

A white solid, (230 mg, 67\% yield), m.p.: 209.7 - $211.7{ }^{\circ} \mathrm{C}$. Eluent: petroleum ether/ethyl acetate $=1: 1 .{ }^{1} \mathbf{H}$ NMR $\left(400 \mathrm{MHz}, \mathrm{CDCl}_{3}\right) \delta 7.84-$ $7.72(\mathrm{~m}, 4 \mathrm{H}), 7.56-7.50(\mathrm{~m}, 2 \mathrm{H}), 7.49-7.43(\mathrm{~m}, 4 \mathrm{H}), 7.37(\mathrm{~d}, J=8.5 \mathrm{~Hz}$, 2H), $7.31-7.27(\mathrm{~m}, 2 \mathrm{H}), 7.25-7.20(\mathrm{~m}, 3 \mathrm{H}), 7.16(\mathrm{~m}, 2 \mathrm{H}), 7.09(\mathrm{~d}, J=$ $8.4 \mathrm{~Hz}, 2 \mathrm{H}), 6.73(\mathrm{~d}, J=7.0 \mathrm{~Hz}, 2 \mathrm{H}), 6.08(\mathrm{~d}, J=38.3 \mathrm{~Hz}, 1 \mathrm{H}), 5.94(\mathrm{~d}, J$ $=18.2 \mathrm{~Hz}, 1 \mathrm{H}) .{ }^{13} \mathbf{C ~ N M R}\left(101 \mathrm{MHz}, \mathrm{CDCl}_{3}\right) \delta 149.4(\mathrm{~d}, J=6.8 \mathrm{~Hz}), 143.0$, $142.1,139.5,138.7,136.1(\mathrm{~d}, J=8.1 \mathrm{~Hz}), 134.1,133.8,132.1,132.0,131.9$, $131.8(\mathrm{~d}, J=104.0 \mathrm{~Hz}), 131.6,131.2,128.5,128.4,128.3,128.0,127.9$, 122.6, $118.8(\mathrm{~d}, J=8.6 \mathrm{~Hz}), 96.8,89.3 .{ }^{31} \mathbf{P}$ NMR $\left(162 \mathrm{MHz}, \mathrm{CDCl}_{3}\right) \delta$ 27.94 (s). HRMS (ESI): $\left([\mathrm{M}+\mathrm{H}]^{+}\right)$Calcd for $\mathrm{C}_{36} \mathrm{H}_{26} \mathrm{Cl}_{2} \mathrm{OP}^{+}: 575.1093$, Found: 575.1090. IR (film) v 3051, 2192, 1966, 1600, 1505, 1434, 1221, $1186,980,826,690 \mathrm{~cm}^{-1}$.

\section{(3-(bis(4-fluorophenyl)methylene)-5-phenylpent-1-en-4-yn-2-yl)diph-} enylphosphine oxide (1qa)<smiles></smiles>

A white solid, (254 mg, 78\% yield), m.p.: 203.1 - $205.0{ }^{\circ} \mathrm{C}$. Eluent: petroleum ether/ethyl acetate $=1: 1 .{ }^{1} \mathbf{H}$ NMR $\left(400 \mathrm{MHz}, \mathrm{CDCl}_{3}\right) \delta 7.83-$ 
$7.75(\mathrm{~m}, 4 \mathrm{H}), 7.57-7.49(\mathrm{~m}, 2 \mathrm{H}), 7.48-7.38(\mathrm{~m}, 6 \mathrm{H}), 7.23-7.18(\mathrm{~m}$, 1H), $7.18-7.10(\mathrm{~m}, 4 \mathrm{H}), 7.04-6.92(\mathrm{~m}, 4 \mathrm{H}), 6.73(\mathrm{~d}, J=7.1 \mathrm{~Hz}, 2 \mathrm{H})$, $6.08(\mathrm{~d}, J=38.4 \mathrm{~Hz}, 1 \mathrm{H}), 5.93(\mathrm{~d}, J=18.3 \mathrm{~Hz}, 1 \mathrm{H}) .{ }^{13} \mathbf{C} \mathbf{N M R}(101 \mathrm{MHz}$, $\left.\mathrm{CDCl}_{3}\right) \delta 163.6(\mathrm{~d}, J=18.3 \mathrm{~Hz}), 161.1(\mathrm{~d}, J=18.2 \mathrm{~Hz}), 149.9(\mathrm{~d}, J=6.8$ $\mathrm{Hz}), 143.2,142.2,137.3$ (d, $J=3.5 \mathrm{~Hz}), 136.4,136.0$ (d, $J=8.0 \mathrm{~Hz}), 132.3$, 132.2, 132.1, 132.0, $131.9(\mathrm{~d}, J=3.0 \mathrm{~Hz}), 131.9(\mathrm{~d}, J=104.0 \mathrm{~Hz}), 131.1$, $128.5,128.3,128.3,128.0,122.7,115.1,114.9,114.7,114.5,96.1,89.6$. ${ }^{19}$ F NMR (376 MHz, $\left.\mathrm{CDCl}_{3}\right) \delta-112.84$ (s), -113.69 (s). ${ }^{31}$ P NMR (162 $\left.\mathrm{MHz}, \mathrm{CDCl}_{3}\right) \delta 27.97$ (s). HRMS (ESI): $\left([\mathrm{M}+\mathrm{H}]^{+}\right)$Calcd for $\mathrm{C}_{36} \mathrm{H}_{26} \mathrm{~F}_{2} \mathrm{OP}^{+}$: 543.1684, Found: 543.1680. IR (film) v 3080, 2922, 2180, 1966, 1590, $1488,1434,1188,1114,1088,913,818,689 \mathrm{~cm}^{-1}$.

(3-(diphenylmethylene)-5-(naphthalen-1-yl)pent-1-en-4-yn-2-yl)diphenylphosphine oxide (1 ka)

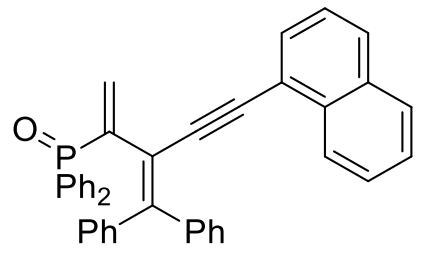

1 ka

A white solid, $\left(260 \mathrm{mg}, 78 \%\right.$ yield), m.p.: 195.7 - $197.4{ }^{\circ} \mathrm{C}$. Eluent: petroleum ether/ethyl acetate=1:1. ${ }^{1} \mathbf{H}$ NMR $\left(400 \mathrm{MHz}, \mathrm{CDCl}_{3}\right) \delta 7.94-$ $7.83(\mathrm{~m}, 4 \mathrm{H}), 7.79-7.73(\mathrm{~m}, 1 \mathrm{H}), 7.63-7.55(\mathrm{~m}, 4 \mathrm{H}), 7.54-7.41(\mathrm{~m}$, $8 \mathrm{H}), 7.36(\mathrm{~d}, J=6.7 \mathrm{~Hz}, 3 \mathrm{H}), 7.27(\mathrm{~d}, J=3.8 \mathrm{~Hz}, 3 \mathrm{H}), 7.21-7.14(\mathrm{~m}, 2 \mathrm{H})$, $7.10(\mathrm{~s}, 1 \mathrm{H}), 6.82(\mathrm{~d}, J=8.4 \mathrm{~Hz}, 1 \mathrm{H}), 6.13(\mathrm{~d}, J=38.3 \mathrm{~Hz}, 1 \mathrm{H}), 6.02(\mathrm{~d}, J$ $=18.2 \mathrm{~Hz}, 1 \mathrm{H}) .{ }^{13} \mathbf{C} \mathbf{N M R}\left(101 \mathrm{MHz}, \mathrm{CDCl}_{3}\right) \delta 152.5(\mathrm{~d}, J=7.1 \mathrm{~Hz}), 142.9$, 142.0, 141.6, 140.7, 136.1 (d, $J=7.8 \mathrm{~Hz}), 132.7,132.3,132.2,132.2$ (d, $J$ $=109.1 \mathrm{~Hz}), 131.9(\mathrm{~d}, J=2.6 \mathrm{~Hz}), 131.1,130.4,130.2,128.5,128.4,128.1$, $128.0,127.9,127.7,127.7,127.6,127.6,127.5,126.6,126.4,120.3,117.9$ (d, $J=8.9 \mathrm{~Hz}), 96.5,90.3 .{ }^{31} \mathbf{P}$ NMR $\left(162 \mathrm{MHz}, \mathrm{CDCl}_{3}\right) \delta 28.07$ (s). HRMS 
(ESI): $\left([\mathrm{M}+\mathrm{H}]^{+}\right)$Calcd for $\mathrm{C}_{40} \mathrm{H}_{30} \mathrm{OP}^{+}$: 557.2029, Found: 557.2027. IR (film) v 3050, 2199, 1908, 1594, 1489, 1435, 1188, 1121, 955, 821, 694 $\mathrm{cm}^{-1}$.

\subsection{Products}

(3-(hydroxydiphenylmethyl)-5-phenyl-5-tosylpenta-1,3,4-trien-2yl)diphenylphosphine oxide (3aa)<smiles>C=C(C=CC(=O)C(O)(c1ccccc1)c1ccccc1)C(=O)S(=O)(=O)c1ccc(C)cc1</smiles>

3aa

A white solid, (109 mg, 81\% yield), m.p.: $173.8-175.2{ }^{\circ} \mathrm{C}$. Eluent: petroleum ether/ethyl acetate $=2.5: 1 .{ }^{1} \mathbf{H}$ NMR $\left(400 \mathrm{MHz}, \mathrm{CDCl}_{3}\right) \delta 7.95$ $(\mathrm{d}, J=7.4 \mathrm{~Hz}, 2 \mathrm{H}), 7.82(\mathrm{~s}, 1 \mathrm{H}), 7.65-7.57(\mathrm{~m}, 2 \mathrm{H}), 7.56-7.49(\mathrm{~m}, 2 \mathrm{H})$, $7.49-7.44(\mathrm{~m}, 3 \mathrm{H}), 7.44-7.41(\mathrm{~m}, 2 \mathrm{H}), 7.41-7.34(\mathrm{~m}, 4 \mathrm{H}), 7.25(\mathrm{~s}, 1 \mathrm{H})$, $7.24-7.19(\mathrm{~m}, 2 \mathrm{H}), 7.19-7.14(\mathrm{~m}, 2 \mathrm{H}), 7.13-7.10(\mathrm{~m}, 2 \mathrm{H}), 7.08(\mathrm{~d}, J=$ $2.0 \mathrm{~Hz}, 2 \mathrm{H}), 7.06-7.00(\mathrm{~m}, 2 \mathrm{H}), 6.57$ (d, $J=7.4 \mathrm{~Hz}, 2 \mathrm{H}), 6.45$ (d, $J=10.1$ $\mathrm{Hz}, 1 \mathrm{H}), 5.51(\mathrm{~d}, J=19.0 \mathrm{~Hz}, 1 \mathrm{H}), 2.36$ (s, 3H). ${ }^{13} \mathrm{C}$ NMR (101 MHz, $\left.\mathrm{CDCl}_{3}\right) \delta 205.0(\mathrm{~d}, J=7.0 \mathrm{~Hz}), 145.7,144.2,143.1,138.0(\mathrm{~d}, J=91.9 \mathrm{~Hz})$, 136.9 (d, $J=10.4 \mathrm{~Hz}), 136.7,132.6(\mathrm{~d}, J=3.0 \mathrm{~Hz}), 132.3$ (d, $J=3.0 \mathrm{~Hz})$, $132.1(\mathrm{~d}, J=11.1 \mathrm{~Hz}), 131.8(\mathrm{~d}, J=10.0 \mathrm{~Hz}), 130.0(\mathrm{~d}, J=107.1 \mathrm{~Hz})$, 129.4, 128.7, 128.7, $128.6(\mathrm{~d}, J=56.6 \mathrm{~Hz}), 128.6(\mathrm{~d}, J=6.1 \mathrm{~Hz}), 128.5(\mathrm{~d}$, $J=9.1 \mathrm{~Hz}), 128.1,128.1$ (d, $J=49.5 \mathrm{~Hz}), 128.0,128.0,127.9,127.7,127.3$, $127.3(\mathrm{~d}, J=26.3 \mathrm{~Hz}), 120.8(\mathrm{~d}, J=7.8 \mathrm{~Hz}), 115.4,80.9,21.6 .{ }^{31} \mathbf{P}$ NMR $\left(162 \mathrm{MHz}, \mathrm{CDCl}_{3}\right) \delta 35.69$ (s). HRMS (ESI): $\left([\mathrm{M}+\mathrm{Na}]^{+}\right)$Calcd for $\mathrm{C}_{43} \mathrm{H}_{35} \mathrm{NaO}_{4} \mathrm{PS}^{+}:$701.1886, Found: 701.1882. IR (film) v 3182, 3060, 1945, 1593, 1443, 1303, 1144, 1085, 814, 756, $692 \mathrm{~cm}^{-1}$. 
(3-(hydroxydiphenylmethyl)-5-phenyl-5-(phenylsulfonyl)penta-1,3,4trien-2-yl)diphenylphosphine oxide (3ab)<smiles>C=C(C(=CC(=O)c1ccccc1)C(O)(c1ccccc1)c1ccccc1)c1ccccc1</smiles>

\section{$3 a b$}

A white solid, (86 mg, 65\% yield), m.p.: $150.1-151.5{ }^{\circ} \mathrm{C}$. Eluent: petroleum ether/ethyl acetate $=2.5: 1 .{ }^{1} \mathbf{H}$ NMR $\left(400 \mathrm{MHz}, \mathrm{CDCl}_{3}\right) \delta 7.98-$ $7.92(\mathrm{~m}, 2 \mathrm{H}), 7.82-7.81(\mathrm{~m}, 1 \mathrm{H}), 7.64-7.59(\mathrm{~m}, 2 \mathrm{H}), 7.55-7.50(\mathrm{~m}$, 2H), $7.49-7.47(\mathrm{~m}, 2 \mathrm{H}), 7.46-7.41(\mathrm{~m}, 4 \mathrm{H}), 7.38-7.36(\mathrm{~m}, 3 \mathrm{H}), 7.35-$ $7.27(\mathrm{~m}, 4 \mathrm{H}), 7.24-7.15(\mathrm{~m}, 3 \mathrm{H}), 7.14-7.07(\mathrm{~m}, 2 \mathrm{H}), 7.06-7.00(\mathrm{~m}$, 3H), 6.54 (d, J = 7.2 Hz, 2H), $6.44(\mathrm{~d}, \mathrm{~J}=39.7 \mathrm{~Hz}, 1 \mathrm{H}), 5.52$ (d, J = 19.0 $\mathrm{Hz}, 1 \mathrm{H}) .{ }^{13} \mathbf{C} \mathbf{N M R}\left(101 \mathrm{MHz}, \mathrm{CDCl}_{3}\right) \delta 205.1(\mathrm{~d}, \mathrm{~J}=7.0 \mathrm{~Hz}), 145.7,143.1$, 139.6, 137.9 (d, $J=101.1 \mathrm{~Hz}), 137.0$ (d, J = 10.3 Hz), 133.3, 132.6 (d, J = 3.0 Hz), 132.5, 132.3 (d, J = 3.0 Hz), 132.2 (d, J = 10.0 Hz), 131.8 (d, J = $10.0 \mathrm{~Hz}), 130.1,130.0$ (d, $J=106.1 \mathrm{~Hz}), 128.9,128.8,128.6$ (d, $J=3.0$ $\mathrm{Hz}), 128.5$ (d, $J=10.1 \mathrm{~Hz}), 128.3,128.2$ (d, $J=3.0 \mathrm{~Hz}), 128.1,128.1$, 128.0, 127.9, 127.7, 127.3 (d, $J=30.3 \mathrm{~Hz}), 127.3,120.9$ (d, J = 7.7 Hz), 115.3, 81.0. ${ }^{31} \mathbf{P}$ NMR (162 MHz, $\left.\mathrm{CDCl}_{3}\right) \delta 35.68$ (s). HRMS (ESI): $\left([\mathrm{M}+\mathrm{H}]^{+}\right) \mathrm{C}_{42} \mathrm{H}_{34} \mathrm{O}_{4} \mathrm{PS}^{+}:$665.1910, Found: 665.1891. IR (film) v 3190, 3060, 1946, 1596, 1440, 1303, 1144, 1082, 814, 756, 692.

(3-(hydroxydiphenylmethyl)-5-((4-methoxyphenyl)sulfonyl)-5-phenyl penta-1,3,4-trien-2-yl)diphenylphosphine oxide (3ac) 


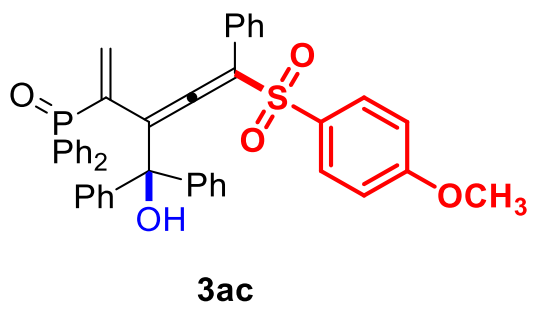

A white solid, (104 mg, 75\% yield), m.p.: 167.7 - $169.5{ }^{\circ} \mathrm{C}$. Eluent: petroleum ether/ethyl acetate=2.5:1. ${ }^{1} \mathbf{H}$ NMR $\left(400 \mathrm{MHz}, \mathrm{CDCl}_{3}\right) \delta 7.95$ $(\mathrm{d}, J=7.5 \mathrm{~Hz}, 2 \mathrm{H}), 7.82(\mathrm{~s}, 1 \mathrm{H}), 7.65-7.60(\mathrm{~m}, 2 \mathrm{H}), 7.55-7.46(\mathrm{~m}, 3 \mathrm{H})$, $7.45-7.39(\mathrm{~m}, 5 \mathrm{H}), 7.38-7.34(\mathrm{~m}, 2 \mathrm{H}), 7.30-7.26(\mathrm{~m}, 2 \mathrm{H}), 7.24-7.15$ $(\mathrm{m}, 3 \mathrm{H}), 7.15-6.98(\mathrm{~m}, 5 \mathrm{H}), 6.76(\mathrm{~d}, J=8.9 \mathrm{~Hz}, 2 \mathrm{H}), 6.55(\mathrm{~d}, J=7.4 \mathrm{~Hz}$, $2 \mathrm{H}), 6.45(\mathrm{~d}, J=39.8 \mathrm{~Hz}, 1 \mathrm{H}), 5.51(\mathrm{~d}, J=19.0 \mathrm{~Hz}, 1 \mathrm{H}), 3.80(\mathrm{~s}, 3 \mathrm{H}) \cdot{ }^{13} \mathbf{C}$ NMR $\left(101 \mathrm{MHz}, \mathrm{CDCl}_{3}\right) \delta 204.7(\mathrm{~d}, J=7.0 \mathrm{~Hz}), 163.4,145.7,143.2$, $138.0(\mathrm{~d}, J=91.9 \mathrm{~Hz}), 136.9(\mathrm{~d}, J=10.3 \mathrm{~Hz}), 132.5(\mathrm{~d}, J=3.0 \mathrm{~Hz}), 132.3$ (d, $J=3.0 \mathrm{~Hz}), 132.1$ (d, $J=10.3 \mathrm{~Hz}), 131.8(\mathrm{~d}, J=10.0 \mathrm{~Hz}), 131.2,130.2$, $130.0(\mathrm{~d}, J=107.1 \mathrm{~Hz}), 128.8,128.8(\mathrm{~d}, J=53.5 \mathrm{~Hz}), 128.7,128.6(\mathrm{~d}, J=$ $5.1 \mathrm{~Hz}), 128.5(\mathrm{~d}, J=10.3 \mathrm{~Hz}), 128.1,128.0,128.0,127.6,127.4,127.3$ (d, $J=25.2 \mathrm{~Hz}), 120.6(\mathrm{~d}, J=7.7 \mathrm{~Hz}), 115.7,113.9,80.9,55.6 .{ }^{31} \mathbf{P}$ NMR $(162$ $\left.\mathrm{MHz}, \mathrm{CDCl}_{3}\right) \delta 35.61$ (s). HRMS (ESI): $\left([\mathrm{M}+\mathrm{H}]^{+}\right)$Calcd for $\mathrm{C}_{43} \mathrm{H}_{36} \mathrm{O}_{5} \mathrm{PS}^{+}$: 695.2016, Found: 695.2005. IR (film) v 3186, 3058, 1955, 1595, 1497, $1311,1266,1141,1086,1025,831,757,693 \mathrm{~cm}^{-1}$.

(5-((4-(tert-butyl)phenyl)sulfonyl)-3-(hydroxydiphenylmethyl)-5phenylpenta-1,3,4-trien-2-yl)diphenylphosphine oxide (3ad)<smiles>C=C(C(=CC(=O)c1ccccc1)C(O)(c1ccccc1)c1ccccc1)c1ccc(Br)cc1</smiles>

3ad

A white solid, (101 mg, 70\% yield), m.p.: 186.2 - $188.0{ }^{\circ} \mathrm{C}$. Eluent: petroleum ether/ethyl acetate $=2.5: 1 .{ }^{1} \mathbf{H}$ NMR $\left(400 \mathrm{MHz}, \mathrm{CDCl}_{3}\right) \delta 7.96$ 
$(\mathrm{d}, J=7.5 \mathrm{~Hz}, 2 \mathrm{H}), 7.81(\mathrm{~s}, 1 \mathrm{H}), 7.66-7.58(\mathrm{~m}, 2 \mathrm{H}), 7.56-7.49(\mathrm{~m}, 2 \mathrm{H})$, $7.46-7.40(\mathrm{~m}, 5 \mathrm{H}), 7.35(\mathrm{~d}, J=7.7 \mathrm{~Hz}, 4 \mathrm{H}), 7.31(\mathrm{~d}, J=2.2 \mathrm{~Hz}, 3 \mathrm{H}), 7.23$ $-7.15(\mathrm{~m}, 3 \mathrm{H}), 7.10-6.98(\mathrm{~m}, 5 \mathrm{H}), 6.53$ (d, $J=7.4 \mathrm{~Hz}, 2 \mathrm{H}), 6.44(\mathrm{~d}, J=$ $39.8 \mathrm{~Hz}, 1 \mathrm{H}), 5.51$ (d, $J=19.0 \mathrm{~Hz}, 1 \mathrm{H}), 1.30$ (s, 9H). ${ }^{13} \mathbf{C}$ NMR $(101 \mathrm{MHz}$, $\left.\mathrm{CDCl}_{3}\right) \delta 205.2(\mathrm{~d}, J=6.9 \mathrm{~Hz}), 157.2,145.7,143.1,137.8(\mathrm{~d}, J=91.9 \mathrm{~Hz})$, 136.9 (d, $J=9.1 \mathrm{~Hz}), 136.8,132.6(\mathrm{~d}, J=2.8 \mathrm{~Hz}), 132.3(\mathrm{~d}, J=2.0 \mathrm{~Hz})$, $132.0(\mathrm{~d}, J=10.1 \mathrm{~Hz}), 131.8(\mathrm{~d}, J=10.0 \mathrm{~Hz}), 130.0(\mathrm{~d}, J=106.1 \mathrm{~Hz})$, 128.9, 128.8, $128.6(\mathrm{~d}, J=8.1 \mathrm{~Hz}), 128.5(\mathrm{~d}, J=10.1 \mathrm{~Hz}), 128.1,127.9$, $127.9,127.7,127.6,127.2(\mathrm{~d}, J=39.4 \mathrm{~Hz}), 127.2,125.8,120.5$ (d, $J=7.6$ $\mathrm{Hz}), 115.5,80.9,35.2,31.0 .{ }^{31} \mathbf{P}$ NMR (162 MHz, $\left.\mathrm{CDCl}_{3}\right) \delta 35.64$ (s). HRMS (ESI): $\left([\mathrm{M}+\mathrm{H}]^{+}\right)$Calcd for $\mathrm{C}_{46} \mathrm{H}_{42} \mathrm{O}_{4} \mathrm{PS}^{+}$: 721.2536, Found: 721.2539. IR (film) v 3061, 2959, 2799, 1922, 1593, 1448, 1302, 1163 , $1046,962,832,727,693 \mathrm{~cm}^{-1}$.

(5-((4-bromophenyl)sulfonyl)-3-(hydroxydiphenylmethyl)-5-phenyl penta-1,3,4-trien-2-yl)diphenylphosphine oxide (3ae)

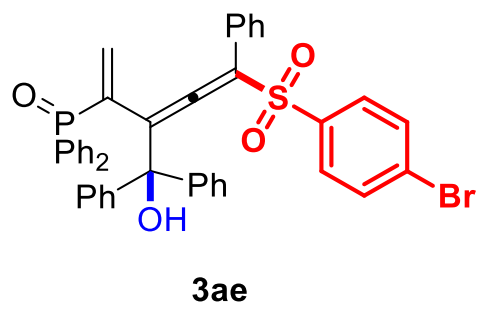

A white solid, (133 mg, 90\% yield), m.p.: 155.5 - $157.5{ }^{\circ} \mathrm{C}$. Eluent: petroleum ether/ethyl acetate $=2.5: 1 .{ }^{1} \mathbf{H}$ NMR $\left(400 \mathrm{MHz}, \mathrm{CDCl}_{3}\right) \delta 7.93$ $(\mathrm{d}, J=7.7 \mathrm{~Hz}, 2 \mathrm{H}), 7.85(\mathrm{~s}, 1 \mathrm{H}), 7.65-7.57(\mathrm{~m}, 2 \mathrm{H}), 7.55-7.46(\mathrm{~m}, 3 \mathrm{H})$, $7.46-7.41$ (m, 5H), $7.41-7.33(\mathrm{~m}, 5 \mathrm{H}), 7.24-7.16(\mathrm{~m}, 3 \mathrm{H}), 7.16(\mathrm{~d}, J=$ $8.5 \mathrm{~Hz}, 2 \mathrm{H}), 7.13-7.03$ (m, 4H), 6.61 (d, $J=7.8 \mathrm{~Hz}, 2 \mathrm{H}), 6.44$ (d, $J=39.6$ $\mathrm{Hz}, 1 \mathrm{H}), 5.52(\mathrm{~d}, J=18.9 \mathrm{~Hz}, 1 \mathrm{H}) .{ }^{13} \mathbf{C}$ NMR $\left(101 \mathrm{MHz}, \mathrm{CDCl}_{3}\right) \delta 205.3$ $(\mathrm{d}, J=6.9 \mathrm{~Hz}), 145.6,143.0,138.6,137.9(\mathrm{~d}, J=90.9 \mathrm{~Hz}), 137.1(\mathrm{~d}, J=$ $10.4 \mathrm{~Hz}), 132.6(\mathrm{~d}, J=2.7 \mathrm{~Hz}), 132.4(\mathrm{~d}, J=3.0 \mathrm{~Hz}), 132.1$ (d, $J=10.1$ 
Hz), 132.0, 131.8 (d, $J=10.0 \mathrm{~Hz}), 129.9$ (d, $J=102.0 \mathrm{~Hz}), 129.4,128.9$, $128.8(\mathrm{~d}, J=5.1 \mathrm{~Hz}), 128.7,128.6(\mathrm{~d}, J=3.0 \mathrm{~Hz}), 128.5(\mathrm{~d}, J=4.0 \mathrm{~Hz})$, $128.2,128.1,128.1,127.9$ (d, $J=17.2 \mathrm{~Hz}), 127.7,127.4,127.4$ (d, $J=20.2$ $\mathrm{Hz}), 121.3(\mathrm{~d}, J=7.7 \mathrm{~Hz}), 114.9,81.0 .{ }^{31} \mathbf{P}$ NMR $\left(162 \mathrm{MHz}, \mathrm{CDCl}_{3}\right) \delta$ 35.60 (s). HRMS (ESI): $\left([\mathrm{M}+\mathrm{H}]^{+}\right)$Calcd for $\mathrm{C}_{42} \mathrm{H}_{33} \mathrm{BrO}_{4} \mathrm{PS}^{+}:$: 743.1015, Found: 743.1008. IR (film) v 3232, 3060, 1936, 1572, 1435, 1388, 1315, $1174,1030,955,824,693 \mathrm{~cm}^{-1}$.

(5-((4-fluorophenyl)sulfonyl)-3-(hydroxydiphenylmethyl)-5-phenyl penta-1,3,4-trien-2-yl)diphenylphosphine oxide (3af)

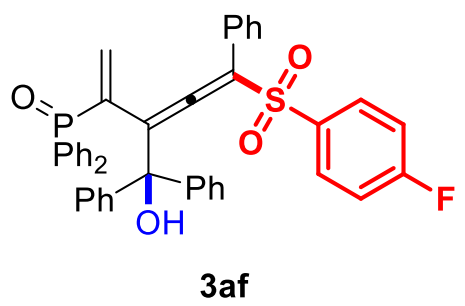

A white solid, (106 mg, 78\% yield), m.p.: $169.1-170.5{ }^{\circ} \mathrm{C}$. Eluent: petroleum ether/ethyl acetate $=2.5: 1 .{ }^{1} \mathbf{H}$ NMR $\left(400 \mathrm{MHz}, \mathrm{CDCl}_{3}\right) \delta 7.93$ $(\mathrm{d}, J=7.3 \mathrm{~Hz}, 2 \mathrm{H}), 7.86(\mathrm{~s}, 1 \mathrm{H}), 7.65-7.57(\mathrm{~m}, 2 \mathrm{H}), 7.56-7.52(\mathrm{~m}, 1 \mathrm{H})$, $7.48(\mathrm{~d}, J=7.3 \mathrm{~Hz}, 1 \mathrm{H}), 7.46-7.41(\mathrm{~m}, 4 \mathrm{H}), 7.40-7.34(\mathrm{~m}, 4 \mathrm{H}), 7.33-$ $7.28(\mathrm{~m}, 2 \mathrm{H}), 7.24-7.18(\mathrm{~m}, 3 \mathrm{H}), 7.14(\mathrm{~d}, J=7.0 \mathrm{~Hz}, 1 \mathrm{H}), 7.12-7.03(\mathrm{~m}$, 4H), $7.00-6.92(\mathrm{~m}, 2 \mathrm{H}), 6.58(\mathrm{~d}, J=7.3 \mathrm{~Hz}, 2 \mathrm{H}), 6.44(\mathrm{~d}, J=39.6 \mathrm{~Hz}$, $1 \mathrm{H}), 5.51(\mathrm{~d}, J=18.9 \mathrm{~Hz}, 1 \mathrm{H}) .{ }^{13} \mathbf{C} \mathbf{N M R}\left(101 \mathrm{MHz}, \mathrm{CDCl}_{3}\right) \delta 205.0(\mathrm{~d}, J$ $=7.0 \mathrm{~Hz}), 145.6,143.0,137.9(\mathrm{~d}, J=92.9 \mathrm{~Hz}), 137.1(\mathrm{~d}, J=10.5 \mathrm{~Hz})$, $135.5(\mathrm{~d}, J=3.0 \mathrm{~Hz}), 132.6(\mathrm{~d}, J=2.8 \mathrm{~Hz}), 132.3$ (d, $J=2.0 \mathrm{~Hz}), 132.1(\mathrm{~d}$, $J=11.2 \mathrm{~Hz}), 131.8(\mathrm{~d}, J=9.9 \mathrm{~Hz}), 130.8(\mathrm{~d}, J=9.5 \mathrm{~Hz}), 129.9(\mathrm{~d}, J=$ $107.1 \mathrm{~Hz}), 128.9,128.8,128.7,128.6(\mathrm{~d}, J=5.1 \mathrm{~Hz}), 128.5,128.1,128.1$, 128.1, 127.9, 127.7, 127.4, 127.4 (d, $J=22.2 \mathrm{~Hz}), 121.2(\mathrm{~d}, J=8.2 \mathrm{~Hz})$, 116.2, 115.9, 115.0, 80.9. ${ }^{19} \mathbf{F}$ NMR $\left(376 \mathrm{MHz}, \mathrm{CDCl}_{3}\right) \delta-103.69$ (s). ${ }^{31} \mathbf{P}$ NMR (162 MHz, $\left.\mathrm{CDCl}_{3}\right) \delta 35.55$ (s). HRMS (ESI): ([M+H] $\left.]^{+}\right)$Calcd for 
$\mathrm{C}_{42} \mathrm{H}_{33} \mathrm{FO}_{4} \mathrm{PS}^{+}:$683.1816, Found: 683.1824. IR (film) v 3235, 3062, 1940, $1588,1491,1323,1173,1144,1033,837,694 \mathrm{~cm}^{-1}$.

(3-(hydroxydiphenylmethyl)-5-phenyl-5-((4-(trifluoromethyl)phenyl) sulfonyl)penta-1,3,4-trien-2-yl)diphenylphosphine oxide (3ag)

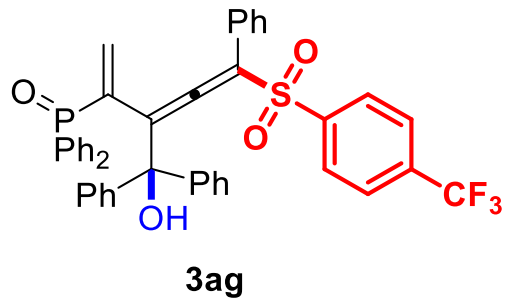

A white solid, (107 mg, 73\% yield), m.p.: 141.6 - $143.4{ }^{\circ} \mathrm{C}$. Eluent: petroleum ether/ethyl acetate=2.5:1. ${ }^{1} \mathbf{H}$ NMR $\left(400 \mathrm{MHz}, \mathrm{CDCl}_{3}\right)(400$ $\left.\mathrm{MHz}, \mathrm{CDCl}_{3}\right) \delta 7.95-7.87(\mathrm{~m}, 3 \mathrm{H}), 7.65-7.58(\mathrm{~m}, 2 \mathrm{H}), 7.58-7.52(\mathrm{~m}$, $3 \mathrm{H}), 7.51-7.43(\mathrm{~m}, 5 \mathrm{H}), 7.42-7.38(\mathrm{~m}, 4 \mathrm{H}), 7.38-7.34(\mathrm{~m}, 2 \mathrm{H}), 7.25-$ $7.18(\mathrm{~m}, 3 \mathrm{H}), 7.17-7.11(\mathrm{~m}, 1 \mathrm{H}), 7.11-7.03(\mathrm{~m}, 4 \mathrm{H}), 6.68-6.55(\mathrm{~m}$, 2H), $6.45(\mathrm{~d}, J=39.5 \mathrm{~Hz}, 1 \mathrm{H}), 5.53(\mathrm{~d}, J=18.9 \mathrm{~Hz}, 1 \mathrm{H}) .{ }^{13} \mathbf{C}$ NMR $(101$ $\left.\mathrm{MHz}, \mathrm{CDCl}_{3}\right) \delta 205.6(\mathrm{~d}, J=7.1 \mathrm{~Hz}), 145.5,143.1,142.9,137.9(\mathrm{~d}, J=$ $91.9 \mathrm{~Hz}), 137.1(\mathrm{~d}, J=10.1 \mathrm{~Hz}), 134.8$ (d, $J=33.3 \mathrm{~Hz}), 132.7$ (d, $J=3.0$ $\mathrm{Hz}), 132.4(\mathrm{~d}, J=2.0 \mathrm{~Hz}), 132.1(\mathrm{~d}, J=11.1 \mathrm{~Hz}), 131.8(\mathrm{~d}, J=10.0 \mathrm{~Hz})$, $129.8(\mathrm{~d}, J=107.1 \mathrm{~Hz}), 128.9(\mathrm{~d}, J=14.2 \mathrm{~Hz}), 128.7,128.5(\mathrm{~d}, J=12.1$ Hz), 128.3, 128.3, 128.3 (d, $J=104.0 \mathrm{~Hz}), 128.1,128.1,127.7,127.5$ (d, $J$ $=23.2 \mathrm{~Hz}), 127.4,125.9$ (q, $J=3.0 \mathrm{~Hz}), 124.4,121.7$ (d, $J=7.8 \mathrm{~Hz}), 114.5$, 81.0. ${ }^{19}$ F NMR (376 MHz, $\left.\mathrm{CDCl}_{3}\right) \delta-63.17$ (s). ${ }^{31}$ P NMR (162 MHz, $\left.\mathrm{CDCl}_{3}\right) \delta 35.60$ (s). HRMS (ESI): $\left([\mathrm{M}+\mathrm{H}]^{+}\right)$Calcd for $\mathrm{C}_{43} \mathrm{H}_{33} \mathrm{~F}_{3} \mathrm{O}_{4} \mathrm{PS}^{+}$: 733.1784, Found: 733.1773. IR (film) v 3075, 2972, 2811, 1941, 1489, $1320,1120,1062,967,758,691 \mathrm{~cm}^{-1}$.

(3-(hydroxydiphenylmethyl)-5-((4-nitrophenyl)sulfonyl)-5-phenyl penta-1,3,4-trien-2-yl)diphenylphosphine oxide (3ah) 


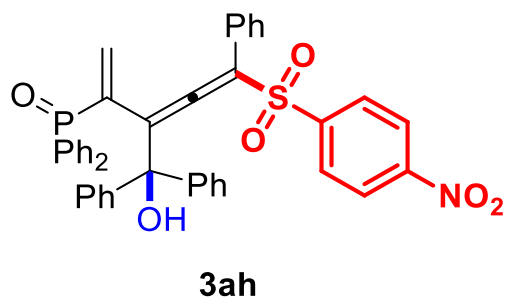

A white solid, (120 mg, 85\% yield), m.p.: $139.9-141.2{ }^{\circ} \mathrm{C}$. Eluent: petroleum ether/ethyl acetate $=2.5: 1 .{ }^{1} \mathbf{H}$ NMR $\left(400 \mathrm{MHz}, \mathrm{CDCl}_{3}\right) \delta 8.10$ $(\mathrm{d}, J=8.8 \mathrm{~Hz}, 2 \mathrm{H}), 7.96-7.86(\mathrm{~m}, 3 \mathrm{H}), 7.64-7.58(\mathrm{~m}, 2 \mathrm{H}), 7.57-7.52$ (m, 1H), $7.48-7.45(\mathrm{~m}, 2 \mathrm{H}), 7.44(\mathrm{~s}, 2 \mathrm{H}), 7.46-7.39(\mathrm{~m}, 5 \mathrm{H}), 7.39-7.34$ (m, 2H), $7.25-7.16(\mathrm{~m}, 4 \mathrm{H}), 7.15-7.05(\mathrm{~m}, 4 \mathrm{H}), 6.66(\mathrm{~d}, J=7.3 \mathrm{~Hz}, 2 \mathrm{H})$, $6.45(\mathrm{~d}, J=39.4 \mathrm{~Hz}, 1 \mathrm{H}), 5.53(\mathrm{~d}, J=18.9 \mathrm{~Hz}, 1 \mathrm{H}) .{ }^{13} \mathbf{C} \mathbf{N M R}(101 \mathrm{MHz}$, $\left.\mathrm{CDCl}_{3}\right) \delta 205.7(\mathrm{~d}, J=7.0 \mathrm{~Hz}), 150.2,145.4,145.2,142.9,137.8(\mathrm{~d}, J=$ $91.9 \mathrm{~Hz}), 137.2(\mathrm{~d}, J=9.1 \mathrm{~Hz}), 132.7(\mathrm{~d}, J=2.9 \mathrm{~Hz}), 132.4(\mathrm{~d}, J=2.8 \mathrm{~Hz})$, $132.1(\mathrm{~d}, J=10.2 \mathrm{~Hz}), 131.8(\mathrm{~d}, J=10.0 \mathrm{~Hz}), 130.2(\mathrm{~d}, J=20.2 \mathrm{~Hz}), 129.2$, 129.1, 128.7 (d, $J=13.1 \mathrm{~Hz}), 128.6$ (d, $J=55.6 \mathrm{~Hz}), 128.6,128.6$ (d, $J=$ 13.1 Hz), 128.4, 128.1, 128.1, 127.8, 127.5 (d, $J=17.2 \mathrm{~Hz}), 127.5,123.9$, $122.1(\mathrm{~d}, J=7.9 \mathrm{~Hz}), 114.2,81.0 .{ }^{31} \mathbf{P}$ NMR $\left(162 \mathrm{MHz}, \mathrm{CDCl}_{3}\right) \delta 35.58(\mathrm{~s})$. HRMS (ESI): $\left([\mathrm{M}+\mathrm{H}]^{+}\right)$Calcd for $\mathrm{C}_{42} \mathrm{H}_{33} \mathrm{NO}_{6} \mathrm{PS}^{+}$: 710.1761, Found: 710.1747. IR (film) v 3234, 3060, 1936, 1573, 1435, 1322, 1174, 1146, $955,758,693 \mathrm{~cm}^{-1}$.

(3-(hydroxydiphenylmethyl)-5-phenyl-5-(m-tolylsulfonyl)penta-1,3,4trien-2-yl)diphenylphosphine oxide (3ai)<smiles>C=C(C=C(C(=O)O)C(O)(c1ccccc1)c1ccccc1)C(=O)S(=O)(=O)c1cccc(C)c1</smiles>

A white solid, (108 mg, 80\% yield), m.p.: 145.1 - $147.0{ }^{\circ} \mathrm{C}$. Eluent: petroleum ether/ethyl acetate $=2.5: 1 .{ }^{1} \mathbf{H}$ NMR $\left(400 \mathrm{MHz}, \mathrm{CDCl}_{3}\right) \delta 7.96$ 
$(\mathrm{d}, J=7.6 \mathrm{~Hz}, 2 \mathrm{H}), 7.79(\mathrm{~s}, 1 \mathrm{H}), 7.65-7.57(\mathrm{~m}, 2 \mathrm{H}), 7.54-7.49(\mathrm{~m}, 2 \mathrm{H})$, $7.47-7.42(\mathrm{~m}, 4 \mathrm{H}), 7.39-7.35(\mathrm{~m}, 5 \mathrm{H}), 7.30(\mathrm{~d}, J=8.9 \mathrm{~Hz}, 1 \mathrm{H}), 7.24-$ $7.21(\mathrm{~m}, 2 \mathrm{H}), 7.19-7.15(\mathrm{~m}, 2 \mathrm{H}), 7.13-7.08(\mathrm{~m}, 2 \mathrm{H}), 7.08-7.00(\mathrm{~m}$, 4H), 6.50 (d, $J=7.4 \mathrm{~Hz}, 2 \mathrm{H}), 6.43(\mathrm{~d}, J=39.8 \mathrm{~Hz}, 1 \mathrm{H}), 5.52$ (d, $J=19.0$ $\mathrm{Hz}, 1 \mathrm{H}), 2.28(\mathrm{~s}, 3 \mathrm{H}) .{ }^{13} \mathbf{C} \mathbf{N M R}\left(101 \mathrm{MHz}, \mathrm{CDCl}_{3}\right) \delta 204.9(\mathrm{~d}, J=6.9 \mathrm{~Hz})$, 145.8, 143.1, 139.5, 138.8, 137.9 (d, $J=92.9 \mathrm{~Hz}), 136.8$ (d, $J=10.4 \mathrm{~Hz})$, 134.1, $132.6(\mathrm{~d}, J=2.7 \mathrm{~Hz}), 132.3(\mathrm{~d}, J=3.0 \mathrm{~Hz}), 132.1(\mathrm{~d}, J=11.1 \mathrm{~Hz})$, $131.8(\mathrm{~d}, J=10.0 \mathrm{~Hz}), 130.0(\mathrm{~d}, J=107.0 \mathrm{~Hz}), 128.8,128.8,128.7$ (d, $J=$ $62.6 \mathrm{~Hz}), 128.7(\mathrm{~d}, J=3.0 \mathrm{~Hz}), 128.6(\mathrm{~d}, J=3.0 \mathrm{~Hz}), 128.3(\mathrm{~d}, J=33.3$ $\mathrm{Hz}), 128.1$ (d, $J=48.5 \mathrm{~Hz}), 128.0,128.0,127.9,127.6,127.3$ (d, $J=32.3$ $\mathrm{Hz}), 127.2,125.2,120.7$ (d, $J=7.8 \mathrm{~Hz}), 115.5,80.9,21.2 .{ }^{31} \mathbf{P}$ NMR (162 $\left.\mathrm{MHz}, \mathrm{CDCl}_{3}\right) \delta 35.64$ (s). HRMS (ESI): $\left([\mathrm{M}+\mathrm{H}]^{+}\right)$Calcd for $\mathrm{C}_{43} \mathrm{H}_{36} \mathrm{O}_{4} \mathrm{PS}^{+}$: 679.2066, Found: 679.2055. IR (film) v 3191, 3061, 1946, 1581, 1436, $1306,1141,1044,898,755,685 \mathrm{~cm}^{-1}$.

\section{(3-(hydroxydiphenylmethyl)-5-(naphthalen-2-ylsulfonyl)-5-phenyl}

penta-1,3,4-trien-2-yl)diphenylphosphine oxide (3aj)

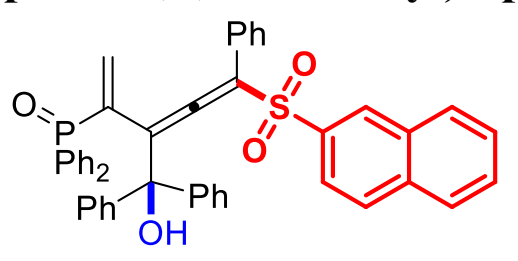

3aj

A white solid, (72 mg, 50\% yield), m.p.: $144.9-146.9{ }^{\circ} \mathrm{C}$. Eluent: petroleum ether/ethyl acetate $=2.5: 1 .{ }^{1} \mathbf{H}$ NMR $\left(400 \mathrm{MHz}, \mathrm{CDCl}_{3}\right) \delta 8.16$ (s, 1H), 7.97 (d, $J=7.5 \mathrm{~Hz}, 2 \mathrm{H}), 7.87-7.80(\mathrm{~m}, 3 \mathrm{H}), 7.72$ (d, $J=8.7 \mathrm{~Hz}$, 1H), 7.65 - $7.56(\mathrm{~m}, 4 \mathrm{H}), 7.55-7.52(\mathrm{~m}, 1 \mathrm{H}), 7.51-7.46(\mathrm{~m}, 3 \mathrm{H}), 7.45-$ $7.41(\mathrm{~m}, 2 \mathrm{H}), 7.40-7.33(\mathrm{~m}, 4 \mathrm{H}), 7.22-7.12(\mathrm{~m}, 4 \mathrm{H}), 7.07-6.95(\mathrm{~m}$, $5 \mathrm{H}), 6.54$ (d, $J=7.4 \mathrm{~Hz}, 2 \mathrm{H}), 6.46(\mathrm{~d}, J=39.7 \mathrm{~Hz}, 1 \mathrm{H}), 5.54$ (d, $J=19.0$ $\mathrm{Hz}, 1 \mathrm{H}) .{ }^{13} \mathbf{C} \mathbf{N M R}\left(101 \mathrm{MHz}, \mathrm{CDCl}_{3}\right) \delta 205.1(\mathrm{~d}, J=6.9 \mathrm{~Hz}), 145.7,143.1$, 
$138.0(\mathrm{~d}, J=92.9 \mathrm{~Hz}), 136.8(\mathrm{~d}, J=11.1 \mathrm{~Hz}), 136.7,135.0,132.6(\mathrm{~d}, J=$ $2.7 \mathrm{~Hz}), 132.3(\mathrm{~d}, J=2.7 \mathrm{~Hz}), 132.1(\mathrm{~d}, J=10.1 \mathrm{~Hz}), 131.8(\mathrm{~d}, J=10.1$ $\mathrm{Hz}), 131.8,130.0$ (d, $J=107.6 \mathrm{~Hz}), 129.4$ (d, $J=25.3 \mathrm{~Hz}), 129.0$ (d, $J=$ $18.2 \mathrm{~Hz}), 128.8,128.7$ (d, $J=13.1 \mathrm{~Hz}), 128.5$ (d, $J=12.1 \mathrm{~Hz}), 128.1(\mathrm{~d}, J$ $=42.4 \mathrm{~Hz}), 128.1,128.0,128.0,127.6,127.5(\mathrm{~d}, J=69.7 \mathrm{~Hz}), 127.5,127.3$, $122.8,120.8(\mathrm{~d}, J=7.6 \mathrm{~Hz}), 115.5,81.0 .{ }^{31} \mathbf{P}$ NMR $\left(162 \mathrm{MHz}, \mathrm{CDCl}_{3}\right) \delta$ 35.63 (s). HRMS (ESI): $\left([\mathrm{M}+\mathrm{H}]^{+}\right)$Calcd for $\mathrm{C}_{46} \mathrm{H}_{36} \mathrm{O}_{4} \mathrm{PS}^{+}$: 715.2066, Found: 715.2057. IR (film) v 3195, 3054, 2920, 2849, 1954, 1489, 1436, $1313,1145,1126,812,693 \mathrm{~cm}^{-1}$.

\section{(5-((5-(dimethylamino)naphthalen-1-yl)sulfonyl)-3-(hydroxydiphenyl} methyl)-5-phenylpenta-1,3,4-trien-2-yl)diphenylphosphine oxide (3ak)<smiles>C=C(C(=O)O)C(=CC(=CC(=O)[PH2+])C(O)(c1ccccc1)c1ccccc1)S(=O)(=O)c1cccc2c(N(C)C)cccc12</smiles>

3ak

A white solid, (92 mg, 61\% yield), m.p.: $179.7-181.7{ }^{\circ} \mathrm{C}$. Eluent: petroleum ether/ethyl acetate $=2.5: 1 .{ }^{1} \mathbf{H}$ NMR $\left(400 \mathrm{MHz}, \mathrm{CDCl}_{3}\right) \delta 8.50$ $(\mathrm{d}, J=8.5 \mathrm{~Hz}, 1 \mathrm{H}), 8.23(\mathrm{~d}, J=8.6 \mathrm{~Hz}, 1 \mathrm{H}), 7.94(\mathrm{~d}, J=7.4 \mathrm{~Hz}, 2 \mathrm{H}), 7.77$ $(\mathrm{d}, J=7.3 \mathrm{~Hz}, 1 \mathrm{H}), 7.69(\mathrm{~s}, 1 \mathrm{H}), 7.64-7.57(\mathrm{~m}, 2 \mathrm{H}), 7.55-7.46(\mathrm{~m}, 3 \mathrm{H})$, $7.46-7.39(\mathrm{~m}, 4 \mathrm{H}), 7.38-7.30(\mathrm{~m}, 6 \mathrm{H}), 7.18-7.10(\mathrm{~m}, 4 \mathrm{H}), 7.08-7.04$ (m, 2H), $6.93-6.84(\mathrm{~m}, 2 \mathrm{H}), 6.25-6.05(\mathrm{~m}, 3 \mathrm{H}), 5.47(\mathrm{~d}, J=19.0 \mathrm{~Hz}$, 1H), $2.89(\mathrm{~s}, 6 \mathrm{H}) .{ }^{13} \mathbf{C}$ NMR $\left(101 \mathrm{MHz}, \mathrm{CDCl}_{3}\right) \delta 203.8(\mathrm{~d}, J=6.7 \mathrm{~Hz})$, 151.8, 145.8, 142.8, 137.6 (d, $J=92.9 \mathrm{~Hz}), 135.9$ (d, $J=10.0 \mathrm{~Hz}), 135.3$, $132.6(\mathrm{~d}, J=2.7 \mathrm{~Hz}), 132.3(\mathrm{~d}, J=2.7 \mathrm{~Hz}), 132.1(\mathrm{~d}, J=10.0 \mathrm{~Hz}), 132.0$ $(\mathrm{d}, J=10.0 \mathrm{~Hz}), 131.0,130.6,130.5$ (d, $J=105.0 \mathrm{~Hz}), 129.9$ (d, $J=57.6$ $\mathrm{Hz}), 128.8,128.6(\mathrm{~d}, J=11.1 \mathrm{~Hz}), 128.4,128.2,128.1,128.0,127.7,127.3$, $127.2(\mathrm{~d}, J=25.3 \mathrm{~Hz}), 123.2,120.0(\mathrm{~d}, J=6.9 \mathrm{~Hz}), 119.5,116.8,115.1$, 
81.2, 45.4. ${ }^{31} \mathbf{P}$ NMR $\left(162 \mathrm{MHz}, \mathrm{CDCl}_{3}\right) \delta 35.67$ (s). HRMS (ESI): $\left([\mathrm{M}+\mathrm{Na}]^{+}\right)$Calcd for $\mathrm{C}_{48} \mathrm{H}_{40} \mathrm{NNaO}_{4} \mathrm{PS}^{+}:$780.2308, Found: 780.2288. IR (film) v 3167, 3057, 2932, 2854, 1957, 1681, 1436, 1305, 1157, 1135, 1050, $796,691 \mathrm{~cm}^{-1}$.

\section{(3-(hydroxydiphenylmethyl)-5-phenyl-5-(thiophen-2-ylsulfonyl)}

penta-1,3,4-trien-2-yl)diphenylphosphine oxide (3al)

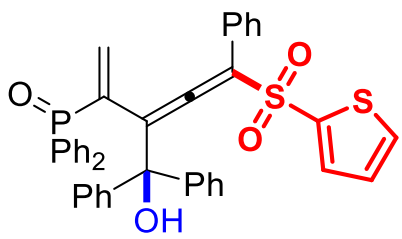

3al

A white solid, (97 mg, 72\% yield), m.p.: $150.5-151.8{ }^{\circ} \mathrm{C}$. Eluent: petroleum ether/ethyl acetate $=2.5: 1 .{ }^{1} \mathbf{H}$ NMR $\left(400 \mathrm{MHz}, \mathrm{CDCl}_{3}\right) \delta 7.95$ $(\mathrm{d}, J=7.4 \mathrm{~Hz}, 2 \mathrm{H}), 7.75(\mathrm{~s}, 1 \mathrm{H}), 7.65-7.58(\mathrm{~m}, 2 \mathrm{H}), 7.57-7.46(\mathrm{~m}, 4 \mathrm{H})$, $7.45-7.41(\mathrm{~m}, 3 \mathrm{H}), 7.40-7.35(\mathrm{~m}, 3 \mathrm{H}), 7.35-7.31(\mathrm{~m}, 1 \mathrm{H}), 7.25-7.14$ (m, 4H), $7.10-7.01(\mathrm{~m}, 5 \mathrm{H}), 6.96-6.92(\mathrm{~m}, 1 \mathrm{H}), 6.48(\mathrm{~d}, J=7.5 \mathrm{~Hz}, 2 \mathrm{H})$, $6.38(\mathrm{~d}, J=39.7 \mathrm{~Hz}, 1 \mathrm{H}), 5.51(\mathrm{~d}, J=18.9 \mathrm{~Hz}, 1 \mathrm{H}) .{ }^{13} \mathbf{C}$ NMR $(101 \mathrm{MHz}$, $\left.\mathrm{CDCl}_{3}\right) \delta 204.5(\mathrm{~d}, J=6.9 \mathrm{~Hz}), 145.7,143.1,140.6,137.7(\mathrm{~d}, J=91.9 \mathrm{~Hz})$, $136.6(\mathrm{~d}, J=10.3 \mathrm{~Hz}), 134.1,133.8,132.6(\mathrm{~d}, J=2.7 \mathrm{~Hz}), 132.4(\mathrm{~d}, J=$ $3.0 \mathrm{~Hz}), 132.1(\mathrm{~d}, J=10.1 \mathrm{~Hz}), 131.8(\mathrm{~d}, J=10.1 \mathrm{~Hz}), 129.9$ (d, $J=107.1$ $\mathrm{Hz}), 128.9,128.9,128.8$ (d, $J=4.0 \mathrm{~Hz}), 128.6$ (d, $J=9.1 \mathrm{~Hz}), \quad 128.4$, 128.1, 128.0, 127.9, 127.8, 127.7, 127.5, $127.3(\mathrm{~d}, J=33.3 \mathrm{~Hz}), 127.0$, $120.6(\mathrm{~d}, J=7.6 \mathrm{~Hz}), 116.2,80.9 .{ }^{31} \mathbf{P}$ NMR $\left(162 \mathrm{MHz}, \mathrm{CDCl}_{3}\right) \delta 35.51$ (s). HRMS (ESI): $\left([\mathrm{M}+\mathrm{H}]^{+}\right)$Calcd for $\mathrm{C}_{40} \mathrm{H}_{32} \mathrm{O}_{4} \mathrm{PS}_{2}{ }^{+}:$671.1474, Found: 671.1483. IR (film) v 3202, 3062, 2921, 1941, 1583, 1437, 1398, 1313, $1141,952,760,693 \mathrm{~cm}^{-1}$. 
(5-(ethylsulfonyl)-3-(hydroxydiphenylmethyl)-5-phenylpenta-1,3,4trien-2-yl)diphenylphosphine oxide (3am)

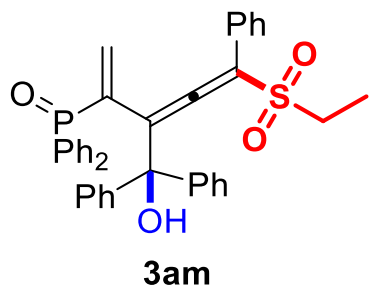

A white solid, (74 mg, 60\% yield), m.p.: 159.2 - $160.9{ }^{\circ} \mathrm{C}$. Eluent: petroleum ether/ethyl acetate $=2.5: 1 .{ }^{1} \mathbf{H}$ NMR $\left(400 \mathrm{MHz}, \mathrm{CDCl}_{3}\right) \delta 7.93$ (s, 1H), $7.87(\mathrm{~d}, J=7.3 \mathrm{~Hz}, 2 \mathrm{H}), 7.63-7.56(\mathrm{~m}, 2 \mathrm{H}), 7.55-7.47(\mathrm{~m}, 3 \mathrm{H})$, $7.46-7.40 \mathrm{~m}, 3 \mathrm{H}), 7.40-7.36(\mathrm{~m}, 3 \mathrm{H}), 7.35-7.24(\mathrm{~m}, 3 \mathrm{H}), 7.21-7.18$ (m, 3H), $7.17-7.14(\mathrm{~m}, 3 \mathrm{H}), 6.93(\mathrm{~d}, J=7.4 \mathrm{~Hz}, 2 \mathrm{H}), 6.44(\mathrm{~d}, J=39.8$ Hz, 1H), 5.46 (d, $J=19.0 \mathrm{~Hz}, 1 \mathrm{H}), 2.74-2.63(\mathrm{~m}, 1 \mathrm{H}), 2.57-2.45$ (m, 1H), $1.04(\mathrm{t}, J=7.5 \mathrm{~Hz}, 3 \mathrm{H}) .{ }^{13} \mathbf{C}$ NMR $\left(101 \mathrm{MHz}, \mathrm{CDCl}_{3}\right) \delta 206.5(\mathrm{~d}, J=$ 7.2 Hz), 145.4, 143.3, 137.8 (d, $J=91.9 \mathrm{~Hz}), 137.2(\mathrm{~d}, J=9.1 \mathrm{~Hz}), 132.6$ $(\mathrm{d}, J=2.7 \mathrm{~Hz}), 132.3(\mathrm{~d}, J=3.0 \mathrm{~Hz}), 132.1(\mathrm{~d}, J=11.1 \mathrm{~Hz}), 131.7(\mathrm{~d}, J=$ $10.0 \mathrm{~Hz}), 130.1,129.8$ (d, $J=108.1 \mathrm{~Hz}), 128.8$ (d, $J=8.1 \mathrm{~Hz}), 128.6(\mathrm{~d}, J$ $=5.1 \mathrm{~Hz}), 128.5,128.4,128.1(\mathrm{~d}, J=70.7 \mathrm{~Hz}), 128.1,128.0,128.0,127.7$, 127.3, 127.3 (d, $J=4.0 \mathrm{~Hz}), 121.2(\mathrm{~d}, J=7.9 \mathrm{~Hz}), 113.3,80.7,48.2,6.3$. ${ }^{31}$ P NMR $\left(162 \mathrm{MHz}, \mathrm{CDCl}_{3}\right) \delta 35.76$ (s). HRMS (ESI): $\left([\mathrm{M}+\mathrm{H}]^{+}\right)$Calcd for $\mathrm{C}_{38} \mathrm{H}_{34} \mathrm{O}_{4} \mathrm{PS}^{+}$: 617.1910, Found: 617.1914. IR (film) $v$ 3183, 3061, 2924, 1942, 1597, 1492, 1437, 1302, 1130, 1046, 882, 766, $634 \mathrm{~cm}^{-1}$.

(3-(hydroxydiphenylmethyl)-5-phenyl-5-(propylsulfonyl)penta-1,3,4trien-2-yl)diphenylphosphine oxide (3an)<smiles>C=C(C=C(C(=O)[PH](=O)c1ccccc1)C(O)(c1ccccc1)c1ccccc1)C(=O)CCC</smiles>

3an 
A white solid, (82 mg, 65\% yield), m.p.: $146.0-147.9{ }^{\circ} \mathrm{C}$. Eluent: petroleum ether/ethyl acetate $=2.5: 1 .{ }^{1} \mathbf{H}$ NMR $\left(400 \mathrm{MHz}, \mathrm{CDCl}_{3}\right) \delta 7.95$ (s, 1H), $7.89-7.82(\mathrm{~m}, 2 \mathrm{H}), 7.63-7.55(\mathrm{~m}, 2 \mathrm{H}), 7.55-7.48(\mathrm{~m}, 3 \mathrm{H}), 7.45$ $-7.36(\mathrm{~m}, 6 \mathrm{H}), 7.35-7.25(\mathrm{~m}, 3 \mathrm{H}), 7.20-7.15(\mathrm{~m}, 6 \mathrm{H}), 7.01-6.93(\mathrm{~m}$, 2H), $6.45(\mathrm{~d}, J=39.8 \mathrm{~Hz}, 1 \mathrm{H}), 5.46(\mathrm{~d}, J=19.0 \mathrm{~Hz}, 1 \mathrm{H}), 2.67-2.57(\mathrm{~m}$, 1H), $2.43-2.33(\mathrm{~m}, 1 \mathrm{H}), 1.63-1.42(\mathrm{~m}, 2 \mathrm{H}), 0.88(\mathrm{t}, J=7.4 \mathrm{~Hz}, 3 \mathrm{H}) .{ }^{13} \mathbf{C}$ NMR $\left(101 \mathrm{MHz}, \mathrm{CDCl}_{3}\right) \delta 206.5(\mathrm{~d}, J=7.1 \mathrm{~Hz}), 145.4,143.3,137.8(\mathrm{~d}, J$ $=81.8 \mathrm{~Hz}), 137.3,132.6(\mathrm{~d}, J=2.6 \mathrm{~Hz}), 132.3(\mathrm{~d}, J=2.7 \mathrm{~Hz}), 132.1(\mathrm{~d}, J$ $=9.9 \mathrm{~Hz}), 131.7(\mathrm{~d}, J=9.9 \mathrm{~Hz}), 129.8(\mathrm{~d}, J=108.1 \mathrm{~Hz}), 128.7$ (d, $J=7.1$ $\mathrm{Hz}), 128.5$ (d, $J=5.1 \mathrm{~Hz}), 128.5,128.4,128.3$ (d, $J=107.1 \mathrm{~Hz}), 128.1$, 128.1, 128.0, 127.6, 127.4, 127.3 (d, $J=7.1 \mathrm{~Hz}), 121.3(\mathrm{~d}, J=8.1 \mathrm{~Hz})$, 113.6, 80.7, 55.4, 15.4, 13.0. ${ }^{31} \mathbf{P}$ NMR (162 MHz, $\left.\mathrm{CDCl}_{3}\right) \delta 35.77$ (s). HRMS (ESI): $\left([\mathrm{M}+\mathrm{Na}]^{+}\right) \mathrm{C}_{39} \mathrm{H}_{35} \mathrm{NaO}_{4} \mathrm{PS}^{+}: 653.1886$, Found: 653.1877 . IR (film) v 3183, 3058, 2973, 2933, 1941, 1597, 1489, 1439, 1296, 1164, $1129,1049,967,753,691 \mathrm{~cm}^{-1}$.

(5-((2-chloroethyl)sulfonyl)-3-(hydroxydiphenylmethyl)-5-phenyl penta-1,3,4-trien-2-yl)diphenylphosphine oxide (3ao)

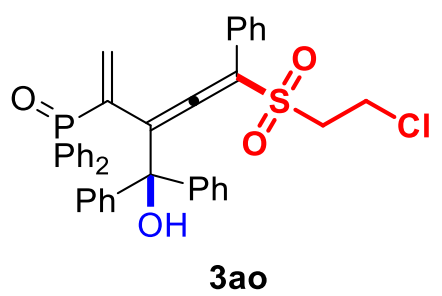

A white solid, (57 mg, 42\% yield), m.p.: 134.0 - $135.8{ }^{\circ} \mathrm{C}$. Eluent: petroleum ether/ethyl acetate $=2.5: 1 .{ }^{1} \mathbf{H}$ NMR $\left(400 \mathrm{MHz}, \mathrm{CDCl}_{3}\right) \delta 7.94$ (s, 1H), $7.86-7.78(\mathrm{~m}, 2 \mathrm{H}), 7.62-7.50(\mathrm{~m}, 5 \mathrm{H}), 7.46-7.37(\mathrm{~m}, 6 \mathrm{H}), 7.37$ $-7.29(\mathrm{~m}, 3 \mathrm{H}), 7.24-7.20(\mathrm{~m}, 4 \mathrm{H}), 7.20-7.16(\mathrm{~m}, 2 \mathrm{H}), 7.04-6.92(\mathrm{~m}$, 2H), $6.41(\mathrm{~d}, J=39.6 \mathrm{~Hz}, 1 \mathrm{H}), 5.47$ (d, $J=19.0 \mathrm{~Hz}, 1 \mathrm{H}), 3.51-3.40(\mathrm{~m}$, 1H), 7.37- $7.25(\mathrm{~m}, 1 \mathrm{H}), 3.11-2.99(\mathrm{~m}, 1 \mathrm{H}), 2.92-2.80(\mathrm{~m}, 1 \mathrm{H}) .{ }^{13} \mathbf{C}$ 
NMR $\left(101 \mathrm{MHz}, \mathrm{CDCl}_{3}\right) \delta 206.7(\mathrm{~d}, J=7.1 \mathrm{~Hz}), 145.1,143.1,137.6(\mathrm{~d}, J$ $=90.9 \mathrm{~Hz}), 137.4(\mathrm{~d}, J=10.1 \mathrm{~Hz}), 132.7(\mathrm{~d}, J=2.8 \mathrm{~Hz}), 132.4(\mathrm{~d}, J=2.8$ $\mathrm{Hz}), 132.1$ (d, $J=10.2 \mathrm{~Hz}), 131.7(\mathrm{~d}, J=10.0 \mathrm{~Hz}), 129.6(\mathrm{~d}, J=107.1 \mathrm{~Hz})$, $129.2,128.7,128.7(\mathrm{~d}, J=12.1 \mathrm{~Hz}), 128.5(\mathrm{~d}, J=12.1 \mathrm{~Hz}), 128.1(\mathrm{~d}, J=$ $31.3 \mathrm{~Hz}), 128.1,128.1,128.0,127.8,127.7$ (d, $J=2.0 \mathrm{~Hz}), 127.6$ (d, $J=$ 13.1 Hz), 127.3, 122.1 (d, $J=8.0 \mathrm{~Hz}), 113.3,80.8,55.5,34.9 .{ }^{31} \mathbf{P}$ NMR $\left(162 \mathrm{MHz}, \mathrm{CDCl}_{3}\right) \delta 35.66$ (s). HRMS (ESI): $\left([\mathrm{M}+\mathrm{Na}]^{+}\right)$Calcd for $\mathrm{C}_{38} \mathrm{H}_{32} \mathrm{ClNaO}_{4} \mathrm{PS}^{+}:$673.1340, Found: 673.1332. IR (film) v 3072, 2922, $2811,1940,1588,1435,1301,1137,967,902,722,691 \mathrm{~cm}^{-1}$.

\section{4-(diphenylphosphoryl)-3-(hydroxydiphenylmethyl)-N,N-dimethyl-1-} phenylpenta-1,2,4-triene-1-sulfonamide (3ap)

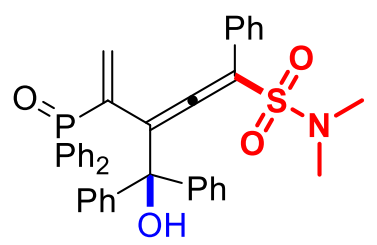

3ap

A white solid, (47 mg, 37\% yield), m.p.: $162.1-164.0{ }^{\circ} \mathrm{C}$. Eluent: petroleum ether/ethyl acetate=2.5:1. ${ }^{1} \mathbf{H}$ NMR $\left(400 \mathrm{MHz}, \mathrm{CDCl}_{3}\right) \delta 7.88$ $(\mathrm{d}, J=7.4 \mathrm{~Hz}, 2 \mathrm{H}), 7.73(\mathrm{~s}, 1 \mathrm{H}), 7.64-7.58(\mathrm{~m}, 2 \mathrm{H}), 7.55-7.50(\mathrm{~m}, 1 \mathrm{H})$, $7.49-7.46(\mathrm{~m}, 1 \mathrm{H}), 7.46-7.41(\mathrm{~m}, 3 \mathrm{H}), 7.41-7.38(\mathrm{~m}, 4 \mathrm{H}), 7.34-7.27$ (m, 2H), $7.27-7.23(\mathrm{~m}, 2 \mathrm{H}), 7.22-7.18(\mathrm{~m}, 3 \mathrm{H}), 7.20-7.11(\mathrm{~m}, 2 \mathrm{H})$, $6.78(\mathrm{~d}, J=7.3 \mathrm{~Hz}, 2 \mathrm{H}), 6.28(\mathrm{~d}, J=39.9 \mathrm{~Hz}, 1 \mathrm{H}), 5.45$ (d, $J=19.0 \mathrm{~Hz}$, 1H), $2.34(\mathrm{~s}, 6 \mathrm{H}) .{ }^{13} \mathrm{C}$ NMR $\left(101 \mathrm{MHz}, \mathrm{CDCl}_{3}\right) \delta 205.3(\mathrm{~d}, J=7.1 \mathrm{~Hz})$, 146.1, 143.0, 138.5 (d, $J=91.9 \mathrm{~Hz}), 136.6$ (d, $J=9.1 \mathrm{~Hz}), 132.5$ (d, $J=$ $2.0 \mathrm{~Hz}), 132.3(\mathrm{~d}, J=3.0 \mathrm{~Hz}), 132.1$ (d, $J=10.1 \mathrm{~Hz}), 131.8$ (d, $J=10.1$ $\mathrm{Hz}), 130.1$ (d, $J=107.1 \mathrm{~Hz}), 129.3(\mathrm{~d}, J=1.0 \mathrm{~Hz}), 128.9,128.7(\mathrm{~d}, J=4.0$ $\mathrm{Hz}), 128.6(\mathrm{~d}, J=4.0 \mathrm{~Hz}), 128.6(\mathrm{~d}, J=107.1 \mathrm{~Hz}), 128.4,128.1,127.9$, 127.7, 127.7, $127.3(\mathrm{~d}, J=6.8 \mathrm{~Hz}), 118.7(\mathrm{~d}, J=8.1 \mathrm{~Hz}), 111.9,80.8,37.7$. 
${ }^{31}$ P NMR (162 MHz, $\left.\mathrm{CDCl}_{3}\right) \delta 35.36$ (s). HRMS (ESI): $\left([\mathrm{M}+\mathrm{Na}]^{+}\right)$Calcd for $\mathrm{C}_{38} \mathrm{H}_{34} \mathrm{NNaO}_{4} \mathrm{PS}^{+}:$654.1838, Found: 654.1830. IR (film) v 3168, 3053, 2972, 1941, 1596, 1439, 1296, 1151, 1117, 1049, 998, 753, $691 \mathrm{~cm}^{-1}$.

\section{(3-(hydroxydiphenylmethyl)-5-(p-tolyl)-5-tosylpenta-1,3,4-trien-2-yl)}

diphenylphosphine oxide (3ba)

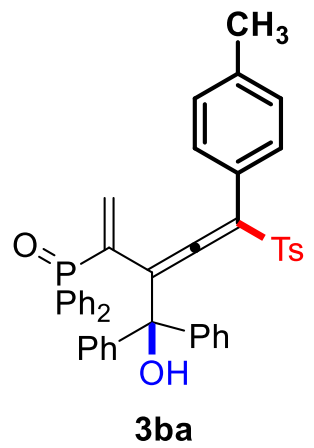

A white solid, (98 mg, 71\% yield), m.p.: $175.7-177.6{ }^{\circ} \mathrm{C}$. Eluent: petroleum ether/ethyl acetate $=2.5: 1 .{ }^{1} \mathbf{H}$ NMR $\left(400 \mathrm{MHz}, \mathrm{CDCl}_{3}\right) \delta 7.95$ $(\mathrm{d}, J=7.4 \mathrm{~Hz}, 2 \mathrm{H}), 7.83(\mathrm{~s}, 1 \mathrm{H}), 7.67-7.59(\mathrm{~m}, 2 \mathrm{H}), 7.54-7.45(\mathrm{~m}, 3 \mathrm{H})$, $7.45-7.40(\mathrm{~m}, 3 \mathrm{H}), 7.40-7.31(\mathrm{~m}, 4 \mathrm{H}), 7.27-7.19(\mathrm{~m}, 4 \mathrm{H}), 7.15-7.04$ (m, 5H), $6.84(\mathrm{~d}, J=8.0 \mathrm{~Hz}, 2 \mathrm{H}), 6.50-6.37$ (m, 3H), $5.50(\mathrm{~d}, J=19.0$ $\mathrm{Hz}, 1 \mathrm{H}), 2.36$ (s, 3H), 2.27 (s, 3H). ${ }^{13} \mathbf{C}$ NMR $\left(101 \mathrm{MHz}, \mathrm{CDCl}_{3}\right) \delta 205.0$ $(\mathrm{d}, J=7.0 \mathrm{~Hz}), 145.7,144.1,143.2,138.5,137.2(\mathrm{~d}, J=74.7 \mathrm{~Hz}), 136.9$ $(\mathrm{d}, J=10.0 \mathrm{~Hz}), 132.5(\mathrm{~d}, J=2.0 \mathrm{~Hz}), 132.3(\mathrm{~d}, J=2.0 \mathrm{~Hz}), 132.1(\mathrm{~d}, J=$ $10.0 \mathrm{~Hz}), 131.8(\mathrm{~d}, J=10.0 \mathrm{~Hz}), 130.1$ (d, $J=106.1 \mathrm{~Hz}), 129.4,129.1$, $128.7,128.7(\mathrm{~d}, J=12.1 \mathrm{~Hz}), 128.5,128.4,128.1,128.0,127.9,127.6$, 127.3, $127.2(\mathrm{~d}, J=26.3 \mathrm{~Hz}), 125.3,120.6(\mathrm{~d}, J=7.8 \mathrm{~Hz}), 115.5,80.9$, 21.6, 21.2. ${ }^{31} \mathbf{P}$ NMR (162 MHz, $\left.\mathrm{CDCl}_{3}\right) \delta 35.64$ (s). HRMS (ESI): $\left([\mathrm{M}+\mathrm{H}]^{+}\right)$Calcd for $\mathrm{C}_{44} \mathrm{H}_{38} \mathrm{O}_{4} \mathrm{PS}^{+}:$693.2223, Found: 693.2215. IR (film) $v$ $3195,3059,2922,1943,1595,1442,1302,1146,1048,845,692 \mathrm{~cm}^{-1}$. 
(3-(hydroxydiphenylmethyl)-5-(4-methoxyphenyl)-5-tosylpenta-1,3,4trien-2-yl)diphenylphosphine oxide (3ca)<smiles>C=C(C(=Cc1ccc(OC)cc1)C(O)(c1ccccc1)c1ccccc1)C(F)(F)c1ccccc1</smiles>

A white solid, (92 mg, 65\% yield), m.p.: $171.0-173.0{ }^{\circ} \mathrm{C}$. Eluent: petroleum ether/ethyl acetate $=2.5: 1 .{ }^{1} \mathbf{H}$ NMR $\left(400 \mathrm{MHz}, \mathrm{CDCl}_{3}\right) \delta 7.95$ $(\mathrm{d}, J=7.8 \mathrm{~Hz}, 2 \mathrm{H}), 7.80(\mathrm{~s}, 1 \mathrm{H}), 7.67-7.57(\mathrm{~m}, 2 \mathrm{H}), 7.54-7.45(\mathrm{~m}, 3 \mathrm{H})$, $7.44-7.40(\mathrm{~m}, 3 \mathrm{H}), 7.40-7.31(\mathrm{~m}, 4 \mathrm{H}), 7.27-7.17(\mathrm{~m}, 4 \mathrm{H}), 7.15-7.01$ $(\mathrm{m}, 5 \mathrm{H}), 6.55(\mathrm{~d}, J=8.8 \mathrm{~Hz}, 2 \mathrm{H}), 6.50-6.33(\mathrm{~m}, 3 \mathrm{H}), 5.50(\mathrm{~d}, J=19.0$ $\mathrm{Hz}, 1 \mathrm{H}), 3.75$ (s, 3H), 2.36 (s, 3H). ${ }^{13} \mathbf{C}$ NMR $\left(101 \mathrm{MHz}, \mathrm{CDCl}_{3}\right) \delta 204.9$ (d, $J=6.9 \mathrm{~Hz}), 159.8,145.8,144.2,143.2,138.0$ (d, $J=92.9 \mathrm{~Hz}), 136.8$, $136.8(\mathrm{~d}, J=10.0 \mathrm{~Hz}), 132.5(\mathrm{~d}, J=2.0 \mathrm{~Hz}), 132.3(\mathrm{~d}, J=2.0 \mathrm{~Hz}), 132.1$ $(\mathrm{d}, J=10.0 \mathrm{~Hz}), 131.8(\mathrm{~d}, J=10.0 \mathrm{~Hz}), 130.1,130.0(\mathrm{~d}, J=107.1 \mathrm{~Hz})$, 129.4, $128.7(\mathrm{~d}, J=12.1 \mathrm{~Hz}), 128.5(\mathrm{~d}, J=107.1 \mathrm{~Hz}), 128.5(\mathrm{~d}, J=12.1$ Hz), 128.1, 128.0, 127.9, 127.6, 127.3, 127.2 (d, $J=29.3 \mathrm{~Hz}), 120.4$ (d, $J$ $=2.0 \mathrm{~Hz}), 120.3(\mathrm{~d}, J=8.1 \mathrm{~Hz}), 115.3,113.4,80.9,55.2,21.6 .{ }^{31}$ P NMR $\left(162 \mathrm{MHz}, \mathrm{CDCl}_{3}\right) \delta 35.74$ (s). HRMS (ESI): $\left([\mathrm{M}+\mathrm{Na}]^{+}\right)$Calcd for $\mathrm{C}_{44} \mathrm{H}_{37} \mathrm{NaO}_{5} \mathrm{PS}^{+}:$731.1992, Found: 731.1981. IR (film) $v 3281,3059,2922$, $1938,1596,1508,1434,1319,1145,1032,764,698 \mathrm{~cm}^{-1}$.

(5-(4-(tert-butyl)phenyl)-3-(hydroxydiphenylmethyl)-5-tosylpenta-1,3, 4-trien-2-yl)diphenylphosphine oxide (3da) 


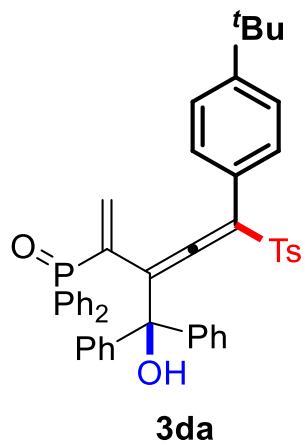

A white solid, (107 mg, 71\% yield), m.p.: $182.7-184.6{ }^{\circ} \mathrm{C}$. Eluent: petroleum ether/ethyl acetate $=2.5: 1 .{ }^{1} \mathbf{H}$ NMR $\left(400 \mathrm{MHz}, \mathrm{CDCl}_{3}\right) \delta 7.95$ $(\mathrm{d}, J=7.5 \mathrm{~Hz}, 2 \mathrm{H}), 7.85(\mathrm{~s}, 1 \mathrm{H}), 7.65-7.56(\mathrm{~m}, 2 \mathrm{H}), 7.53-7.46(\mathrm{~m}, 2 \mathrm{H})$, $7.45-7.39(\mathrm{~m}, 6 \mathrm{H}), 7.37-7.30(\mathrm{~m}, 2 \mathrm{H}), 7.26(\mathrm{~d}, J=8.2 \mathrm{~Hz}, 2 \mathrm{H}), 7.18-$ $7.11(\mathrm{~m}, 4 \mathrm{H}), 7.10-7.02(\mathrm{~m}, 5 \mathrm{H}), 6.57(\mathrm{~d}, J=8.4 \mathrm{~Hz}, 2 \mathrm{H}), 6.46(\mathrm{~d}, J=$ $39.9 \mathrm{~Hz}, 1 \mathrm{H}), 5.49$ (d, $J=19.0 \mathrm{~Hz}, 1 \mathrm{H}), 2.36(\mathrm{~s}, 3 \mathrm{H}), 1.29$ (s, 9H). ${ }^{13} \mathrm{C}$ NMR $\left(101 \mathrm{MHz}, \mathrm{CDCl}_{3}\right) \delta 205.3(\mathrm{~d}, J=7.0 \mathrm{~Hz}), 151.5,145.7,144.1$, $143.3,138.0(\mathrm{~d}, J=91.9 \mathrm{~Hz}), 137.0(\mathrm{~d}, J=11.1 \mathrm{~Hz}), 137.0,132.5(\mathrm{~d}, J=$ $2.8 \mathrm{~Hz}), 132.1(\mathrm{~d}, J=2.0 \mathrm{~Hz}), 132.1(\mathrm{~d}, J=10.0 \mathrm{~Hz}), 131.8(\mathrm{~d}, J=10.0$ $\mathrm{Hz}), 130.1(\mathrm{~d}, J=107.1 \mathrm{~Hz}), 129.4,128.7$ (d, $J=12.1 \mathrm{~Hz}), 128.5(\mathrm{~d}, J=$ $13.2 \mathrm{~Hz}), 128.4$ (d, $J=107.1 \mathrm{~Hz}), 128.2,128.1,128.0,127.9,127.7,127.4$, $127.3(\mathrm{~d}, J=23.2 \mathrm{~Hz}), 125.3(\mathrm{~d}, J=1.4 \mathrm{~Hz}), 125.0,120.6(\mathrm{~d}, J=7.7 \mathrm{~Hz})$, 115.4, 80.9, 34.6, 31.2, 21.6. ${ }^{31} \mathbf{P}$ NMR $\left(162 \mathrm{MHz}, \mathrm{CDCl}_{3}\right) \delta 35.85(\mathrm{~s})$. HRMS (ESI): $\left([\mathrm{M}+\mathrm{Na}]^{+}\right)$Calcd for $\mathrm{C}_{47} \mathrm{H}_{43} \mathrm{NaO}_{4} \mathrm{PS}^{+}:$757.2512, Found: 757.2504. IR (film) v 3231, 2962, 2917, 1941, 1594, 1437, 1306, 1144, $1085,833,690 \mathrm{~cm}^{-1}$.

(5-([1,1'-biphenyl]-4-yl)-3-(hydroxydiphenylmethyl)-5-tosylpenta-1,3, 4-trien-2-yl)diphenylphosphine oxide (3ea) 
(Sh

3ea

A white solid, (86 mg, 57\% yield), m.p.: 161.5 - $163.4{ }^{\circ} \mathrm{C}$. Eluent: petroleum ether/ethyl acetate=2.5:1. ${ }^{1} \mathbf{H} \mathbf{N M R}\left(400 \mathrm{MHz}, \mathrm{CDCl}_{3}\right) \delta 7.99$ $(\mathrm{d}, J=7.4 \mathrm{~Hz}, 2 \mathrm{H}), 7.91(\mathrm{~s}, 1 \mathrm{H}), 7.68-7.61(\mathrm{~m}, 2 \mathrm{H}), 7.57-7.54(\mathrm{~m}, 2 \mathrm{H})$, $7.53-7.47(\mathrm{~m}, 5 \mathrm{H}), 7.46-7.42(\mathrm{~m}, 5 \mathrm{H}), 7.41-7.35(\mathrm{~m}, 2 \mathrm{H}), 7.35-7.30$ $(\mathrm{m}, 3 \mathrm{H}), 7.26(\mathrm{~d}, J=8.3 \mathrm{~Hz}, 2 \mathrm{H}), 7.23-7.17(\mathrm{~m}, 2 \mathrm{H}), 7.17-7.05(\mathrm{~m}, 5 \mathrm{H})$, $6.67(\mathrm{~d}, J=8.3 \mathrm{~Hz}, 2 \mathrm{H}), 6.45(\mathrm{~d}, J=39.9 \mathrm{~Hz}, 1 \mathrm{H}), 5.52(\mathrm{~d}, J=19.0 \mathrm{~Hz}$, 1H), 2.37 (s, 3H). ${ }^{13} \mathrm{C}$ NMR (101 MHz, $\left.\mathrm{CDCl}_{3}\right) \delta 205.3(\mathrm{~d}, J=6.9 \mathrm{~Hz})$, 145.8, 144.3, 143.1, 141.1, 140.2, 137.9 (d, $J=92.9 \mathrm{~Hz}), 136.9,136.8$ (d, $J=11.1 \mathrm{~Hz}), 132.6(\mathrm{~d}, J=2.0 \mathrm{~Hz}), 132.3(\mathrm{~d}, J=2.0 \mathrm{~Hz}), 132.2(\mathrm{~d}, J=10.0$ Hz), 131.8 (d, $J=10.0 \mathrm{~Hz}), 130.0$ (d, $J=106.1 \mathrm{~Hz}), 129.5,128.9,128.9$, 128.8, $128.7(\mathrm{~d}, J=12.1 \mathrm{~Hz}), 128.5$ (d, $J=12.1 \mathrm{~Hz}), 128.1,128.1,127.9$, 127.7, $127.3(\mathrm{~d}, J=25.3 \mathrm{~Hz}), 127.3,126.9,126.6,120.8(\mathrm{~d}, J=7.6 \mathrm{~Hz})$, 115.4, 81.0, 21.6. ${ }^{31} \mathbf{P}$ NMR (162 $\left.\mathrm{MHz} \mathrm{CDCl}_{3}\right) \delta 35.93$ (s). HRMS (ESI): $\left([\mathrm{M}+\mathrm{Na}]^{+}\right)$Calcd for $\mathrm{C}_{49} \mathrm{H}_{39} \mathrm{NaO}_{4} \mathrm{PS}^{+}$: 777.2199, Found: 777.2191. IR (film) v 3059, 2920, 2813, 1933, 1595, 1487, 1301, 1145, 1119, 768, 703 $\mathrm{cm}^{-1}$.

(5-(4-chlorophenyl)-3-(hydroxydiphenylmethyl)-5-tosylpenta-1,3,4trien-2-yl)diphenylphosphine oxide (3fa) 


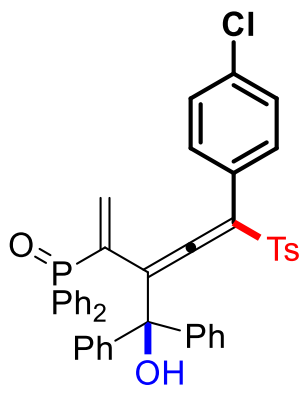

3 fa

A white solid, (97 mg, 66\% yield), m.p.: $162.4-164.4{ }^{\circ} \mathrm{C}$. Eluent: petroleum ether/ethyl acetate $=2.5: 1 .{ }^{1} \mathbf{H}$ NMR $\left(400 \mathrm{MHz}, \mathrm{CDCl}_{3}\right) \delta 7.97$ $(\mathrm{d}, J=7.5 \mathrm{~Hz}, 2 \mathrm{H}), 7.87(\mathrm{~s}, 1 \mathrm{H}), 7.67-7.59(\mathrm{~m}, 2 \mathrm{H}), 7.54-7.45(\mathrm{~m}, 4 \mathrm{H})$, $7.44-7.40(\mathrm{~m}, 3 \mathrm{H}), 7.40-7.32(\mathrm{~m}, 3 \mathrm{H}), 7.30-7.21(\mathrm{~m}, 4 \mathrm{H}), 7.15-7.03$ $(\mathrm{m}, 5 \mathrm{H}), 6.97(\mathrm{~d}, J=8.6 \mathrm{~Hz}, 2 \mathrm{H}), 6.48-6.28(\mathrm{~m}, 3 \mathrm{H}), 5.50(\mathrm{~d}, J=19.0$ $\mathrm{Hz}, 1 \mathrm{H}), 2.37(\mathrm{~s}, 3 \mathrm{H}) .{ }^{13} \mathbf{C} \mathbf{N M R}\left(101 \mathrm{MHz}, \mathrm{CDCl}_{3}\right) \delta 204.8(\mathrm{~d}, J=6.7 \mathrm{~Hz})$, 145.9, 144.5, 142.9, 137.6 (d, $J=92.9 \mathrm{~Hz}), 136.6,136.5$ (d, $J=10.0 \mathrm{~Hz})$, 134.6, $132.6(\mathrm{~d}, J=3.0 \mathrm{~Hz}), 132.4(\mathrm{~d}, J=3.0 \mathrm{~Hz}), 132.1(\mathrm{~d}, J=11.1 \mathrm{~Hz})$, $131.8(\mathrm{~d}, J=10.0 \mathrm{~Hz}), 129.9$ (d, $J=106.1 \mathrm{~Hz}), 129.8,129.5,128.7$ (d, $J=$ $13.1 \mathrm{~Hz}), 128.5(\mathrm{~d}, J=13.1 \mathrm{~Hz}), 128.2,128.1,128.0,127.8,127.7,127.6$, $127.4(\mathrm{~d}, J=31.3 \mathrm{~Hz}), 127.2,126.9(\mathrm{~d}, J=1.5 \mathrm{~Hz}), 120.7(\mathrm{~d}, J=7.3 \mathrm{~Hz})$, 114.8, 81.0, 21.7. ${ }^{31}$ P NMR (162 MHz, $\left.\mathrm{CDCl}_{3}\right) \delta 35.87$ (s). HRMS (ESI): $\left([\mathrm{M}+\mathrm{Na}]^{+}\right)$Calcd for $\mathrm{C}_{43} \mathrm{H}_{34} \mathrm{ClNaO}_{4} \mathrm{PS}^{+}:$735.1496, Found: 735.1486. IR (film) v 3209, 3059, 1953, 1594, 1490, 1442, 1316, 1303, 1147, 1086, 838, $692 \mathrm{~cm}^{-1}$.

(5-(4-fluorophenyl)-3-(hydroxydiphenylmethyl)-5-tosylpenta-1,3,4trien-2-yl)diphenylphosphine oxide (3ga) 
(sh

3ga

A white solid, (111 mg, 80\% yield), m.p.: 174.0 - $176.0{ }^{\circ} \mathrm{C}$. Eluent: petroleum ether/ethyl acetate $=2.5: 1 .{ }^{1} \mathbf{H}$ NMR $\left(400 \mathrm{MHz}, \mathrm{CDCl}_{3}\right) \delta 7.97$ $(\mathrm{d}, J=7.6 \mathrm{~Hz}, 2 \mathrm{H}), 7.83(\mathrm{~s}, 1 \mathrm{H}), 7.67-7.60(\mathrm{~m}, 2 \mathrm{H}), 7.54-7.46(\mathrm{~m}, 3 \mathrm{H})$, $7.46-7.40(\mathrm{~m}, 5 \mathrm{H}), 7.39-7.34(\mathrm{~m}, 2 \mathrm{H}), 7.30-7.22(\mathrm{~m}, 4 \mathrm{H}), 7.15-7.03$ (m, 5H), $6.75-6.66(\mathrm{~m}, 2 \mathrm{H}), 6.51-6.32(\mathrm{~m}, 3 \mathrm{H}), 5.51(\mathrm{~d}, J=19.0 \mathrm{~Hz}$, 1H), $2.36(\mathrm{~s}, 3 \mathrm{H}) .{ }^{13} \mathrm{C}$ NMR $\left(101 \mathrm{MHz}, \mathrm{CDCl}_{3}\right) \delta 204.7(\mathrm{~d}, J=6.0 \mathrm{~Hz})$, 164.0, 161.5, 145.9, 144.5, 143.0, 137.7 (d, $J=92.9 \mathrm{~Hz}), 136.6,136.5$ (d, $J=10.0 \mathrm{~Hz}), 132.6(\mathrm{~d}, J=2.7 \mathrm{~Hz}), 132.4(\mathrm{~d}, J=2.7 \mathrm{~Hz}), 132.1(\mathrm{~d}, J=10.0$ $\mathrm{Hz}), 131.8(\mathrm{~d}, J=10.0 \mathrm{~Hz}), 130.6(\mathrm{~d}, J=9.1 \mathrm{~Hz}), 130.0(\mathrm{~d}, J=106.1 \mathrm{~Hz})$, 129.5, $128.7(\mathrm{~d}, J=13.1 \mathrm{~Hz}), 128.5(\mathrm{~d}, J=2.7 \mathrm{~Hz}), 128.3$ (d, $J=107.1$ Hz), 128.1, 128.0, 127.9, 127.7, 127.3 (d, $J=32.3 \mathrm{~Hz}), 127.2,124.4$ (q, $J$ $=1.0 \mathrm{~Hz}), 120.5(\mathrm{~d}, J=7.4 \mathrm{~Hz}), 115.0(\mathrm{~d}, J=22.2 \mathrm{~Hz}), 114.8(\mathrm{~d}, J=2.0$ $\mathrm{Hz}), 81.0,21.6 .{ }^{31} \mathbf{P}$ NMR (162 MHz, $\left.\mathrm{CDCl}_{3}\right) \delta 35.78$ (s). HRMS (ESI): $\left([\mathrm{M}+\mathrm{Na}]^{+}\right)$Calcd for $\mathrm{C}_{43} \mathrm{H}_{34} \mathrm{FNaO}_{4} \mathrm{PS}^{+}:$719.1792, Found: 719.1786. IR (film) v 3181, 3061, 2929, 2789, 1945, 1594, 1437, 1303, 1143, 1084, 848, $692 \mathrm{~cm}^{-1}$.

(3-(hydroxydiphenylmethyl)-5-(m-tolyl)-5-tosylpenta-1,3,4-trien-2-yl) diphenylphosphine oxide (3ia) 


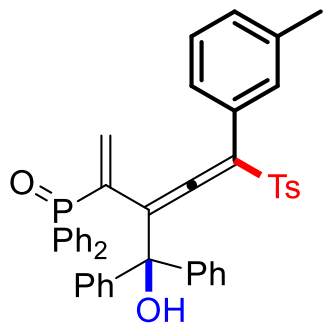

3ia

A white solid, (112 mg, 81\% yield), m.p.: 148.2 - $150.0{ }^{\circ} \mathrm{C}$. Eluent: petroleum ether/ethyl acetate $=2.5: 1 .{ }^{1} \mathbf{H}$ NMR $\left(400 \mathrm{MHz}, \mathrm{CDCl}_{3}\right) \delta 7.96$ $(\mathrm{d}, J=7.4 \mathrm{~Hz}, 2 \mathrm{H}), 7.81(\mathrm{~s}, 1 \mathrm{H}), 7.66-7.58(\mathrm{~m}, 2 \mathrm{H}), 7.54-7.46(\mathrm{~m}, 3 \mathrm{H})$, $7.44-7.41$ (m, 3H), $7.40-7.32(\mathrm{~m}, 4 \mathrm{H}), 7.28$ (d, J=8.2 Hz, 2H), 7.23 $7.16(\mathrm{~m}, 2 \mathrm{H}), 7.15-7.03(\mathrm{~m}, 5 \mathrm{H}), 7.00-6.90(\mathrm{~m}, 2 \mathrm{H}), 6.54-6.19(\mathrm{~m}$, 3H), $5.51(\mathrm{~d}, J=19.0 \mathrm{~Hz}, 1 \mathrm{H}), 2.36(\mathrm{~s}, 3 \mathrm{H}), 2.14(\mathrm{~s}, 3 \mathrm{H}) .{ }^{13} \mathbf{C}$ NMR $(101$ $\left.\mathrm{MHz}, \mathrm{CDCl}_{3}\right) \delta 205.0(\mathrm{~d}, J=7.0 \mathrm{~Hz}), 145.8,144.2,143.2,138.0(\mathrm{~d}, J=$ $91.9 \mathrm{~Hz}), 137.5,136.9$ (d, $J=10.0 \mathrm{~Hz}), 136.8,132.5$ (d, $J=2.6 \mathrm{~Hz}), 132.2$ $(\mathrm{d}, J=3.0 \mathrm{~Hz}), 132.1(\mathrm{~d}, J=10.0 \mathrm{~Hz}), 131.8(\mathrm{~d}, J=10.0 \mathrm{~Hz}), 130.0(\mathrm{~d}, J$ $=107.1 \mathrm{~Hz}), 129.4,129.3,128.7(\mathrm{~d}, J=13.1 \mathrm{~Hz}), 128.6(\mathrm{~d}, J=82.8 \mathrm{~Hz})$, $128.4(\mathrm{~d}, J=13.1 \mathrm{~Hz}), 128.1,128.0,128.0,127.7,127.7,127.3$ (d, $J=30.3$ $\mathrm{Hz}), 127.3,125.6,120.5$ (d, $J=7.7 \mathrm{~Hz}), 115.6,80.9,21.6,21.3 .{ }^{31} \mathbf{P}$ NMR $\left(162 \mathrm{MHz}, \mathrm{CDCl}_{3}\right) \delta 35.67$ (s). HRMS (ESI): $\left([\mathrm{M}+\mathrm{H}]^{+}\right)$Calcd for $\mathrm{C}_{44} \mathrm{H}_{38} \mathrm{O}_{4} \mathrm{PS}^{+}:$693.2223, Found: 693.2214. IR (film) v 3194, 3059, 2917, 1946, 1594, 1440, 1302, 1147, 1084, 1046, 723, $692 \mathrm{~cm}^{-1}$.

\section{(5-(3-fluorophenyl)-3-(hydroxydiphenylmethyl)-5-tosylpenta-1,3,4-} trien-2-yl)diphenylphosphine oxide (3ja)<smiles>C=C1C(=CC(C)(c2ccccc2)c2ccccc2)C(=O)[PH](=O)C1c1cccc(F)c1</smiles> 
A white solid, $\left(72 \mathrm{mg}, 52 \%\right.$ yield), m.p.: $157.4-159.0{ }^{\circ} \mathrm{C}$. Eluent: petroleum ether/ethyl acetate $=2.5: 1 .{ }^{1} \mathbf{H}$ NMR $\left(400 \mathrm{MHz}, \mathrm{CDCl}_{3}\right) \delta 8.02-$ $7.95(\mathrm{~m}, 2 \mathrm{H}), 7.86(\mathrm{~s}, 1 \mathrm{H}), 7.67-7.60(\mathrm{~m}, 2 \mathrm{H}), 7.54-7.51(\mathrm{~m}, 1 \mathrm{H}), 7.49$ - $7.45(\mathrm{~m}, 3 \mathrm{H}), 7.44-7.41(\mathrm{~m}, 3 \mathrm{H}), 7.40-7.32(\mathrm{~m}, 3 \mathrm{H}), 7.30-7.27$ (m, 2H), $7.26-7.21(\mathrm{~m}, 2 \mathrm{H}), 7.15-7.05(\mathrm{~m}, 5 \mathrm{H}), 7.02-6.92(\mathrm{~m}, 1 \mathrm{H}), 6.89-$ $6.81(\mathrm{~m}, 1 \mathrm{H}), 6.39(\mathrm{~d}, \mathrm{~J}=39.8 \mathrm{~Hz}, 1 \mathrm{H}), 6.31-6.23(\mathrm{~m}, 2 \mathrm{H}), 5.51(\mathrm{~d}, \mathrm{~J}=$ $19.0 \mathrm{~Hz}, 1 \mathrm{H}), 2.37(\mathrm{~s}, 3 \mathrm{H}) .{ }^{13} \mathbf{C}$ NMR $\left(101 \mathrm{MHz}, \mathrm{CDCl}_{3}\right) \delta 204.8(\mathrm{~d}, \mathrm{~J}=$ $6.7 \mathrm{~Hz}), 163.1,160.7$, 145.8, 144.5, 142.9, 137.7 (d, $J=92.9 \mathrm{~Hz}), 136.6$, 136.5, $132.6(\mathrm{~d}, \mathrm{~J}=2.6 \mathrm{~Hz}), 132.4(\mathrm{~d}, J=2.6 \mathrm{~Hz}), 132.2(\mathrm{~d}, J=10.1 \mathrm{~Hz})$, $131.8(\mathrm{~d}, \mathrm{~J}=10.0 \mathrm{~Hz}), 129.9(\mathrm{~d}, J=106.1 \mathrm{~Hz}), 129.5,129.4(\mathrm{~d}, J=8.1$ Hz), 128.7 (d, $J=13.1 \mathrm{~Hz}), 128.5(\mathrm{~d}, \mathrm{~J}=12.1 \mathrm{~Hz}), 128.1,128.0,127.9$ (d, $J=73.7 \mathrm{~Hz}), 127.9,127.7,127.4(\mathrm{~d}, J=24.2 \mathrm{~Hz}), 127.2,124.4(\mathrm{~d}, \mathrm{~J}=3.0$ Hz), 120.9 (d, J = 7.3 Hz), 115.5 (dd, J = 22.2, $10.5 \mathrm{~Hz}), 114.7$, 81.0, 21.6. ${ }^{19}$ F NMR $\left(376 \mathrm{MHz}, \mathrm{CDCl}_{3}\right) \delta-112.41$ (s). ${ }^{31} \mathbf{P}$ NMR $\left(162 \mathrm{MHz}, \mathrm{CDCl}_{3}\right)$ $\delta 35.80$ (s). HRMS (ESI): $\left([\mathrm{M}+\mathrm{H}]^{+}\right)$Calcd for $\mathrm{C}_{43} \mathrm{H}_{35} \mathrm{FO}_{4} \mathrm{PS}^{+}: 697.1972$, Found: 697.1953. IR (film) v 3193, 3054, 2917, 1948, 1593, 1432, 1300, $1147,1082,1041,722,692 \mathrm{~cm}^{-1}$.

(3-(hydroxydiphenylmethyl)-5-(o-tolyl)-5-tosylpenta-1,3,4-trien-2-yl) diphenylphosphine oxide (3ka)

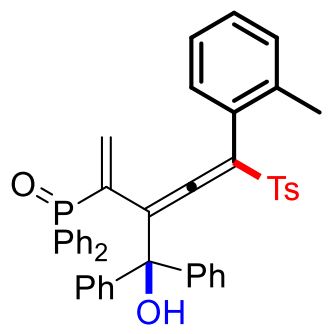

$3 \mathrm{ka}$

A white solid, (81 mg, $55 \%$ yield). Eluent: petroleum ether/ethyl acetate $=2.5: 1 .{ }^{1} \mathbf{H}$ NMR $\left(400 \mathrm{MHz}, \mathrm{CDCl}_{3}\right) \delta 7.77(\mathrm{~d}, \mathrm{~J}=7.4 \mathrm{~Hz}, 2 \mathrm{H}), 7.73$ (s, 1H), $7.59-7.52(\mathrm{~m}, 3 \mathrm{H}), 7.50-7.45(\mathrm{~m}, 1 \mathrm{H}), 7.43-7.41(\mathrm{~m}, 2 \mathrm{H}), 7.40$ 
$-7.37(\mathrm{~m}, 3 \mathrm{H}), 7.36-7.31(\mathrm{~m}, 1 \mathrm{H}), 7.28(\mathrm{~s}, 2 \mathrm{H}), 7.27-7.25(\mathrm{~m}, 2 \mathrm{H}), 7.20$ $(\mathrm{t}, \mathrm{J}=7.5 \mathrm{~Hz}, 1 \mathrm{H}), 7.15-7.10(\mathrm{~m}, 4 \mathrm{H}), 7.09-7.02(\mathrm{~m}, 4 \mathrm{H}), 6.91(\mathrm{t}, \mathrm{J}=$ $7.5 \mathrm{~Hz}, 1 \mathrm{H}), 6.69$ (d, J = 39.1 Hz, 1H), 6.07 (d, J = 7.6 Hz, 1H), 5.60 (d, J $=18.6 \mathrm{~Hz}, 1 \mathrm{H}), 2.40(\mathrm{~s}, 3 \mathrm{H}), 1.71(\mathrm{~s}, 3 \mathrm{H}) \cdot{ }^{13} \mathbf{C ~ N M R}\left(101 \mathrm{MHz}, \mathrm{CDCl}_{3}\right) \delta$ $204.4(\mathrm{~d}, \mathrm{~J}=7.9 \mathrm{~Hz}), 144.8,144.3,144.2,139.1(\mathrm{~d}, \mathrm{~J}=90.9 \mathrm{~Hz}), 138.5$, $137.9(\mathrm{~d}, \mathrm{~J}=10.9 \mathrm{~Hz}), 136.0,132.4(\mathrm{~d}, \mathrm{~J}=3.0 \mathrm{~Hz}), 132.3(\mathrm{~d}, \mathrm{~J}=3.0 \mathrm{~Hz})$, $132.0(\mathrm{~d}, \mathrm{~J}=12.1 \mathrm{~Hz}), 131.8(\mathrm{~d}, \mathrm{~J}=10.1 \mathrm{~Hz}), 131.0,130.3$ (d, J = 108.1 $\mathrm{Hz}), 130.2,130.0,129.2,128.7,128.7$ (d, J = 10.1 Hz), 128.5 (d, J = 10.1 $\mathrm{Hz}), 128.2$, 128.0, 127.7, 127.6, 127.2 (d, J = 22.2 Hz), 125.1, 121.5 (d, J $=8.9 \mathrm{~Hz}), 113.7,80.5,21.7,19.6 .{ }^{31} \mathbf{P}$ NMR $\left(162 \mathrm{MHz}, \mathrm{CDCl}_{3}\right) \delta 34.25$ (s). HRMS (ESI): $\left([\mathrm{M}+\mathrm{H}]^{+}\right)$Calcd for $\mathrm{C}_{44} \mathrm{H}_{38} \mathrm{O}_{4} \mathrm{PS}^{+}$: 693.2223, Found: 693.2220 .

(3-(hydroxydiphenylmethyl)-5-(naphthalen-1-yl)-5-tosylpenta-1,3,4trien-2-yl)diphenylphosphine oxide (3la)

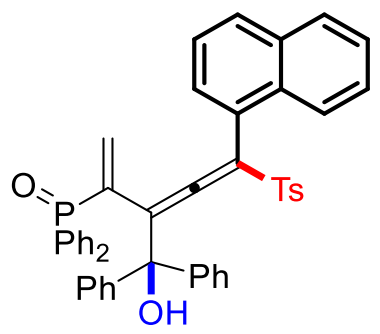

3la

A white solid, (93 mg, 62\% yield), m.p.: 149.3 - $151.1{ }^{\circ} \mathrm{C}$. Eluent: petroleum ether/ethyl acetate $=2.5: 1 .{ }^{1} \mathbf{H}$ NMR $\left(400 \mathrm{MHz}, \mathrm{CDCl}_{3}\right) \delta 8.02$ (d, $J=7.5 \mathrm{~Hz}, 2 \mathrm{H}), 7.85$ (d, $J=12.5 \mathrm{~Hz}, 1 \mathrm{H}), 7.77-7.72(\mathrm{~m}, 1 \mathrm{H}), 7.61-$ $7.52(\mathrm{~m}, 5 \mathrm{H}), 7.53-7.46(\mathrm{~m}, 6 \mathrm{H}), 7.45-7.41(\mathrm{~m}, 3 \mathrm{H}), 7.38-7.31(\mathrm{~m}$, $3 \mathrm{H}), 7.18-7.06(\mathrm{~m}, 6 \mathrm{H}), 7.04-6.95(\mathrm{~m}, 3 \mathrm{H}), 6.78-6.66(\mathrm{~m}, 1 \mathrm{H}), 6.44$ $(\mathrm{d}, J=39.8 \mathrm{~Hz}, 1 \mathrm{H}), 5.53(\mathrm{~d}, J=19.0 \mathrm{~Hz}, 1 \mathrm{H}), 2.33(\mathrm{~s}, 3 \mathrm{H}) .{ }^{13} \mathbf{C} \mathbf{N M R}$ $\left(101 \mathrm{MHz}, \mathrm{CDCl}_{3}\right) \delta 205.5(\mathrm{~d}, J=6.9 \mathrm{~Hz}), 146.1,144.3,143.2,137.9(\mathrm{~d}$, $J=92.9 \mathrm{~Hz}), 136.9,136.5(\mathrm{~d}, J=9.9 \mathrm{~Hz}), 132.8(\mathrm{~d}, J=24.2 \mathrm{~Hz}), 132.6(\mathrm{~d}$, $J=3.0 \mathrm{~Hz}), 132.2$ (d, $J=9.9 \mathrm{~Hz}), 132.1$ (d, $J=5.1 \mathrm{~Hz}), 131.7$ (d, $J=9.9$ 
Hz), 129.9 (d, $J=106.1 \mathrm{~Hz}), 129.4,128.7$ (d, $J=13.1 \mathrm{~Hz}), 128.4(\mathrm{~d}, J=$ $1.0 \mathrm{~Hz}), 128.3,128.2(\mathrm{~d}, J=107.1 \mathrm{~Hz}), 128.1,128.1,127.9,127.8,127.5$ (d, $J=8.1 \mathrm{~Hz}), 127.3(\mathrm{~d}, J=20.2 \mathrm{~Hz}), 127.3,126.7,126.0(\mathrm{~d}, J=32.3 \mathrm{~Hz})$, $125.8(\mathrm{~d}, J=2.0 \mathrm{~Hz}), 120.6(\mathrm{~d}, J=7.7 \mathrm{~Hz}), 115.9,100.0,81.0,21.6 .{ }^{31} \mathbf{P}$ NMR (162 MHz, $\left.\mathrm{CDCl}_{3}\right) \delta 35.77$ (s). HRMS (ESI): ([M+H] $\left.]^{+}\right)$Calcd for $\mathrm{C}_{47} \mathrm{H}_{38} \mathrm{O}_{4} \mathrm{PS}^{+}:$729.2223, Found: 729.2219. IR (film) $v$ 3054, 2917, 2791, $1922,1594,1435,1303,1156,1117,964,818,689 \mathrm{~cm}^{-1}$.

(3-(hydroxydiphenylmethyl)-5-(pyridin-3-yl)-5-tosylpenta-1,3,4-trien2-yl)diphenylphosphine oxide (3ma)

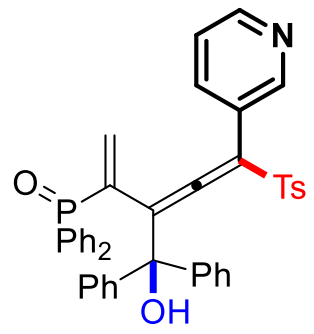

$3 \mathrm{ma}$

A white solid, (84 mg, 64\% yield), m.p.: $141.2-143.0{ }^{\circ} \mathrm{C}$. Eluent: petroleum ether/ethyl acetate=1:2. ${ }^{1} \mathbf{H}$ NMR $\left(400 \mathrm{MHz}, \mathrm{CDCl}_{3}\right) \delta 8.44-$ $8.34(\mathrm{~m}, 1 \mathrm{H}), 7.98(\mathrm{~d}, J=7.4 \mathrm{~Hz}, 2 \mathrm{H}), 7.82(\mathrm{~s}, 1 \mathrm{H}), 7.65-7.60(\mathrm{~m}, 2 \mathrm{H})$, $7.55-7.51(\mathrm{~m}, 2 \mathrm{H}), 7.51-7.46(\mathrm{~m}, 3 \mathrm{H}), 7.45-7.41(\mathrm{~m}, 2 \mathrm{H}), 7.40-7.34$ (m, 4H), $7.29-7.21(\mathrm{~m}, 4 \mathrm{H}), 7.17-7.06(\mathrm{~m}, 5 \mathrm{H}), 6.99-6.91(\mathrm{~m}, 1 \mathrm{H})$, $6.85-6.78(\mathrm{~m}, 1 \mathrm{H}), 6.37(\mathrm{~d}, J=39.9 \mathrm{~Hz}, 1 \mathrm{H}), 5.52(\mathrm{~d}, J=19.0 \mathrm{~Hz}, 1 \mathrm{H})$, $2.36(\mathrm{~s}, 3 \mathrm{H}) .{ }^{13} \mathbf{C}$ NMR $\left(101 \mathrm{MHz}, \mathrm{CDCl}_{3}\right) \delta 204.6(\mathrm{~d}, J=6.7 \mathrm{~Hz}), 149.4$, 149.0, 145.8 (d, $J=17.2 \mathrm{~Hz}), 142.7,137.4$ (d, $J=92.9 \mathrm{~Hz}), 136.3$ (d, $J=$ $10.0 \mathrm{~Hz}), 136.1$ (d, $J=39.4 \mathrm{~Hz}), 132.7$ (d, $J=3.0 \mathrm{~Hz}), 132.5$ (d, $J=3.0$ $\mathrm{Hz}), 132.1$ (d, $J=10.2 \mathrm{~Hz}), 131.8(\mathrm{~d}, J=10.0 \mathrm{~Hz}), 130.1,129.7(\mathrm{~d}, J=$ $106.1 \mathrm{~Hz}), 129.6,128.7(\mathrm{~d}, J=12.1 \mathrm{~Hz}), 128.5(\mathrm{~d}, J=12.1 \mathrm{~Hz}), 128.3$, $128.1,128.1(\mathrm{~d}, J=106.1 \mathrm{~Hz}), 128.0,127.9,127.8,127.5$ (d, $J=24.2 \mathrm{~Hz})$, $127.1,125.1,122.7,121.0(\mathrm{~d}, J=7.2 \mathrm{~Hz}), 113.0,81.0,21.6 .{ }^{31} \mathbf{P}$ NMR $(162$ 
$\left.\mathrm{MHz}, \mathrm{CDCl}_{3}\right) \quad \delta \quad 35.80$ (s). HRMS (ESI): $\left([\mathrm{M}+\mathrm{H}]^{+}\right)$Calcd for $\mathrm{C}_{42} \mathrm{H}_{35} \mathrm{NO}_{4} \mathrm{PS}^{+}:$680.2019, Found: 680.2018. IR (film) v 3553, 2988, 2901, 1952, 1594, 1438, 1315, 1148, 1085, 812, 728, $693 \mathrm{~cm}^{-1}$.

\section{(3-(hydroxydiphenylmethyl)-5-(thiophen-3-yl)-5-tosylpenta-1,3,4-} trien-2-yl)diphenylphosphine oxide (3na)

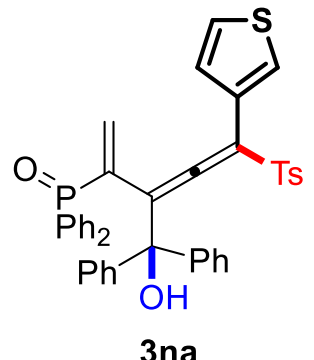

A white solid, (79 mg, 58\% yield), m.p.: 146.2 - $148.0{ }^{\circ} \mathrm{C}$. Eluent: petroleum ether/ethyl acetate $=1: 2 .{ }^{1} \mathbf{H}$ NMR $\left(600 \mathrm{MHz}, \mathrm{CDCl}_{3}\right) \delta 7.94(\mathrm{~d}$, $\mathrm{J}=7.3 \mathrm{~Hz}, 2 \mathrm{H}), 7.85$ (s, 1H), $7.65-7.60$ (m, 2H), $7.53-7.47$ (m, 2H), $7.47-7.41(\mathrm{~m}, 3 \mathrm{H}), 7.41-7.37(\mathrm{~m}, 2 \mathrm{H}), 7.36-7.30(\mathrm{~m}, 4 \mathrm{H}), 7.27$ (d, J = $8.3 \mathrm{~Hz}, 2 \mathrm{H}), 7.22-7.07(\mathrm{~m}, 2 \mathrm{H}), 7.10(\mathrm{~d}, \mathrm{~J}=8.2 \mathrm{~Hz}, 2 \mathrm{H}), 7.07-7.03(\mathrm{~m}$, 1H), $7.02-6.97(\mathrm{~m}, 2 \mathrm{H}), 6.91(\mathrm{dd}, \mathrm{J}=5.0,3.0 \mathrm{~Hz}, 1 \mathrm{H}), 6.77(\mathrm{dd}, \mathrm{J}=2.9$, $1.1 \mathrm{~Hz}, 1 \mathrm{H}), 6.38-6.29(\mathrm{~m}, 2 \mathrm{H}), 5.44(\mathrm{~d}, \mathrm{~J}=19.1 \mathrm{~Hz}, 1 \mathrm{H}), 2.34(\mathrm{~s}, 3 \mathrm{H})$. ${ }^{13}$ C NMR $\left(151 \mathrm{MHz}, \mathrm{CDCl}_{3}\right) \delta 204.9(\mathrm{~d}, \mathrm{~J}=6.7 \mathrm{~Hz}), 146.0,144.3,142.9$, $137.6(\mathrm{~d}, \mathrm{~J}=92.1 \mathrm{~Hz}), 137.0,136.5(\mathrm{~d}, \mathrm{~J}=10.2 \mathrm{~Hz}), 132.6(\mathrm{~d}, \mathrm{~J}=2.7 \mathrm{~Hz})$, $132.3(\mathrm{~d}, \mathrm{~J}=2.7 \mathrm{~Hz}), 132.2(\mathrm{~d}, \mathrm{~J}=10.6 \mathrm{~Hz}), 131.8(\mathrm{~d}, \mathrm{~J}=10.0 \mathrm{~Hz}), 130.2$ $(\mathrm{d}, \mathrm{J}=18.8 \mathrm{~Hz}), 129.5,128.7(\mathrm{~d}, \mathrm{~J}=12.3 \mathrm{~Hz}), 128.4(\mathrm{~d}, \mathrm{~J}=13.6 \mathrm{~Hz}), 128.1$, $128.1, \quad 128.0(\mathrm{~d}, \mathrm{~J}=84.6 \mathrm{~Hz}), 127.6,127.6,127.6,127.6,127.3(\mathrm{~d}, \mathrm{~J}=$ $40.8 \mathrm{~Hz}), 127.1,124.8$ (d, J = 3.8 Hz), 120.7 (d, J = 7.5 Hz), 112.1, 81.1, 21.6. ${ }^{31}$ P NMR (243 MHz, $\left.\mathrm{CDCl}_{3}\right) \delta 36.37$ (s). HRMS (ESI): $\left([\mathrm{M}+\mathrm{H}]^{+}\right)$ Calcd for $\mathrm{C}_{41} \mathrm{H}_{34} \mathrm{O}_{4} \mathrm{PS}_{2}^{+}$: 685.1631, Found: 685.1617. IR (film) v 3198, 3061, 2924, 1942, 1574, 1392, 1313, 1143, 951, 761, $693 \mathrm{~cm}^{-1}$. 
(3-(hydroxydiphenylmethyl)-5-tosylpenta-1,3,4-trien-2-yl)diphenylphosphine oxide (3oa)<smiles>C=C1C(=O)[PH](=O)C(O)(c2ccccc2)C(=CC(C)C)C1(O)c1ccccc1</smiles>

A white solid, (81 mg, 55\% yield). Eluent: petroleum ether/ethyl acetate $=2.5: 1 .{ }^{1} \mathbf{H}$ NMR $\left(400 \mathrm{MHz}, \mathrm{CDCl}_{3}\right) \delta 7.77-7.68(\mathrm{~m}, 4 \mathrm{H}), 7.66(\mathrm{~s}$, 1H), $7.61-7.54(\mathrm{~m}, 2 \mathrm{H}), 7.54-7.50(\mathrm{~m}, 2 \mathrm{H}), 7.49-7.43(\mathrm{~m}, 4 \mathrm{H}), 7.40-$ $7.34(\mathrm{~m}, 4 \mathrm{H}), 7.33-7.28(\mathrm{~m}, 1 \mathrm{H}), 7.27-7.19(\mathrm{~m}, 4 \mathrm{H}), 7.08-7.05(\mathrm{~m}$, 2H), $6.24(\mathrm{~d}, \mathrm{~J}=39.6 \mathrm{~Hz}, 1 \mathrm{H}), 5.63(\mathrm{~d}, \mathrm{~J}=4.4 \mathrm{~Hz}, 1 \mathrm{H}), 5.46(\mathrm{~d}, \mathrm{~J}=18.9$ $\mathrm{Hz}, 1 \mathrm{H}), 2.42(\mathrm{~s}, 3 \mathrm{H}) .{ }^{13} \mathbf{C} \mathbf{N M R}\left(101 \mathrm{MHz}, \mathrm{CDCl}_{3}\right) \delta 204.9(\mathrm{~d}, \mathrm{~J}=7.0 \mathrm{~Hz})$, 145. 1, 144.4, 142.6, 137.9, 137.1 (d, J = 71.7 Hz), $136.5(\mathrm{~d}, \mathrm{~J}=11.1 \mathrm{~Hz})$, 132.7 (d, J = 2.0 Hz), 132.6 (d, J = 2.0 Hz), 132.1, 132.0, 129.7, 129.6 (d, $\mathrm{J}=106.1 \mathrm{~Hz}), 128.9(\mathrm{~d}, \mathrm{~J}=106.1 \mathrm{~Hz}), 128.8(\mathrm{~d}, \mathrm{~J}=2.0 \mathrm{~Hz}), 128.7$ (d, J = $2.0 \mathrm{~Hz}), 128.0,128.0,127.5,127.4,127.3,127.3(\mathrm{~d}, \mathrm{~J}=22.2 \mathrm{~Hz}), 121.4(\mathrm{~d}$, $\mathrm{J}=7.7 \mathrm{~Hz}), 101.2,80.7,21.7 .{ }^{31} \mathbf{P}$ NMR (162 MHz, $\left.\mathrm{CDCl}_{3}\right) \delta 35.39$ (s). HRMS (ESI): $\left([\mathrm{M}+\mathrm{H}]^{+}\right)$Calcd for $\mathrm{C}_{37} \mathrm{H}_{32} \mathrm{O}_{4} \mathrm{PS}^{+}$: 603.1753, Found: 603.1752 .

(3-(hydroxydiphenylmethyl)-5-tosylundeca-1,3,4-trien-2-yl)diphenyl phosphine oxide (3pa)

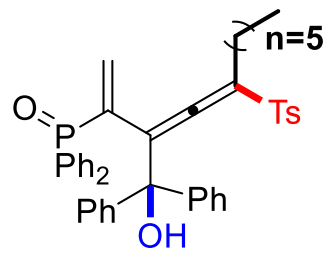

3 pa

A white solid, (124 mg, 91\% yield), m.p.: 155.8 - $157.7{ }^{\circ} \mathrm{C}$. Eluent: petroleum ether/ethyl acetate=2.5:1. ${ }^{1} \mathbf{H}$ NMR $\left(400 \mathrm{MHz}, \mathrm{CDCl}_{3}\right) \delta 7.95$ 
$(\mathrm{d}, J=7.5 \mathrm{~Hz}, 2 \mathrm{H}), 7.83(\mathrm{~s}, 1 \mathrm{H}), 7.80-7.71(\mathrm{~m}, 2 \mathrm{H}), 7.58-7.49(\mathrm{~m}, 2 \mathrm{H})$, $7.46-7.42$ (m, 6H), $7.41-7.37$ (m, 5H), $7.34-7.27$ (m, 1H), 7.24 (d, $J=$ $8.1 \mathrm{~Hz}, 2 \mathrm{H}), 7.14-7.07$ (m, 3H), 6.30 (d, $J=40.8 \mathrm{~Hz}, 1 \mathrm{H}), 5.42$ (d, $J=$ $19.2 \mathrm{~Hz}, 1 \mathrm{H}), 2.43$ (s, 3H), $1.46-1.28$ (m, 2H), $1.18-1.09$ (m, 2H), 1.00 $-0.91(\mathrm{~m}, 2 \mathrm{H}), 0.85$ (t, $J=7.3 \mathrm{~Hz}, 3 \mathrm{H}), 0.83-0.73(\mathrm{~m}, 2 \mathrm{H}), 0.54-0.36$ (m, 2H). ${ }^{13} \mathbf{C ~ N M R}\left(101 \mathrm{MHz}, \mathrm{CDCl}_{3}\right) \delta 203.4(\mathrm{~d}, J=6.7 \mathrm{~Hz}), 146.0,144.3$, $142.9,137.3$ (d, $J=92.9 \mathrm{~Hz}), 136.9,136.1$ (d, $J=10.6 \mathrm{~Hz}), 132.5$ (d, $J=$ $4.5 \mathrm{~Hz}), 132.5(\mathrm{~d}, J=2.7 \mathrm{~Hz}), 132.1,132.0,130.4$ (d, $J=106.1 \mathrm{~Hz}), 129.6$, $129.0(\mathrm{~d}, J=106.1 \mathrm{~Hz}), 128.7(\mathrm{~d}, J=3.0 \mathrm{~Hz}), 128.6(\mathrm{~d}, J=3.0 \mathrm{~Hz}), 128.1$, 127.9, 127.4, 127.3, $127.2(\mathrm{~d}, J=32.3 \mathrm{~Hz}), 120.3(\mathrm{~d}, J=7.1 \mathrm{~Hz}), 114.7$, 80.8, 31.2, 28.9, 27.5, 26.7, 22.4, 21.7, 14.1. ${ }^{31} \mathbf{P}$ NMR (162 MHz, $\left.\mathrm{CDCl}_{3}\right)$ $\delta 36.38$ (s). HRMS (ESI): ([M+H] $\left.]^{+}\right)$Calcd for $\mathrm{C}_{43} \mathrm{H}_{44} \mathrm{O}_{4} \mathrm{PS}^{+}: 687.2692$, Found: 687.2687. IR (film) v 3055, 2920, 2856, 2795, 1922, 1595, 1435, $1310,1142,1083,818,774,695 \mathrm{~cm}^{-1}$.

\section{(3-(hydroxydi-p-tolylmethyl)-5-phenyl-5-tosylpenta-1,3,4-trien-2-yl)}

\section{diphenylphosphine oxide (3qa)}

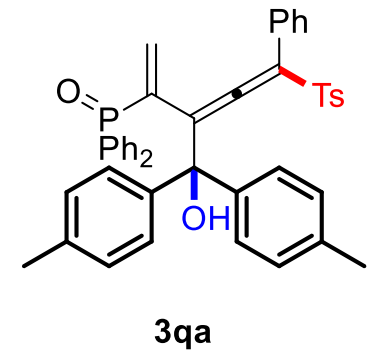

A white solid, (92 mg, 65\% yield), m.p.: 160.2 - $161.9{ }^{\circ} \mathrm{C}$. Eluent: petroleum ether/ethyl acetate=2.5:1. ${ }^{1} \mathbf{H}$ NMR $\left(400 \mathrm{MHz}, \mathrm{CDCl}_{3}\right) \delta 7.79$ $(\mathrm{d}, J=8.1 \mathrm{~Hz}, 2 \mathrm{H}), 7.65-7.57(\mathrm{~m}, 3 \mathrm{H}), 7.56-7.45(\mathrm{~m}, 3 \mathrm{H}), 7.44-7.35$ $(\mathrm{m}, 3 \mathrm{H}), 7.23(\mathrm{~d}, J=8.1 \mathrm{~Hz}, 6 \mathrm{H}), 7.20-7.16(\mathrm{~m}, 2 \mathrm{H}), 7.12-7.00(\mathrm{~m}, 4 \mathrm{H})$, $6.85(\mathrm{~d}, J=8.0 \mathrm{~Hz}, 2 \mathrm{H}), 6.61(\mathrm{~d}, J=7.5 \mathrm{~Hz}, 2 \mathrm{H}), 6.44(\mathrm{~d}, J=40.8 \mathrm{~Hz}, 1 \mathrm{H})$, $5.52(\mathrm{~d}, J=17.0 \mathrm{~Hz}, 1 \mathrm{H}), 2.40$ (s, 3H), 2.37 (s, 3H), 2.21 (s, 3H). ${ }^{13} \mathbf{C}$ NMR 
$\left(101 \mathrm{MHz}, \mathrm{CDCl}_{3}\right) \delta 205.1(\mathrm{~d}, J=7.0 \mathrm{~Hz}), 144.1,142.9,140.3,138.0(\mathrm{~d}$, $J=91.9 \mathrm{~Hz}), 136.8(\mathrm{~d}, J=3.0 \mathrm{~Hz}), 136.5,132.4(\mathrm{~d}, J=2.7 \mathrm{~Hz}), 132.2(\mathrm{~d}$, $J=2.7 \mathrm{~Hz}), 132.1(\mathrm{~d}, J=12.1 \mathrm{~Hz}), 131.8(\mathrm{~d}, J=10.0 \mathrm{~Hz}), 130.1(\mathrm{~d}, J=$ $106.1 \mathrm{~Hz}), 129.3,128.8,128.7,128.6(\mathrm{~d}, J=106.1 \mathrm{~Hz}), 128.6(\mathrm{~d}, J=13.1$ $\mathrm{Hz}), 128.5,128.4$ (d, $J=12.1 \mathrm{~Hz}), 128.3,128.0,128.0,127.9,127.2,121.2$ (d, $J=7.9 \mathrm{~Hz}), 115.2,80.7,21.6,21.2,21.0 .{ }^{31} \mathbf{P}$ NMR (162 MHz, $\left.\mathrm{CDCl}_{3}\right)$ $\delta 35.42$ (s). HRMS (ESI): ([M+Na] $\left.]^{+}\right)$Calcd for $\mathrm{C}_{45} \mathrm{H}_{39} \mathrm{NaO}_{4} \mathrm{PS}^{+}$: 729.2199 , Found: 729.2197. IR (film) v 3131, 3064, 2924, 2775, 1947, 1593, 1438, $1301,1140,1070,813,692 \mathrm{~cm}^{-1}$.

(3-(bis(4-chlorophenyl)(hydroxy)methyl)-5-phenyl-5-tosylpenta-1,3,4trien-2-yl)diphenylphosphine oxide (3ra)

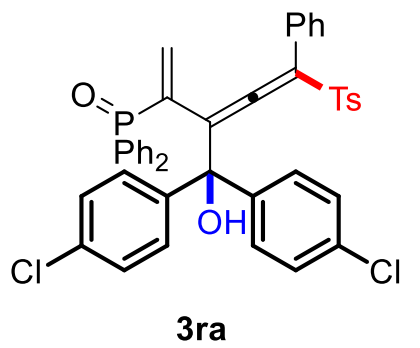

A white solid, (94 mg, 63\% yield), m.p.: 163.5 - $165.3{ }^{\circ} \mathrm{C}$. Eluent: petroleum ether/ethyl acetate $=2.5: 1 .{ }^{1} \mathbf{H}$ NMR $\left(400 \mathrm{MHz}, \mathrm{CDCl}_{3}\right) \delta 8.06$ (s, 1H), $7.81(\mathrm{~d}, J=8.6 \mathrm{~Hz}, 2 \mathrm{H}), 7.63-7.55(\mathrm{~m}, 3 \mathrm{H}), 7.51-7.43(\mathrm{~m}, 4 \mathrm{H})$, $7.43-7.37$ (m, 3H), $7.30-7.27$ (m, 2H), $7.25-7.19$ (m, 3H), 7.17 (d, $J=$ $2.8 \mathrm{~Hz}, 3 \mathrm{H}), 7.15-7.07(\mathrm{~m}, 2 \mathrm{H}), 7.02(\mathrm{~d}, J=8.6 \mathrm{~Hz}, 2 \mathrm{H}), 6.66(\mathrm{~d}, J=7.3$ Hz, 2H), 6.48 (d, $J=39.4 \mathrm{~Hz}, 1 \mathrm{H}), 5.52$ (d, $J=18.8 \mathrm{~Hz}, 1 \mathrm{H}), 2.39$ (s, 3H). ${ }^{13}$ C NMR $\left(101 \mathrm{MHz}, \mathrm{CDCl}_{3}\right) \delta 205.1(\mathrm{~d}, J=7.3 \mathrm{~Hz}), 144.6,143.6,141.5$, $138.0(\mathrm{~d}, J=76.8 \mathrm{~Hz}), 137.5(\mathrm{~d}, J=5.0 \mathrm{~Hz}), 136.4,133.4(\mathrm{~d}, J=24.2 \mathrm{~Hz})$, $132.7(\mathrm{~d}, J=2.4 \mathrm{~Hz}), 132.4(\mathrm{~d}, J=2.7 \mathrm{~Hz}), 132.0(\mathrm{~d}, J=11.1 \mathrm{~Hz}), 131.7$ $(\mathrm{d}, J=10.0 \mathrm{~Hz}), 131.1(\mathrm{~d}, J=53.5 \mathrm{~Hz}), 129.5(\mathrm{~d}, J=108.1 \mathrm{~Hz}), 129.5$, $129.5,128.9,128.7$ (d, $J=3.0 \mathrm{~Hz}), 128.6,128.5,128.2$, 128.2, 127.8, 127.8 , 
$120.8(\mathrm{~d}, J=8.1 \mathrm{~Hz}), 115.7,80.0,21.6 .{ }^{31} \mathbf{P}$ NMR $\left(162 \mathrm{MHz}, \mathrm{CDCl}_{3}\right) \delta$ 35.72 (s). HRMS (ESI): $\left([\mathrm{M}+\mathrm{H}]^{+}\right)$Calcd for $\mathrm{C}_{43} \mathrm{H}_{34} \mathrm{Cl}_{2} \mathrm{O}_{4} \mathrm{PS}^{+}$: 747.1287, Found: 747.1284. IR (film) v 3057, 2960, 2922, 2850, 1941, 1592, 1488, $1436,1312,1145,1086,1013,903,727,691 \mathrm{~cm}^{-1}$.

(3-(bis(4-fluorophenyl)(hydroxy)methyl)-5-phenyl-5-tosylpenta-1,3,4trien-2-yl)diphenylphosphine oxide (3sa)

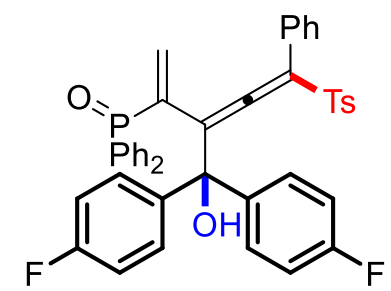

3sa

A white solid, (89 mg, 62\% yield), m.p.: $154.1-156.0{ }^{\circ} \mathrm{C}$. Eluent: petroleum ether/ethyl acetate=2.5:1. ${ }^{1} \mathbf{H}$ NMR $\left(400 \mathrm{MHz}, \mathrm{CDCl}_{3}\right) \delta 7.99$ (s, 1H), $7.93-7.85(\mathrm{~m}, 2 \mathrm{H}), 7.64-7.54(\mathrm{~m}, 3 \mathrm{H}), 7.50-7.37$ (m, 5H), 7.34 - $7.28(\mathrm{~m}, 2 \mathrm{H}), 7.25-7.17(\mathrm{~m}, 5 \mathrm{H}), 7.16-7.03(\mathrm{~m}, 6 \mathrm{H}), 6.80-6.70(\mathrm{~m}$, 2H), $6.64(\mathrm{~d}, J=7.5 \mathrm{~Hz}, 2 \mathrm{H}), 6.44(\mathrm{~d}, J=39.5 \mathrm{~Hz}, 1 \mathrm{H}), 5.51(\mathrm{~d}, J=18.9$ $\mathrm{Hz}, 1 \mathrm{H}), 2.37(\mathrm{~s}, 3 \mathrm{H}) .{ }^{13} \mathbf{C} \mathbf{N M R}\left(101 \mathrm{MHz}, \mathrm{CDCl}_{3}\right) \delta 205.0(\mathrm{~d}, J=7.3 \mathrm{~Hz})$, $163.3(\mathrm{~d}, J=24.2 \mathrm{~Hz}), 160.9(\mathrm{~d}, J=24.2 \mathrm{~Hz}), 144.6,141.3(\mathrm{~d}, J=2.5 \mathrm{~Hz})$, $138.9(\mathrm{~d}, J=3.0 \mathrm{~Hz}), 138.0(\mathrm{~d}, J=91.9 \mathrm{~Hz}), 137.4,136.5,132.7$ (d, $J=$ $3.0 \mathrm{~Hz}), 132.4(\mathrm{~d}, J=3.0 \mathrm{~Hz}), 132.0$ (d, $J=10.0 \mathrm{~Hz}), 131.8$ (d, $J=10.0$ Hz), 129.8 (d, $J=7.1 \mathrm{~Hz}), 129.7$ (d, $J=105.0 \mathrm{~Hz}), 129.4,129.2$ (d, $J=8.1$ Hz), 128.8 (d, $J=7.1 \mathrm{~Hz}), 128.7$ (d, $J=5.1 \mathrm{~Hz}), 128.5,128.4$ (d, $J=107.1$ Hz), 128.2, 128.0 (d, $J=40.4 \mathrm{~Hz}), 127.8,121.1$ (d, $J=8.4 \mathrm{~Hz}), 115.7$, $114.9(\mathrm{~d}, J=21.2 \mathrm{~Hz}), 114.3(\mathrm{~d}, J=21.2 \mathrm{~Hz}), 80.1,21.6 .{ }^{31} \mathbf{P}$ NMR $(162$ $\left.\mathrm{MHz}, \mathrm{CDCl}_{3}\right) \quad \delta \quad 35.79$ (s). HRMS (ESI): ([M+H] $\left.]^{+}\right)$Calcd for $\mathrm{C}_{43} \mathrm{H}_{34} \mathrm{~F}_{2} \mathrm{O}_{4} \mathrm{PS}^{+}$: 715.1878, Found: 715.1871. IR (film) v 3173, 3062, 2920, 2786, 1945, 1595, 1505, 1437, 1303, 1142, 1119, 828, $691 \mathrm{~cm}^{-1}$. 
(5-((4-bromophenyl)sulfonyl)-3-(1-hydroxy-1-phenylethyl)-5-phenyl penta-1,3,4-trien-2-yl)diphenylphosphine oxide (3ua)<smiles>C=C(C(=CC(=CC(=O)[PH](=O)c1ccccc1)c1ccc(Br)cc1)c1ccccc1)C(C)(O)P</smiles>

3ua

A white solid, (37 mg, 27\% yield), m.p.: $137.1-139.0{ }^{\circ} \mathrm{C}$. Eluent: petroleum ether/ethyl acetate $=2.5: 1 .{ }^{1} \mathbf{H}$ NMR $\left(600 \mathrm{MHz}, \mathrm{CDCl}_{3}\right) \delta 7.70$ $(\mathrm{d}, \mathrm{J}=7.4 \mathrm{~Hz}, 2 \mathrm{H}), 7.60-7.56(\mathrm{~m}, 4 \mathrm{H}), 7.55(\mathrm{~d}, \mathrm{~J}=8.7 \mathrm{~Hz}, 2 \mathrm{H}), 7.51-$ 7.47 (m, 2H), $7.43-7.39$ (m, 3H), 7.37 - 7.34 (m, 3H), 7.33 (d, J = 7.2 Hz, 1H), $7.29-7.26(\mathrm{~m}, 2 \mathrm{H}), 7.25-7.23(\mathrm{~m}, 1 \mathrm{H}), 7.14(\mathrm{~d}, \mathrm{~J}=4.3 \mathrm{~Hz}, 4 \mathrm{H})$, $5.98(\mathrm{~d}, \mathrm{~J}=39.8 \mathrm{~Hz}, 1 \mathrm{H}), 5.27(\mathrm{~d}, \mathrm{~J}=18.9 \mathrm{~Hz}, 1 \mathrm{H}), 1.72(\mathrm{~s}, 3 \mathrm{H}) .{ }^{13} \mathbf{C}$ NMR $\left(101 \mathrm{MHz}, \mathrm{CDCl}_{3}\right) \delta 204.3(\mathrm{~d}, \mathrm{~J}=6.9 \mathrm{~Hz}), 145.3,139.2,137.5(\mathrm{~d}, \mathrm{~J}=92.9$ Hz), $136.6(\mathrm{~d}, \mathrm{~J}=11.1 \mathrm{~Hz}), 132.6(\mathrm{~d}, \mathrm{~J}=2.6 \mathrm{~Hz}), 132.4,132.3,132.1$ (d, J = 10.1 Hz), 131.7 (d, J = 42.4 Hz), 131.7 (d, J = 10.1 Hz), 129.6 (d, J = 107.1 Hz), 129.4, 128.9, $128.6(\mathrm{~d}, \mathrm{~J}=17.2 \mathrm{~Hz}), 128.6,128.4,128.2,128.0$ $(\mathrm{d}, \mathrm{J}=13.1 \mathrm{~Hz}), 127.4,127.0,126.1,120.2(\mathrm{~d}, \mathrm{~J}=8.0 \mathrm{~Hz}), 115.6,76.0$, 30.8. ${ }^{31}$ P NMR $\left(162 \mathrm{MHz}, \mathrm{CDCl}_{3}\right) \delta 35.31$ (s). HRMS (ESI): $\left([\mathrm{M}+\mathrm{H}]^{+}\right)$ Calcd for $\mathrm{C}_{37} \mathrm{H}_{31} \mathrm{BrO}_{4} \mathrm{PS}^{+}$: 681.0859, Found: 681.0865. IR (film) v 3228, $3058,1932,1572,1433,1392,1315,1173,955,822,693 \mathrm{~cm}^{-1}$.

(1-(3-hydroxy-1,4-diphenyl-7-tosylnaphthalen-2-yl)ethyl)diphenyl phosphine oxide (4) 


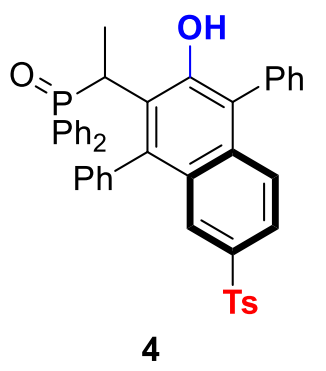

A white solid (68 mg, 50\% yield), m.p.: 321.6 - $322.8{ }^{\circ} \mathrm{C}$. Eluent: petroleum ether/ethyl acetate $=3: 1 .{ }^{1} \mathbf{H} \mathbf{~ N M R}\left(600 \mathrm{MHz}, \mathrm{CDCl}_{3}\right) \delta 11.22(\mathrm{~s}$, 1H), $7.71(\mathrm{~s}, 1 \mathrm{H}), 7.64(\mathrm{~d}, \mathrm{~J}=8.3 \mathrm{~Hz}, 2 \mathrm{H}), 7.60-7.55(\mathrm{~m}, 3 \mathrm{H}), 7.54(\mathrm{~s}$, 4H), $7.53-7.48(\mathrm{~m}, 5 \mathrm{H}), 7.48-7.43(\mathrm{~m}, 3 \mathrm{H}), 7.36(\mathrm{~d}, \mathrm{~J}=7.5 \mathrm{~Hz}, 1 \mathrm{H})$, $7.34-7.29(\mathrm{~m}, 5 \mathrm{H}), 7.23-7.18(\mathrm{~m}, 3 \mathrm{H}), 3.81-3.73(\mathrm{~m}, 1 \mathrm{H}), 2.34(\mathrm{~s}, 3 \mathrm{H})$, $1.57-1.52(\mathrm{~m}, 3 \mathrm{H}) .{ }^{13} \mathrm{C}$ NMR $\left(151 \mathrm{MHz}, \mathrm{CDCl}_{3}\right) \delta 153.9,143.7,142.1$ $(\mathrm{d}, \mathrm{J}=7.6 \mathrm{~Hz}), 138.9,137.8,136.3,135.5(\mathrm{~d}, \mathrm{~J}=95.1 \mathrm{~Hz}), 132.5,132.4$ (d, $\mathrm{J}=1.5 \mathrm{~Hz}), 131.2,131.2,131.2(\mathrm{~d}, \mathrm{~J}=7.6 \mathrm{~Hz}), 130.6(\mathrm{~d}, \mathrm{~J}=80.0 \mathrm{~Hz})$, $130.5(\mathrm{~d}, \mathrm{~J}=101.2 \mathrm{~Hz}), 129.7,129,1$ (d, J = 12.1 Hz), 128.9 (d, J = 25.7 Hz), 128.4 (d, J = 39.3 Hz), 128.4 (d, J = 12.1 Hz), 128.4, 128.0, 127.8 (d, $\mathrm{J}=5.2 \mathrm{~Hz}), 127.6,127.3,126.6,126.6(\mathrm{~d}, \mathrm{~J}=66.4 \mathrm{~Hz}), 122.4,38.2(\mathrm{~d}, \mathrm{~J}=$ $66.4 \mathrm{~Hz}), 21.5,13.2 .{ }^{31} \mathbf{P}$ NMR (243 MHz, $\left.\mathrm{CDCl}_{3}\right) \delta 42.15$ (s). HRMS (ESI): $\left([\mathrm{M}+\mathrm{H}]^{+}\right)$Calcd for C43H36O4PS ${ }^{+}: 679.2066$, Found: 679.2069.

(4-chloro-3-(diphenylmethylene)-5-phenyl-5-tosylpenta-1,4-dien-2yl)diphenylphosphine oxide (5a)<smiles>C=C(P=O)C(C(Cl)=C(P)[As])=C(c1ccccc1)c1ccccc1</smiles>

$5 a$

A white solid, (90 mg, 65\% yield). Eluent: petroleum ether/ethyl acetate $=2: 1 .{ }^{1} \mathbf{H}$ NMR $\left(400 \mathrm{MHz}, \mathrm{CDCl}_{3}\right) \delta 7.77-7.70(\mathrm{~m}, 2 \mathrm{H}), 7.56-$ $7.48(\mathrm{~m}, 3 \mathrm{H}), 7.45(\mathrm{~d}, \mathrm{~J}=12.6 \mathrm{~Hz}, 4 \mathrm{H}), 7.41(\mathrm{~s}, 6 \mathrm{H}), 7.35-7.28(\mathrm{~m}, 2 \mathrm{H})$, 
$7.22-7.15$ (m, 4H), $7.12-7.05$ (m, 4H), 6.95 (d, J = 8.1 Hz, 2H), $6.81-$ $6.42(\mathrm{~m}, 3 \mathrm{H}), 5.95(\mathrm{~d}, \mathrm{~J}=18.9 \mathrm{~Hz}, 1 \mathrm{H}), 2.29(\mathrm{~s}, 3 \mathrm{H}) .{ }^{13} \mathbf{C}$ NMR (101 MHz, $\left.\mathrm{CDCl}_{3}\right) \delta 149.4(\mathrm{~d}, \mathrm{~J}=5.5 \mathrm{~Hz}), 144.5(\mathrm{~d}, \mathrm{~J}=3.0 \mathrm{~Hz}), 144.0,143.3,142.4$, $141.8,141.1,140.2,138.6(\mathrm{~d}, \mathrm{~J}=10.0 \mathrm{~Hz}), 135.9,133.8,133.3,132.8(\mathrm{~d}$, $\mathrm{J}=11.1 \mathrm{~Hz}), 132.1(\mathrm{~d}, \mathrm{~J}=11.1 \mathrm{~Hz}), 131.8,131.7,131.7(\mathrm{~d}, \mathrm{~J}=10.1 \mathrm{~Hz})$, $131.6(\mathrm{~d}, \mathrm{~J}=11.1 \mathrm{~Hz}), 131.4(\mathrm{~d}, \mathrm{~J}=17.2 \mathrm{~Hz}), 130.7,128.8,128.8,128.4$ $(\mathrm{d}, \mathrm{J}=20.2 \mathrm{~Hz}), 128.3$ (d, J = 14.1 Hz), $128.1(\mathrm{~d}, \mathrm{~J}=12.1 \mathrm{~Hz}), 127.9$ (d, J $=18.2 \mathrm{~Hz}), 127.8,21.6 .{ }^{31} \mathbf{P}$ NMR $\left(162 \mathrm{MHz}, \mathrm{CDCl}_{3}\right) \delta 27.96(\mathrm{~s}) . \mathbf{H R M S}$ (ESI): $\left([\mathrm{M}+\mathrm{H}]^{+}\right)$Calcd for $\mathrm{C}_{43} \mathrm{H}_{35} \mathrm{ClO}_{3} \mathrm{PS}^{+}$: 697.1728, Found: 697.1719 .

\section{(4-bromo-3-(diphenylmethylene)-5-phenyl-5-tosylpenta-1,4-dien-2-}

\section{yl)diphenylphosphine oxide (5b)}

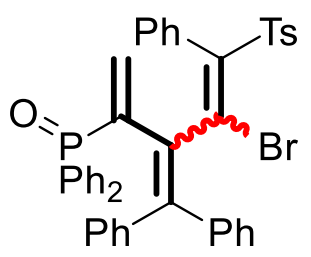

$5 b$

A white solid, (81 mg, 55\% yield). Eluent: petroleum ether/ethyl acetate $=2: 1 .{ }^{1} \mathbf{H}$ NMR $\left(400 \mathrm{MHz}, \mathrm{CDCl}_{3}\right) \delta 7.82-7.75(\mathrm{~m}, 2 \mathrm{H}), 7.59(\mathrm{~d}, \mathrm{~J}$ $=3.6 \mathrm{~Hz}, 2 \mathrm{H}), 7.55-7.43(\mathrm{~m}, 6 \mathrm{H}), 7.43-7.36(\mathrm{~m}, 6 \mathrm{H}), 7.35-7.30(\mathrm{~m}$, 2H), $7.22-7.12(\mathrm{~m}, 6 \mathrm{H}), 7.09(\mathrm{~d}, \mathrm{~J}=6.7 \mathrm{~Hz}, 2 \mathrm{H}), 6.96(\mathrm{~d}, \mathrm{~J}=8.0 \mathrm{~Hz}, 2 \mathrm{H})$, $6.74(\mathrm{~d}, \mathrm{~J}=9.0 \mathrm{~Hz}, 1 \mathrm{H}), 6.56(\mathrm{~d}, \mathrm{~J}=71.6 \mathrm{~Hz}, 1 \mathrm{H}), 5.95(\mathrm{~d}, \mathrm{~J}=18.9 \mathrm{~Hz}$, 1H), $2.30(\mathrm{~s}, 3 \mathrm{H}) .{ }^{13} \mathbf{C}$ NMR (101 MHz, $\left.\mathrm{CDCl}_{3}\right) \delta 148.8(\mathrm{~d}, \mathrm{~J}=5.4 \mathrm{~Hz})$, 144.0, 143.2 , 142.3, 141.9, 141.3, 138.7 (d, J = 10.9 Hz), 138.2 (d, J = 2.6 $\mathrm{Hz}), 136.0,135.6,134.0,133.0(\mathrm{~d}, \mathrm{~J}=5.8 \mathrm{~Hz}), 132.2(\mathrm{~d}, \mathrm{~J}=9.5 \mathrm{~Hz}), 131.7$, $131.7(\mathrm{~d}, \mathrm{~J}=9.1 \mathrm{~Hz}), 131.5(\mathrm{~d}, \mathrm{~J}=3.0 \mathrm{~Hz}), 131.3(\mathrm{~d}, \mathrm{~J}=9.5 \mathrm{~Hz}), 130.8$, 128.9, 128.8, 128.5, $128.2(\mathrm{~d}, \mathrm{~J}=7.1 \mathrm{~Hz}), 128.1(\mathrm{~d}, \mathrm{~J}=11.1 \mathrm{~Hz}), 127.9$, 127.7, 127.7, 21.6. ${ }^{31}$ P NMR (162 MHz, $\left.\mathrm{CDCl}_{3}\right) \delta 27.66$ (s). HRMS (ESI): $\left([\mathrm{M}+\mathrm{H}]^{+}\right)$Calcd for $\mathrm{C}_{43} \mathrm{H}_{35} \mathrm{BrO}_{3} \mathrm{PS}^{+}:$741.1222, Found: 741.1216 . 
12. ${ }^{1} \mathrm{H}-\mathrm{NMR},{ }^{13} \mathrm{C}-\mathrm{NMR},{ }^{31} \mathrm{P}-\mathrm{NMR},{ }^{19} \mathrm{~F}-\mathrm{NMR}$, and HRMS spectra for Substrates and Products

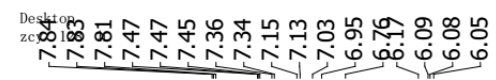

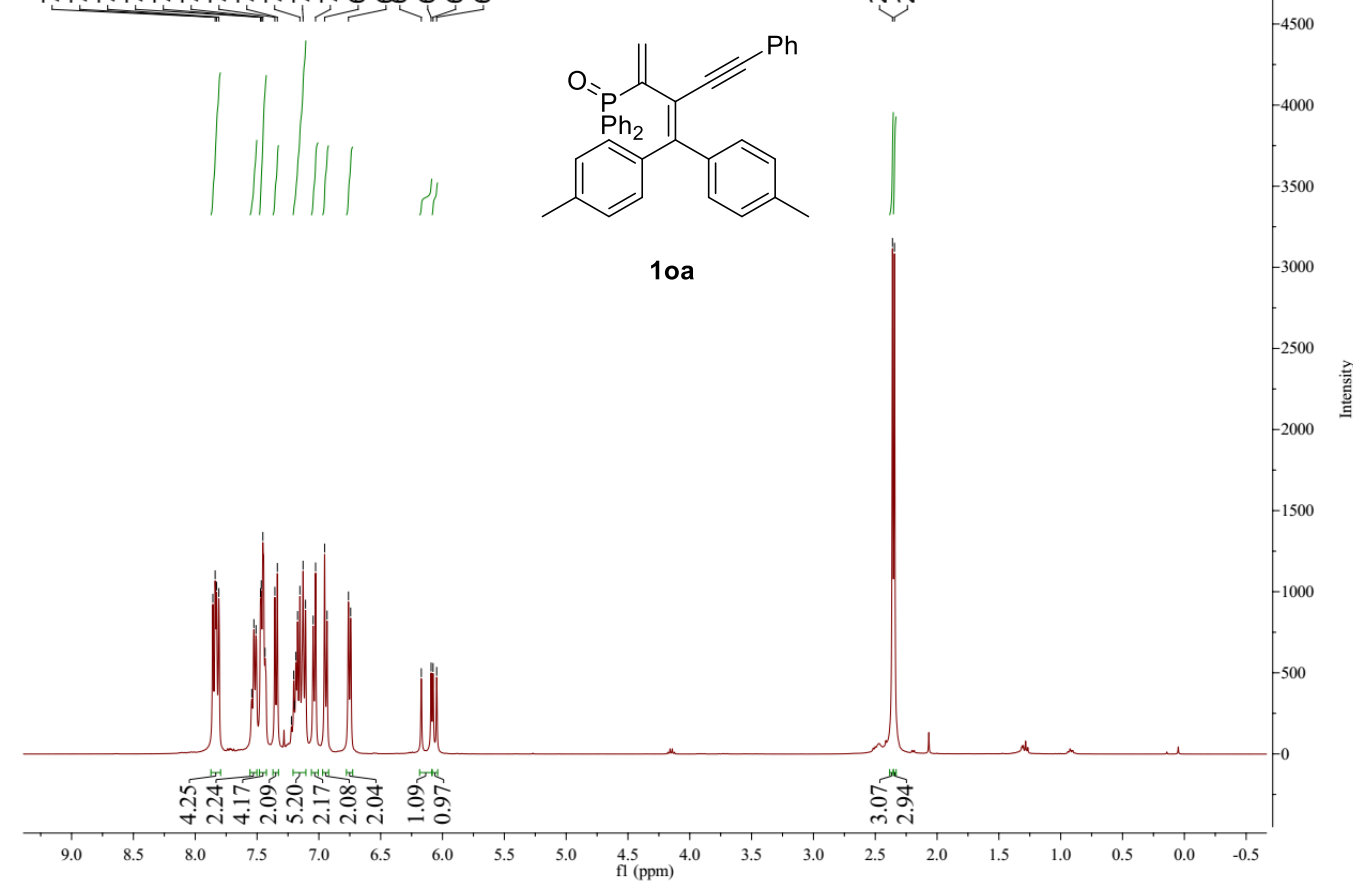

${ }^{1} \mathrm{H}$ NMR (400 MHz, $\left.\mathrm{CDCl}_{3}\right)$ of $\mathbf{1 0 a}$

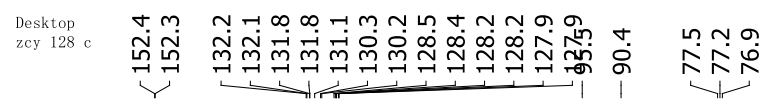

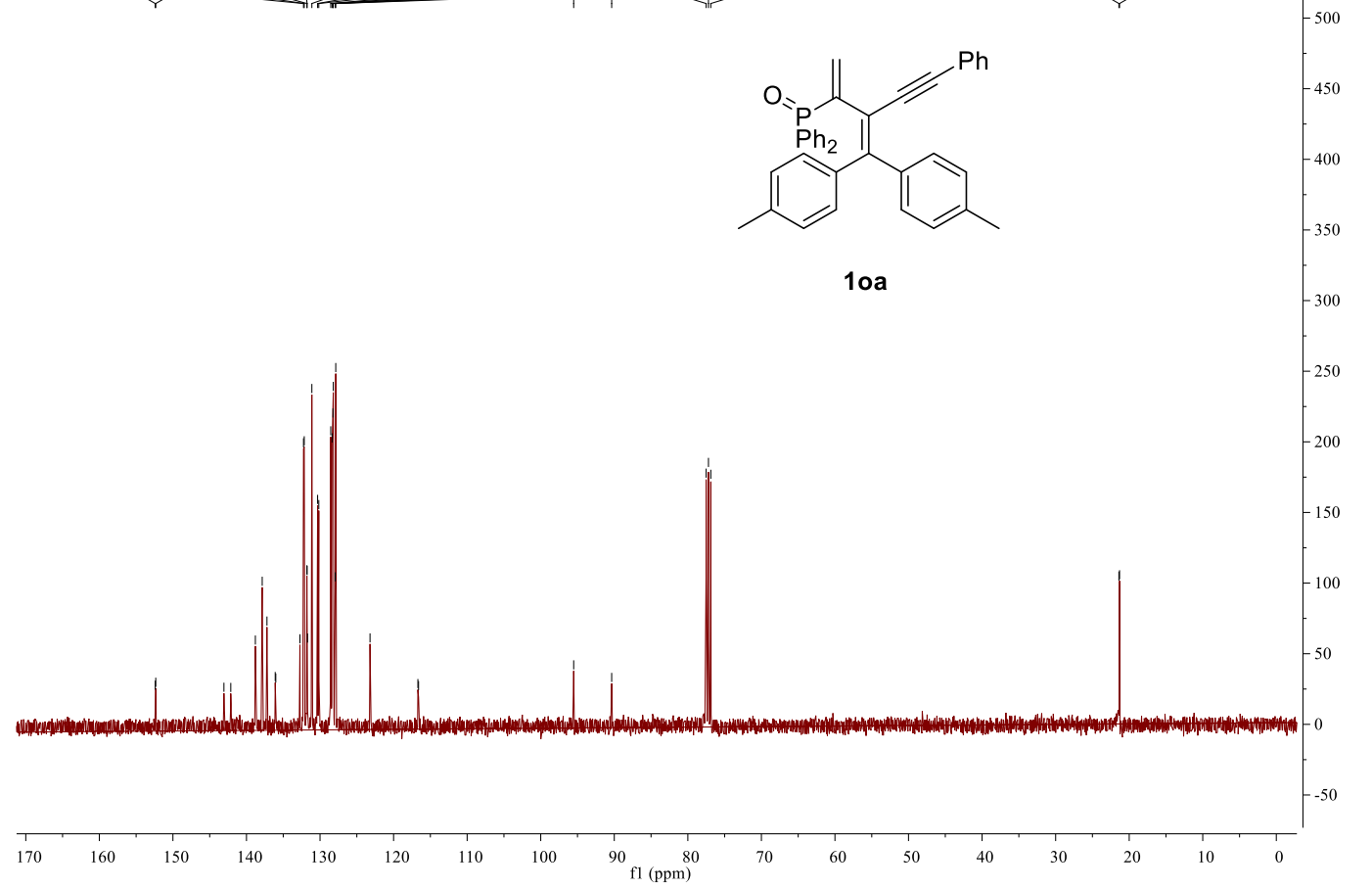

${ }^{13} \mathrm{C}$ NMR $\left(101 \mathrm{MHz}, \mathrm{CDCl}_{3}\right)$ of $\mathbf{1 0 a}$ 


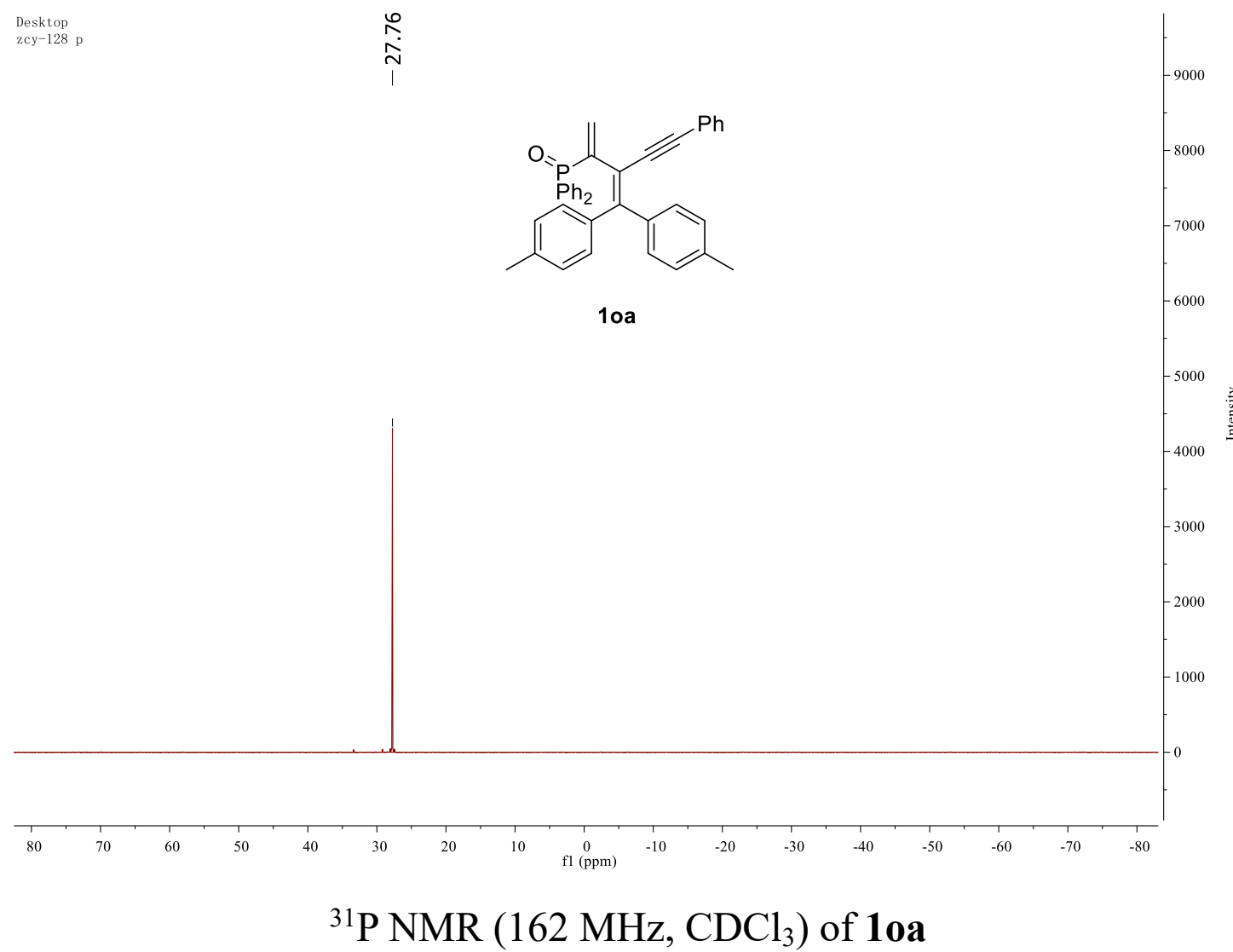

15 \#13 RT: 0.19 AV: 1 NL: 8.70E6

T: FTMS $\{1,1\}+p$ ESI Full ms [100.00-1000.00]

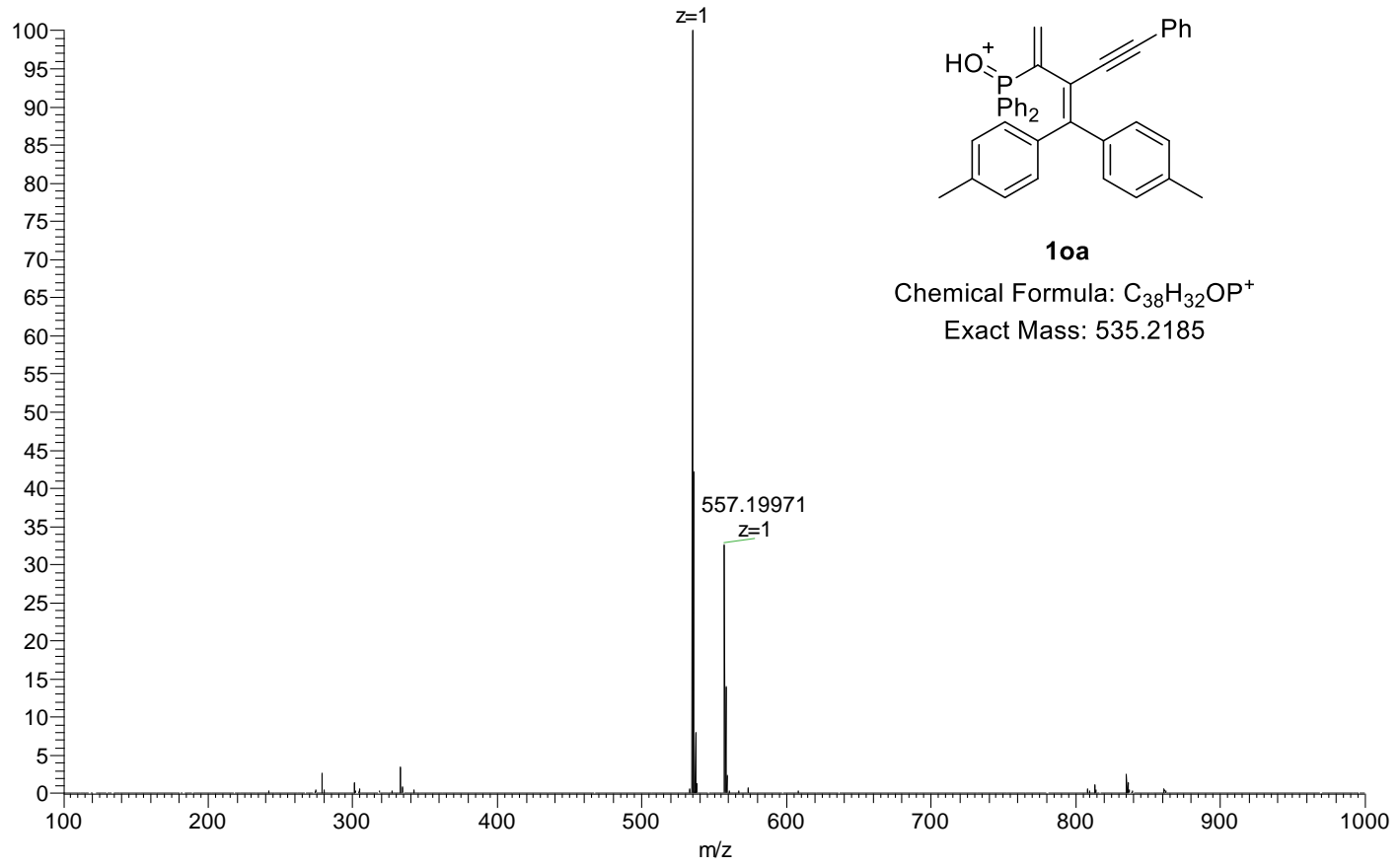




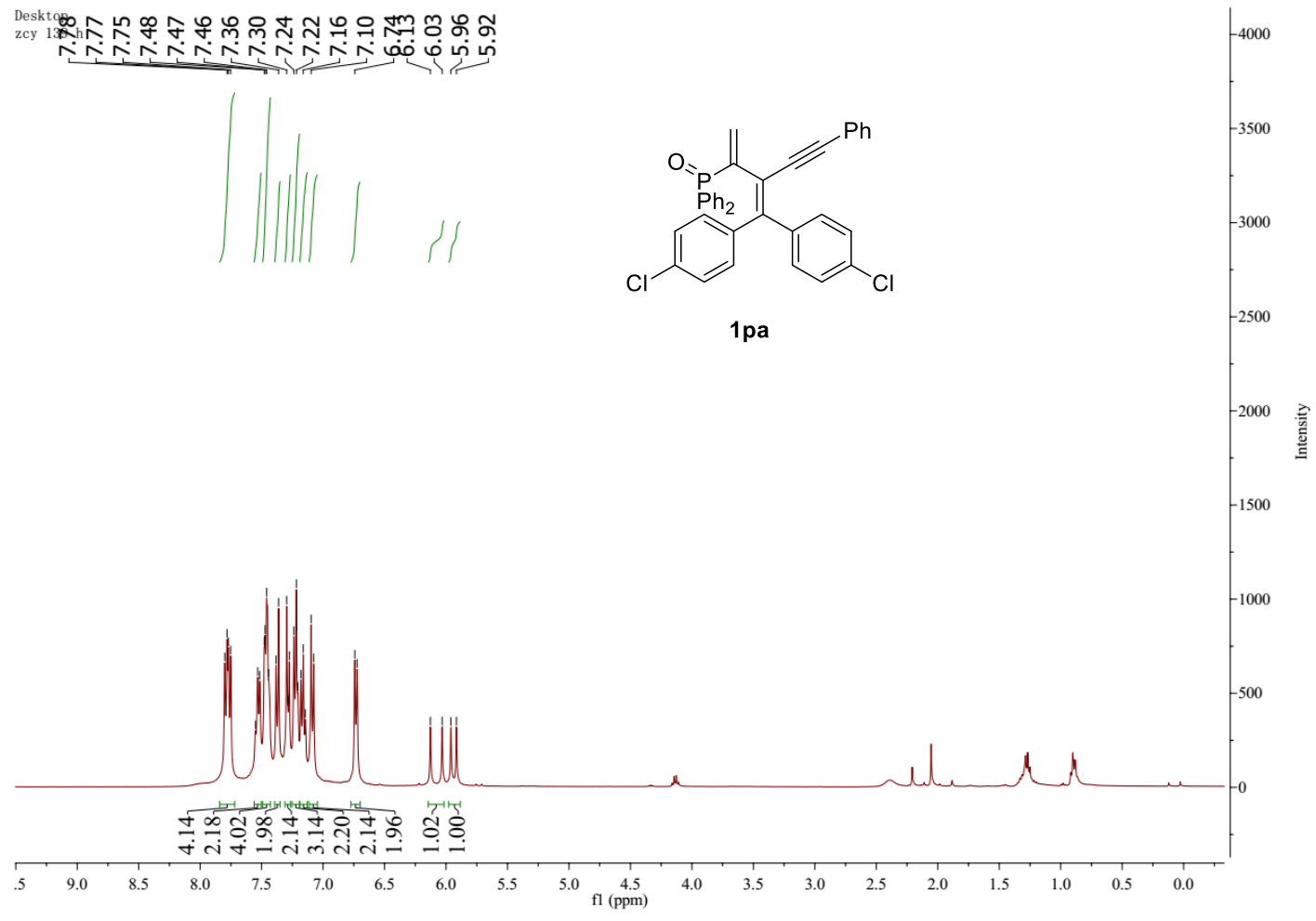

\section{${ }^{1} \mathrm{H}$ NMR $\left(400 \mathrm{MHz}, \mathrm{CDCl}_{3}\right)$ of $\mathbf{1}$ pa}

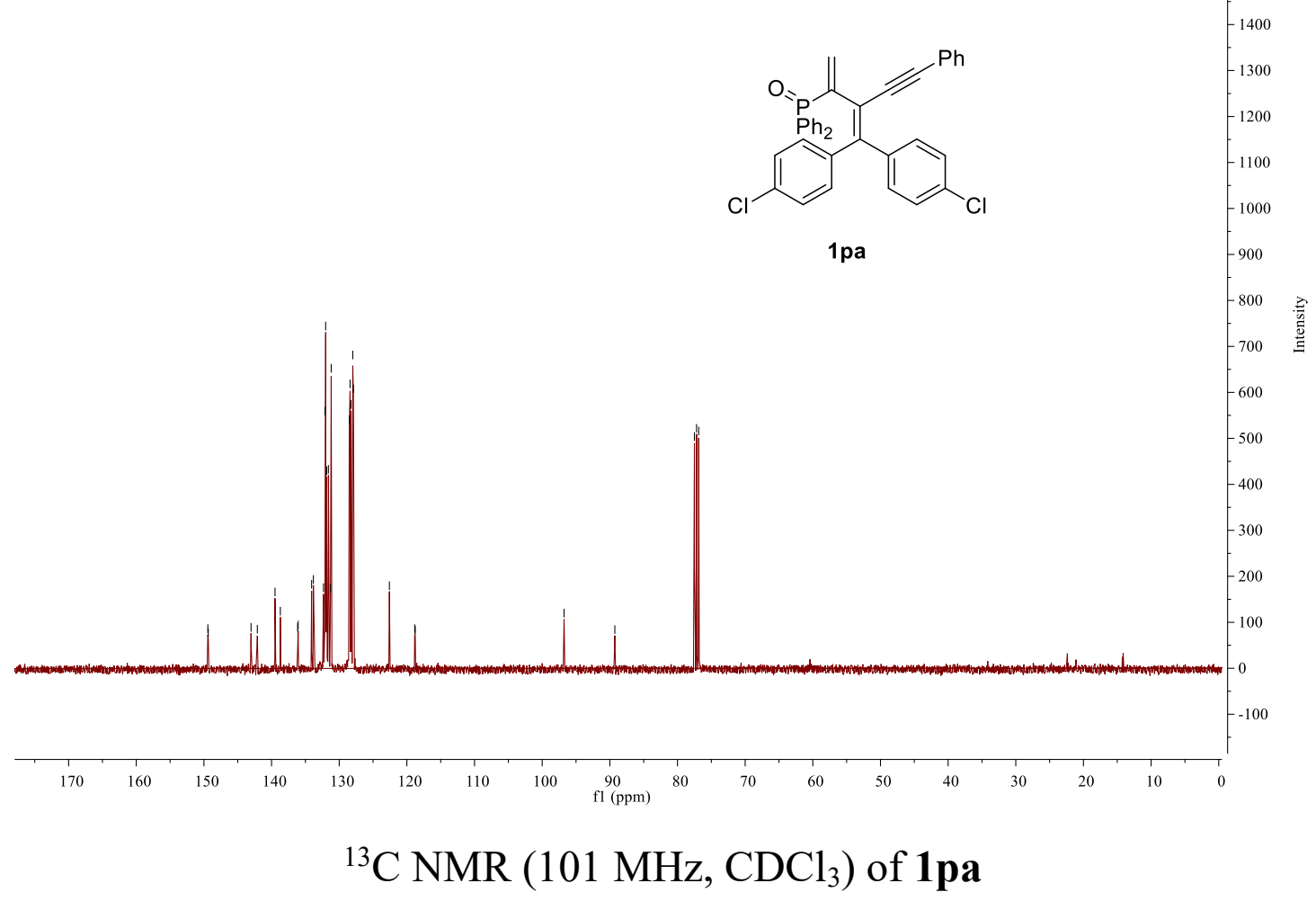




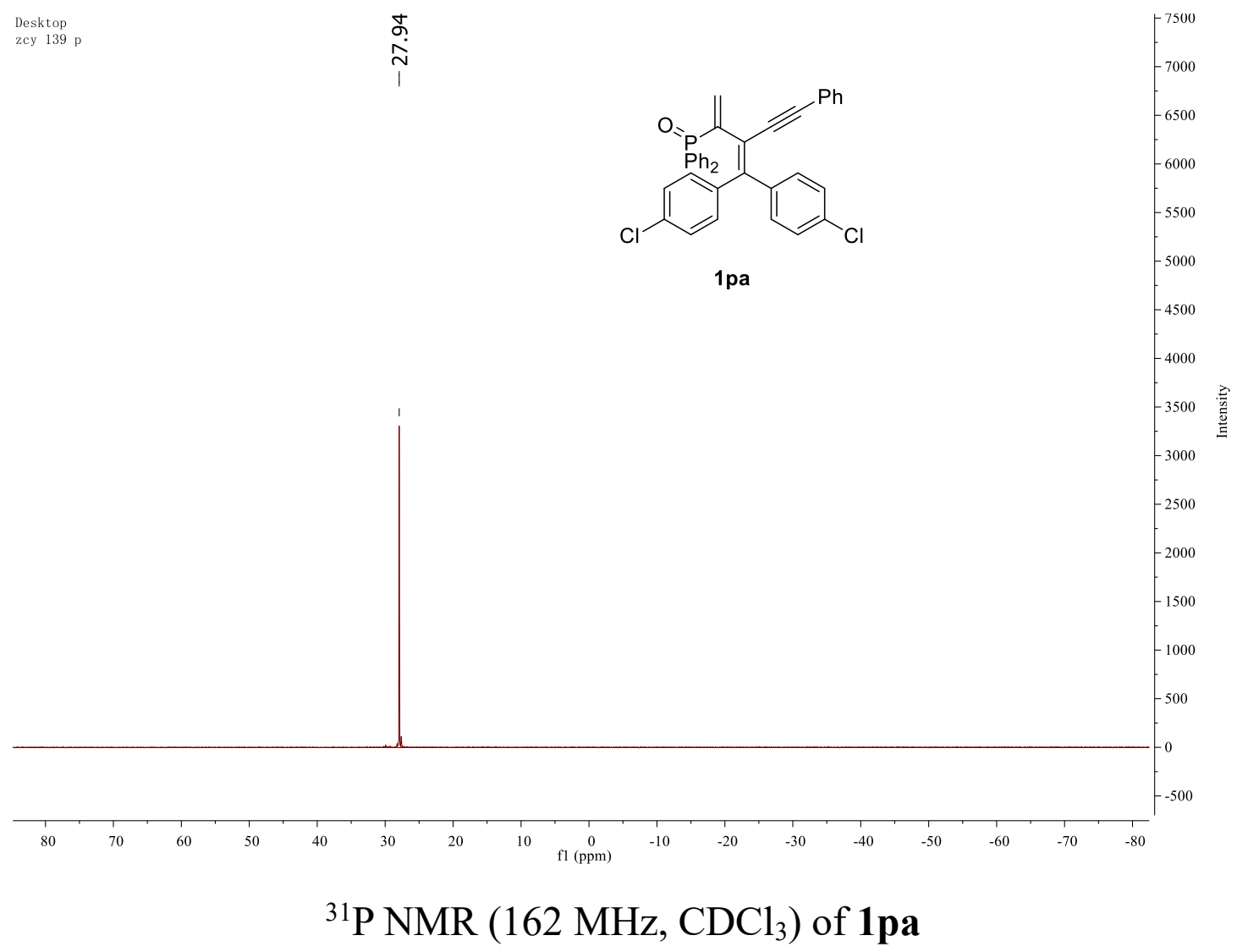

21 \#21 RT: 0.32 AV: 1 NL: $1.84 E 5$

T: FTMS $\{1,1\}+$ PESI Full ms [100.00-1000.00]

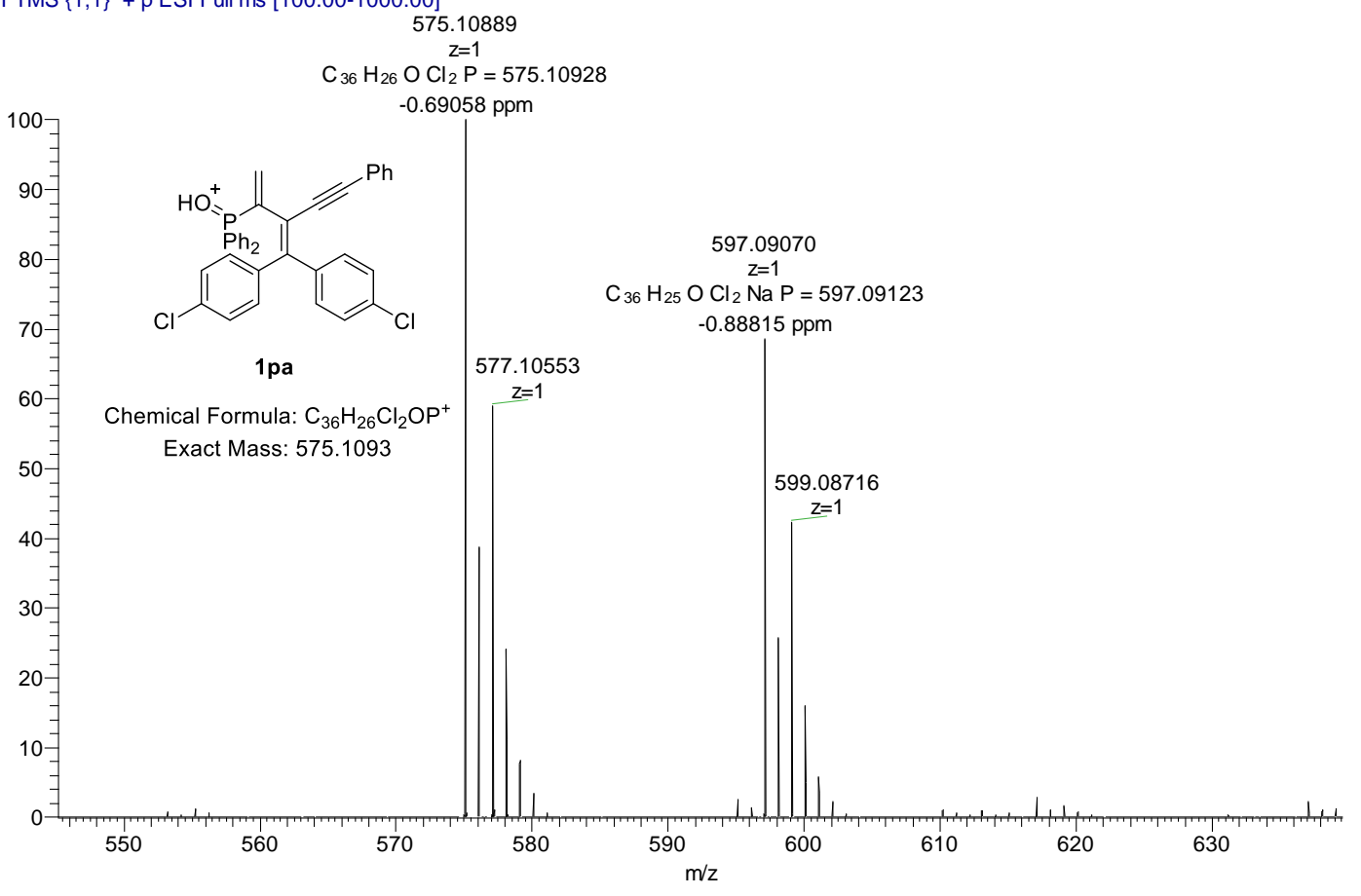




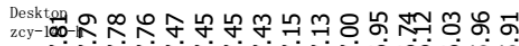

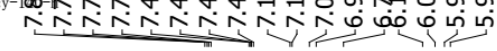
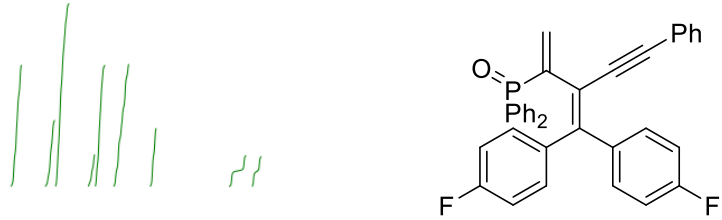

$1 q a$

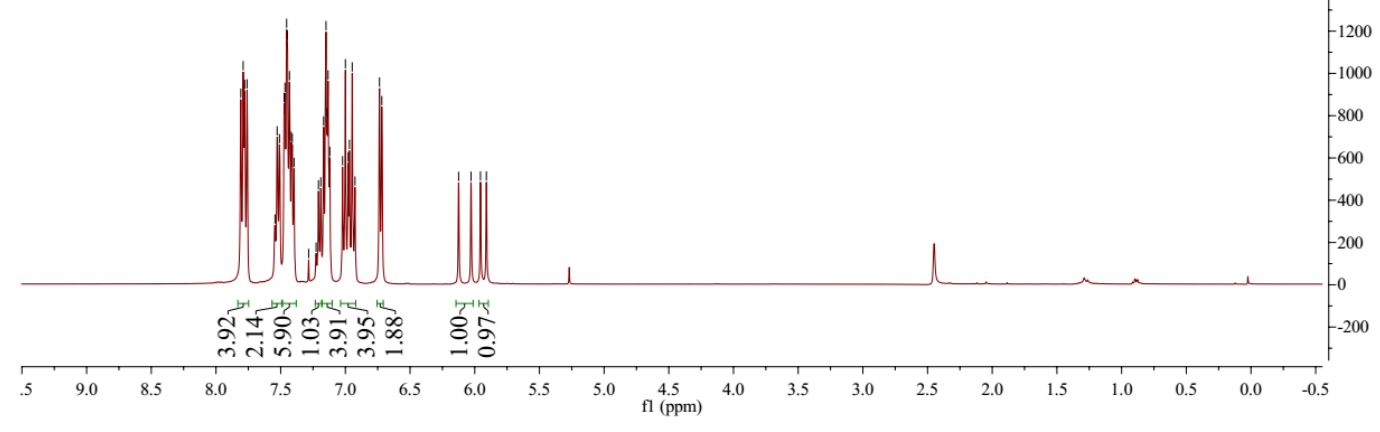

${ }^{1} \mathrm{H}$ NMR (400 MHz, $\left.\mathrm{CDCl}_{3}\right)$ of 1 qa

Deskopo

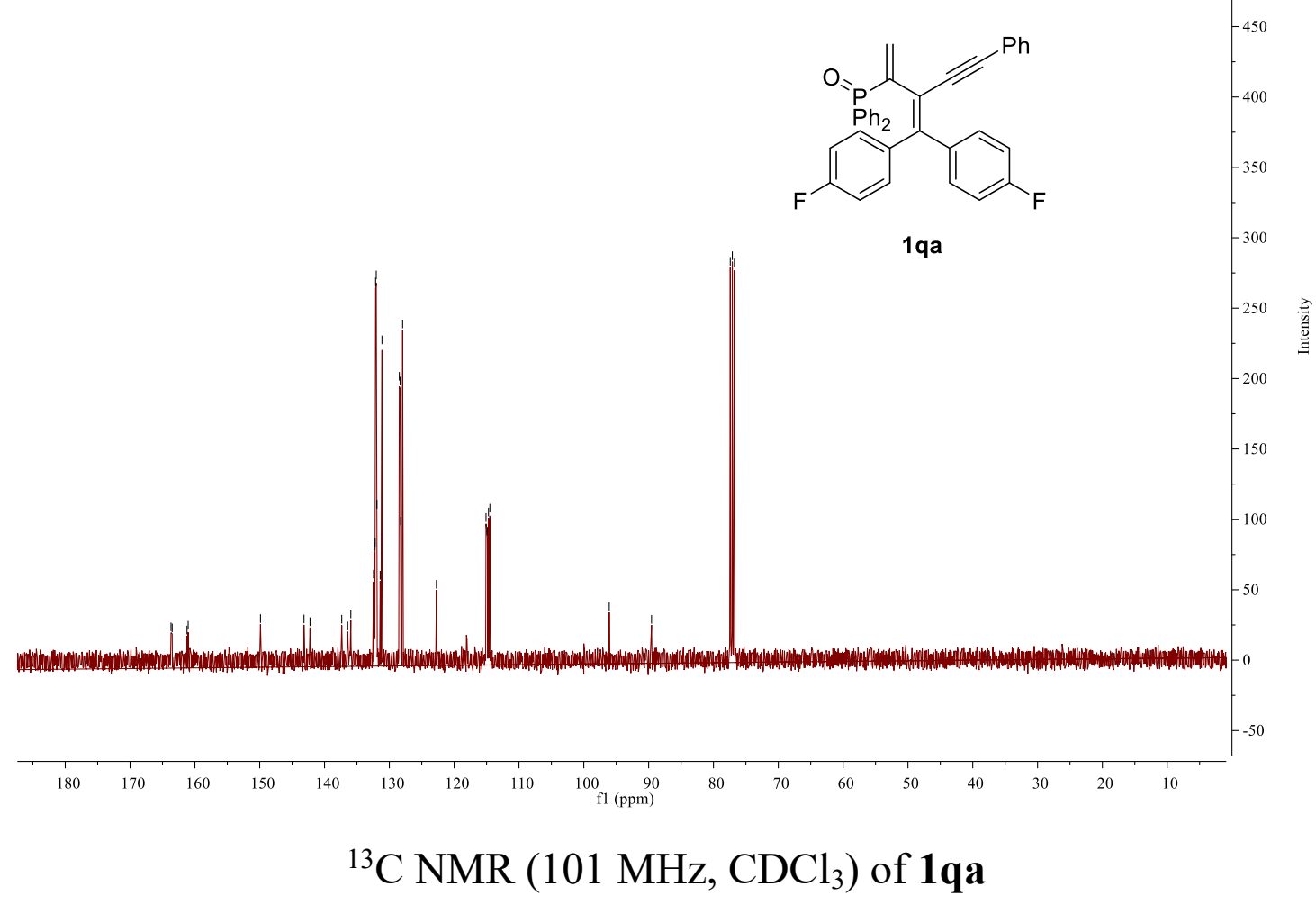




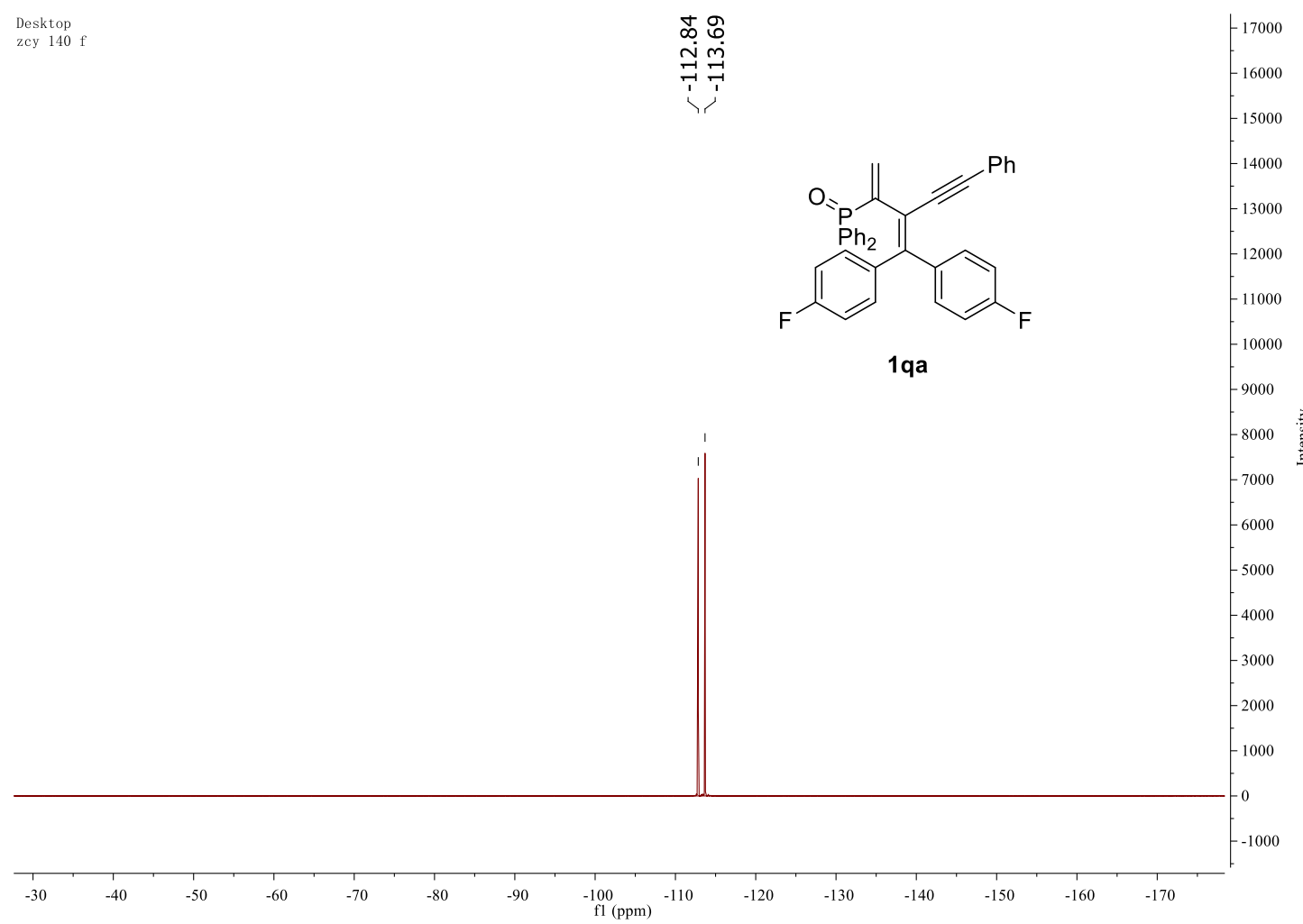

\section{${ }^{19} \mathrm{~F}$ NMR $\left(376 \mathrm{MHz}, \mathrm{CDCl}_{3}\right)$ of $\mathbf{1 q a}$}

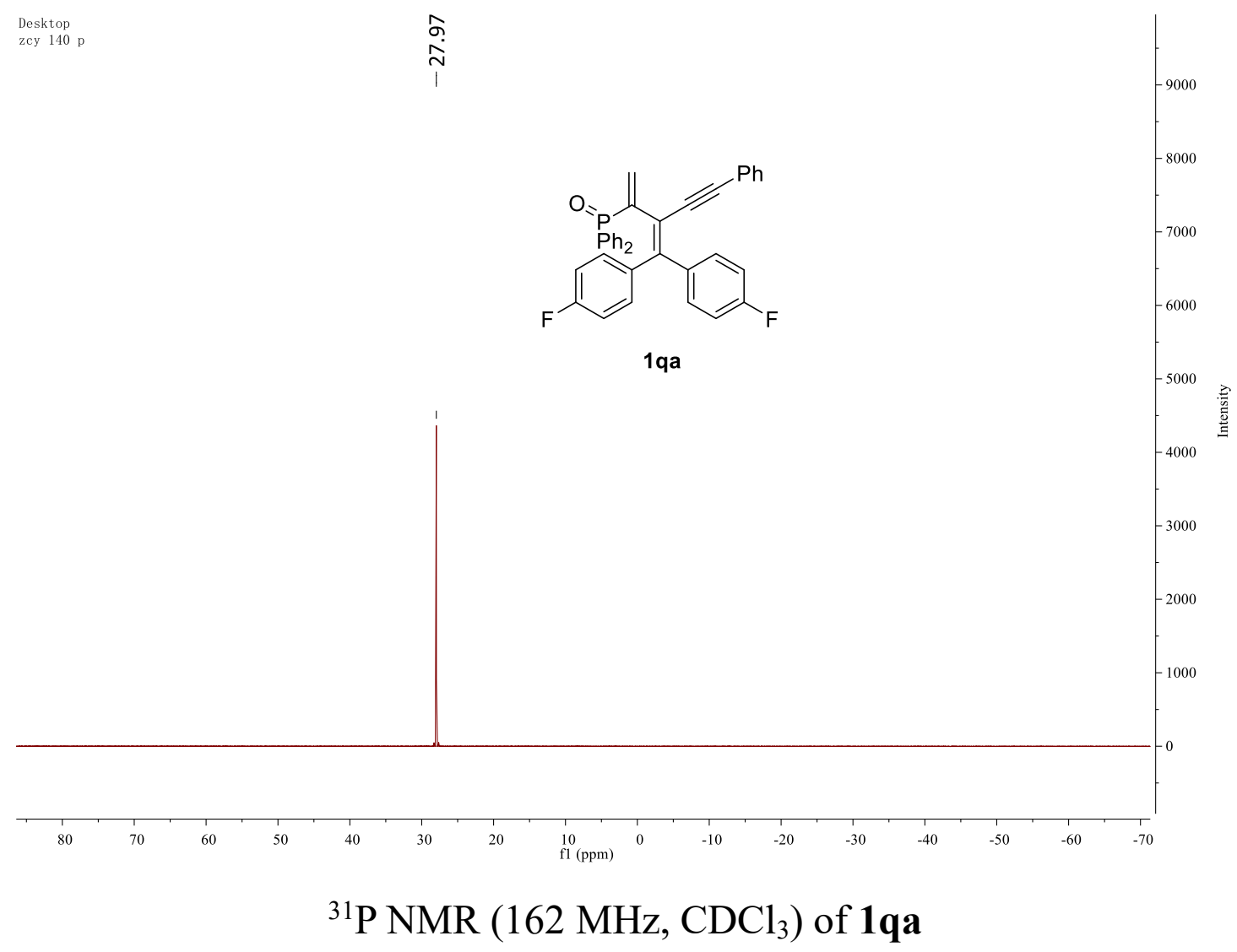


22 \#23 RT: 0.34 AV: 1 NL: $4.78 E 5$

T: FTMS $\{1,1\}+p$ ESI Full ms [100.00-1000.00] 543.16803

$\mathrm{C}_{36} \mathrm{H}_{26} \mathrm{O} \mathrm{F}_{2} \mathrm{P}=543.16838$

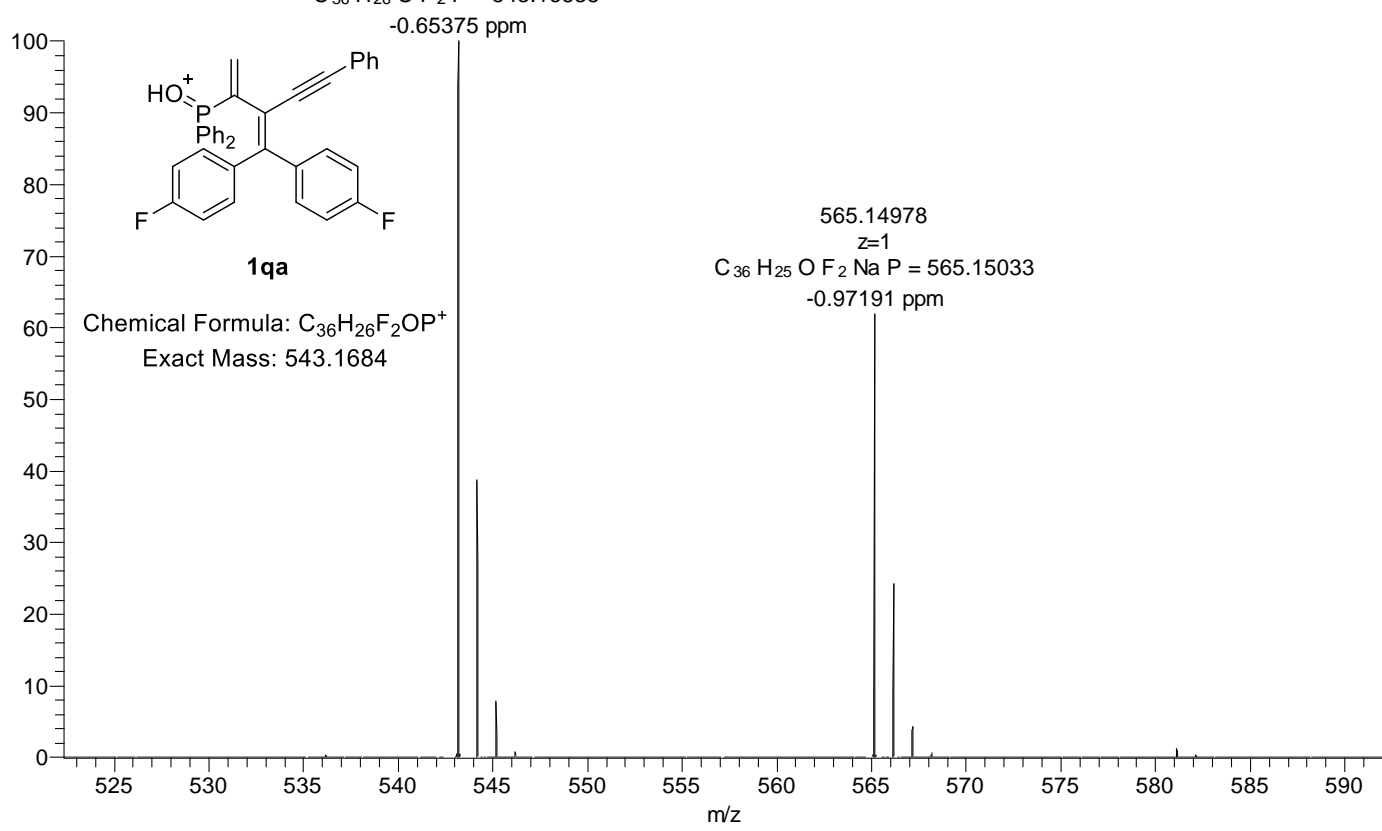

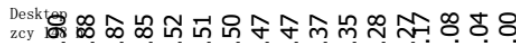

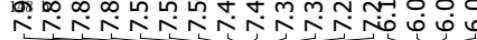
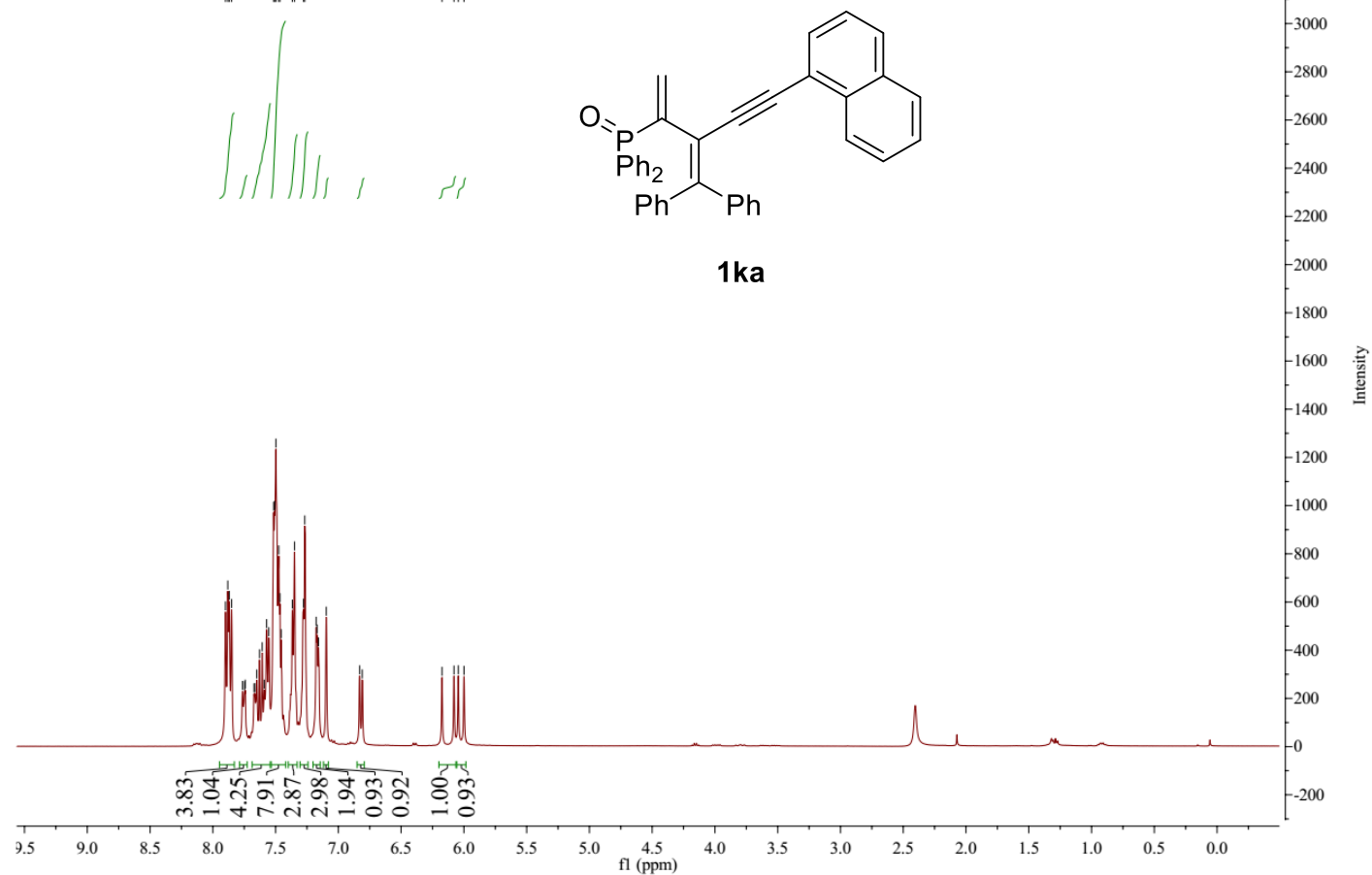

$-3200$

${ }^{1} \mathrm{H}$ NMR $\left(400 \mathrm{MHz}, \mathrm{CDCl}_{3}\right)$ of $\mathbf{1} \mathbf{k a}$ 


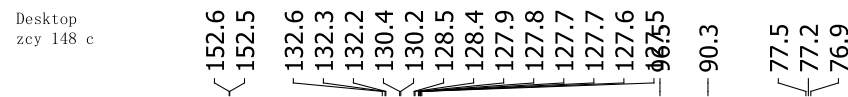
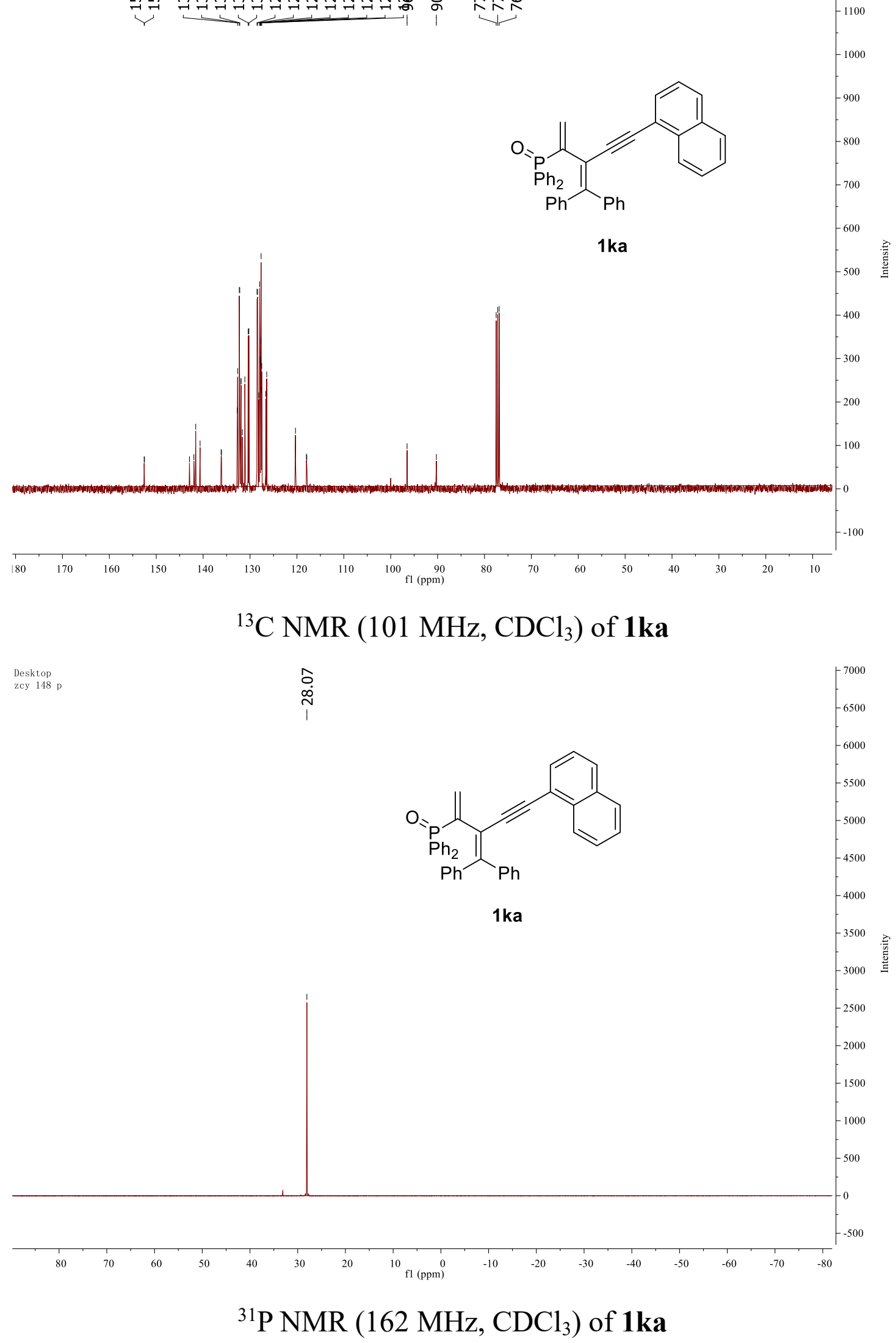
20190708-12 \#9 RT: 0.13 AV: 1 NL: 1.60E7

T: FTMS $\{1,1\}+p$ ESI Full ms [100.00-1000.00]
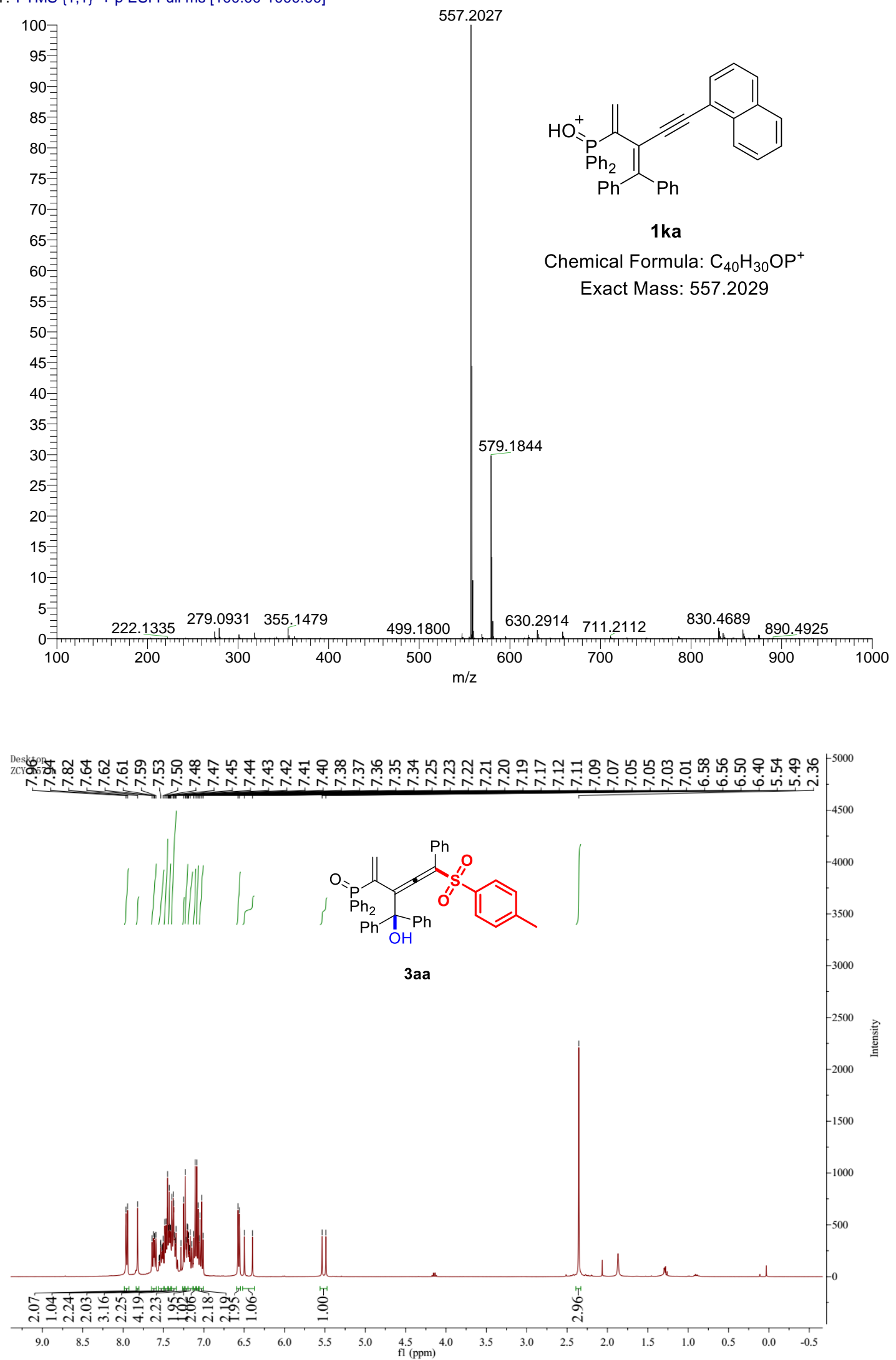

${ }^{1} \mathrm{H}$ NMR $\left(400 \mathrm{MHz}, \mathrm{CDCl}_{3}\right)$ of $\mathbf{3 a a}$ 


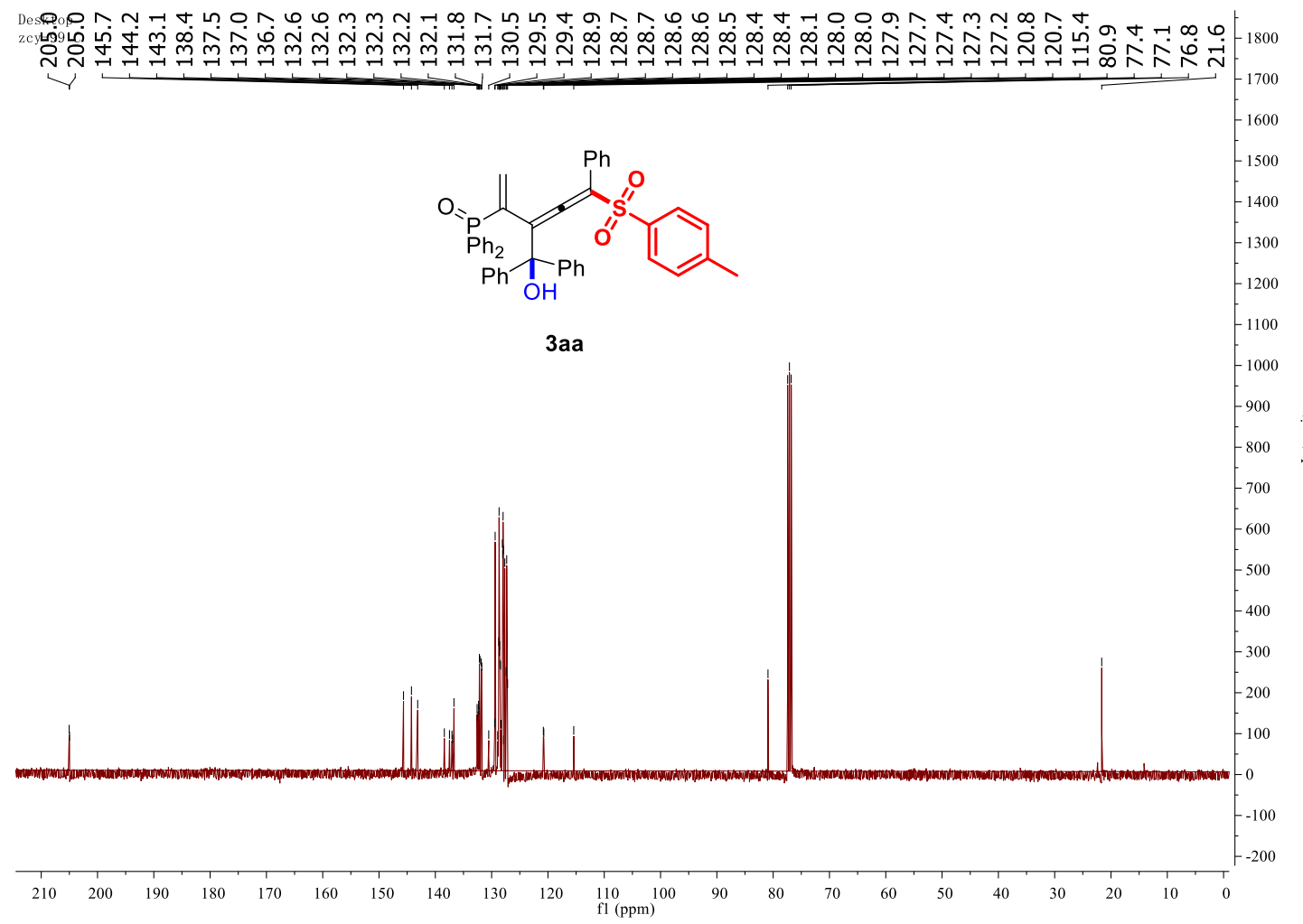

\section{${ }^{13} \mathrm{C}$ NMR $\left(101 \mathrm{MHz}, \mathrm{CDCl}_{3}\right)$ of $\mathbf{3 a a}$}

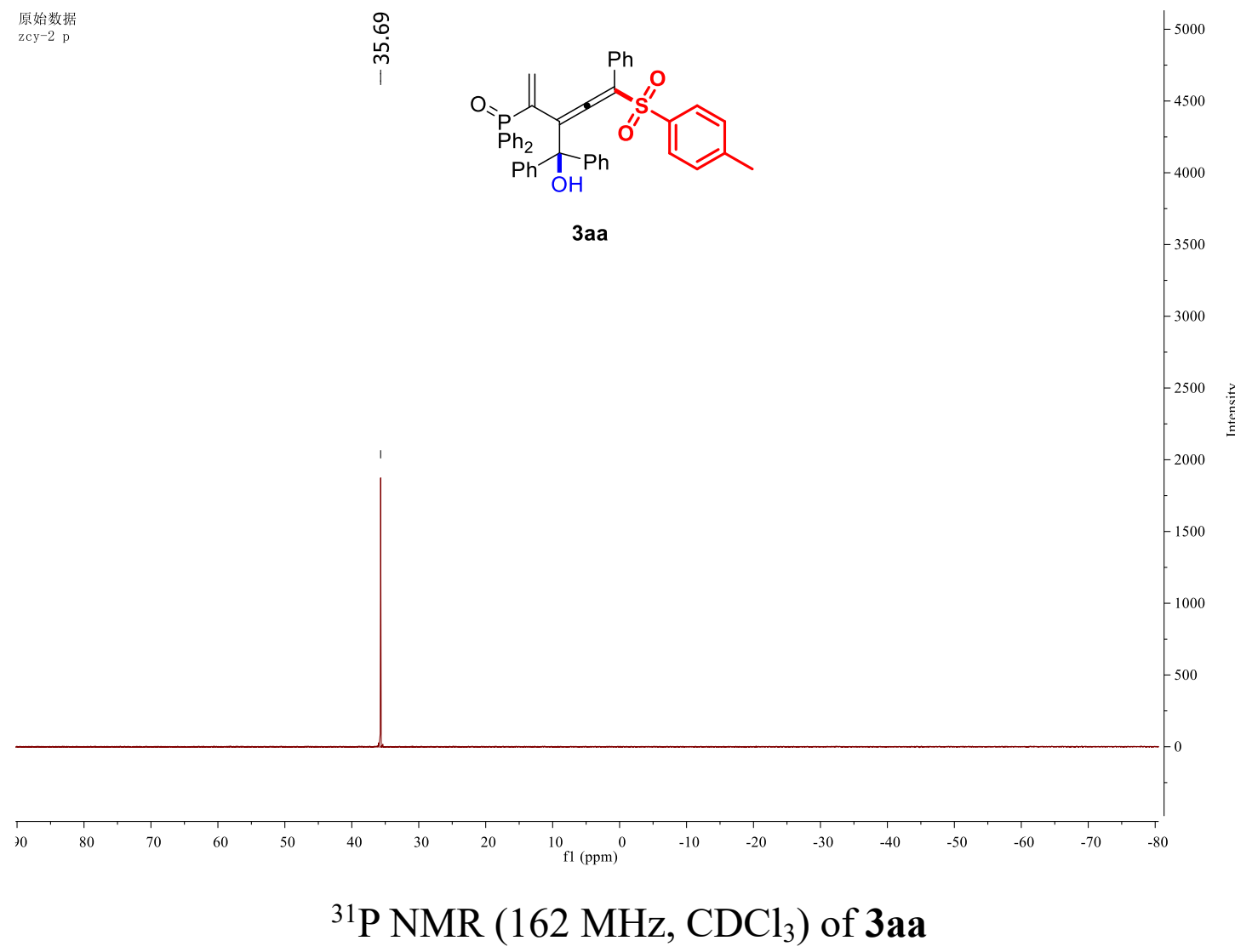



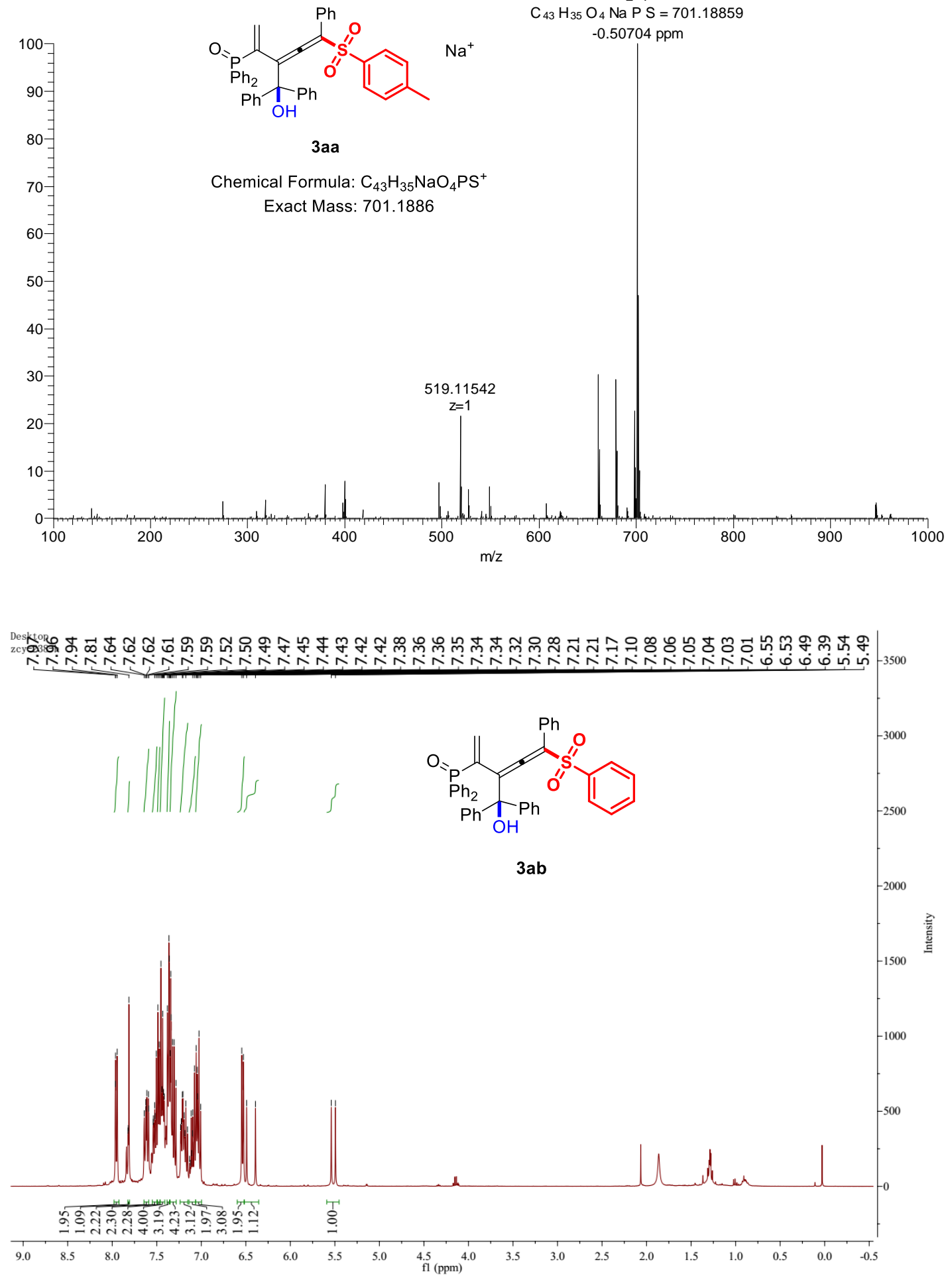

${ }^{1} \mathrm{H}$ NMR $\left(400 \mathrm{MHz}, \mathrm{CDCl}_{3}\right)$ of $\mathbf{3 a b}$ 


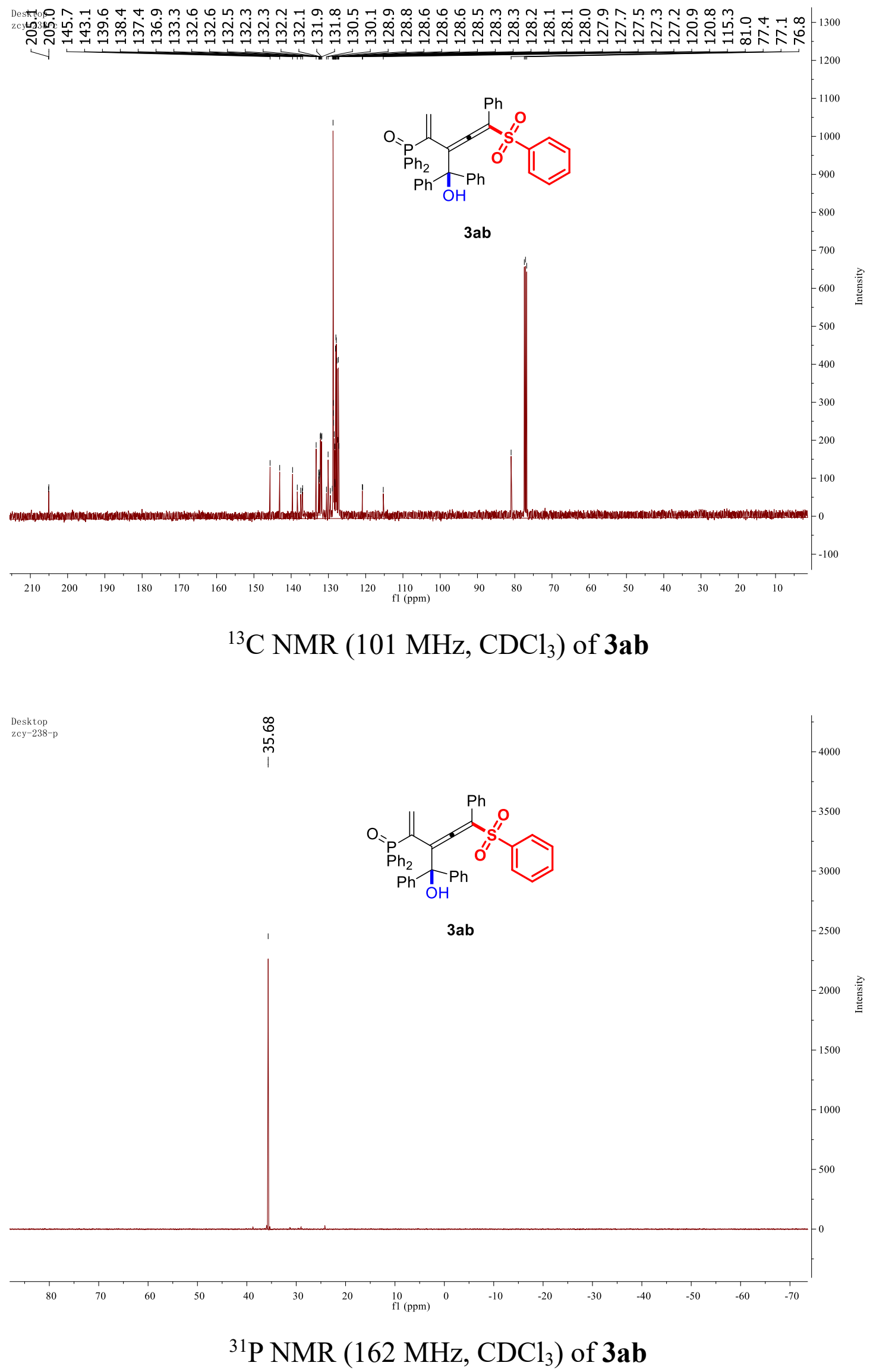


20200720-2 \#17 RT: 0.27 AV: 1 NL: 2.52E6

T: FTMS $\{1,1\}+p$ ESI Full ms [100.00-1000.00]
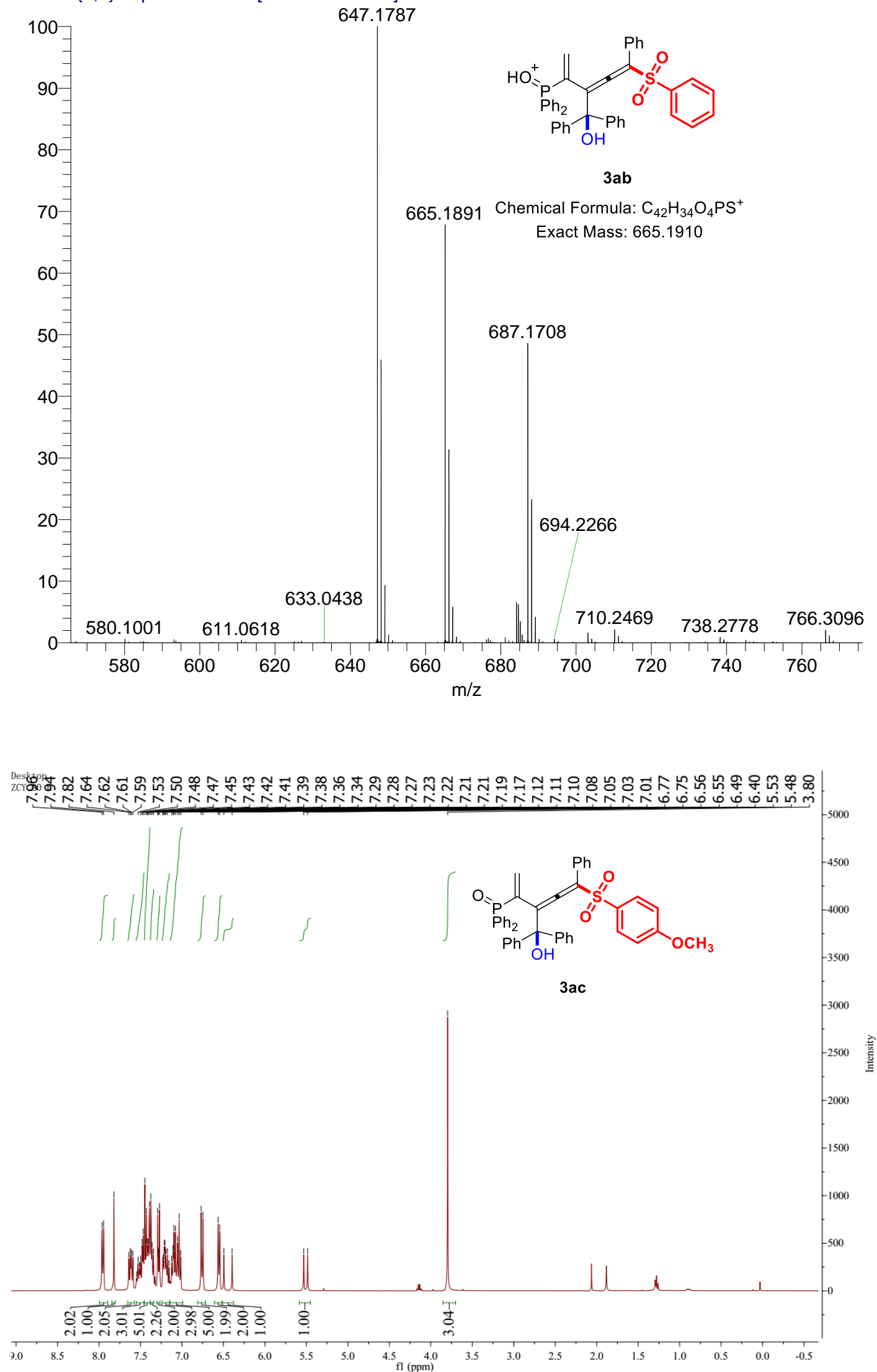

${ }^{1} \mathrm{H}$ NMR $\left(400 \mathrm{MHz}, \mathrm{CDCl}_{3}\right)$ of $\mathbf{3 a c}$ 


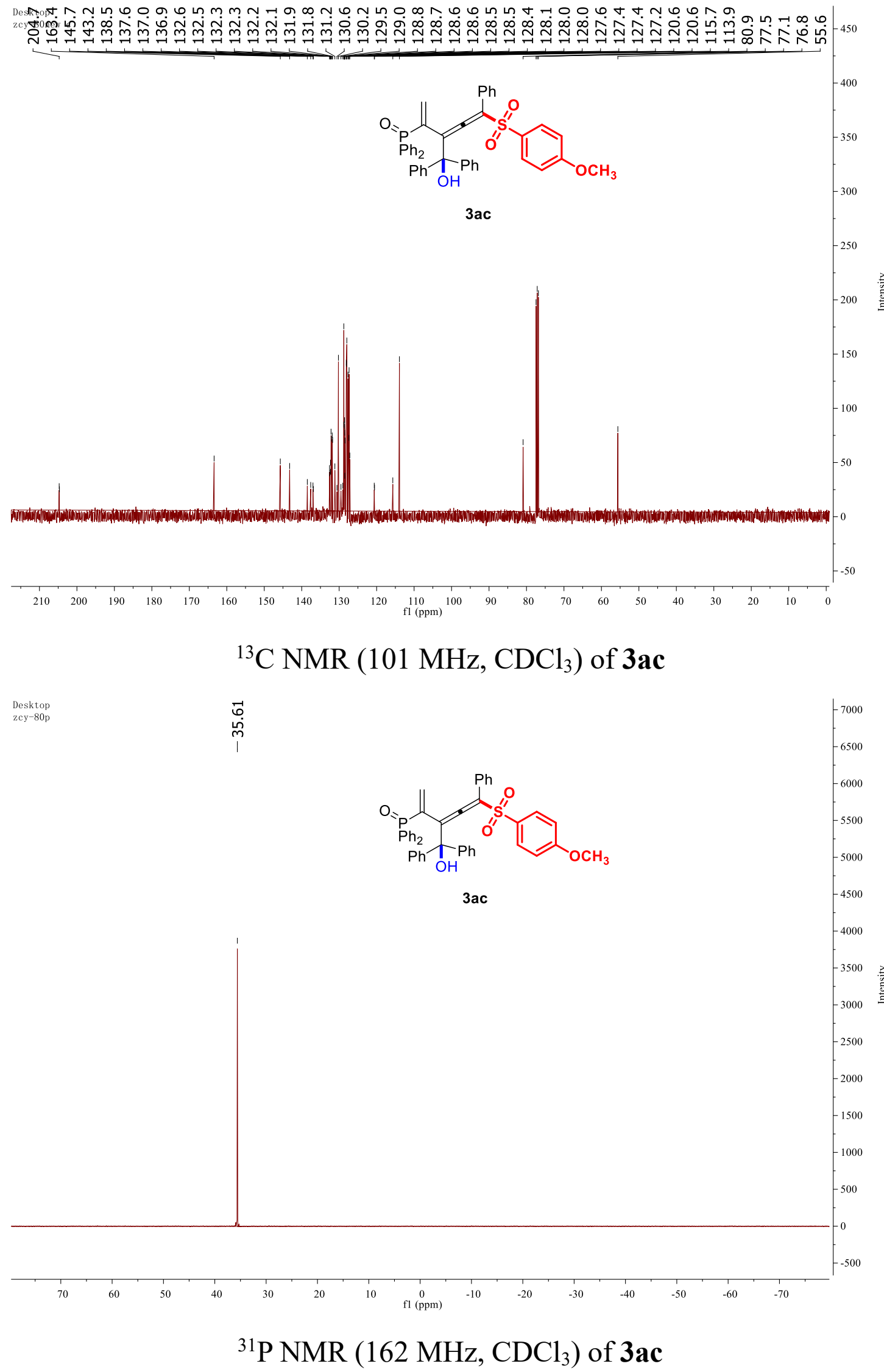


20190506-19 \#19 RT: 0.29 AV: 1 SB: 21 0.01-0.07, 0.78-1.04 NL: 1.97E5 T: FTMS $\{1,1\}+p$ ESI Full ms [100.00-1000.00]

$$
677.18998
$$

$\mathrm{C}_{43} \mathrm{H}_{34} \mathrm{O}_{4} \mathrm{P} \mathrm{S}=677.19099$
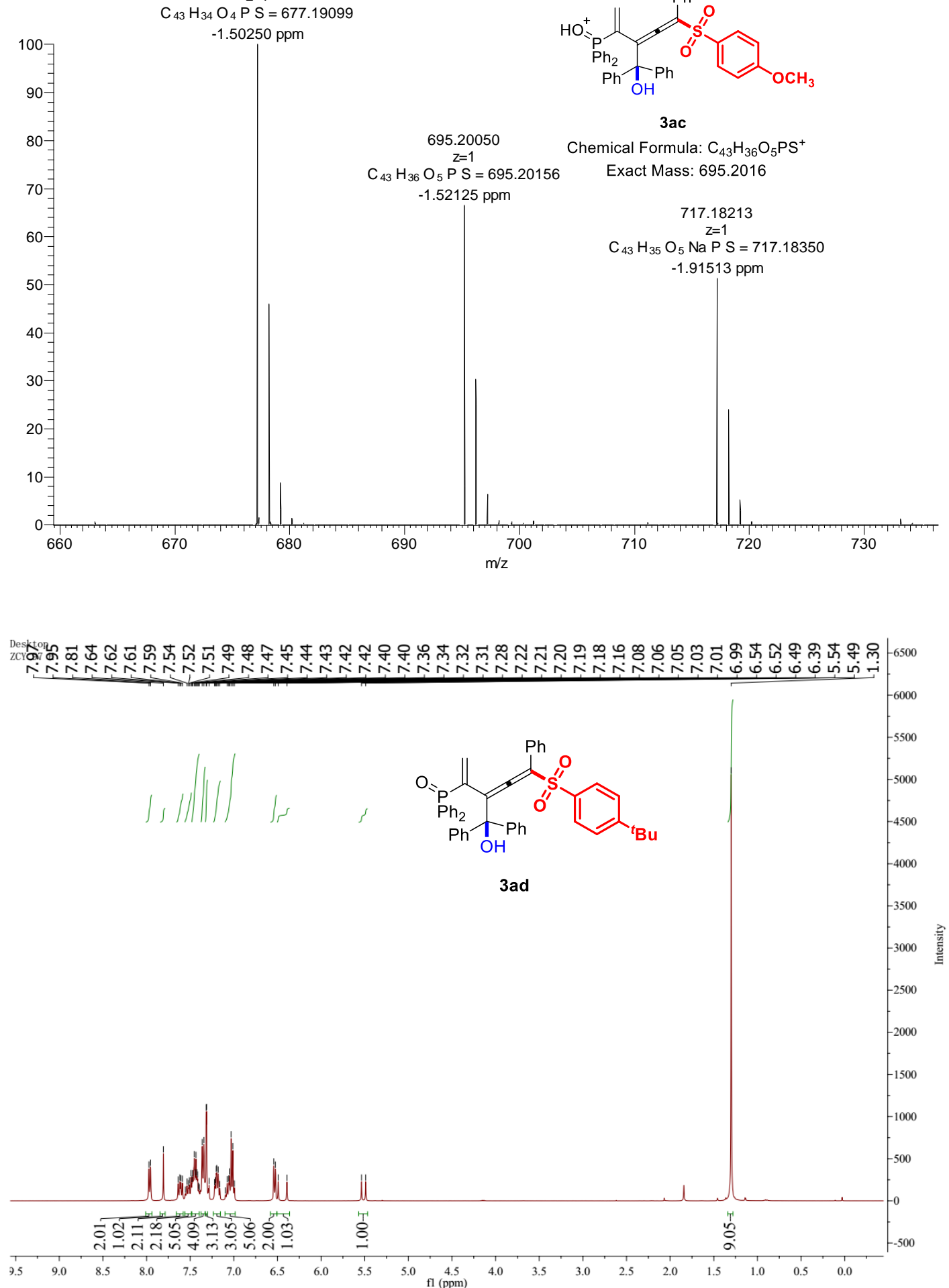

${ }^{1} \mathrm{H}$ NMR $\left(400 \mathrm{MHz}, \mathrm{CDCl}_{3}\right)$ of $\mathbf{3 a d}$ 


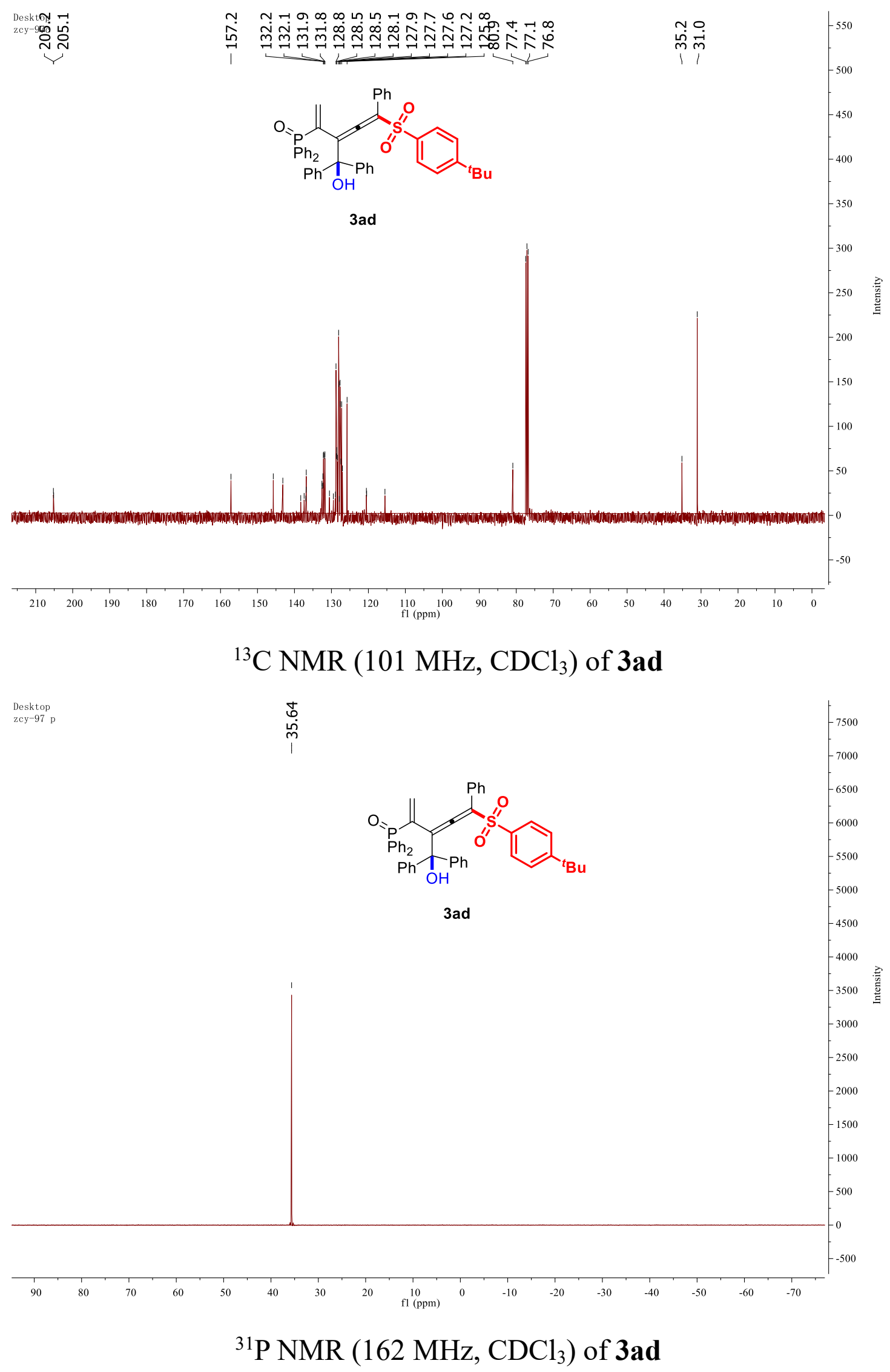


20190524-7 \#9 RT: 0.14 AV: 1 SB: 36 0.01-0.04, 0.48-1.03 NL: 8.28E6 T: FTMS $\{1,1\}+p$ ESI Full ms [100.00-1000.00]

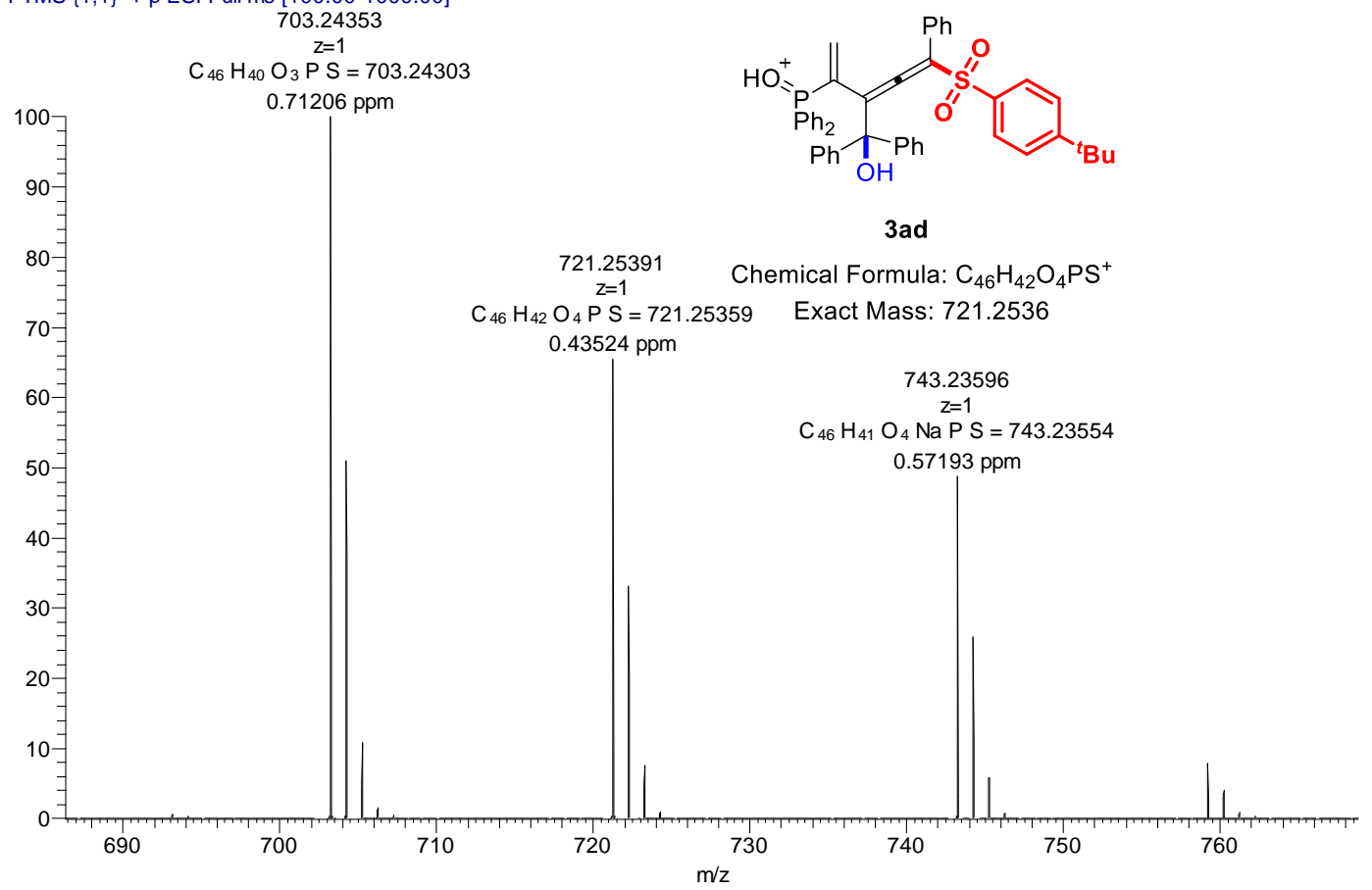

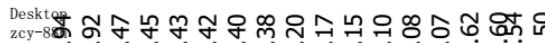

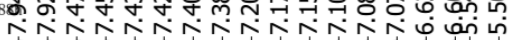
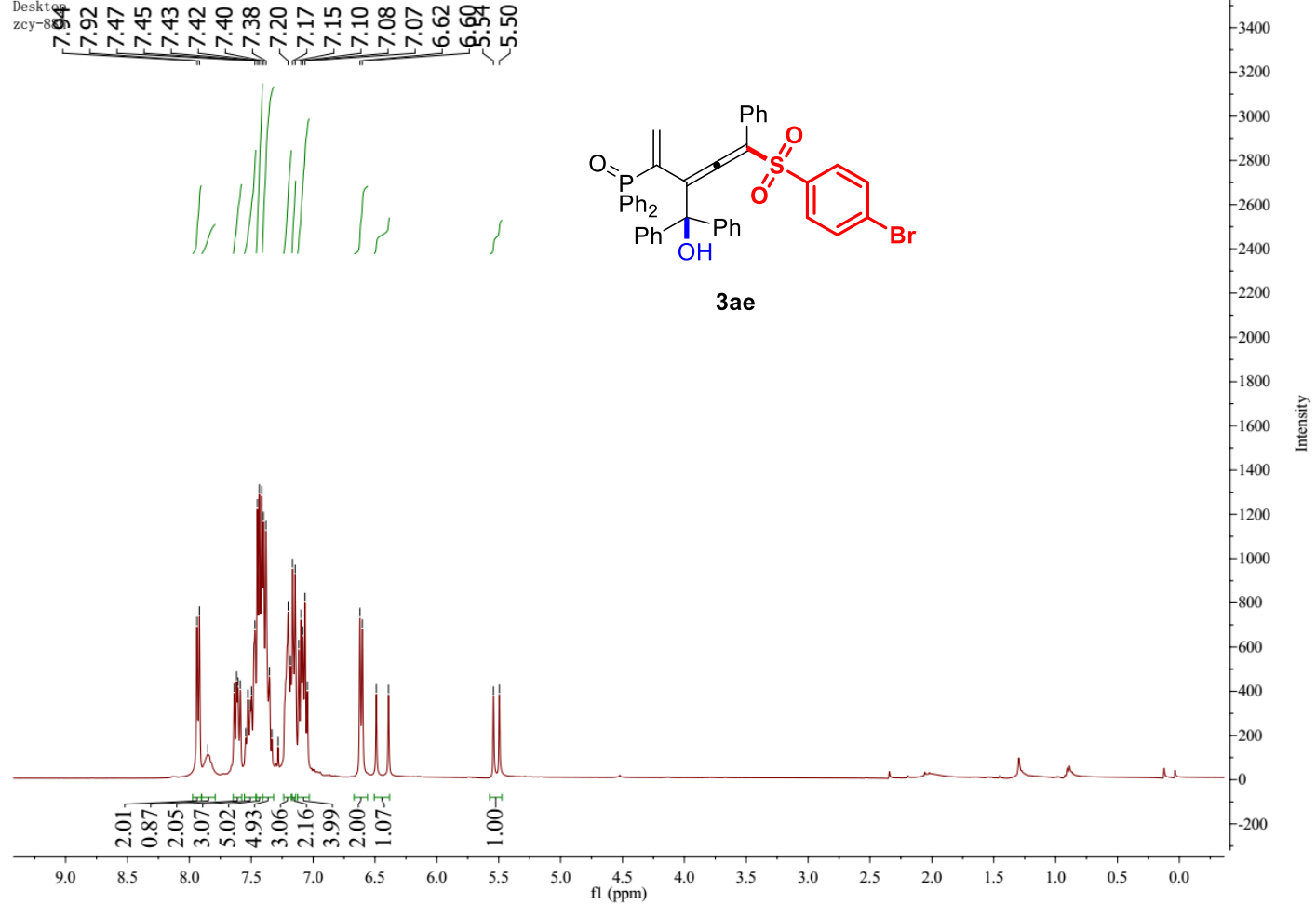

3400

${ }^{1} \mathrm{H}$ NMR $\left(400 \mathrm{MHz}, \mathrm{CDCl}_{3}\right)$ of $\mathbf{3 a e}$ 


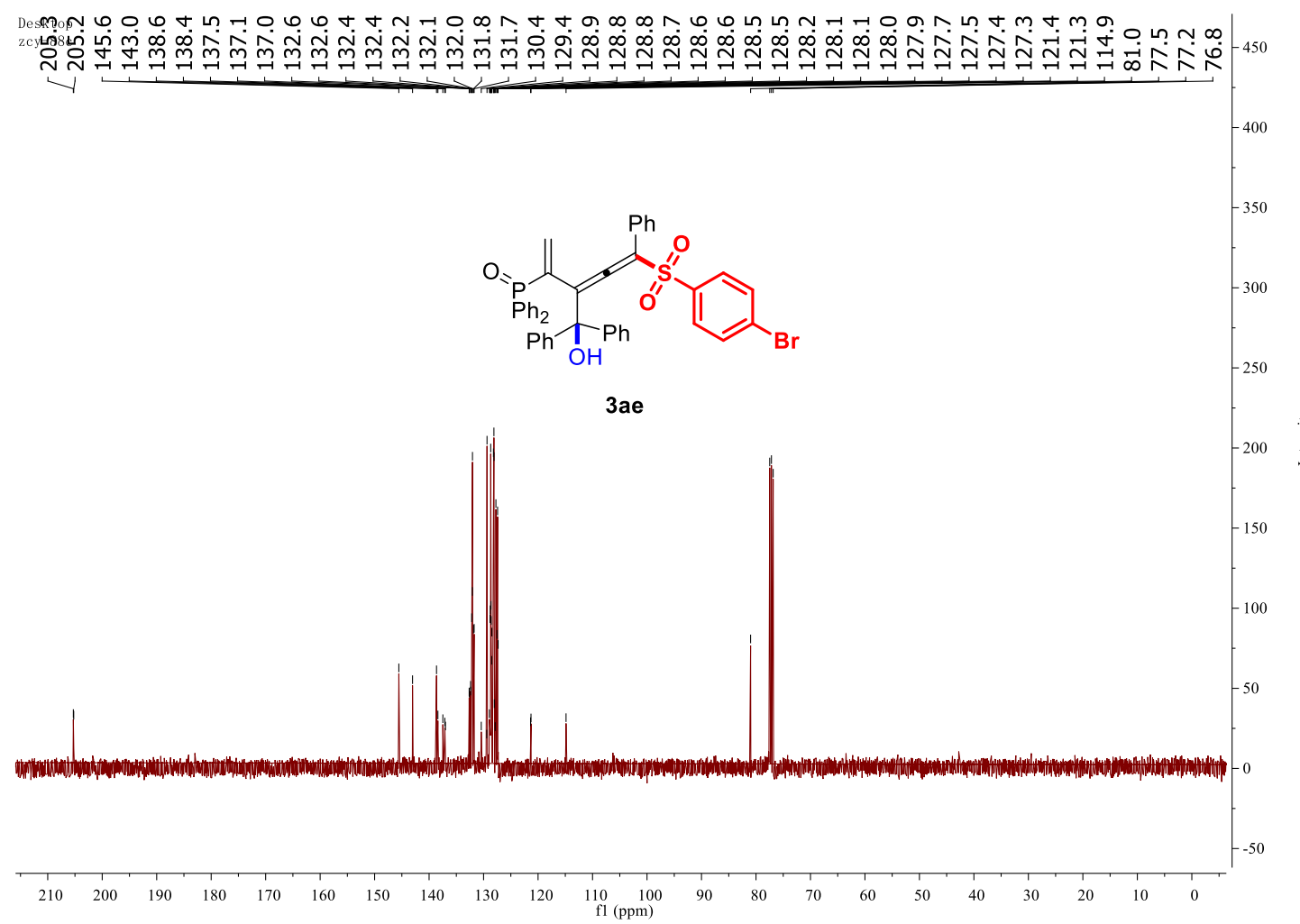

\section{${ }^{13} \mathrm{C}$ NMR $\left(101 \mathrm{MHz}, \mathrm{CDCl}_{3}\right)$ of 3ae}

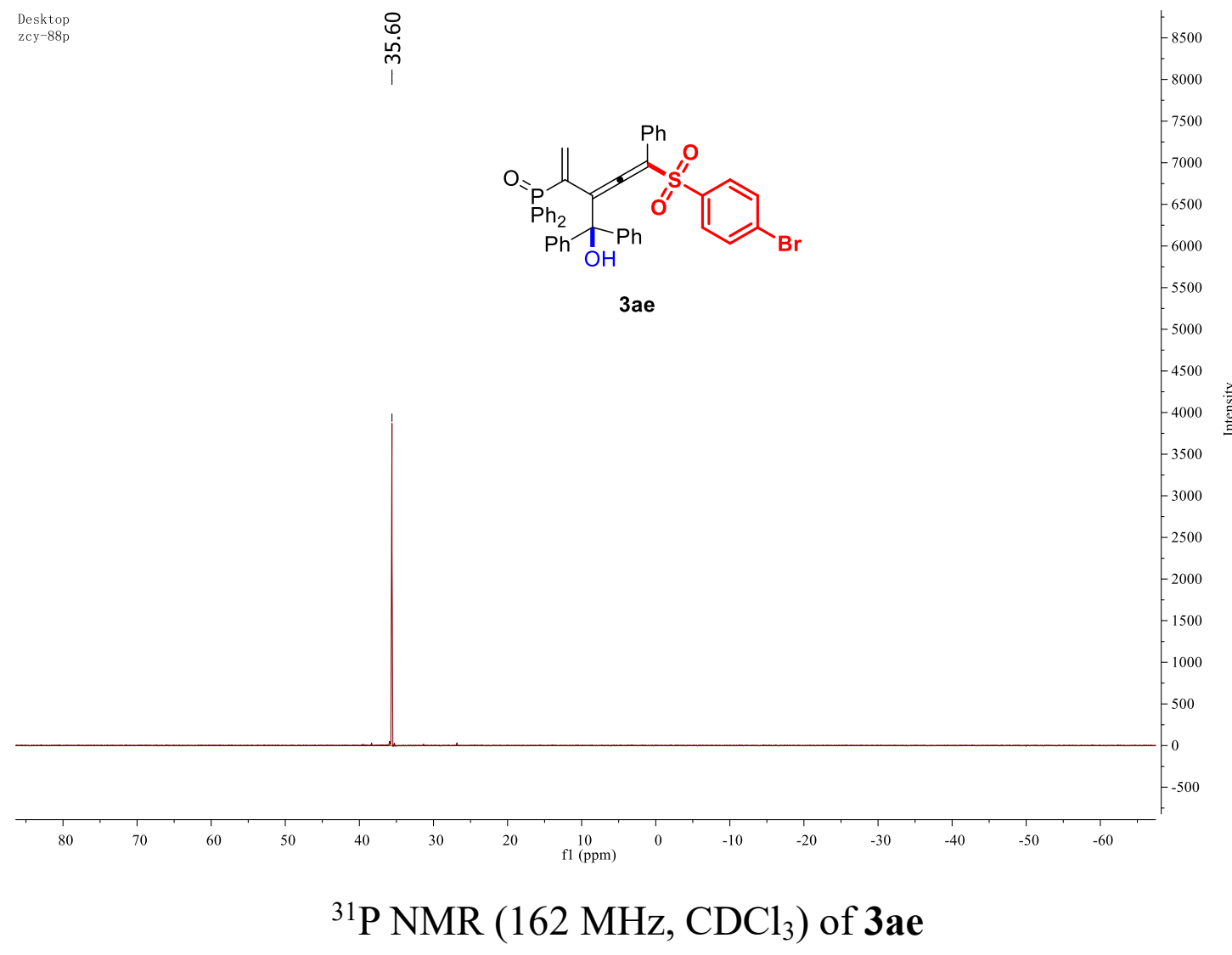


20190506-22 \#25 RT: 0.39 AV: 1 SB: 21 0.01-0.07, 0.78-1.04 NL: 2.10E4 T: FTMS $\{1,1\}+p$ ESI Full ms [100.00-1000.00]
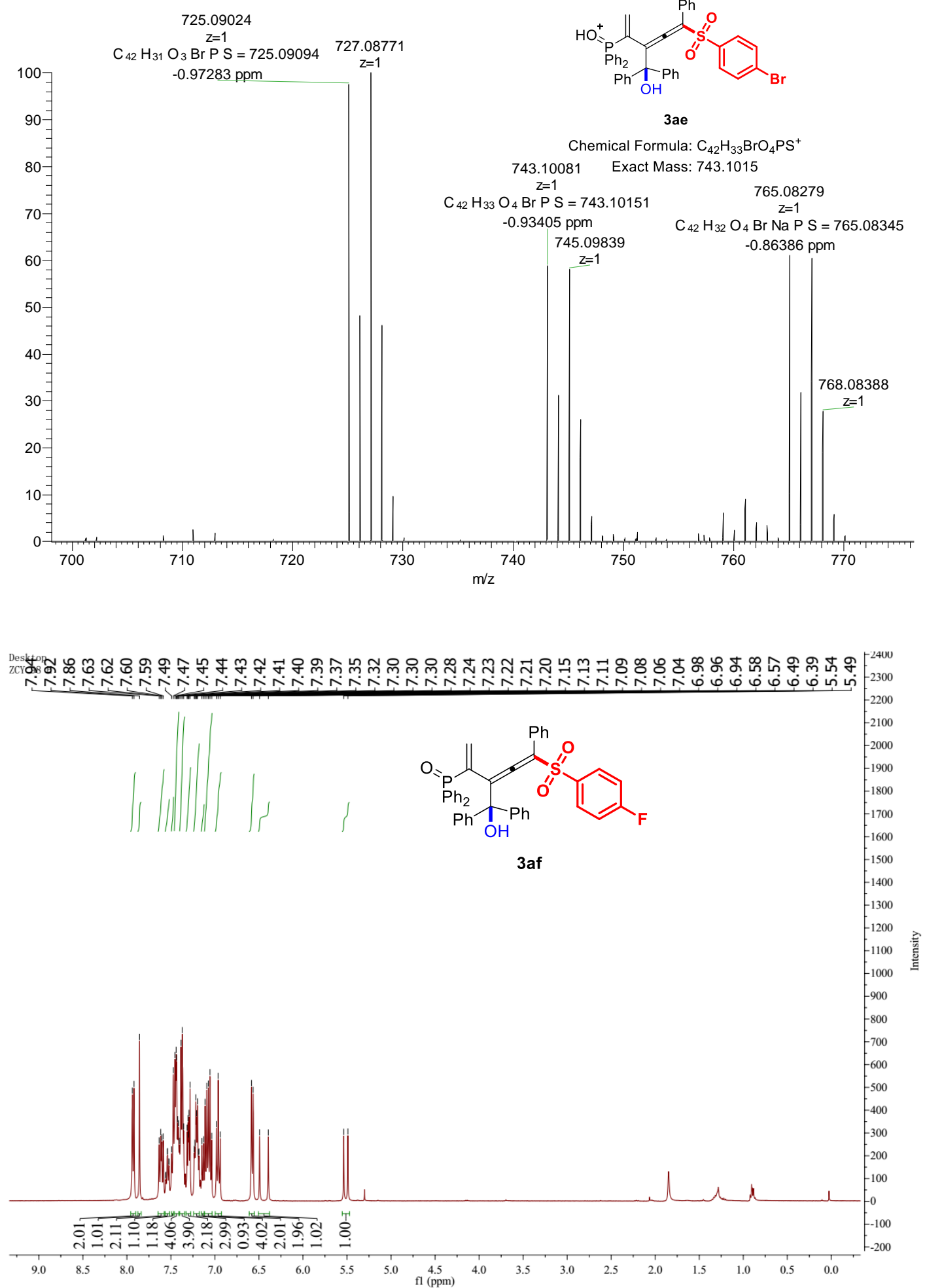

${ }^{1} \mathrm{H} \mathrm{NMR}\left(400 \mathrm{MHz}, \mathrm{CDCl}_{3}\right)$ of $\mathbf{3 a f}$ 


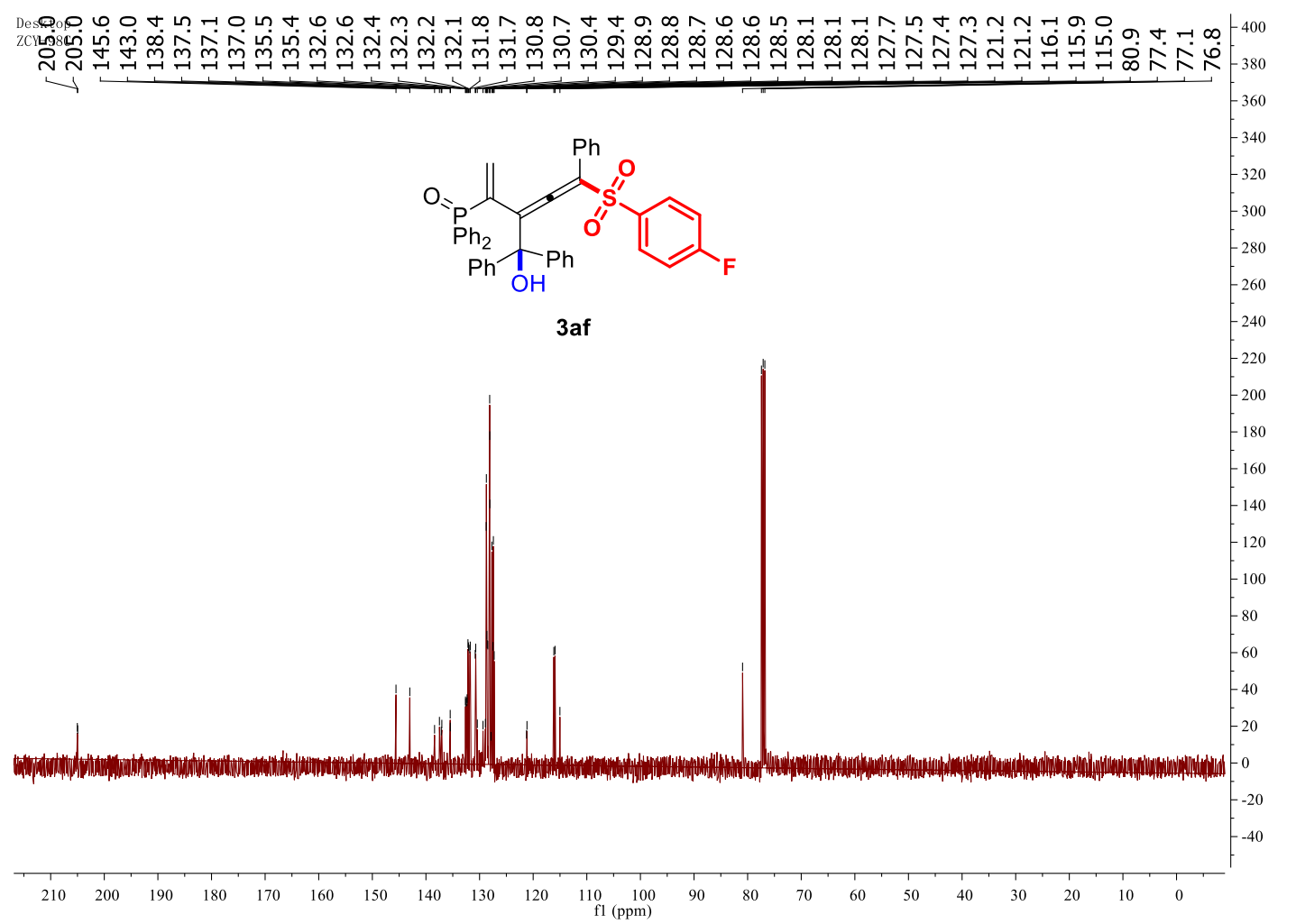

${ }^{13} \mathrm{C} \mathrm{NMR}\left(101 \mathrm{MHz}, \mathrm{CDCl}_{3}\right)$ of $\mathbf{3 a f}$

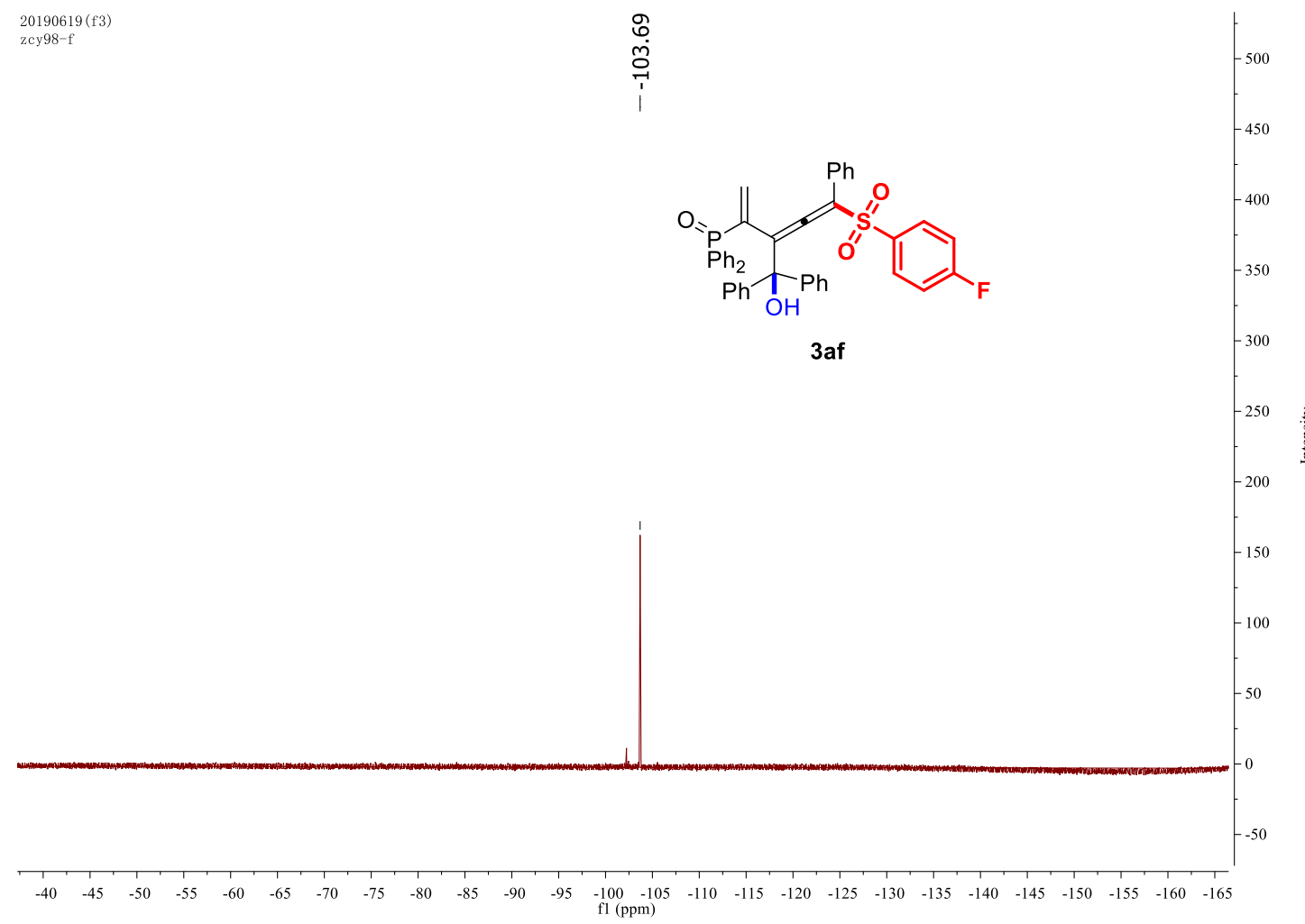

${ }^{19} \mathrm{~F}$ NMR $\left(376 \mathrm{MHz}, \mathrm{CDCl}_{3}\right)$ of $\mathbf{3 a f}$ 


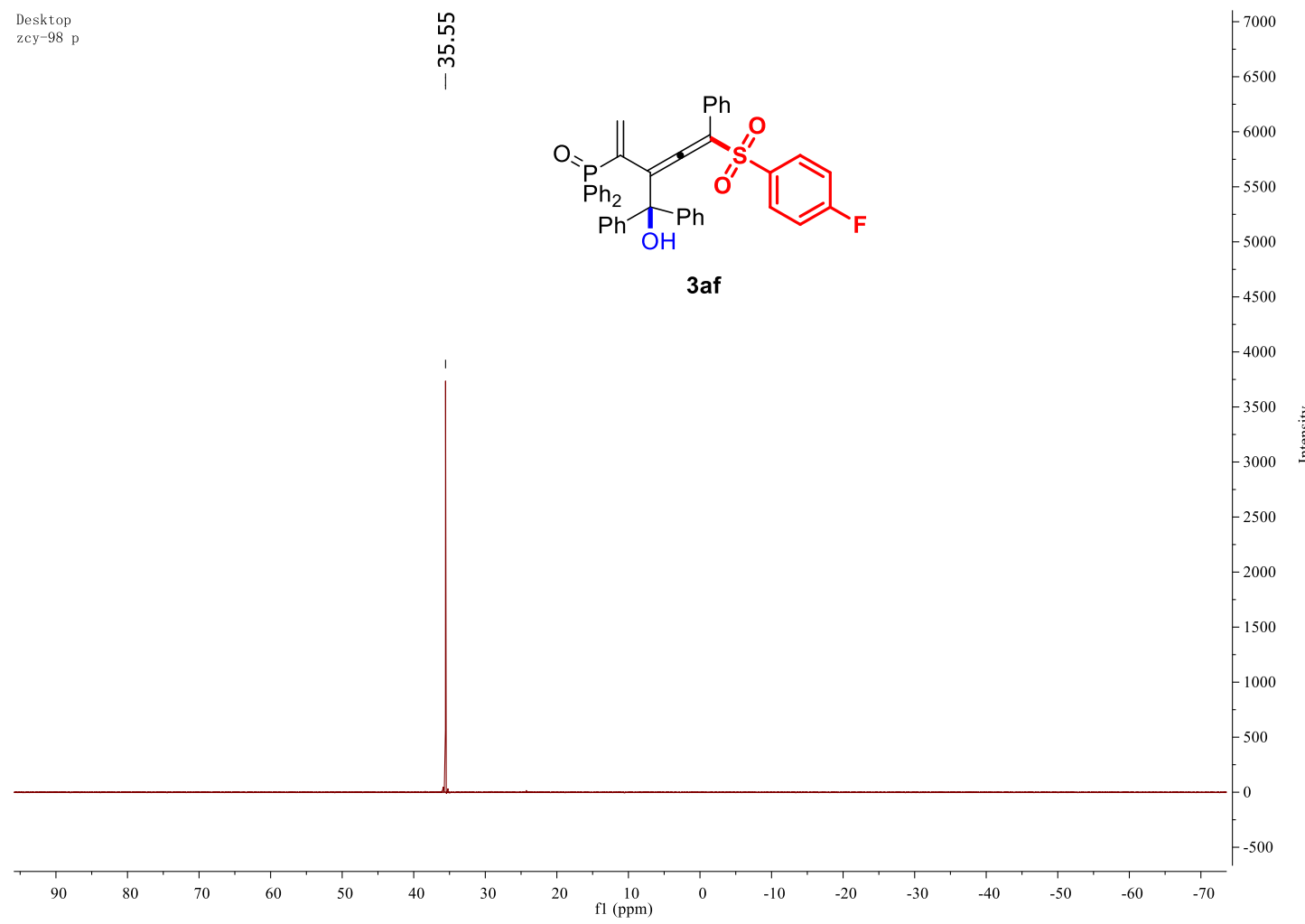

\section{${ }^{31} \mathrm{P}$ NMR (162 MHz, $\mathrm{CDCl}_{3}$ ) of 3af}

20190524-8 \#9 RT: 0.14 AV: 1 SB: 36 0.01-0.04, 0.48-1.04 NL: 3.14E6 T: FTMS $\{1,1\}+p$ ESI Full ms [100.00-1000.00] 665.17198

$\mathrm{C}_{42} \mathrm{H}_{31} \mathrm{O}_{3} \mathrm{FP} \mathrm{S}=665.17101$

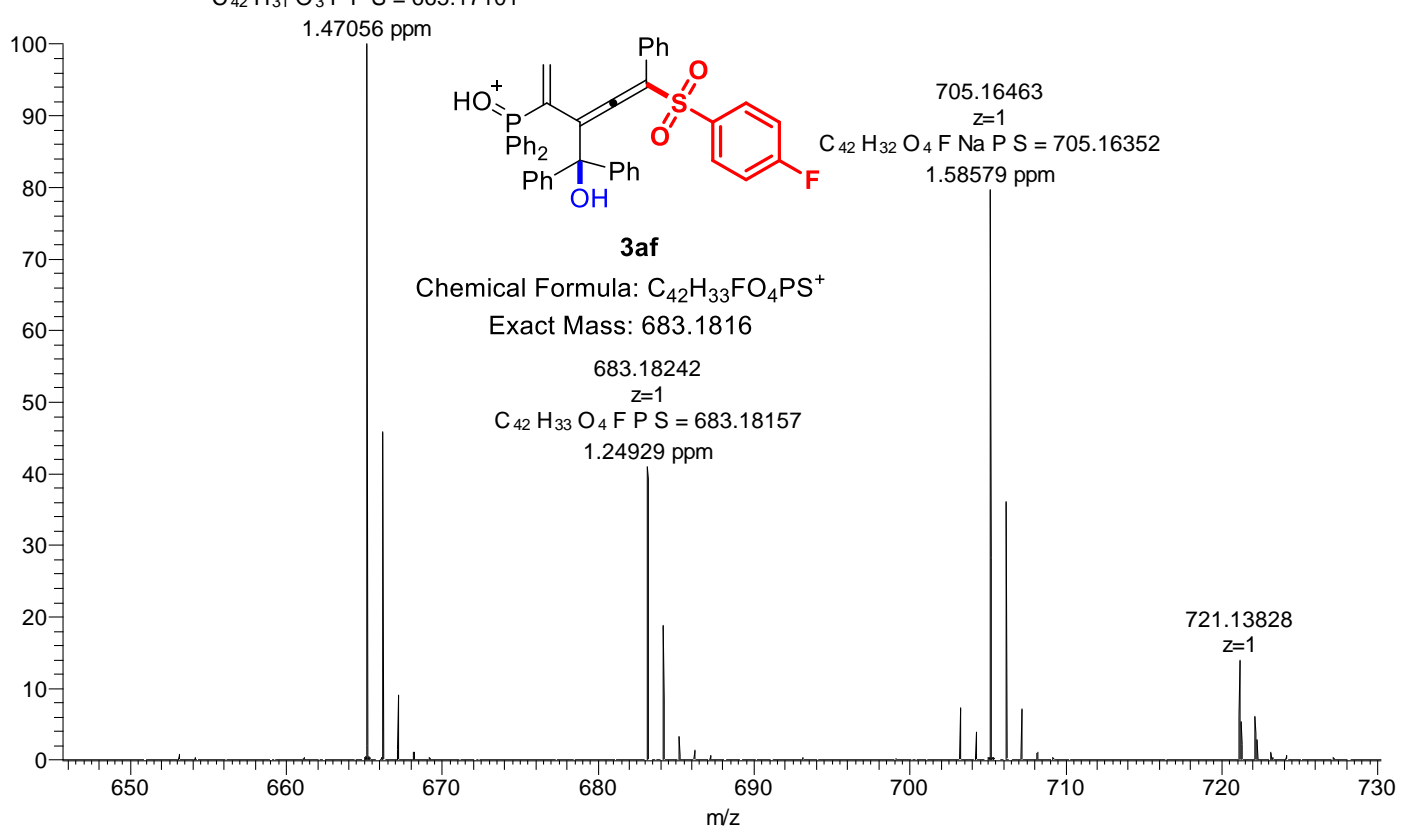



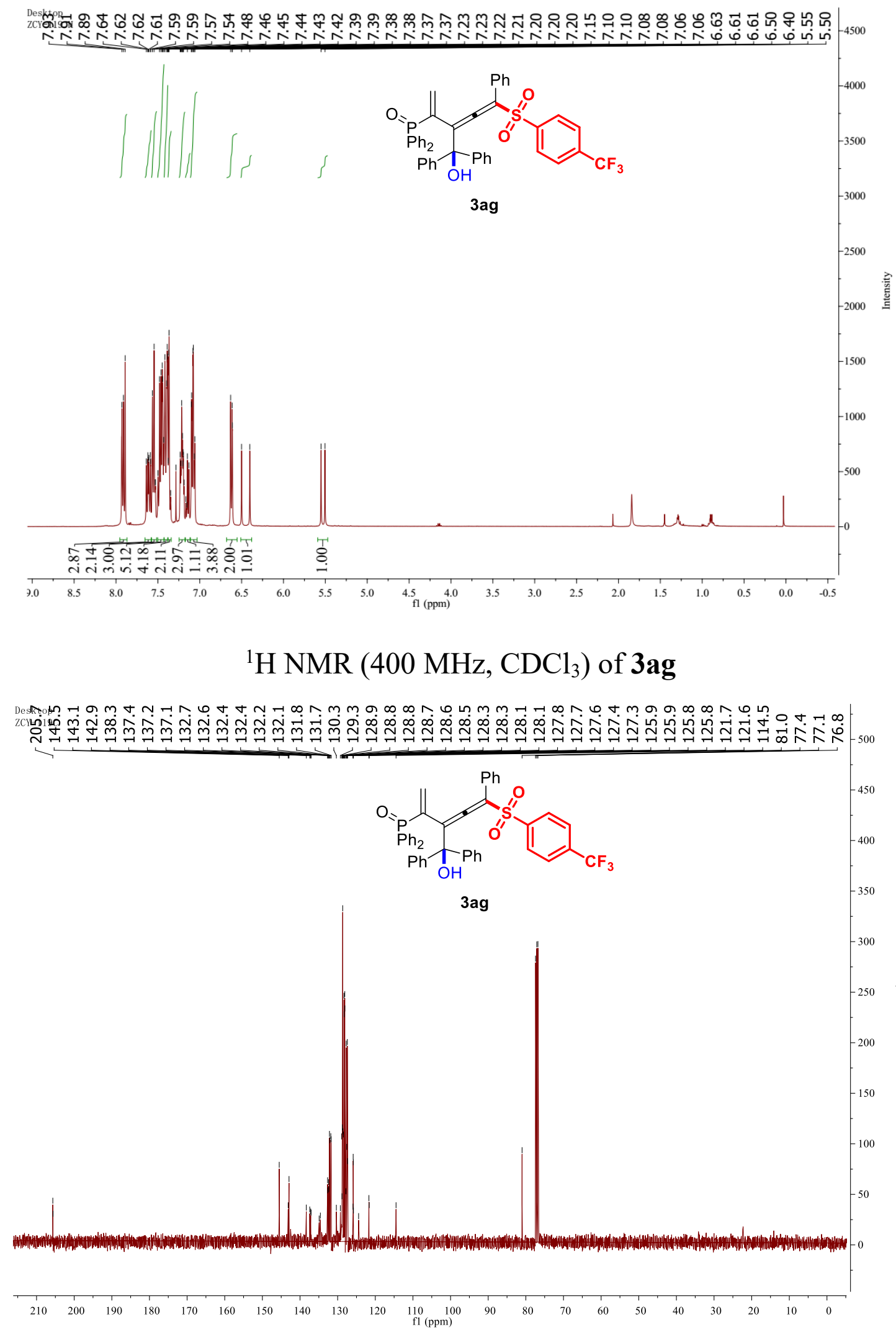

${ }^{13} \mathrm{C}$ NMR $\left(101 \mathrm{MHz}, \mathrm{CDCl}_{3}\right)$ of $\mathbf{3 a g}$ 


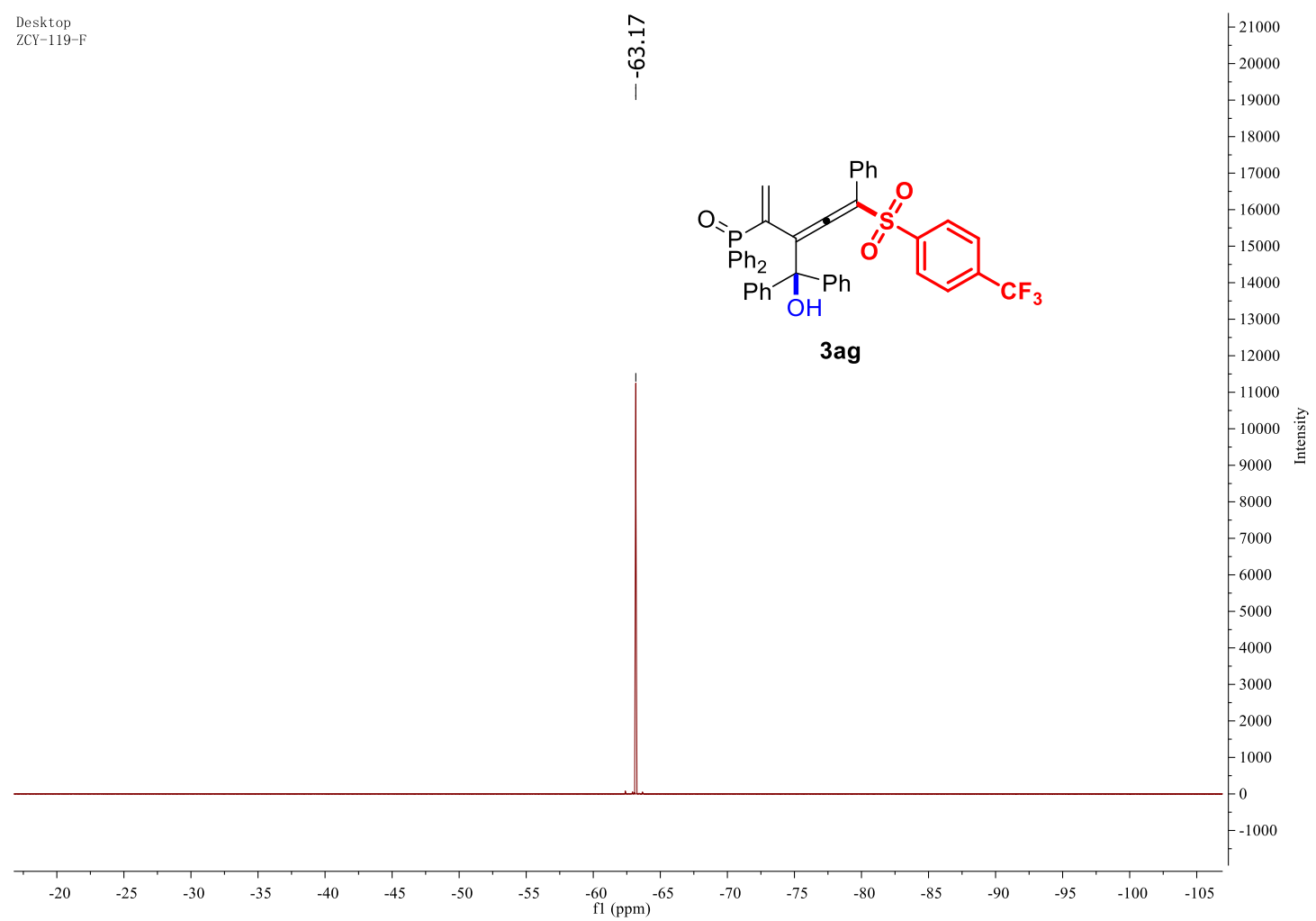

\section{${ }^{19} \mathrm{~F}$ NMR $\left(376 \mathrm{MHz}, \mathrm{CDCl}_{3}\right)$ of $\mathbf{3 a g}$}

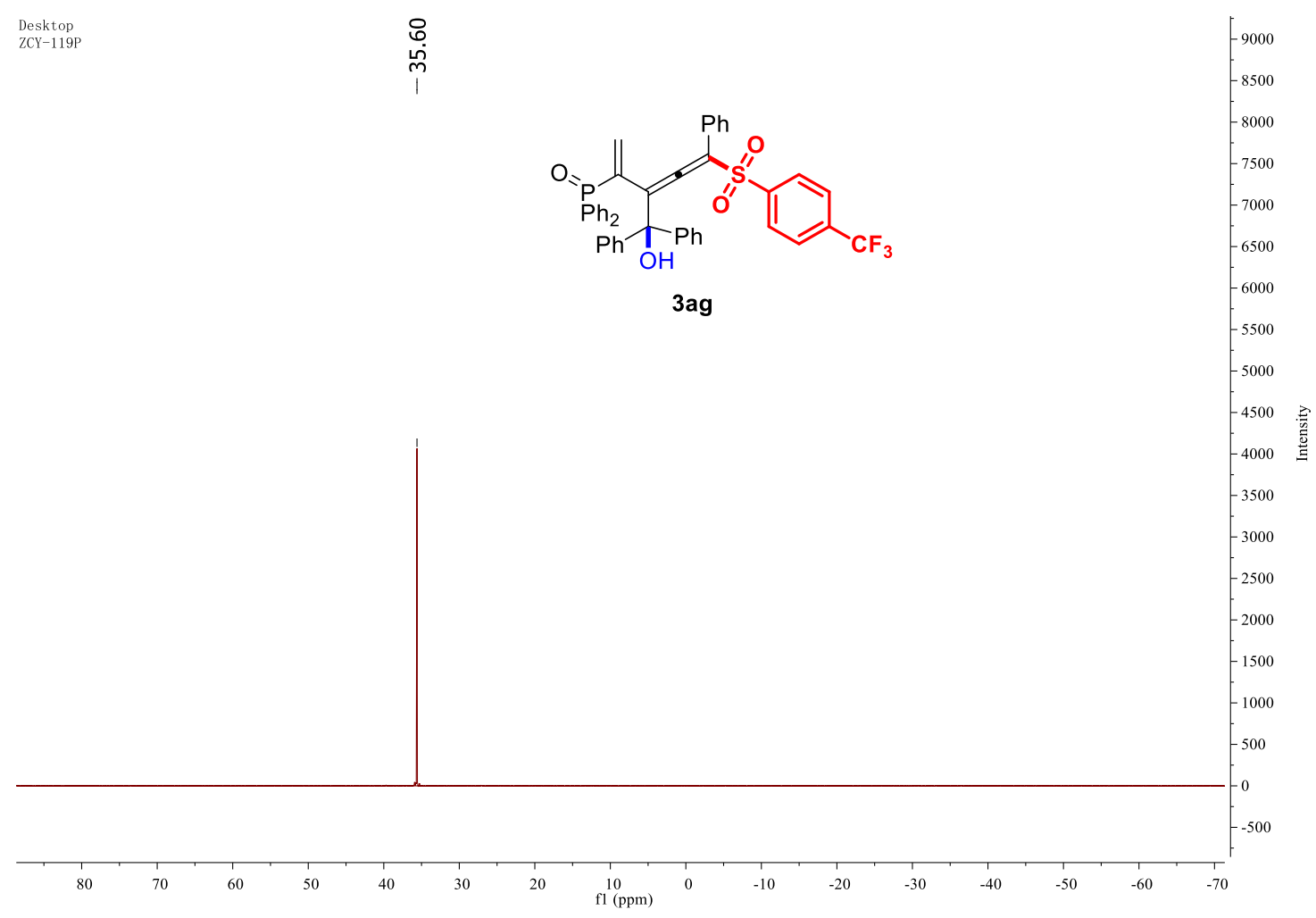

${ }^{31} \mathrm{P}$ NMR $\left(162 \mathrm{MHz}, \mathrm{CDCl}_{3}\right)$ of $\mathbf{3 a g}$ 

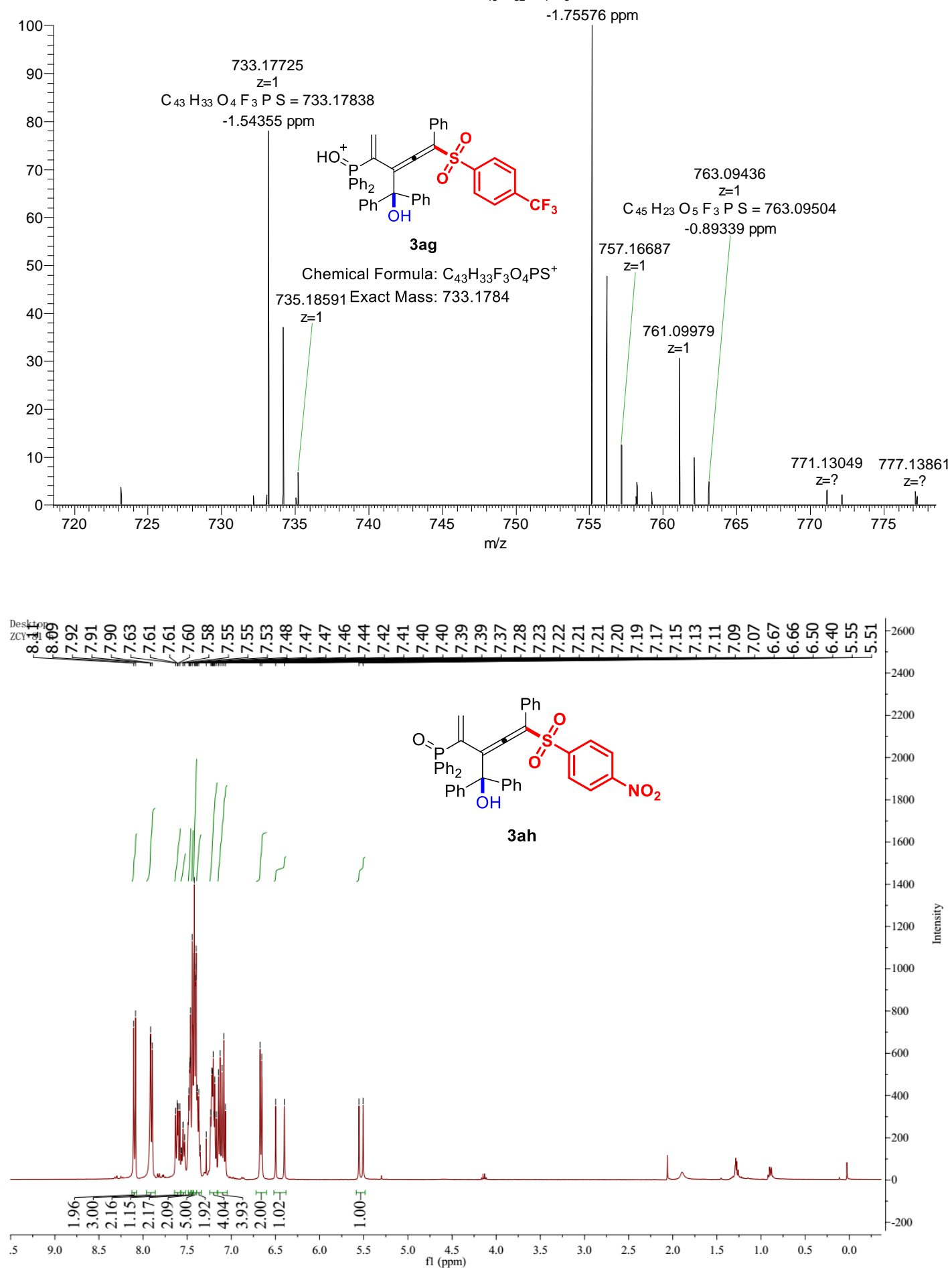

${ }^{1} \mathrm{H}$ NMR $\left(400 \mathrm{MHz}, \mathrm{CDCl}_{3}\right)$ of $\mathbf{3 a h}$ 


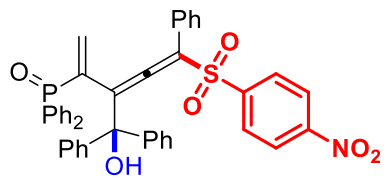

$3 a h$

${ }^{13} \mathrm{C}$ NMR $\left(101 \mathrm{MHz}, \mathrm{CDCl}_{3}\right)$ of $\mathbf{3 a h}$

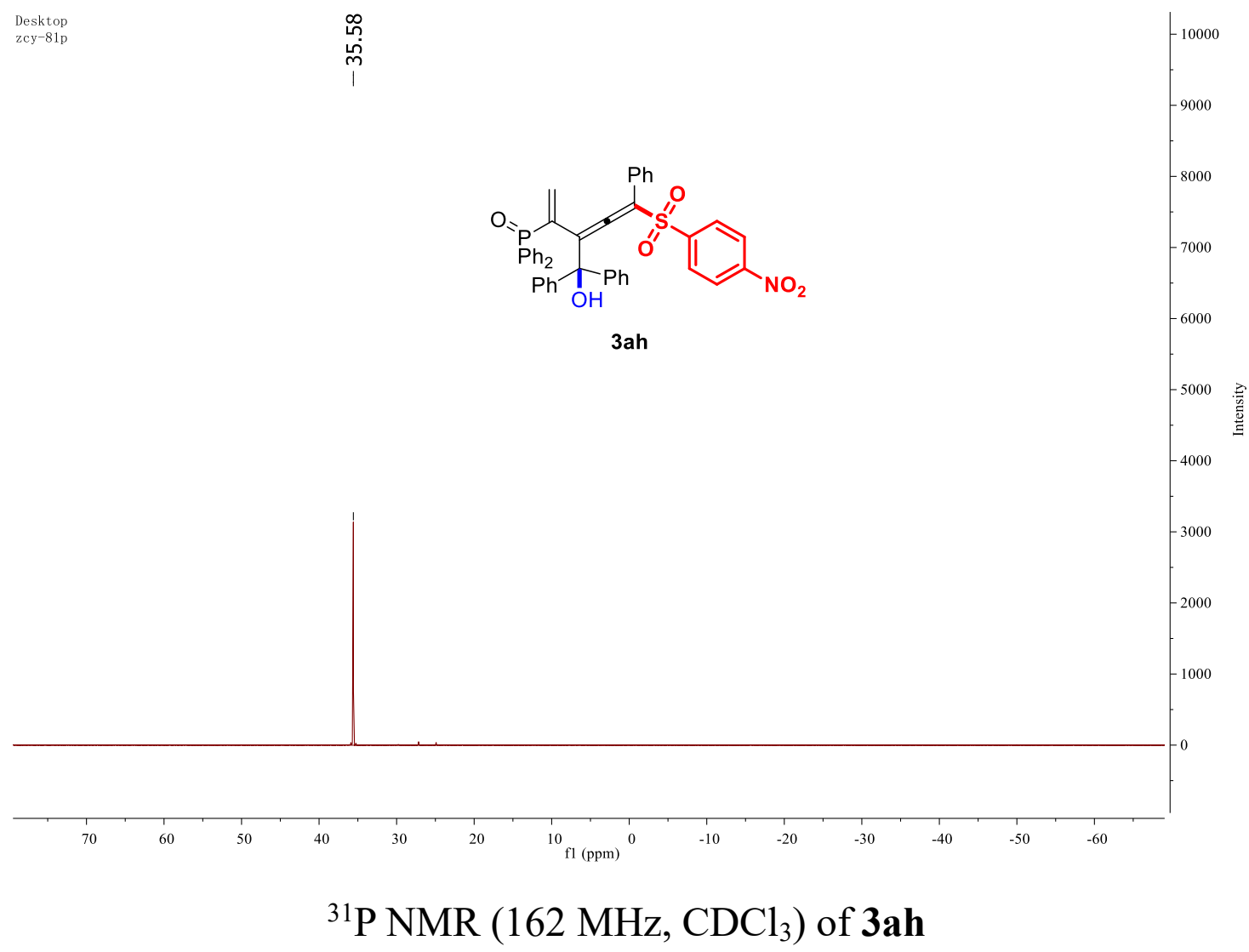


20190506-20 \#15 RT: 0.22 AV: 1 SB: 21 0.01-0.07, 0.78-1.04 NL: 2.64E6

T: FTMS $\{1,1\}+p$ ESI Full ms [100.00-1000.00]

528.10249

$\mathrm{Z}=1$

$\mathrm{C}_{29} \mathrm{H}_{23} \mathrm{O}_{5} \mathrm{~N} \mathrm{P} \mathrm{S}=528.10291$
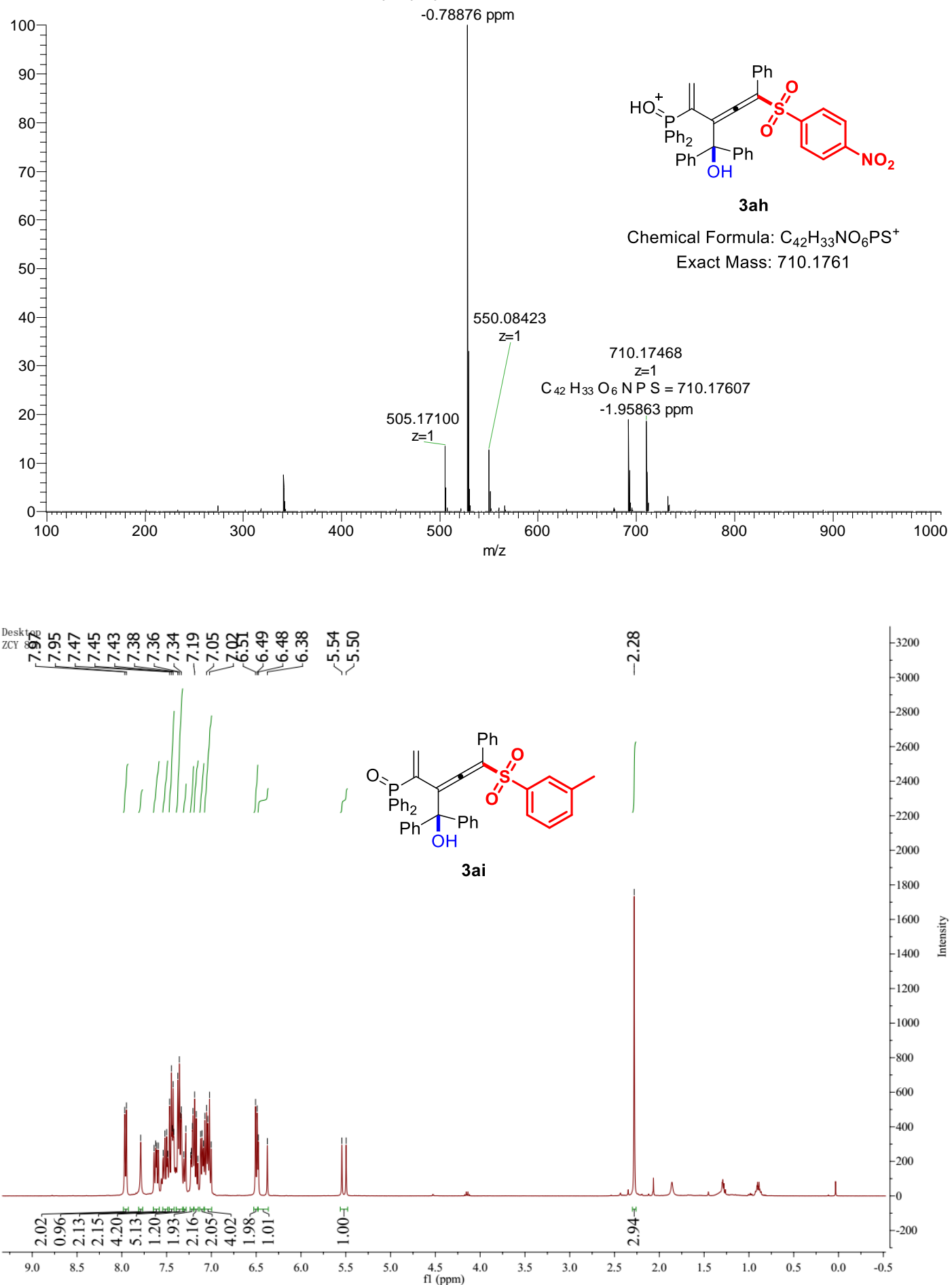

${ }^{1} \mathrm{H}$ NMR (400 MHz, $\mathrm{CDCl}_{3}$ ) of 3ai 


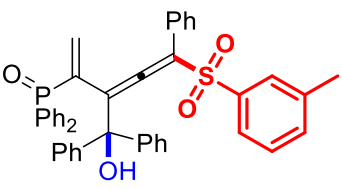

$3 a i$

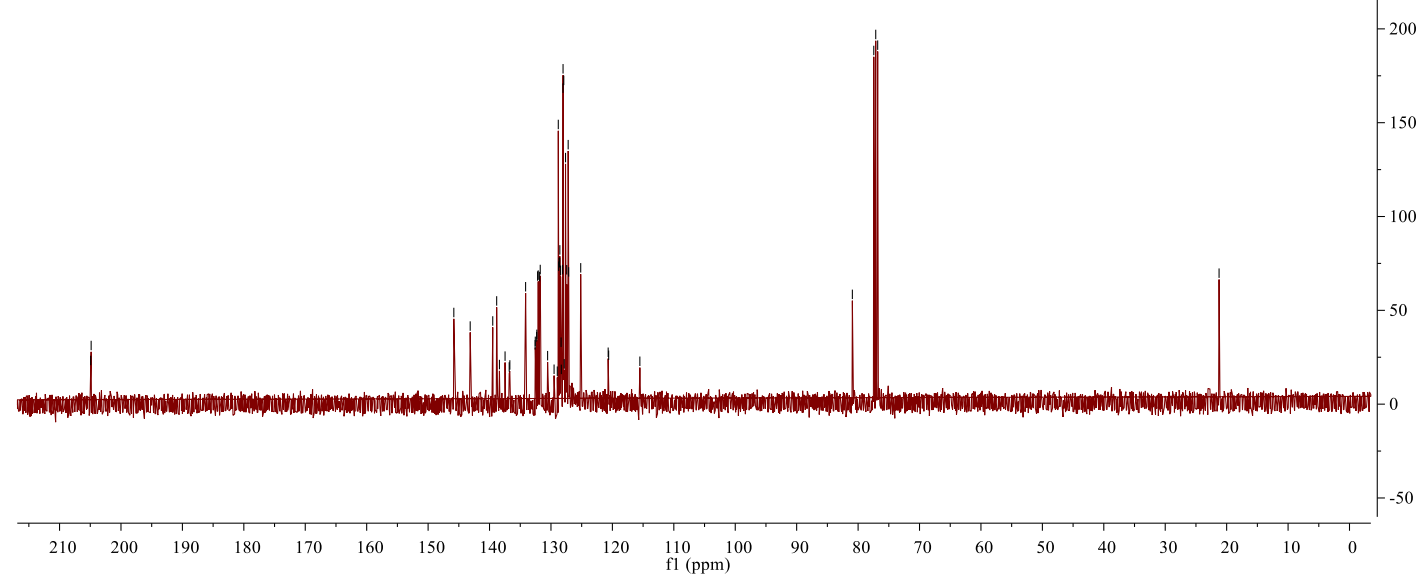

${ }^{13} \mathrm{C} \mathrm{NMR}\left(101 \mathrm{MHz}, \mathrm{CDCl}_{3}\right)$ of 3ai

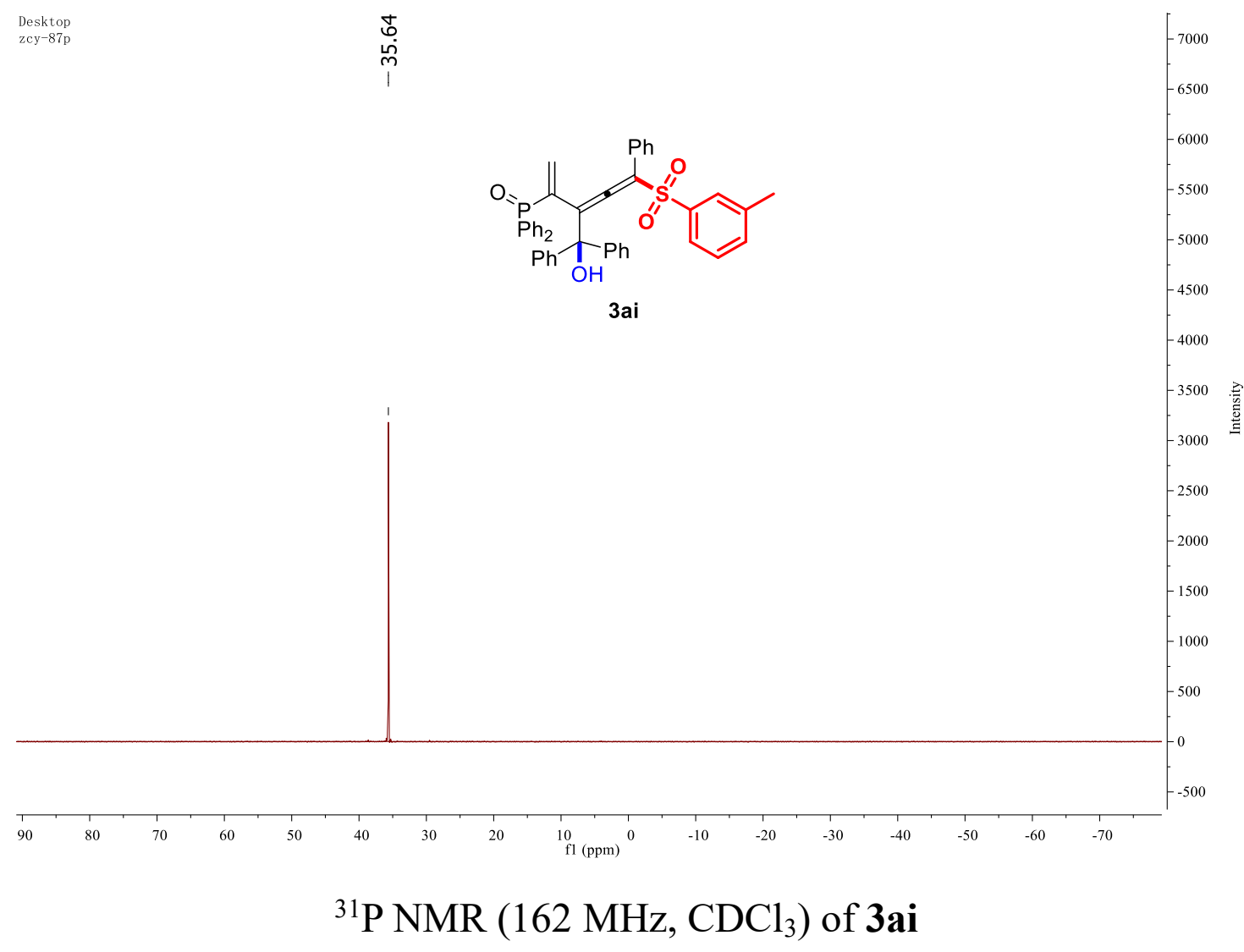


20190506-21 \#19 RT: 0.29 AV: 1 SB: 21 0.01-0.07, 0.77-1.03 NL: 4.05E5 T: FTMS $\{1,1\}+p$ ESI Full ms [100.00-1000.00] 661.19549
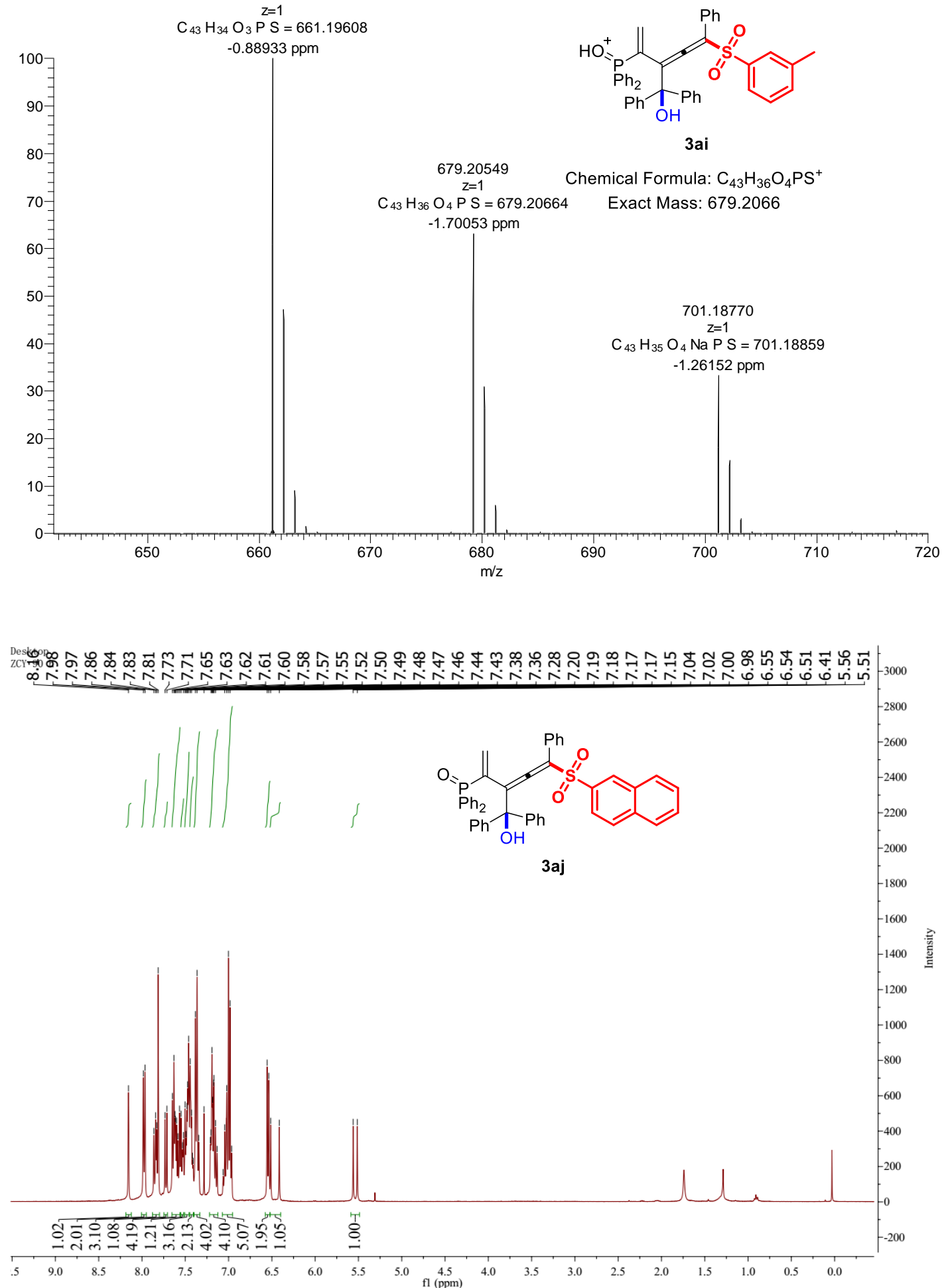

${ }^{1} \mathrm{H}$ NMR (400 MHz, $\mathrm{CDCl}_{3}$ ) of $\mathbf{3 a j}$ 


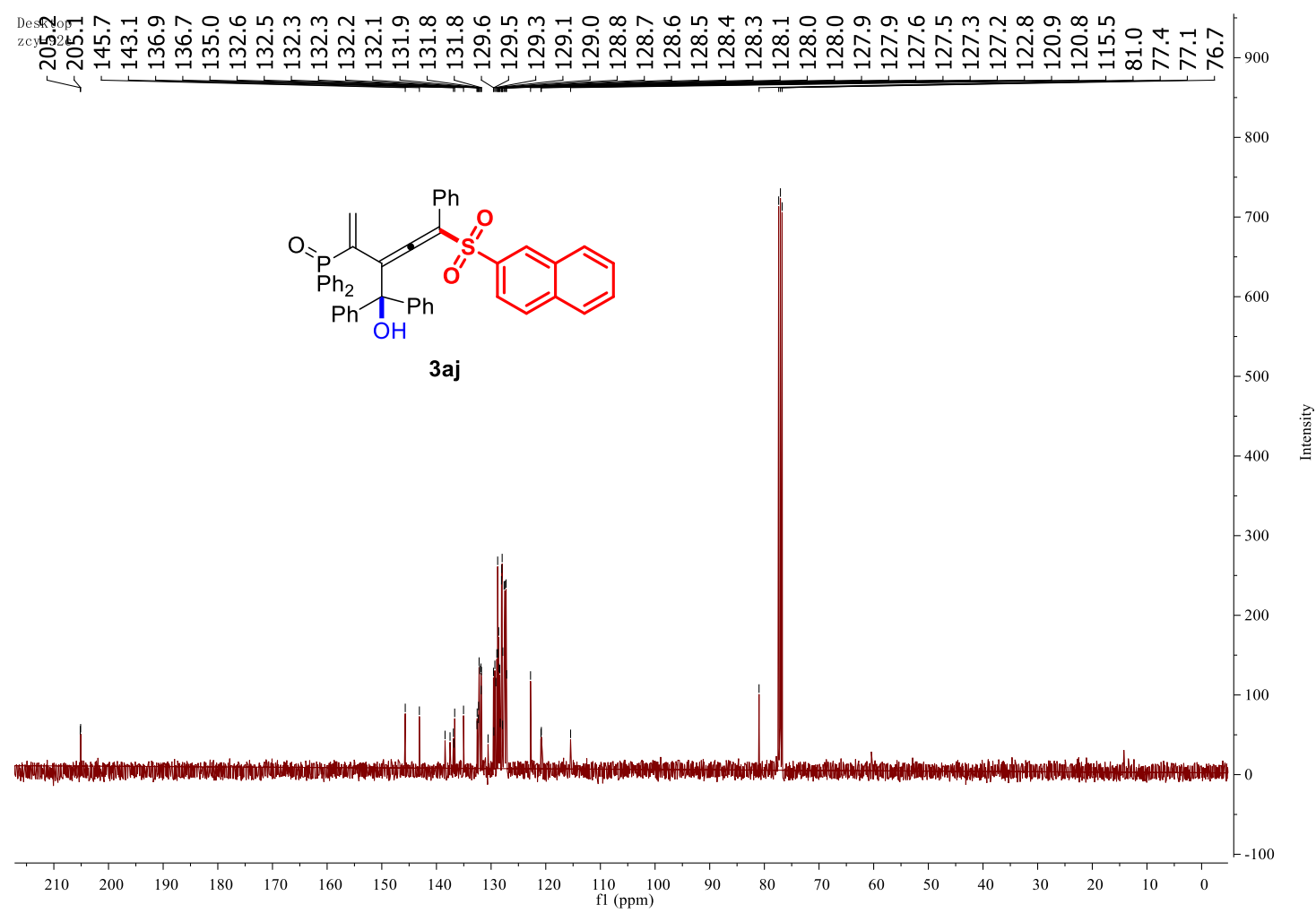

${ }^{13} \mathrm{C}$ NMR $\left(101 \mathrm{MHz}, \mathrm{CDCl}_{3}\right)$ of $\mathbf{3 a j}$

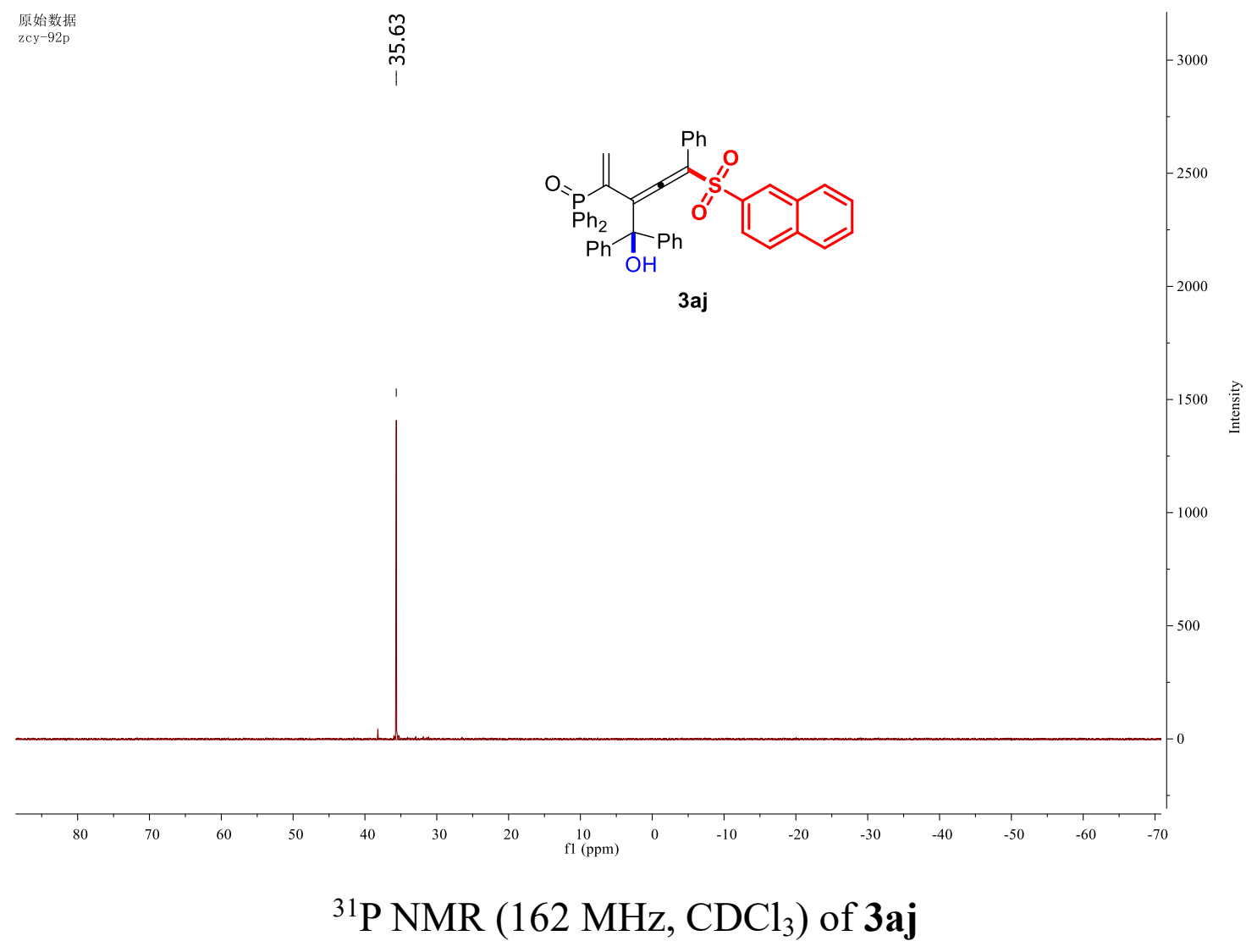


20190506-23 \#21 RT: 0.33 AV: 1 SB: 21 0.01-0.07, 0.78-1.04 NL: 7.84E4 T: FTMS $\{1,1\}+p$ ESI Full ms [100.00-1000.00]

$$
z=1
$$
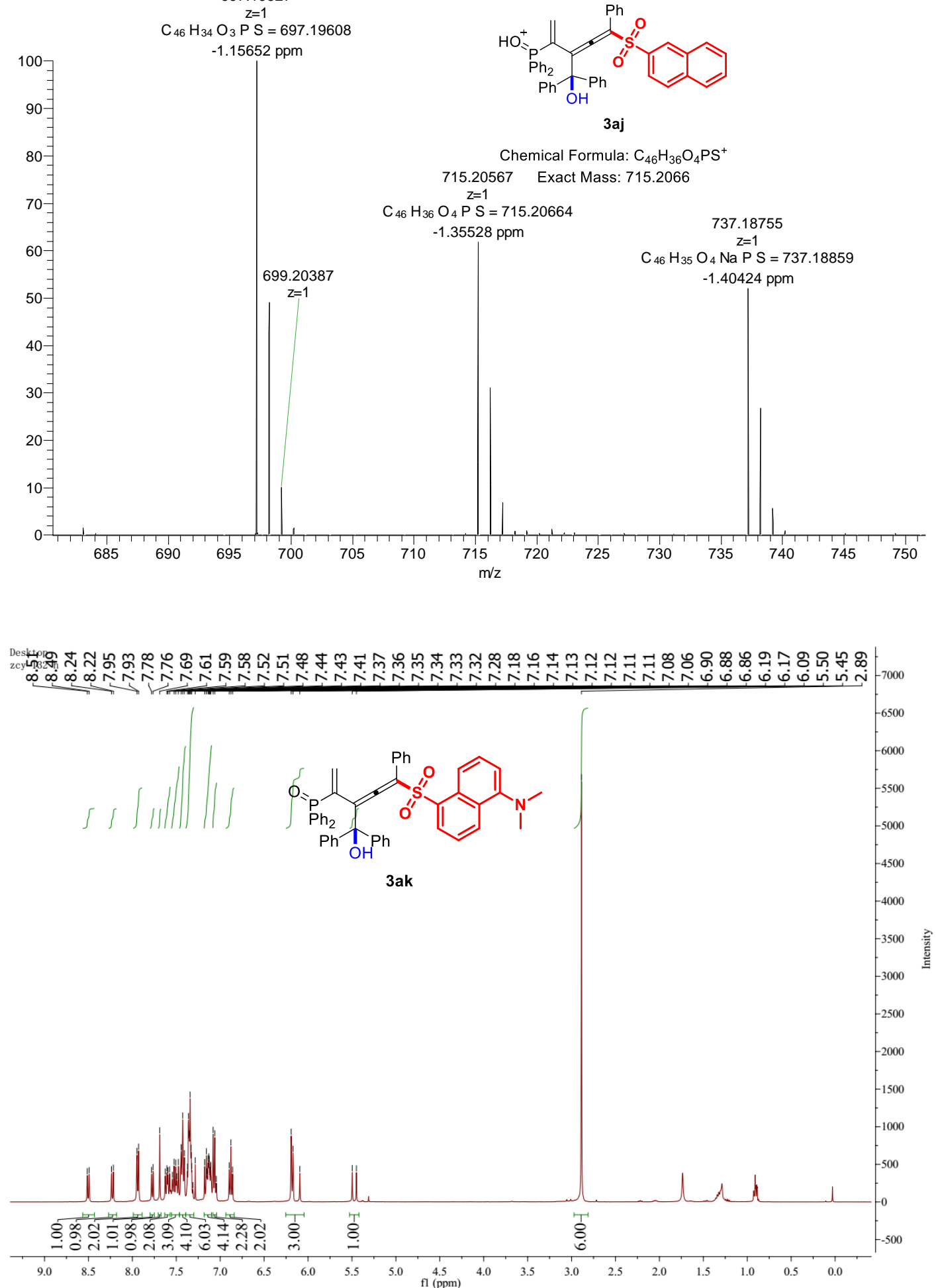

${ }^{1} \mathrm{H}$ NMR $\left(400 \mathrm{MHz}, \mathrm{CDCl}_{3}\right)$ of $\mathbf{3 a k}$ 


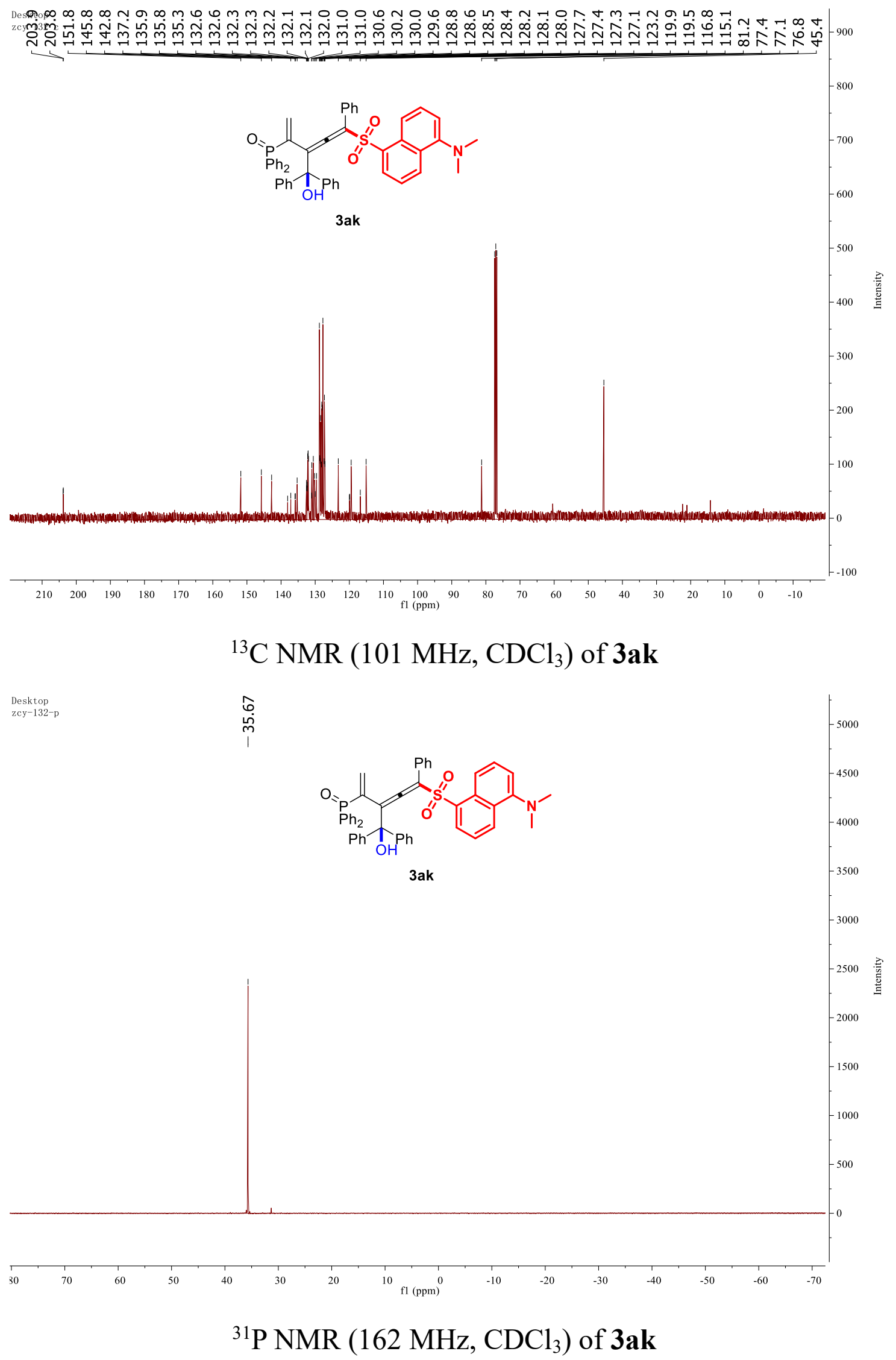


11 \#7 RT: 0.11 AV: 1 NL: 9.12E4

T: FTMS $\{1,1\}+p$ ESI Full ms [100.00-1000.00]
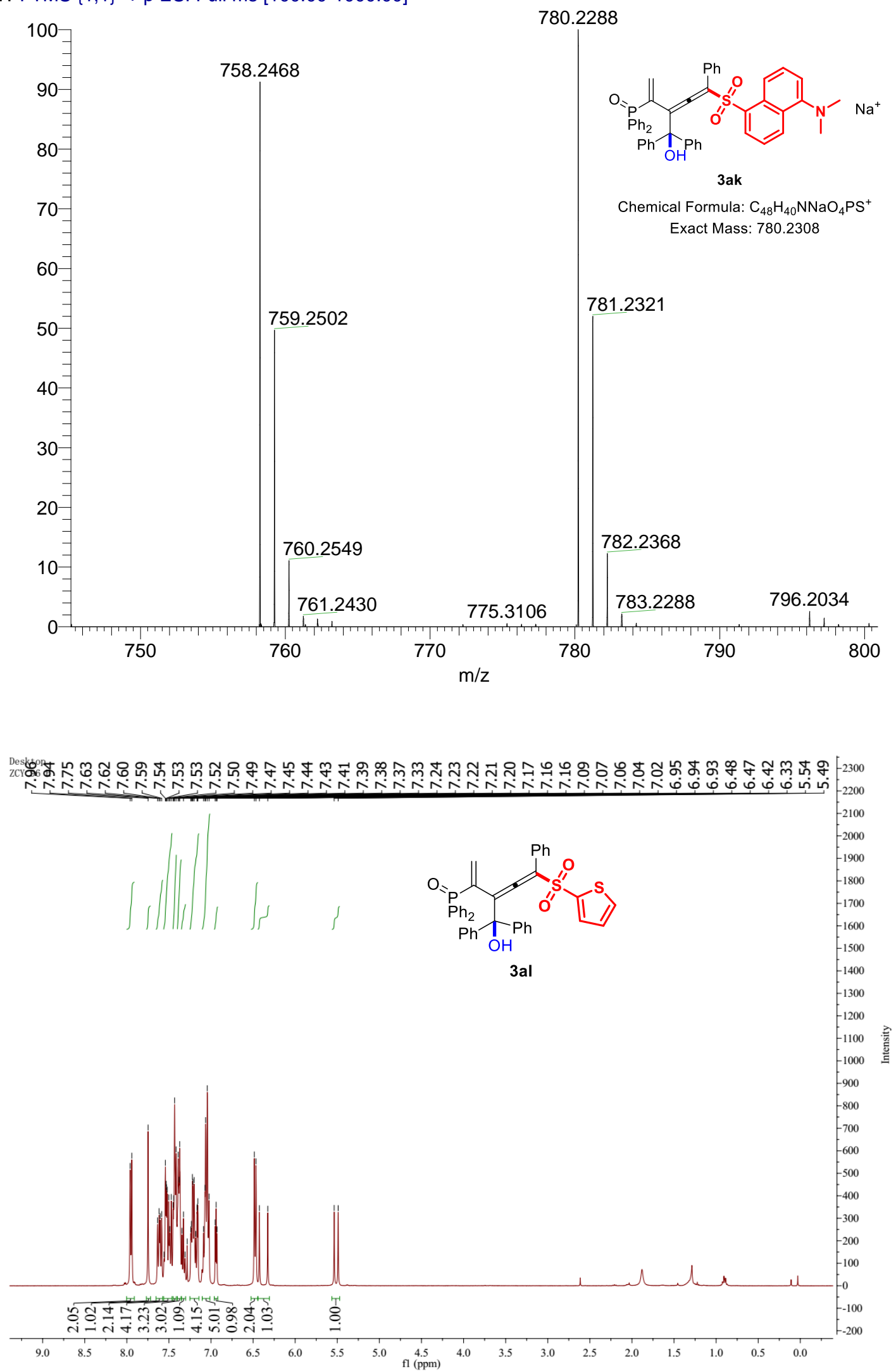

${ }^{1} \mathrm{H} \mathrm{NMR}\left(400 \mathrm{MHz}, \mathrm{CDCl}_{3}\right)$ of $\mathbf{3 a l}$ 

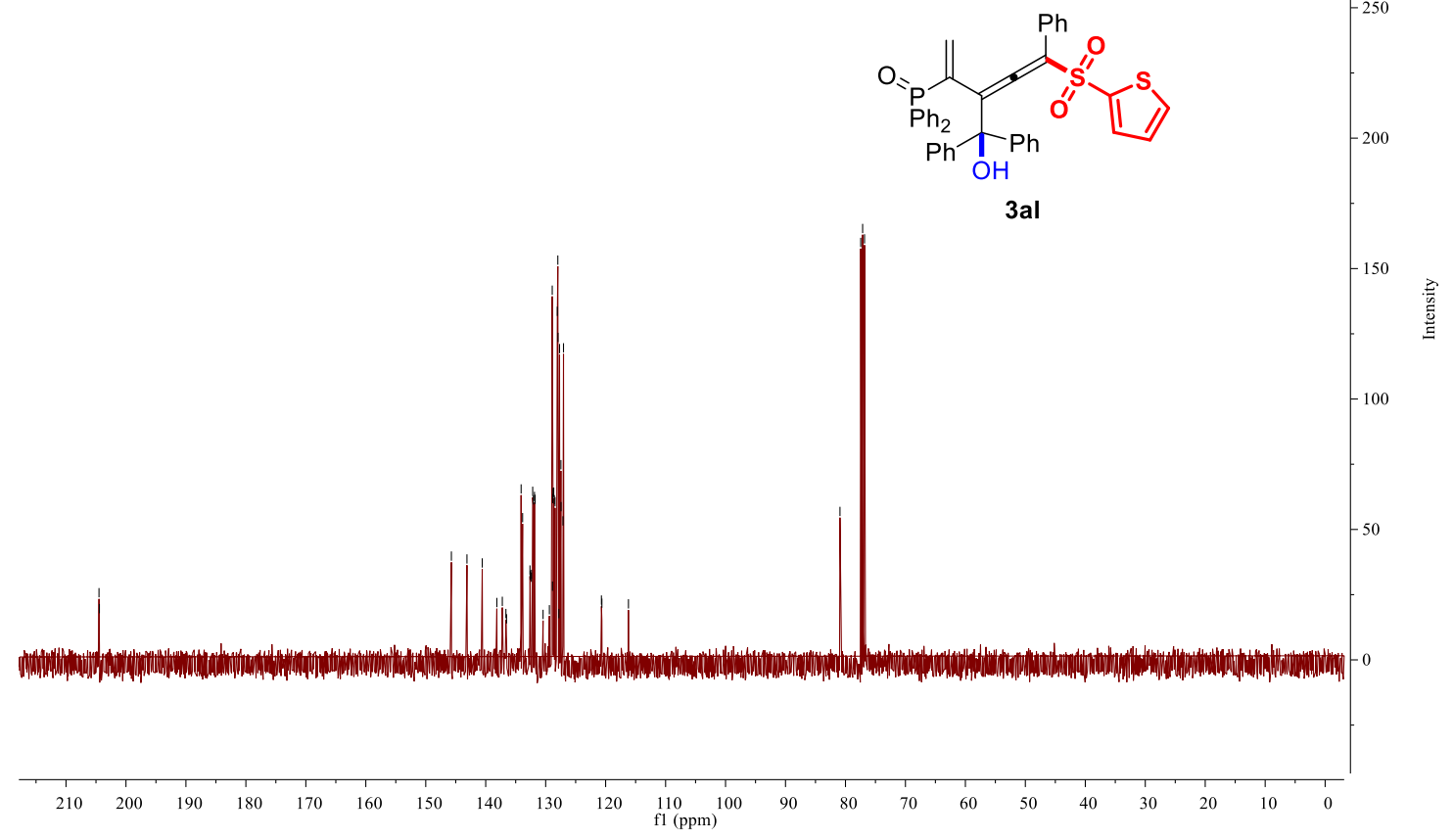

${ }^{13} \mathrm{C} \mathrm{NMR}\left(101 \mathrm{MHz}, \mathrm{CDCl}_{3}\right)$ of $\mathbf{3 a l}$

$=$

กี่

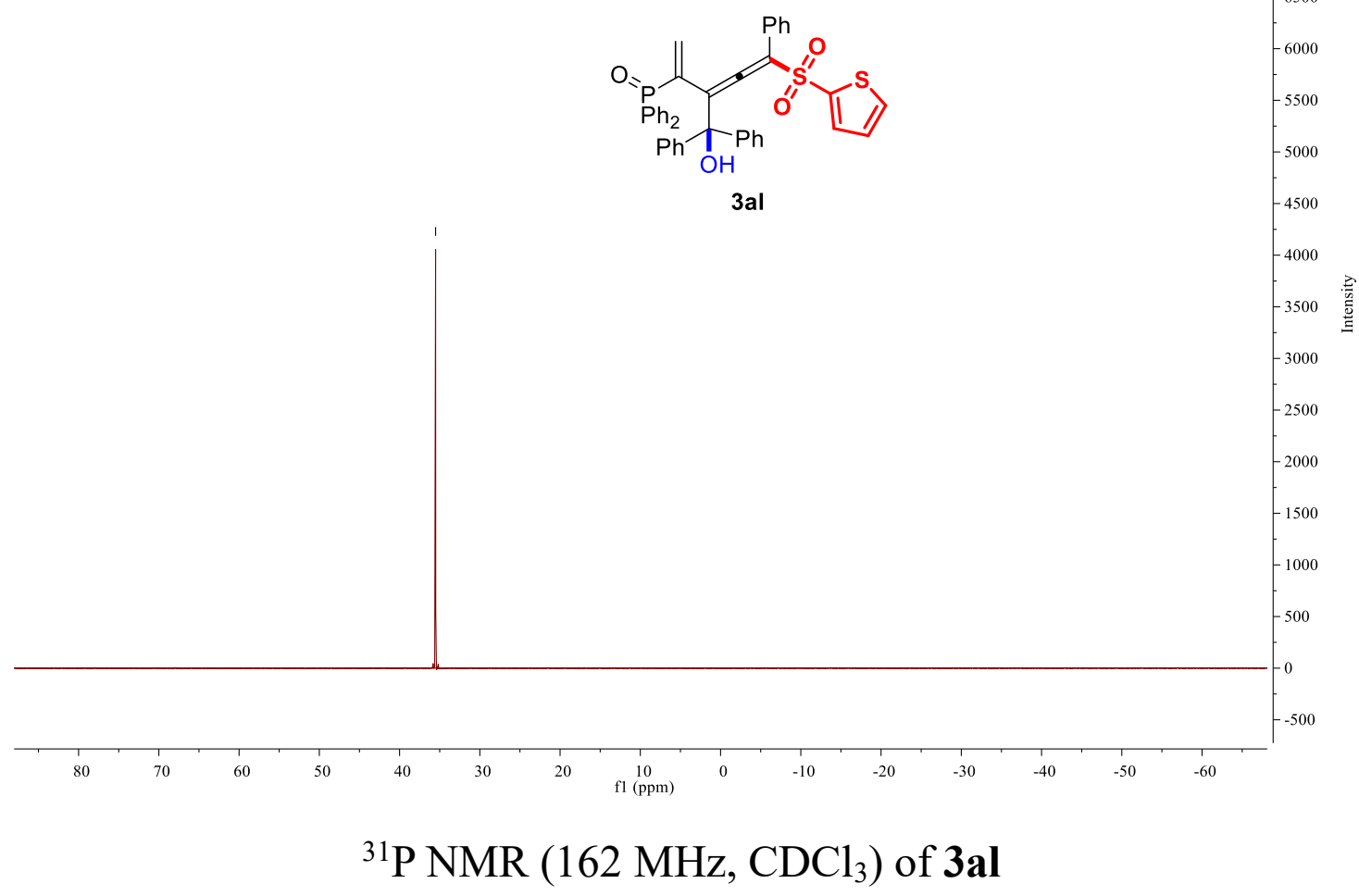


20190524-6 \#11 RT: 0.16 AV: 1 SB: 36 0.01-0.04, 0.46-1.02 NL: 1.37E7

T: FTMS $\{1,1\}+p$ ESI Full ms [100.00-1000.00]

653.13786

$\mathrm{C}_{40} \mathrm{H}_{30} \mathrm{O}_{3} \mathrm{P} \mathrm{S}_{2}=653.13685$

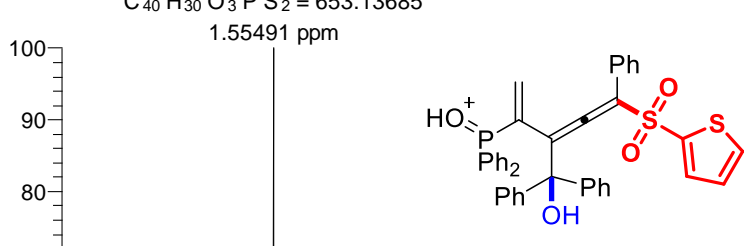

3al 693.13018

Chemical Formula: $\mathrm{C}_{40} \mathrm{H}_{32} \mathrm{O}_{4} \mathrm{PS}_{2}{ }^{+} \quad \begin{gathered}\mathrm{Z}=1 \\ \mathrm{C}_{40} \mathrm{H}_{31} \mathrm{O}_{4} \mathrm{Na} \mathrm{P} \mathrm{S}\end{gathered}$

Exact Mass: $671.1474 \quad 1.19186 \mathrm{ppm}$
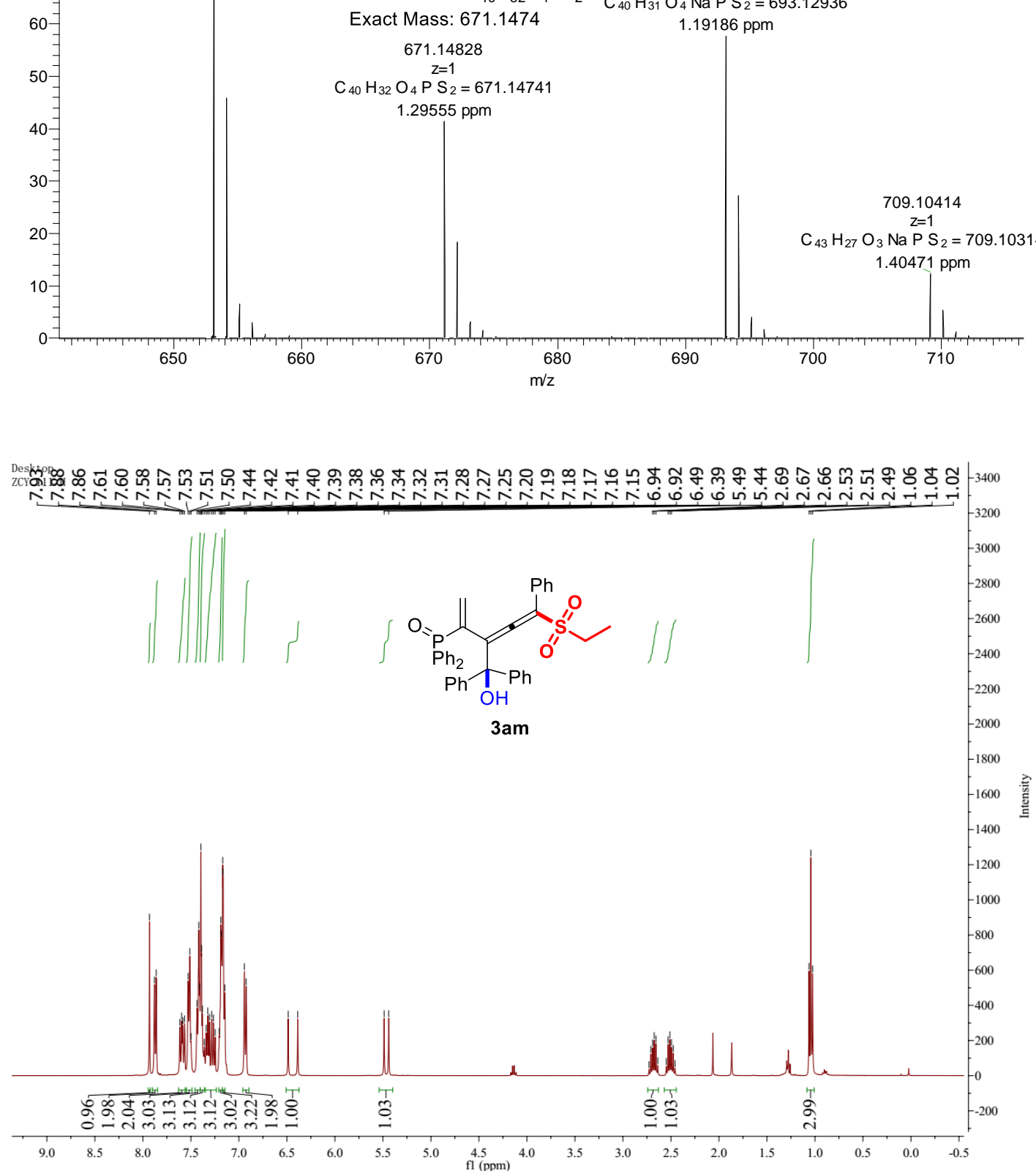

${ }^{1} \mathrm{H}$ NMR $\left(400 \mathrm{MHz}, \mathrm{CDCl}_{3}\right)$ of $\mathbf{3 a m}$ 


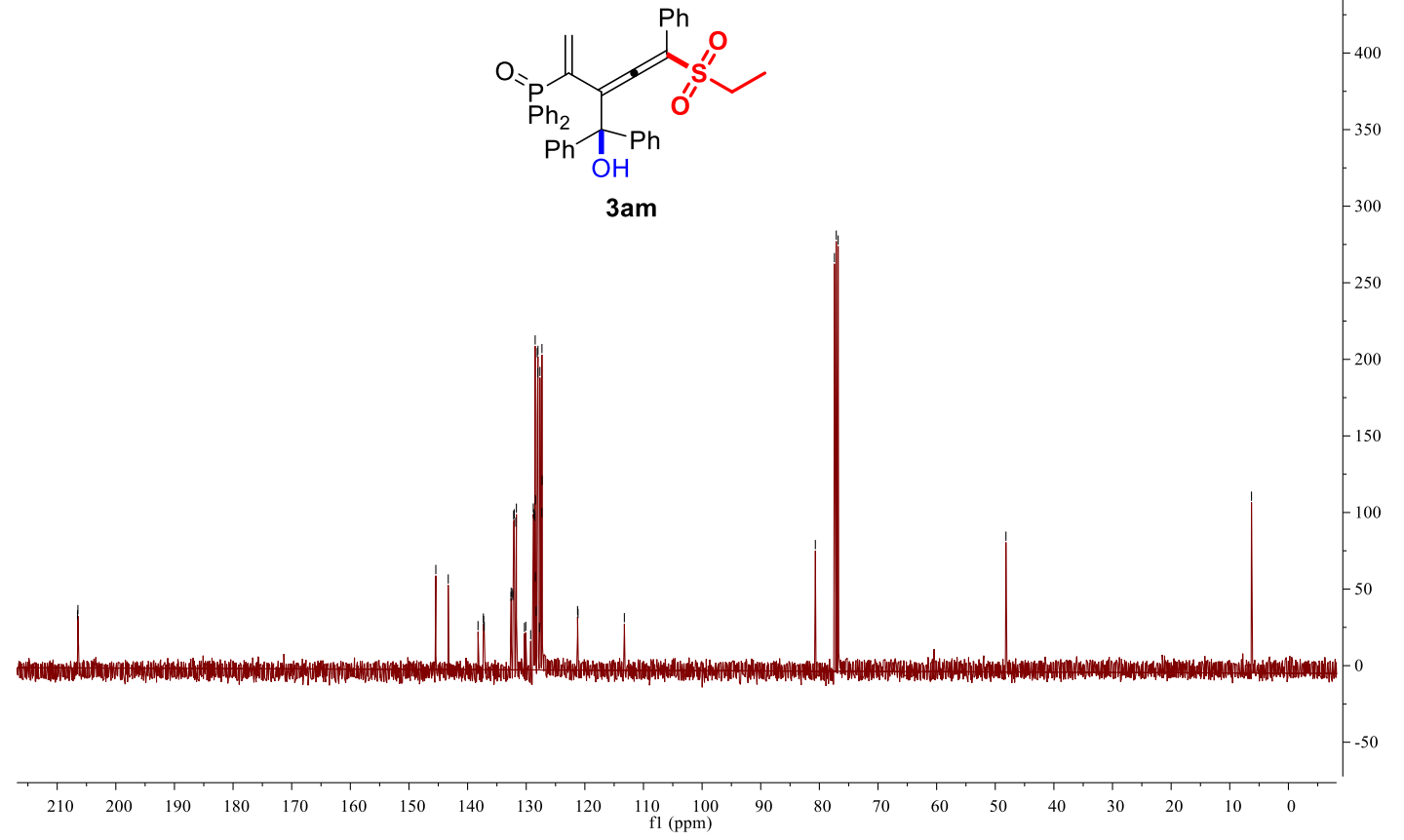

${ }^{13} \mathrm{C}$ NMR $\left(101 \mathrm{MHz}, \mathrm{CDCl}_{3}\right)$ of $\mathbf{3 a m}$

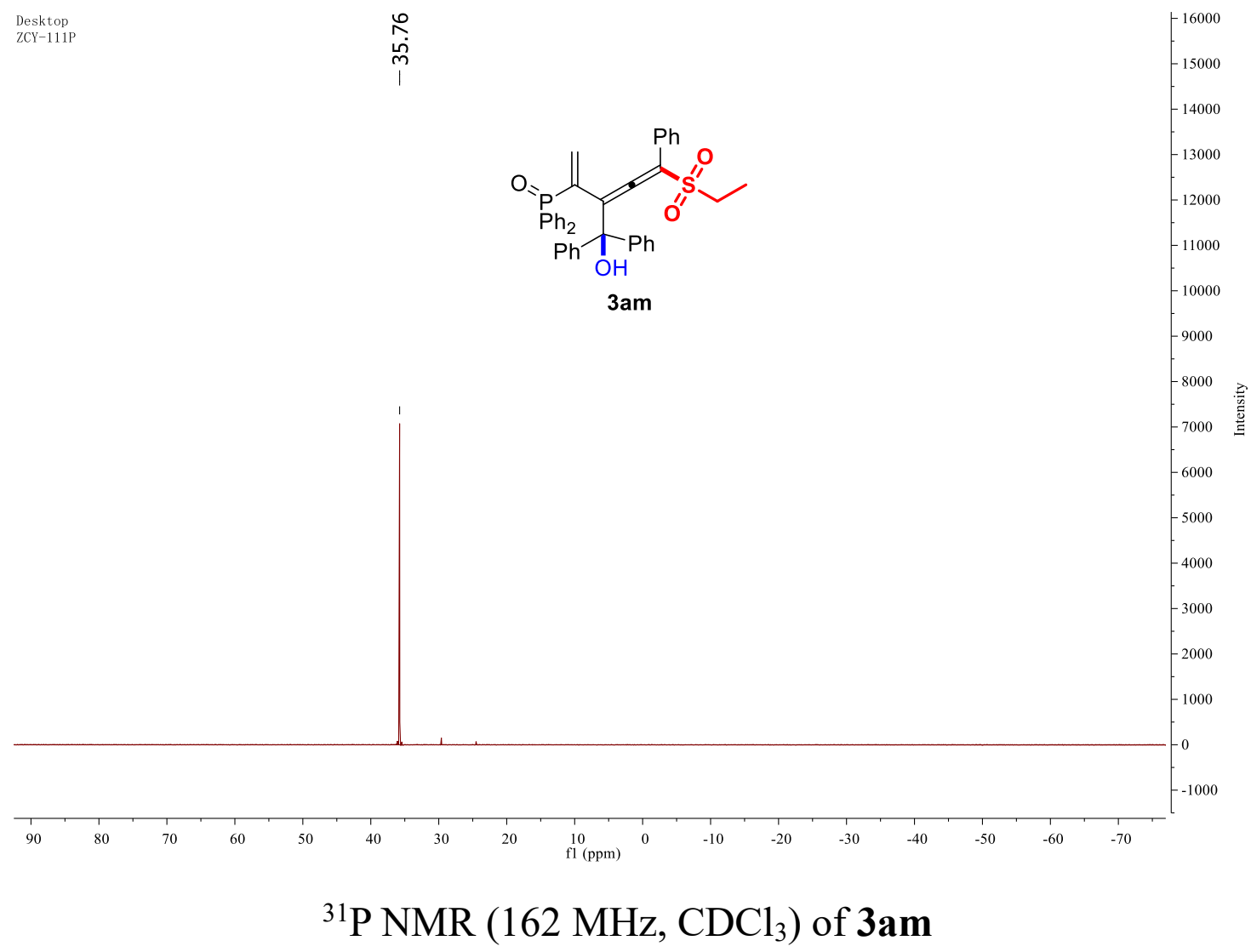


20190524-9 \#9 RT: 0.14 AV: 1 SB: 36 0.01-0.04, 0.47-1.02 NL: $1.94 E 7$ T: FTMS $\{1,1\}+p$ ESI Full ms [100.00-1000.00]

$$
599.18112
$$
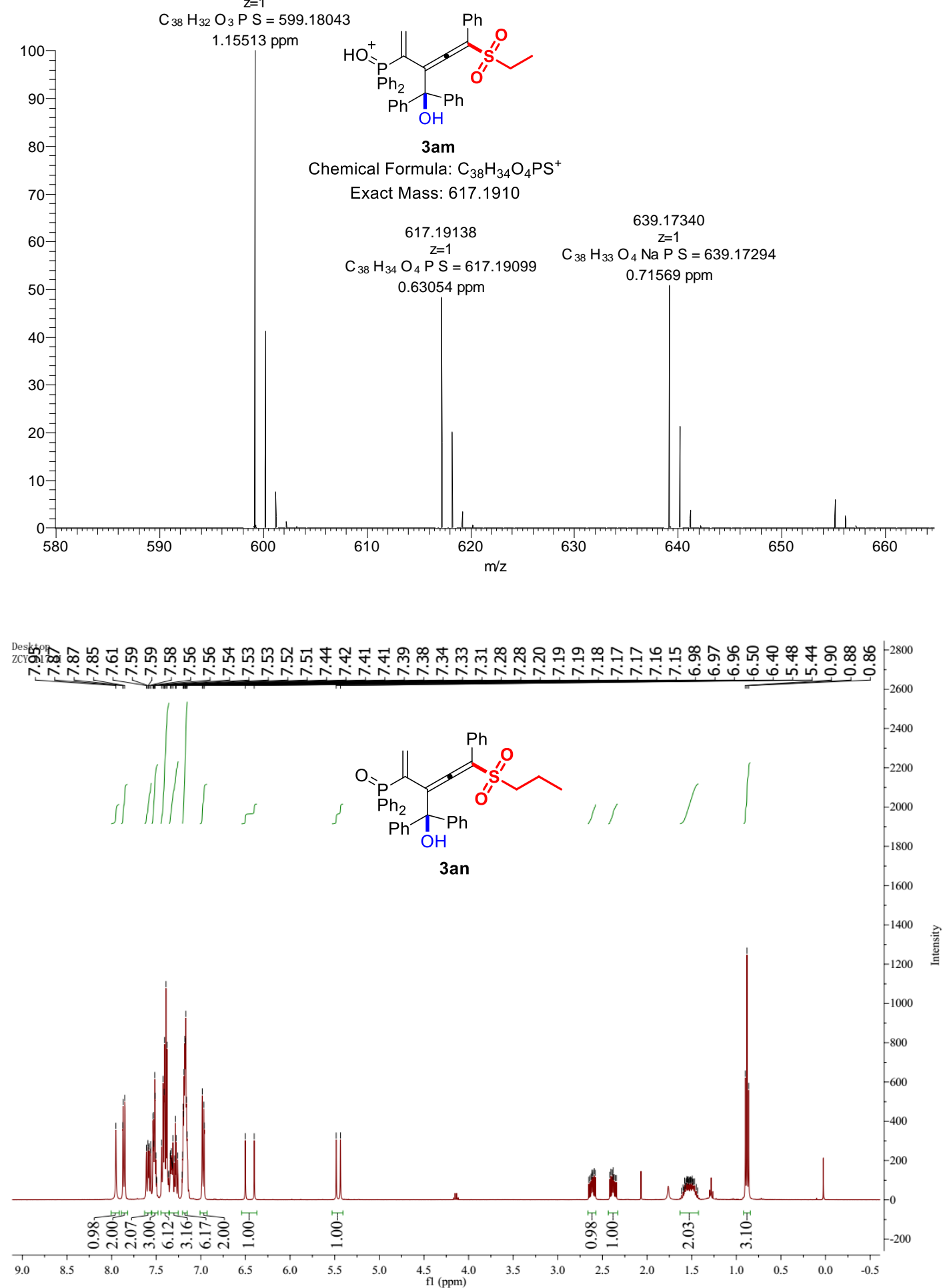

${ }^{1} \mathrm{H}$ NMR $\left(400 \mathrm{MHz}, \mathrm{CDCl}_{3}\right)$ of $\mathbf{3 a n}$ 


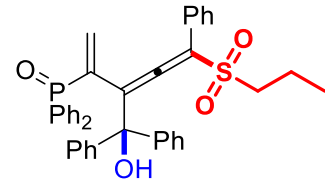

3an

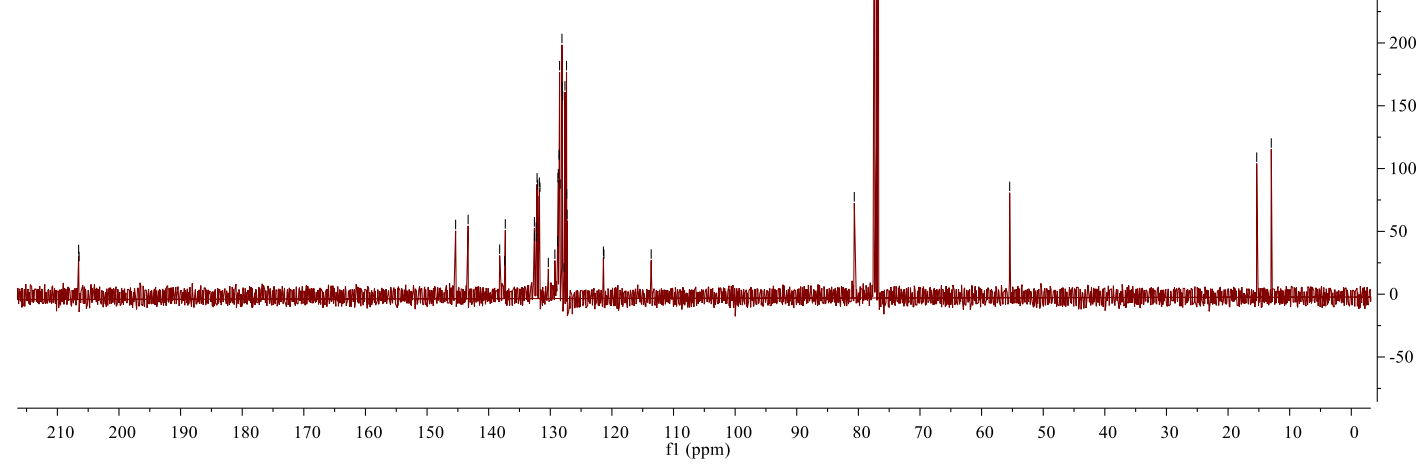

${ }^{13} \mathrm{C}$ NMR $\left(101 \mathrm{MHz}, \mathrm{CDCl}_{3}\right)$ of $\mathbf{3 a n}$

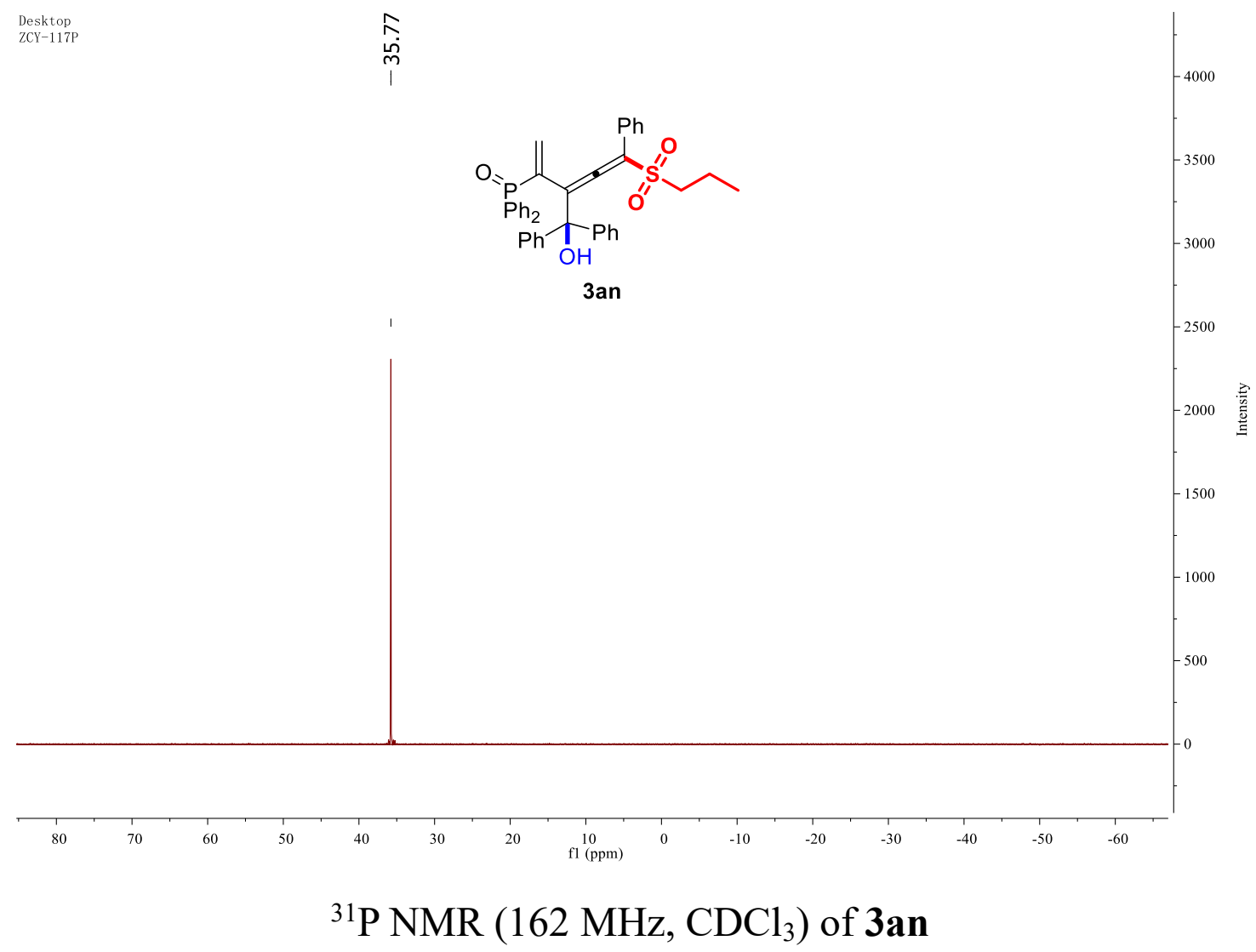



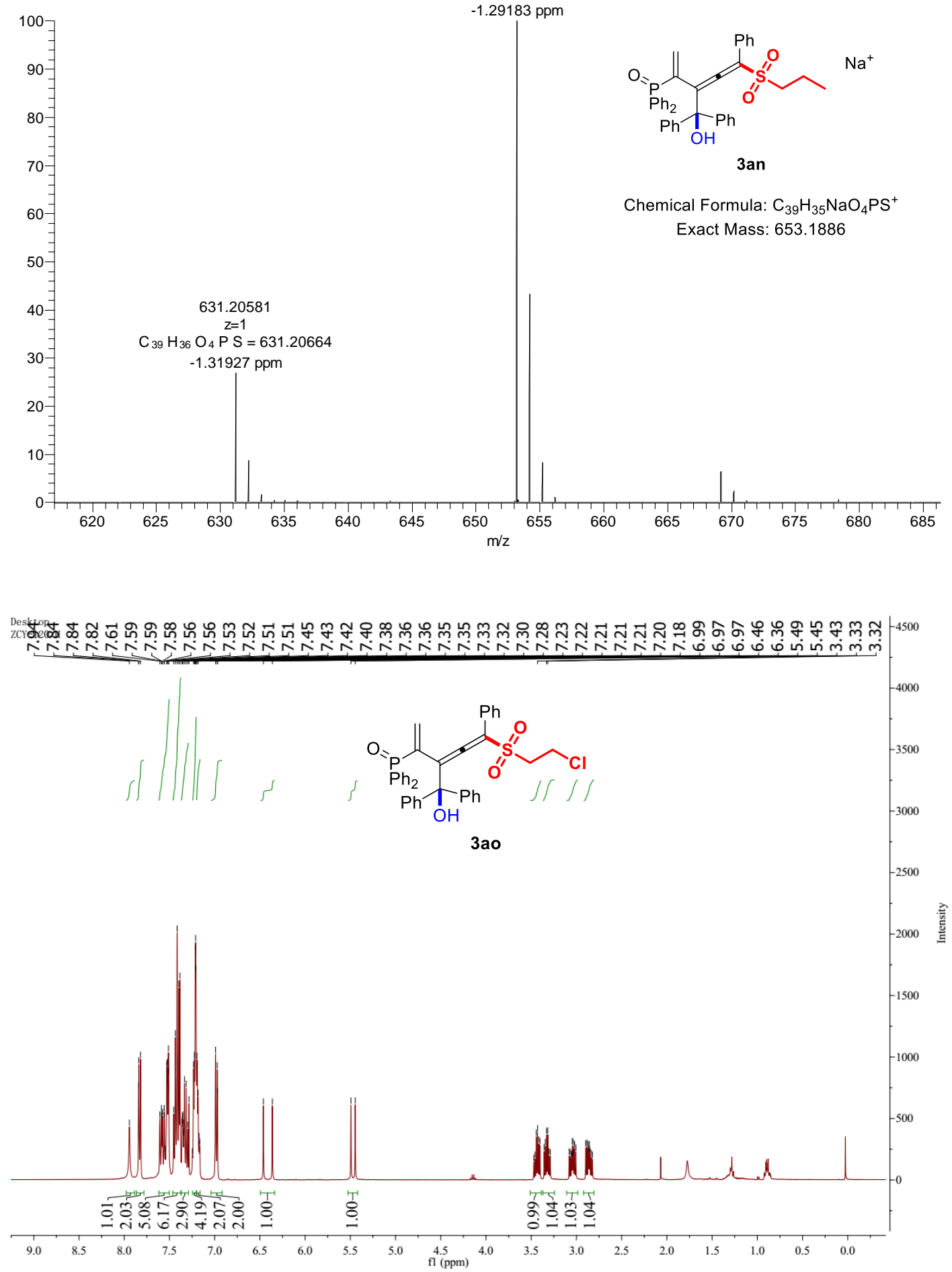

${ }^{1} \mathrm{H}$ NMR (400 MHz, $\left.\mathrm{CDCl}_{3}\right)$ of $\mathbf{3 a o}$ 


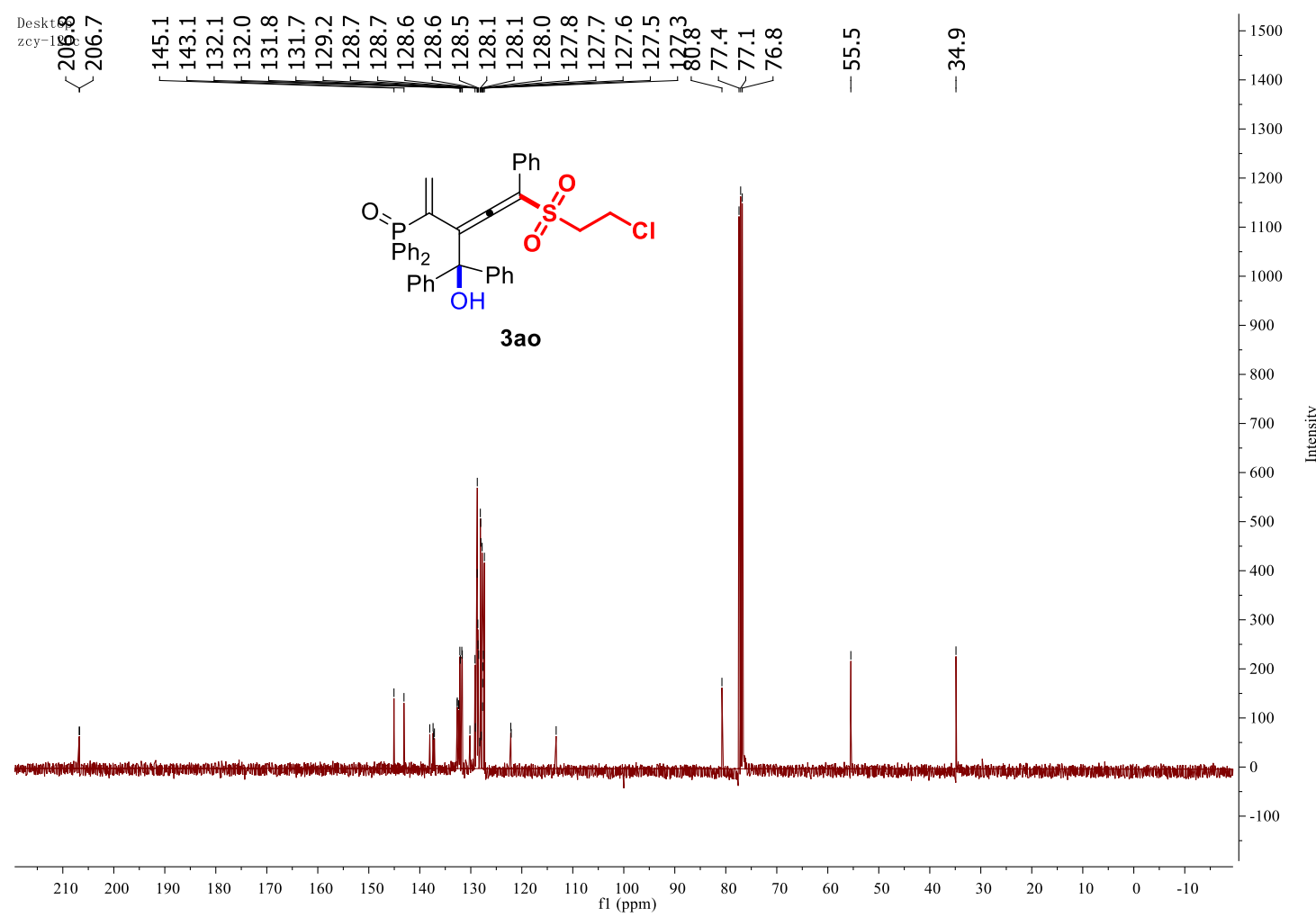

\section{${ }^{13} \mathrm{C}$ NMR $\left(101 \mathrm{MHz}, \mathrm{CDCl}_{3}\right)$ of $\mathbf{3 a o}$}

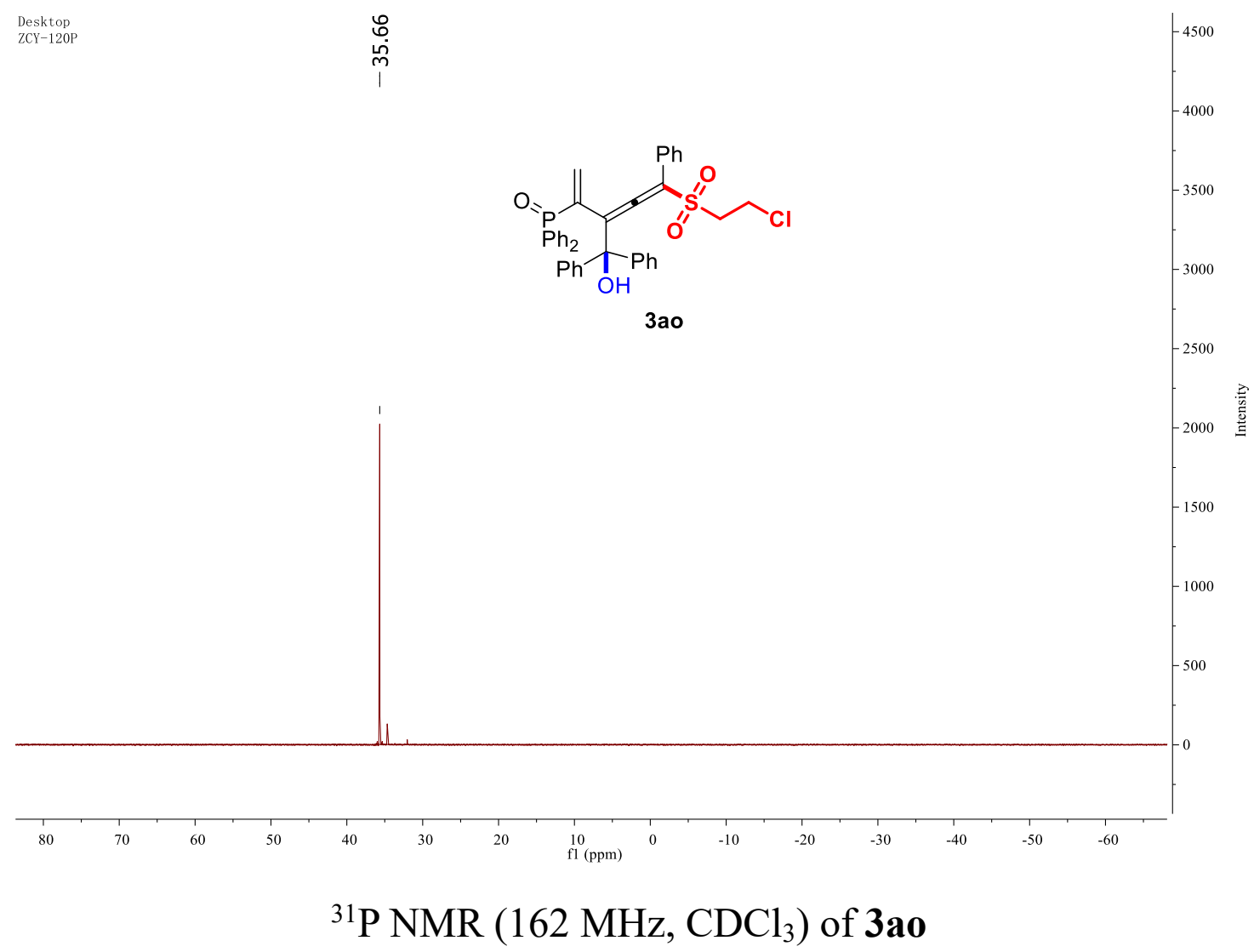



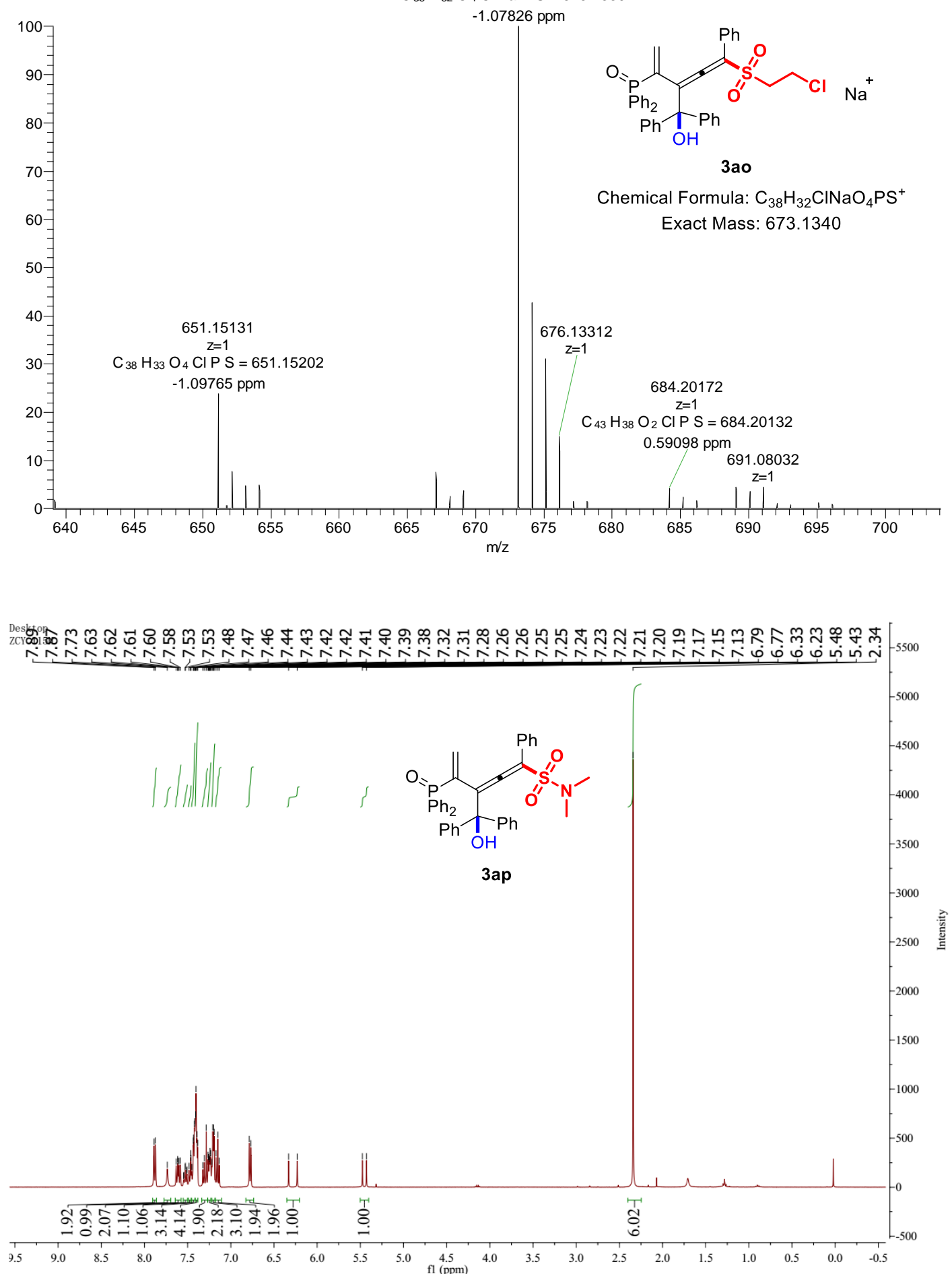

${ }^{1} \mathrm{H}$ NMR (400 MHz, $\mathrm{CDCl}_{3}$ ) of 3ap 


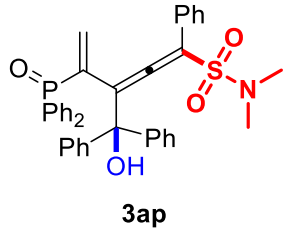

$\hat{i}$

${ }^{13} \mathrm{C}$ NMR $\left(101 \mathrm{MHz}, \mathrm{CDCl}_{3}\right)$ of 3ap

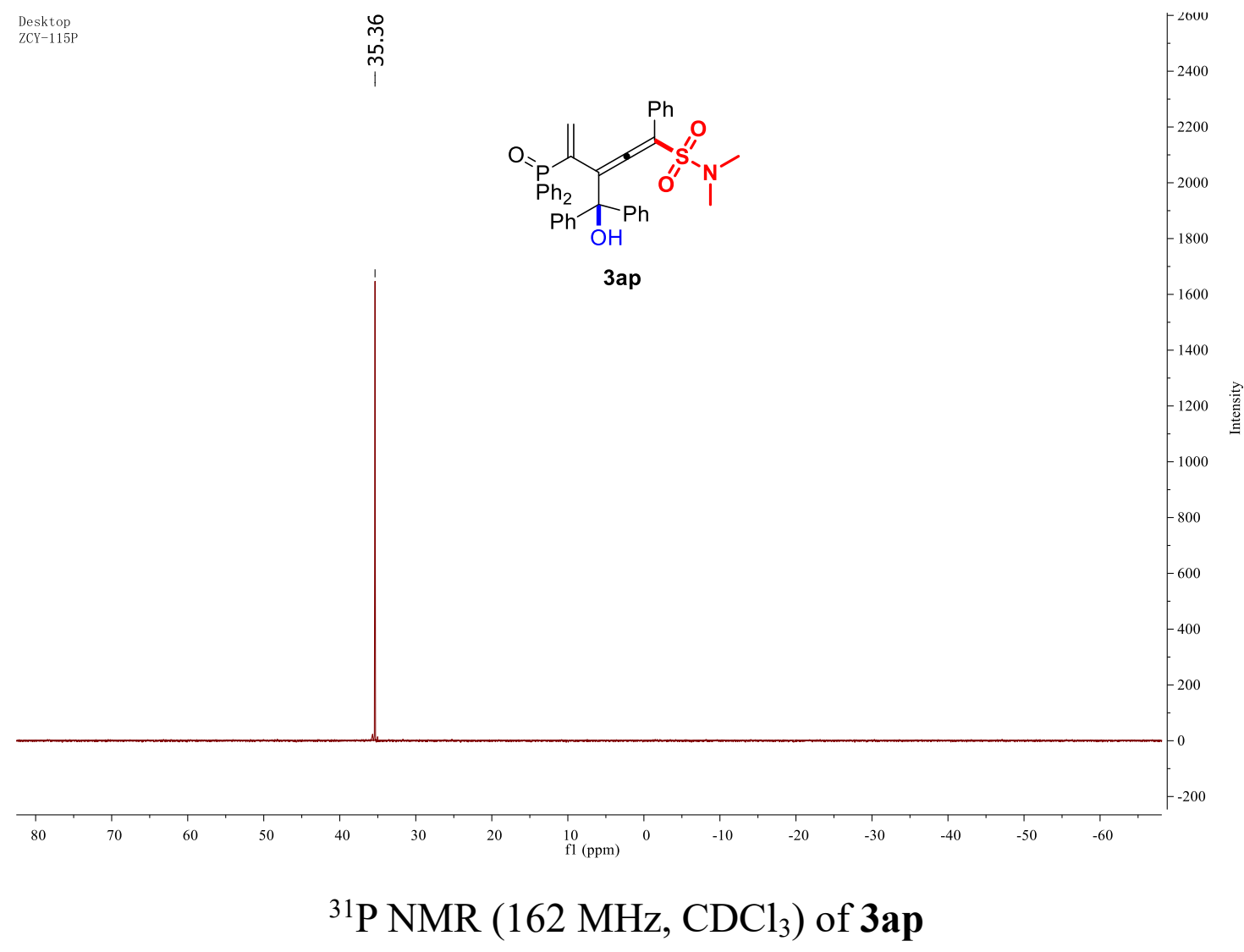



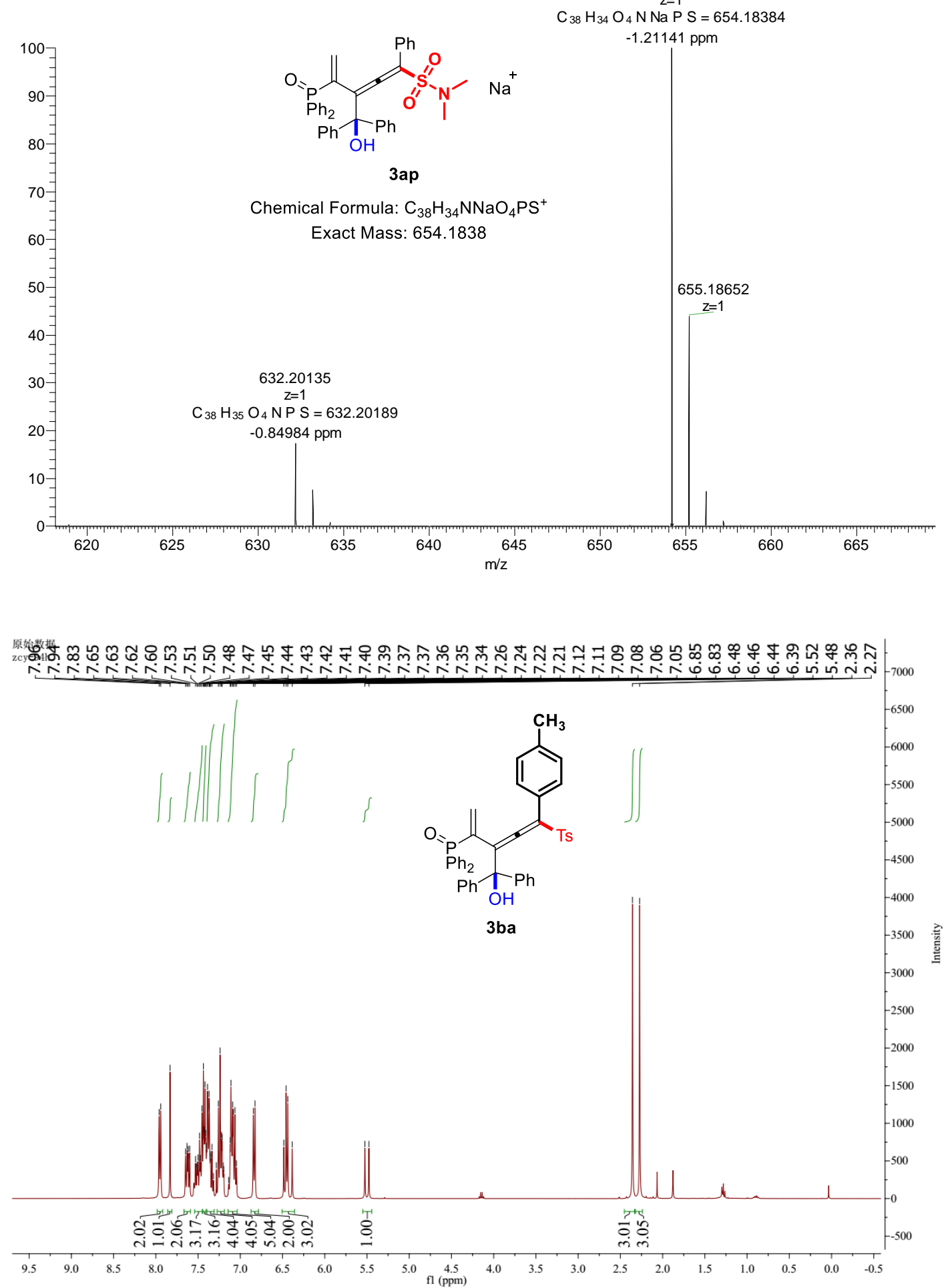

${ }^{1} \mathrm{H}$ NMR $\left(400 \mathrm{MHz}, \mathrm{CDCl}_{3}\right)$ of $\mathbf{3 b a}$ 


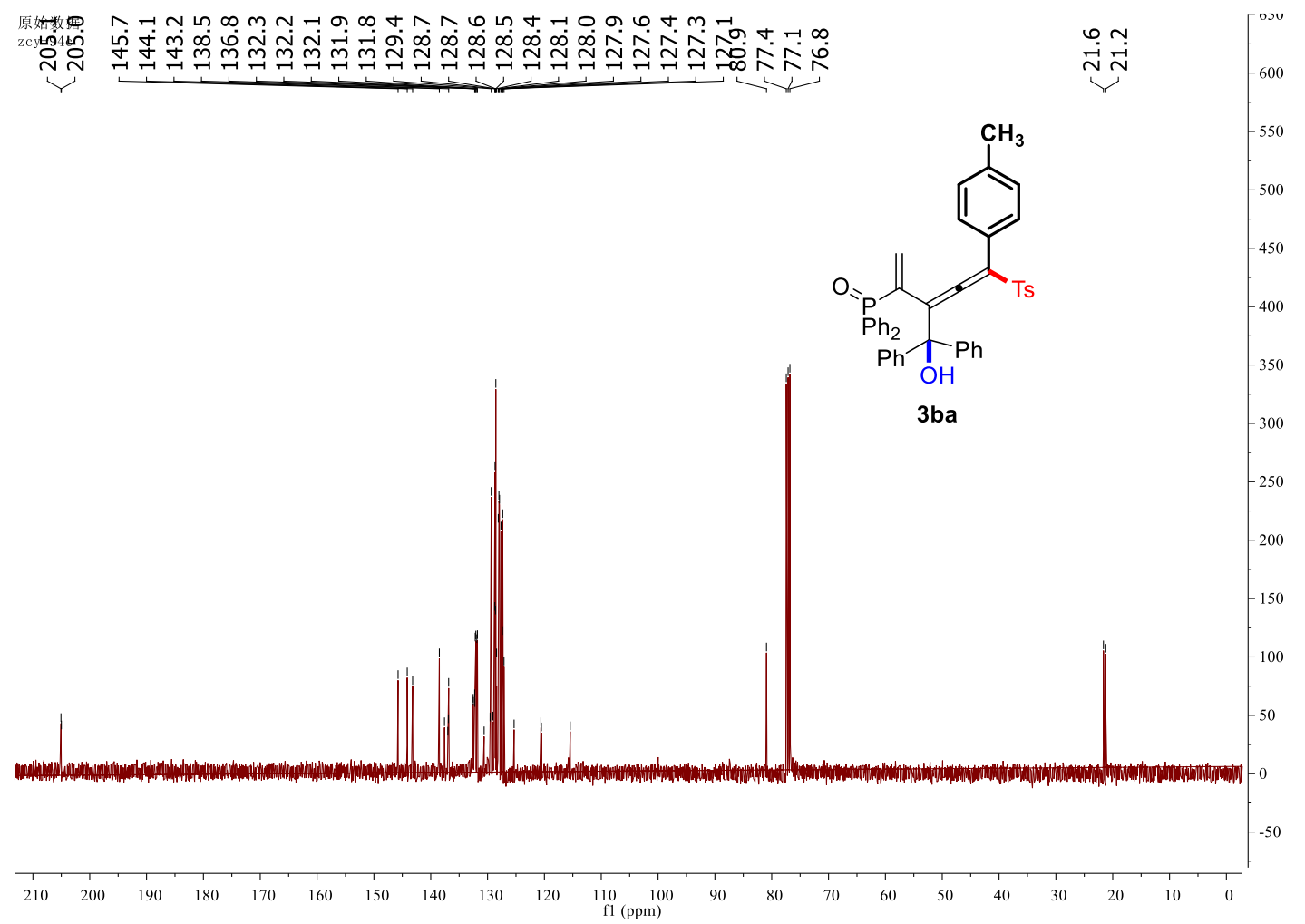

\section{${ }^{13} \mathrm{C}$ NMR $\left(101 \mathrm{MHz}, \mathrm{CDCl}_{3}\right)$ of $\mathbf{3 b a}$}

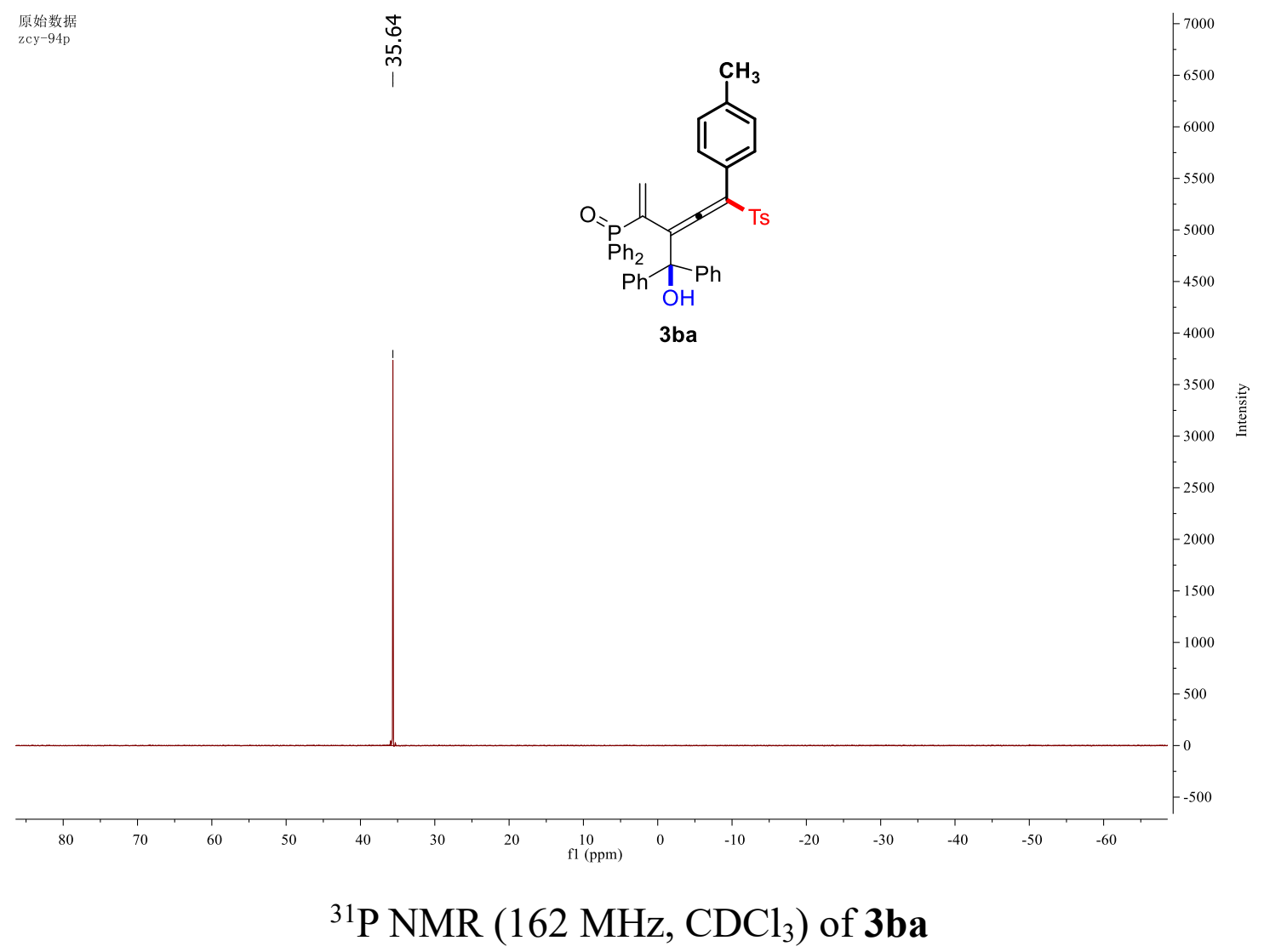


20190506-24 \#31 RT: 0.48 AV: 1 SB: 20 0.01-0.07, 0.78-1.02 NL: $1.53 E 5$ T: FTMS $\{1,1\}+p$ ESI Full ms [100.00-1000.00] 675.21089 $\mathrm{C}_{44} \mathrm{H}_{36} \mathrm{O}_{3} \mathrm{P} \mathrm{S}=675.21173$
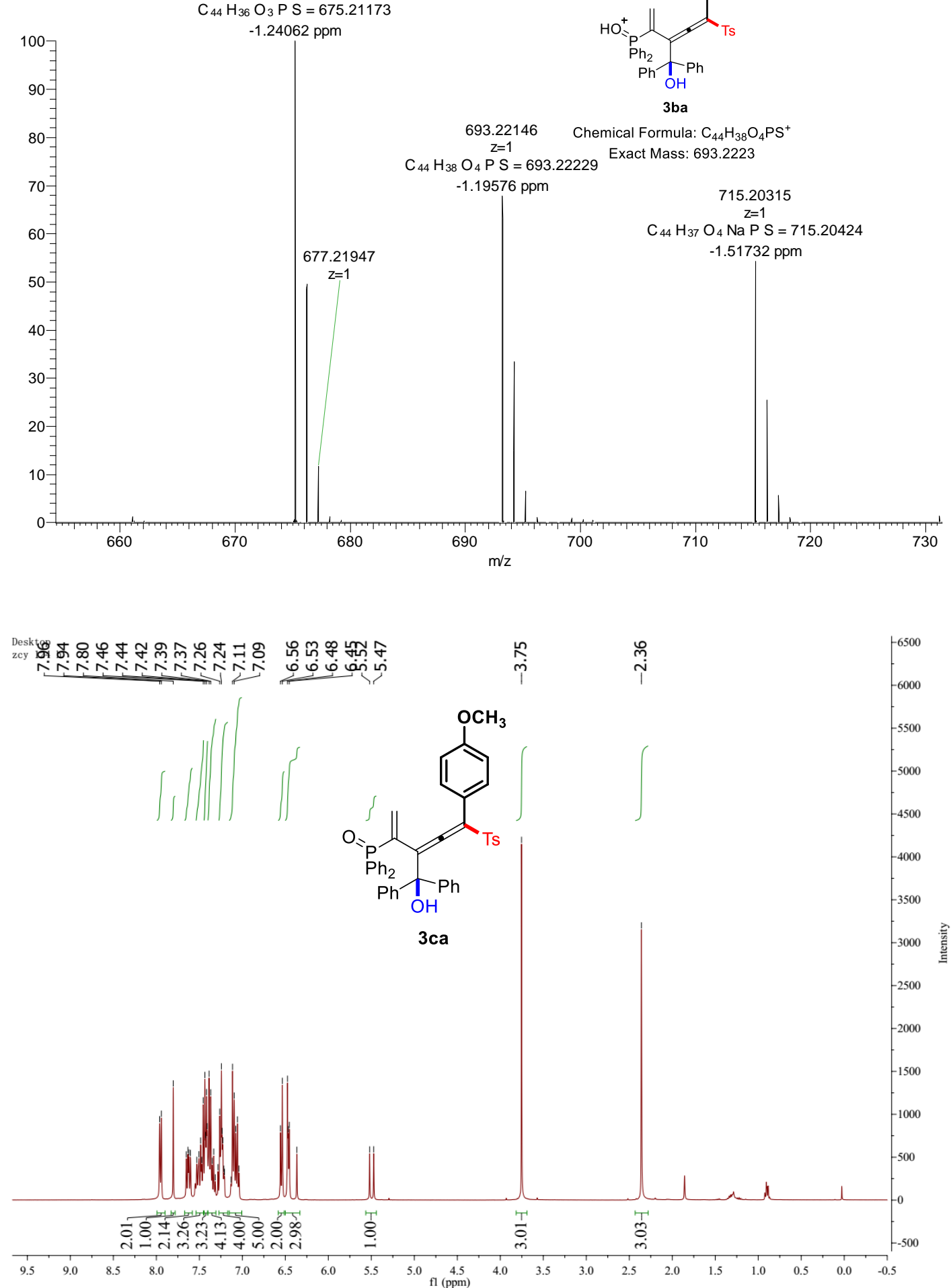

${ }^{1} \mathrm{H}$ NMR $\left(400 \mathrm{MHz}, \mathrm{CDCl}_{3}\right)$ of $\mathbf{3 c a}$ 


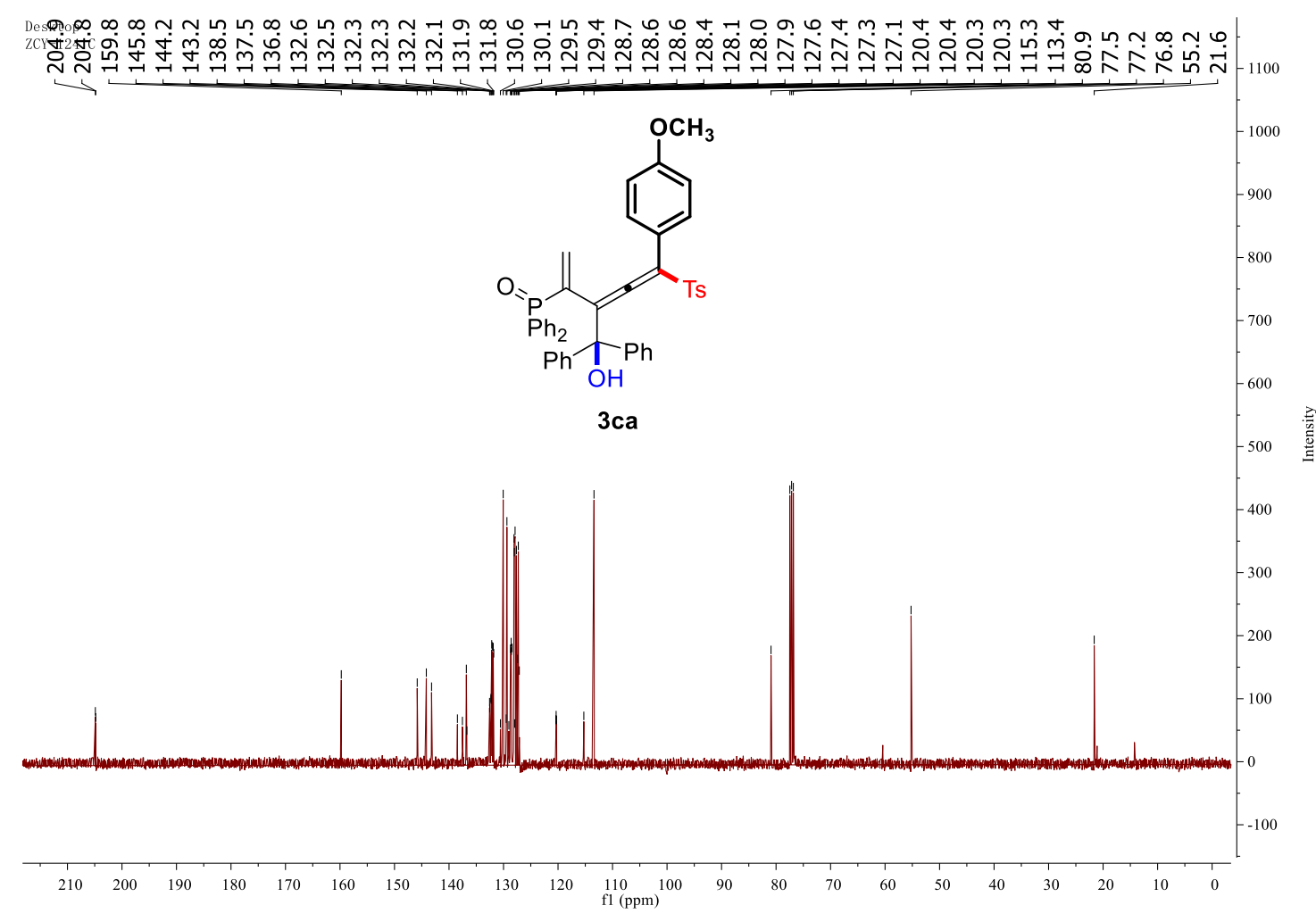

\section{${ }^{13} \mathrm{C}$ NMR $\left(101 \mathrm{MHz}, \mathrm{CDCl}_{3}\right)$ of $\mathbf{3 c a}$}

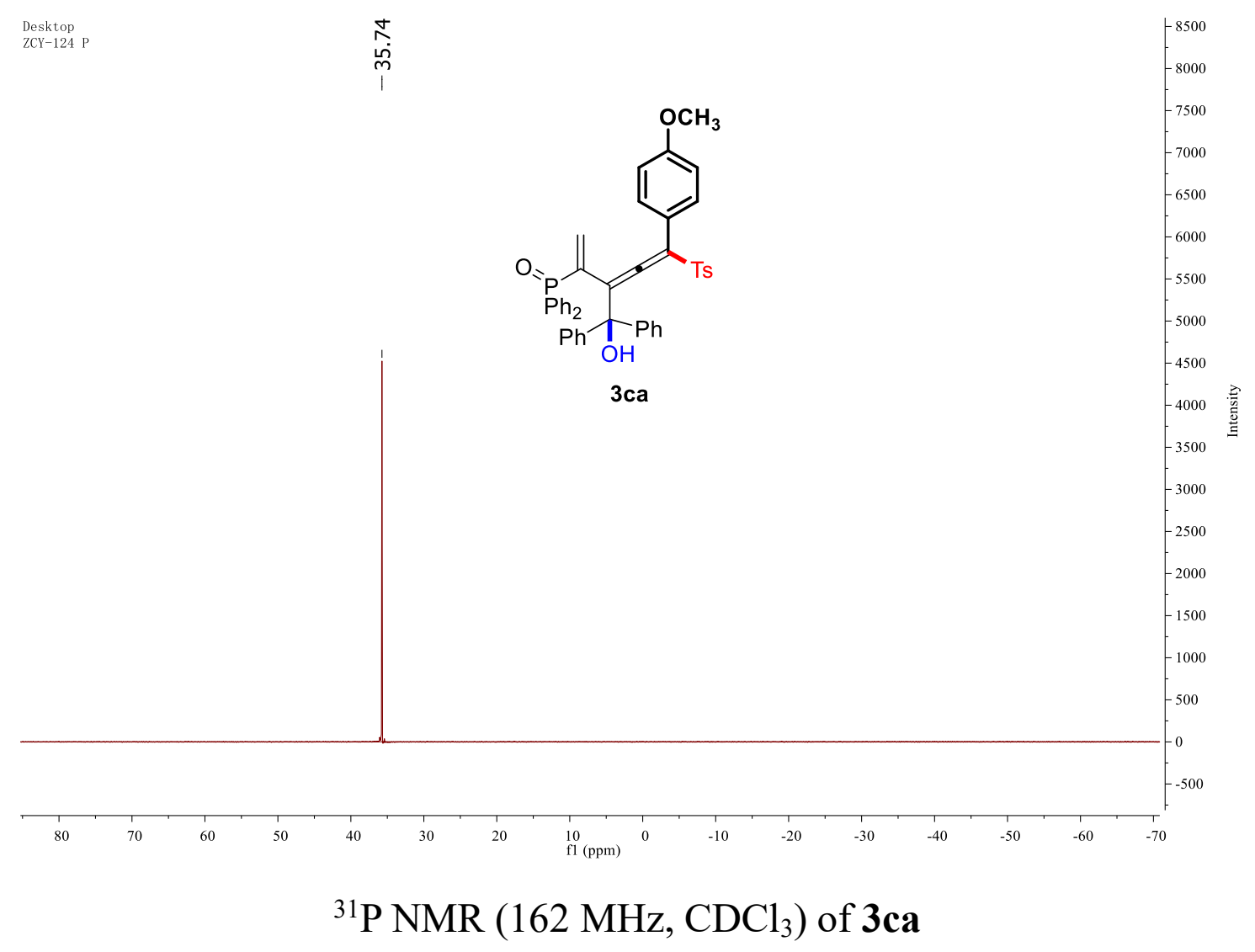


731.19812 $\mathrm{z}=1$ $\mathrm{C}_{44} \mathrm{H}_{37} \mathrm{O}_{5} \mathrm{Na} \mathrm{P} \mathrm{S}=731.19915$

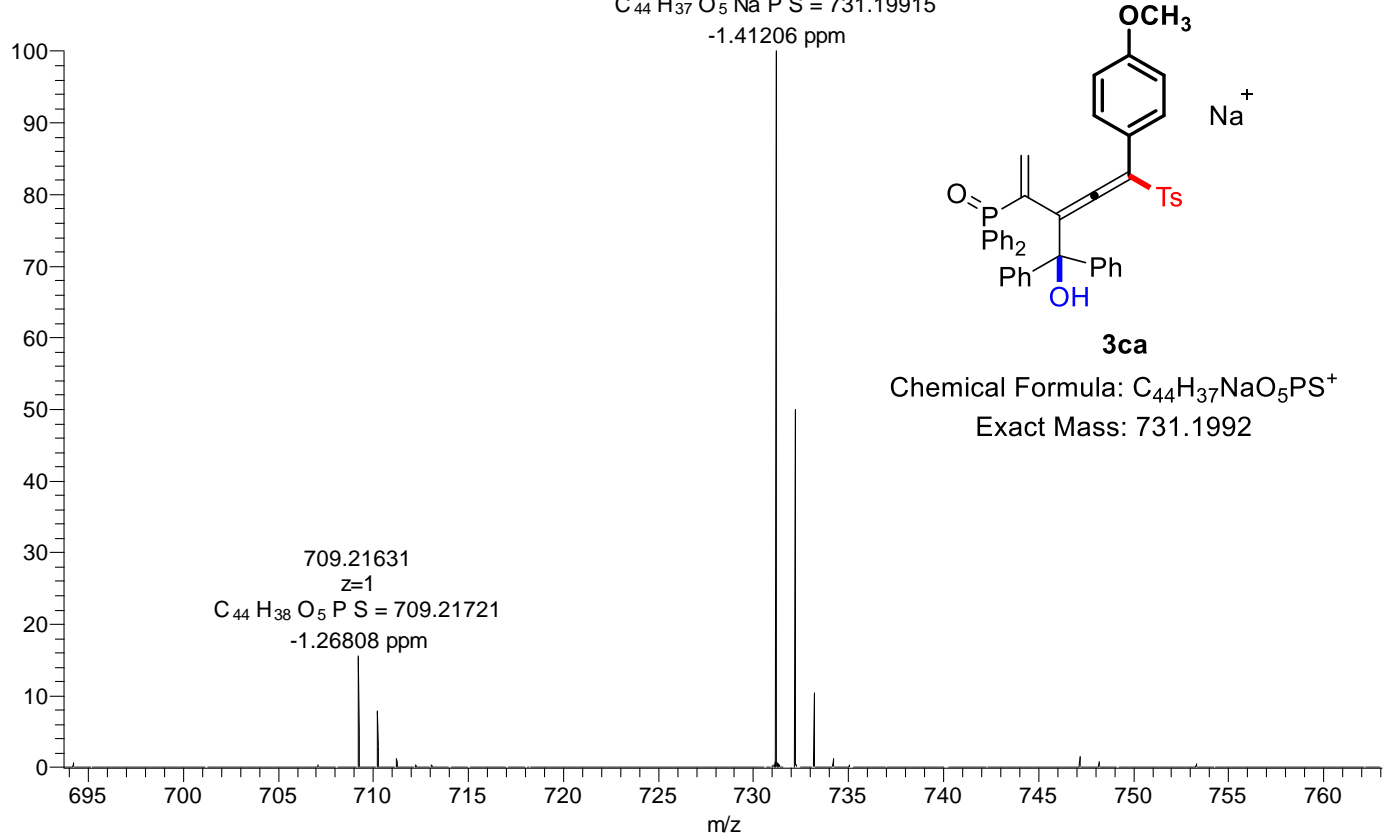

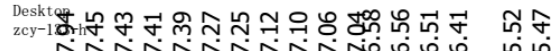

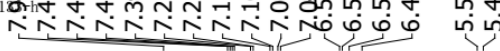

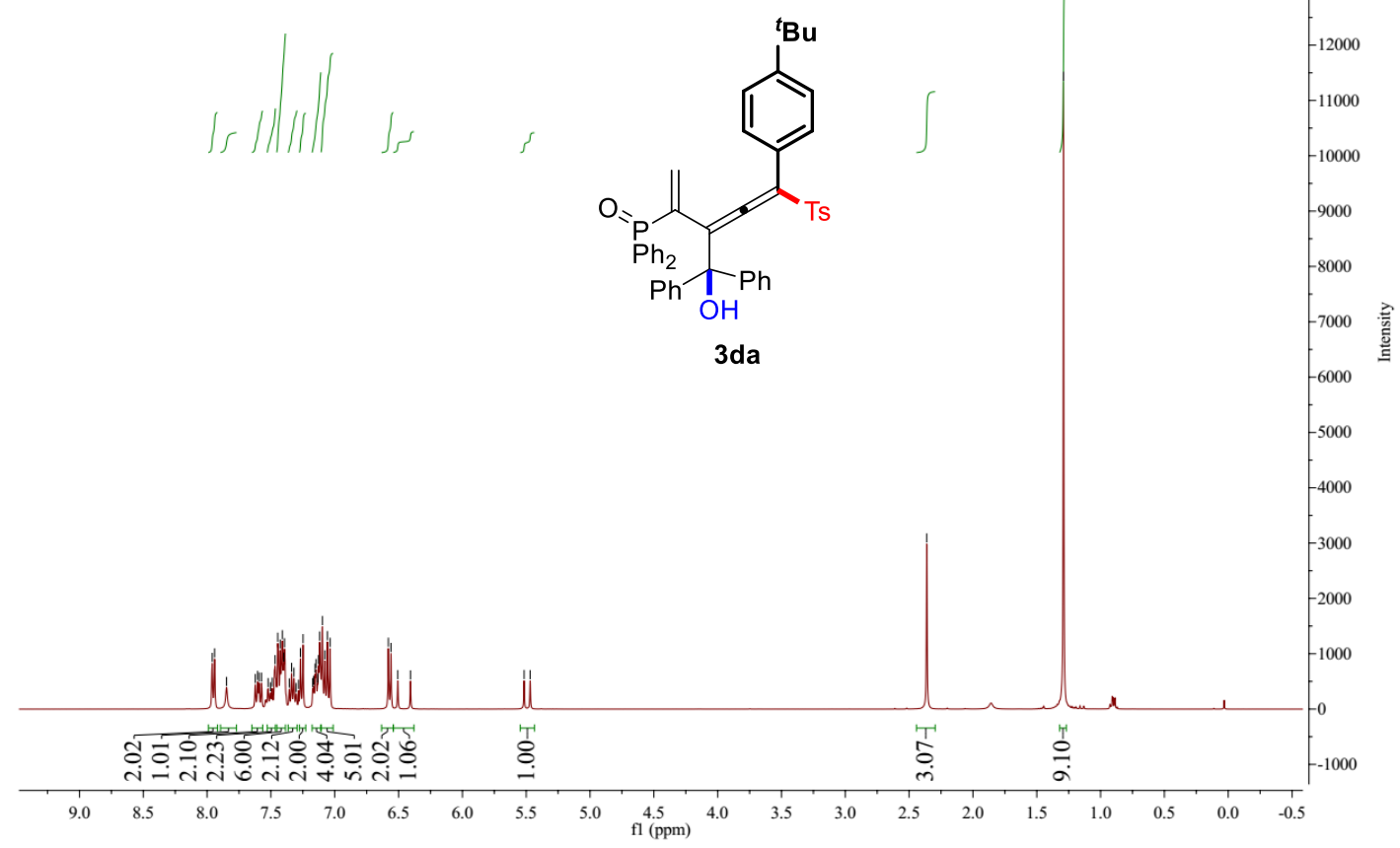

${ }^{1} \mathrm{H}$ NMR $\left(400 \mathrm{MHz}, \mathrm{CDCl}_{3}\right)$ of $\mathbf{3 d a}$ 


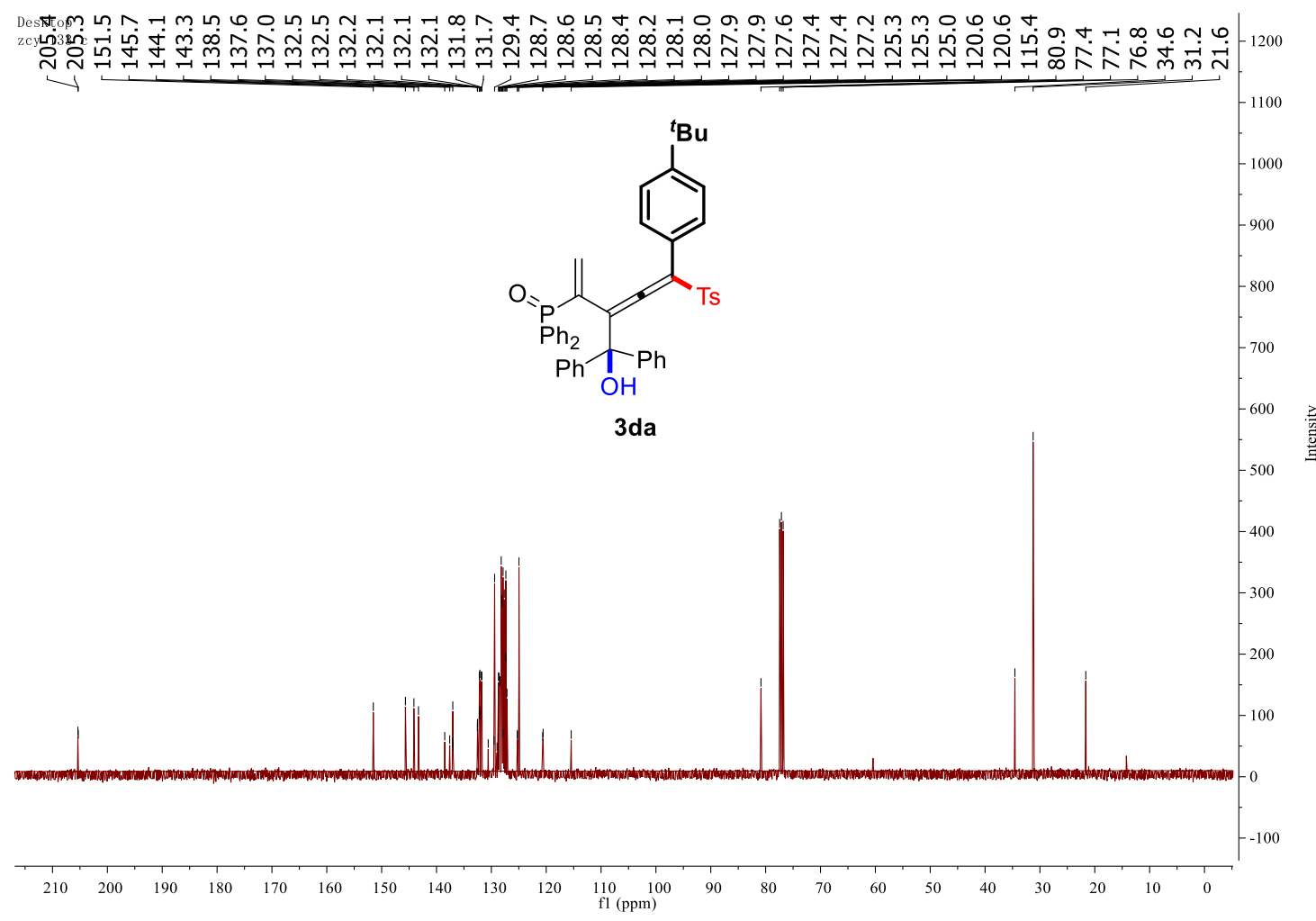

\section{${ }^{13} \mathrm{C}$ NMR $\left(101 \mathrm{MHz}, \mathrm{CDCl}_{3}\right)$ of $\mathbf{3 d a}$}

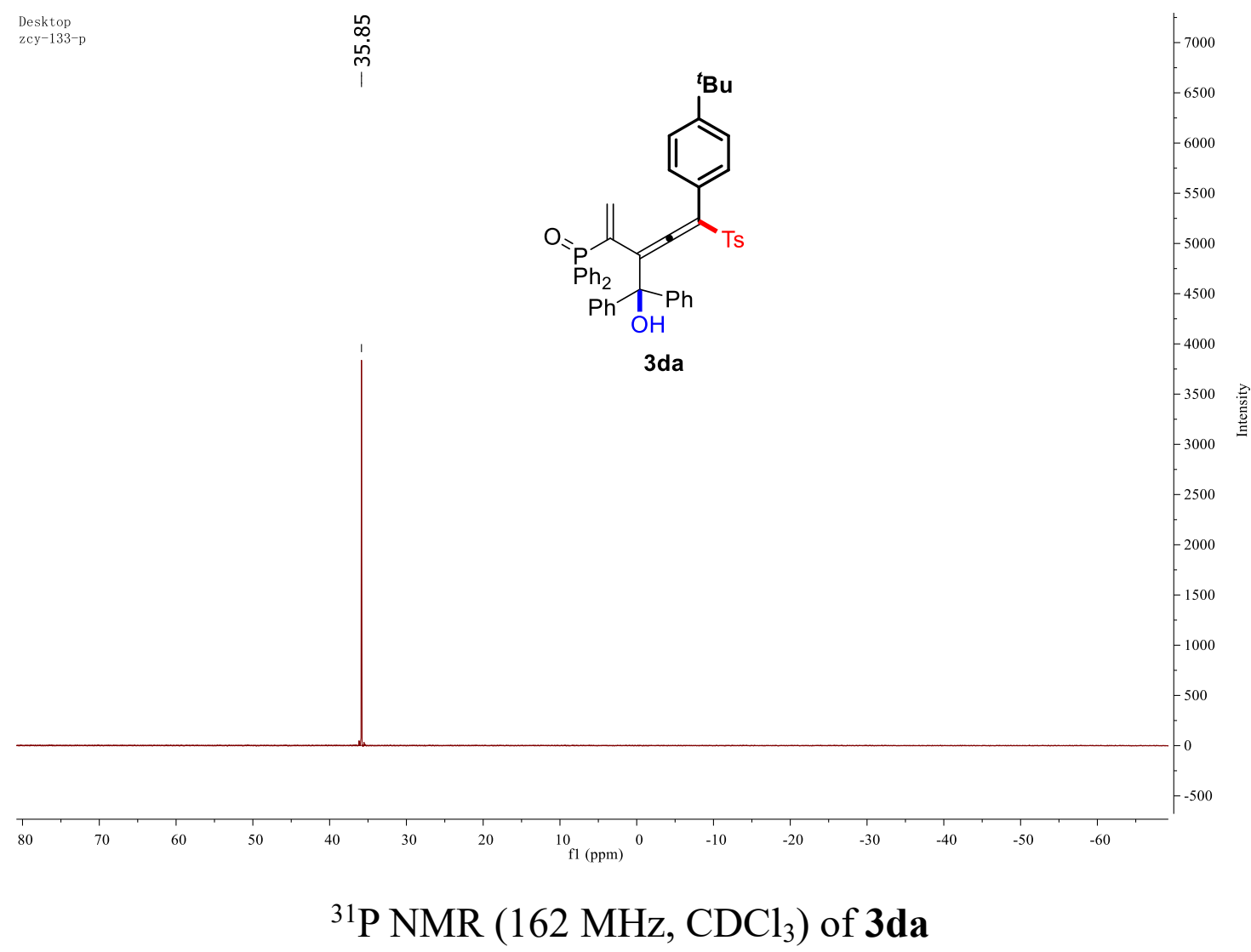


16 \#27 RT: 0.41 AV: 1 NL: 1.60E5

T: FTMS $\{1,1\}+$ p ESI Full ms [100.00-1000.00]

757.25037

$\mathrm{z}=1$

$\mathrm{C}_{47} \mathrm{H}_{43} \mathrm{O}_{4} \mathrm{Na} \mathrm{P} \mathrm{S}=757.25119$
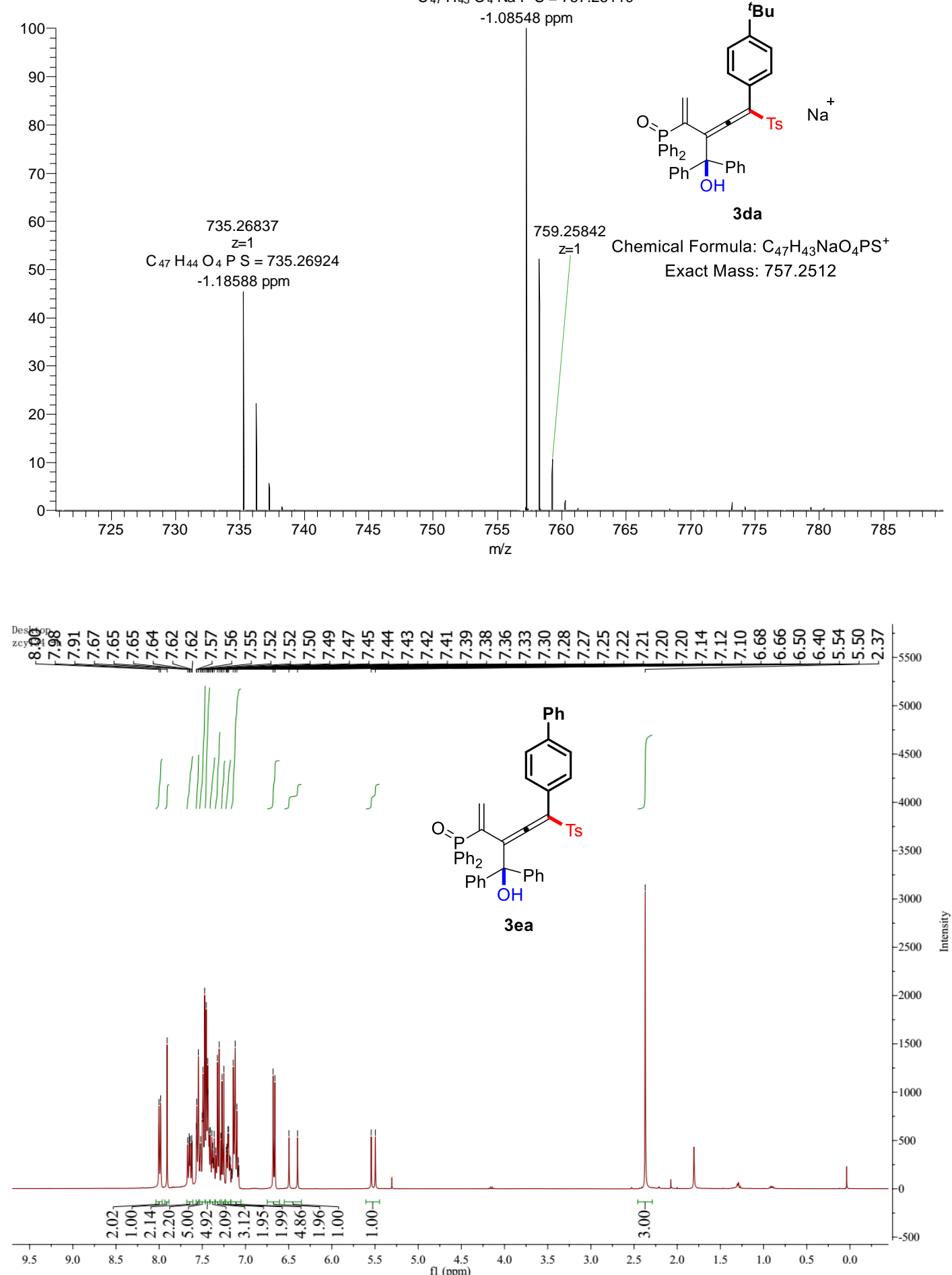

${ }^{1} \mathrm{H}$ NMR $\left(400 \mathrm{MHz}, \mathrm{CDCl}_{3}\right)$ of 3ea 


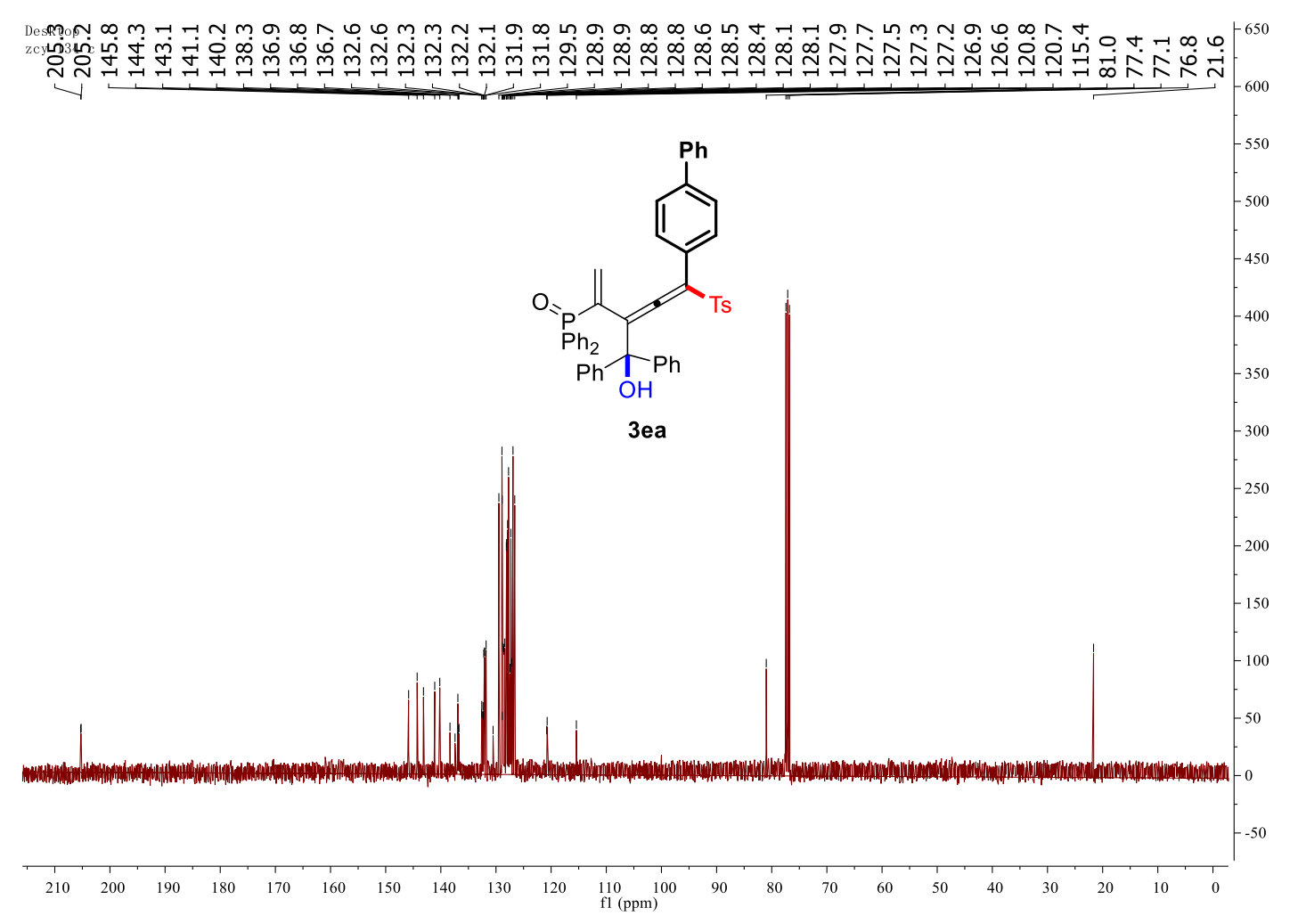

\section{${ }^{13} \mathrm{C}$ NMR $\left(101 \mathrm{MHz}, \mathrm{CDCl}_{3}\right)$ of $\mathbf{3 e a}$}

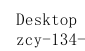

م.

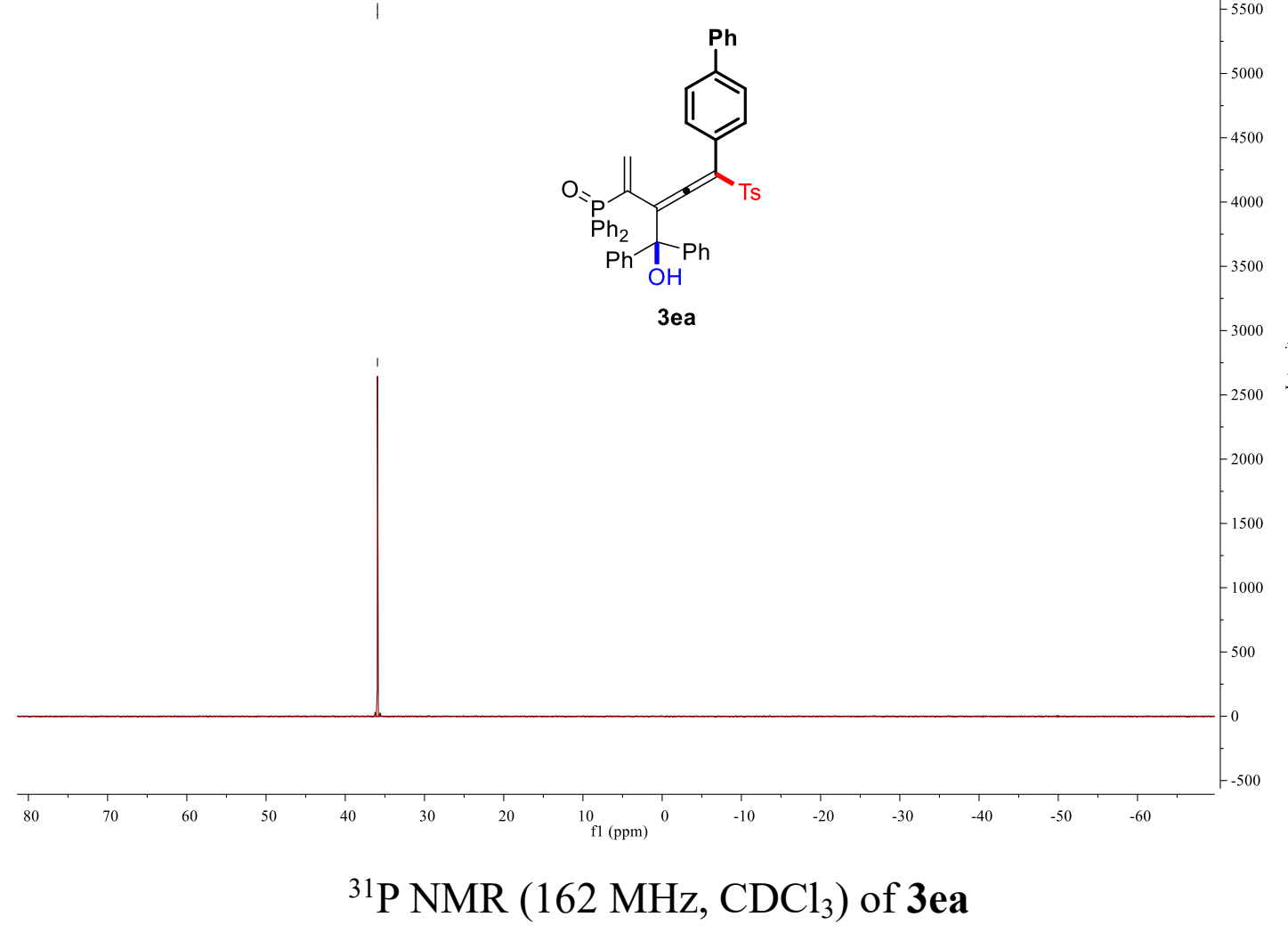


17 \#29 RT: $0.44 \quad$ AV: 1 NL: $2.89 E 4$

T: FTMS $\{1,1\}+p$ ESI Full ms [100.00-1000.00]
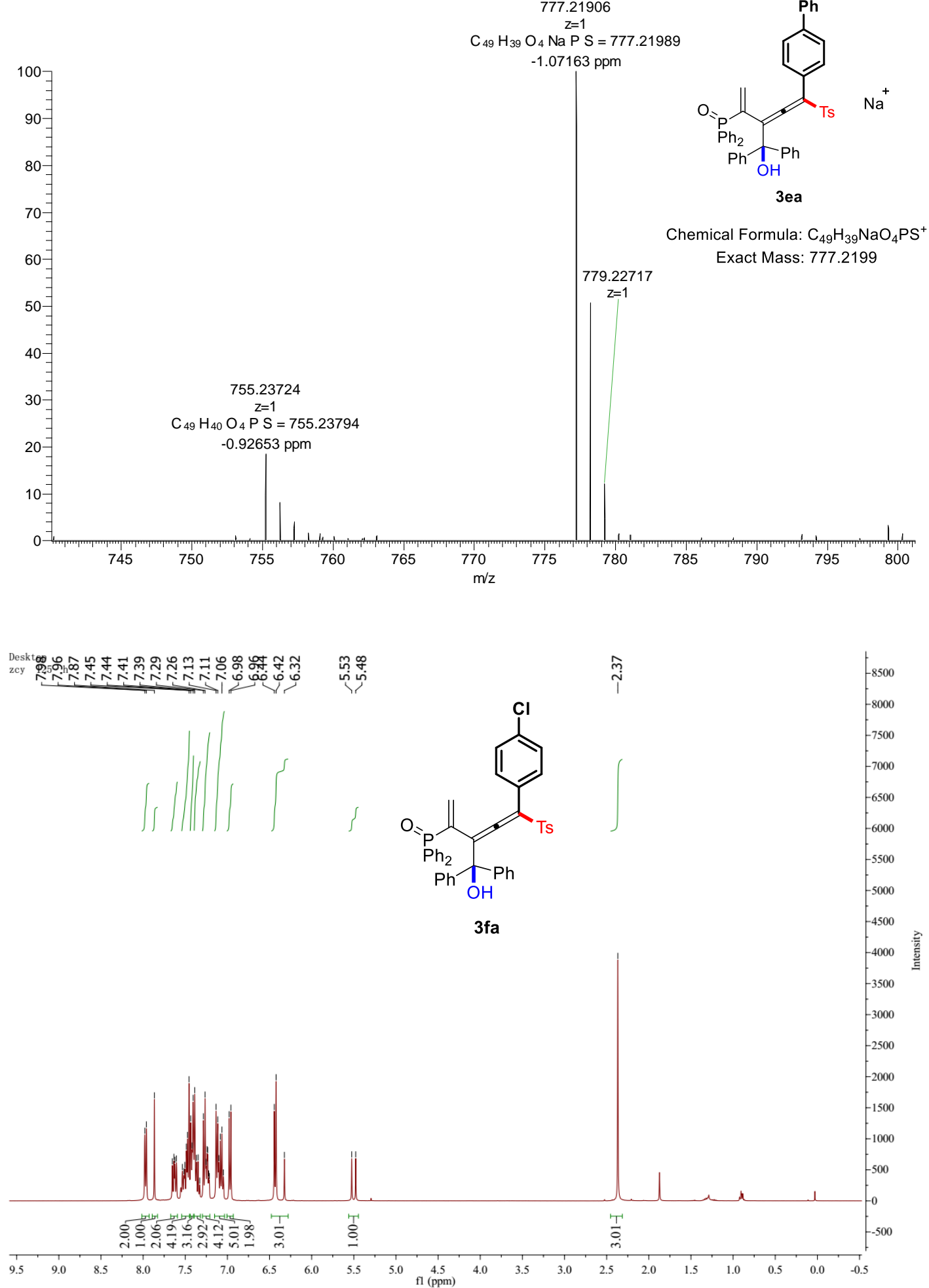

${ }^{1} \mathrm{H}$ NMR $\left(400 \mathrm{MHz}, \mathrm{CDCl}_{3}\right)$ of $\mathbf{3 f a}$ 


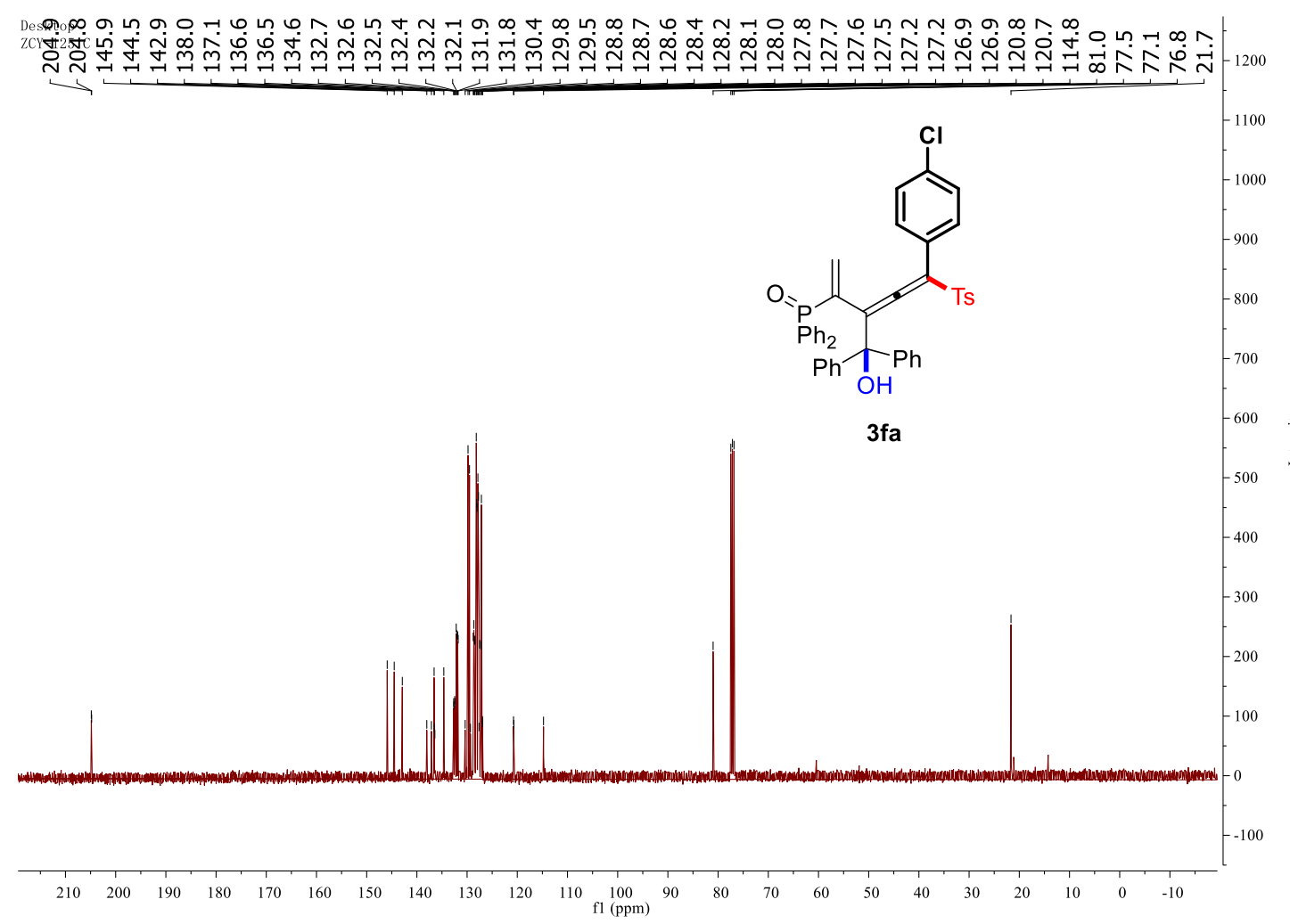

\section{${ }^{13} \mathrm{C} \mathrm{NMR}\left(101 \mathrm{MHz}, \mathrm{CDCl}_{3}\right)$ of $\mathbf{3 f a}$}

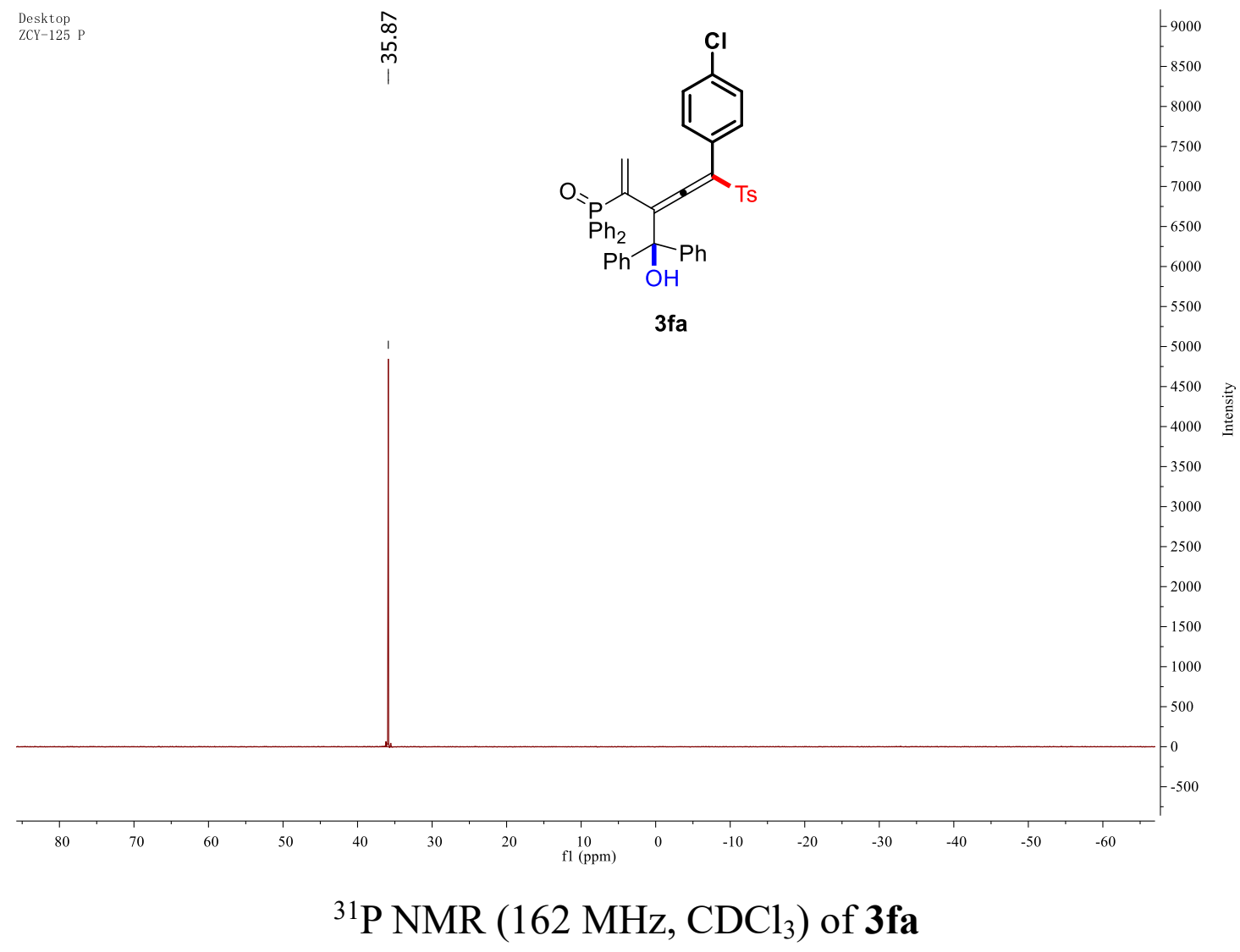


10 \#27 RT: 0.40 AV: 1 NL: $1.25 E 4$

T: FTMS $\{1,1\}+p$ ESI Full ms $[100.00-1000.00]$

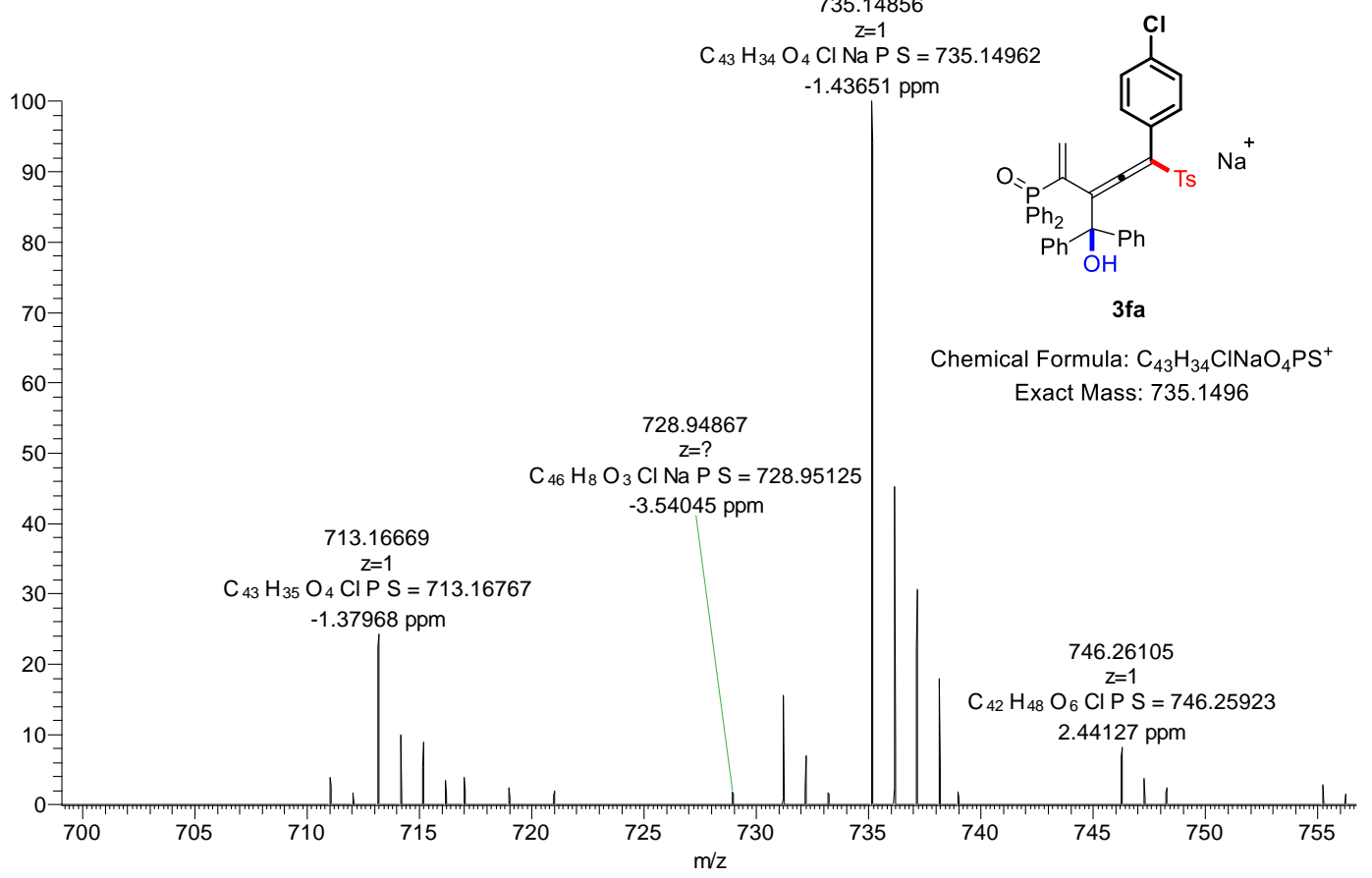

Desktiog

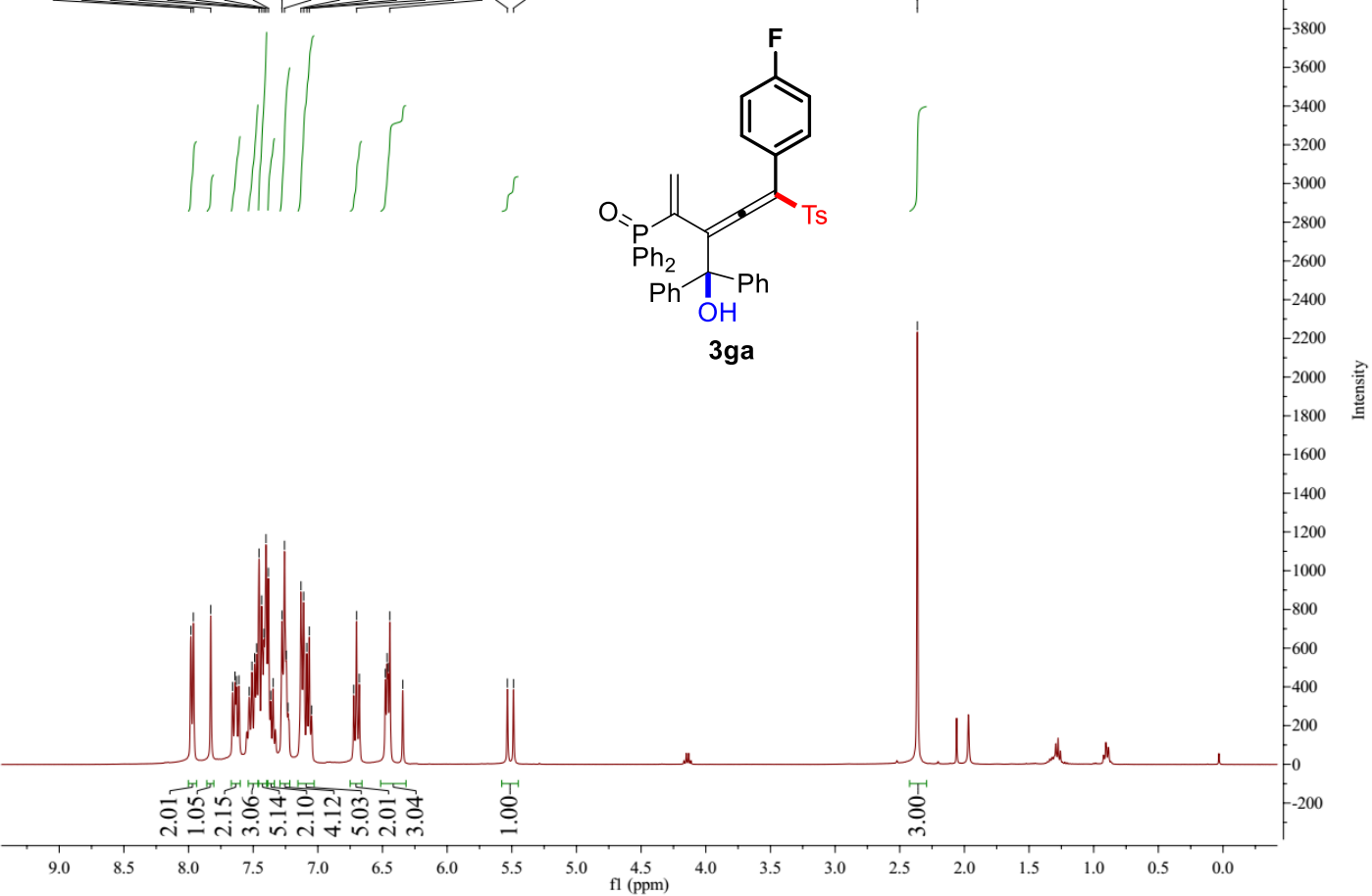

${ }^{1} \mathrm{H}$ NMR (400 MHz, $\left.\mathrm{CDCl}_{3}\right)$ of $\mathbf{3 g a}$ 


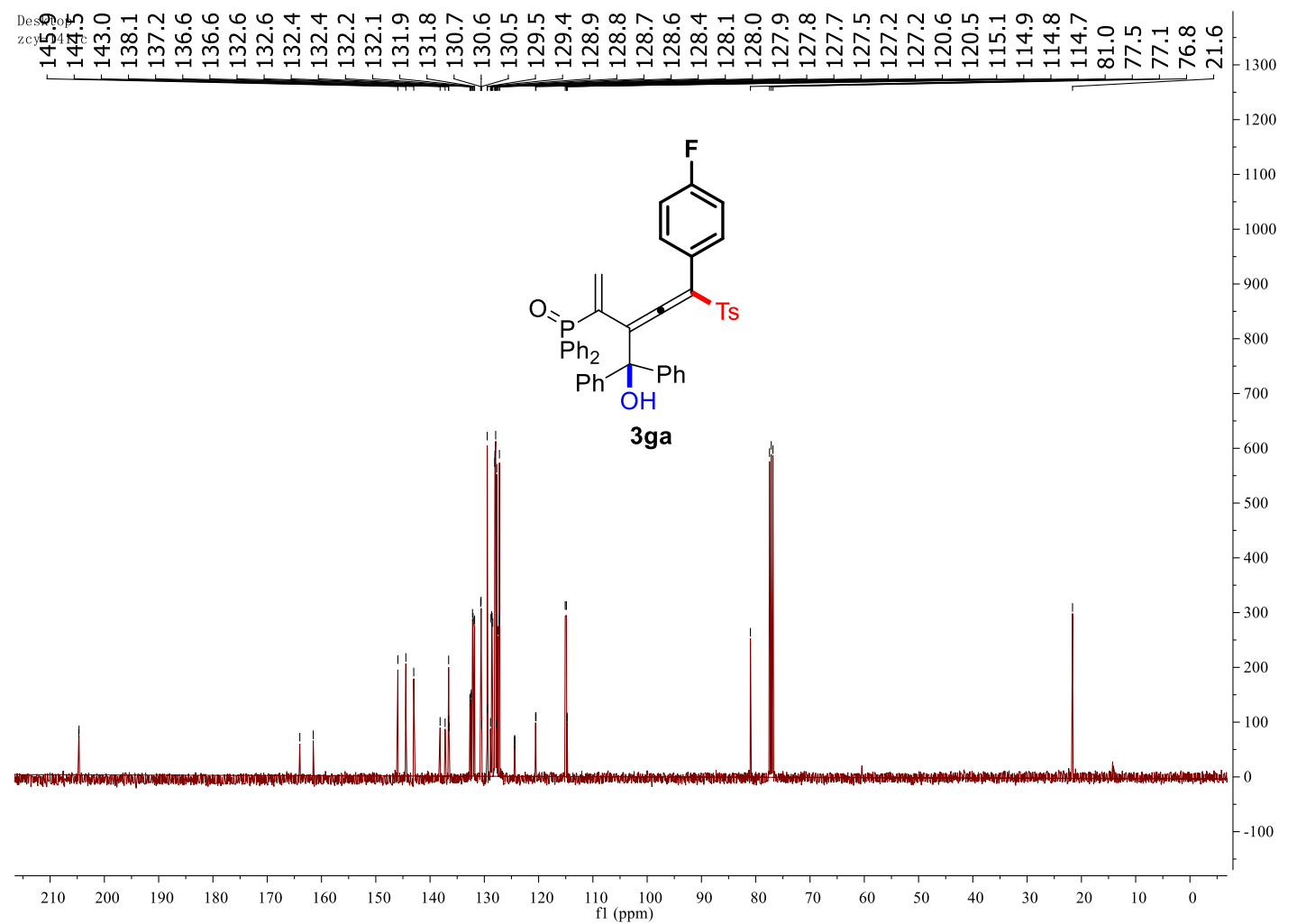

\section{${ }^{13} \mathrm{C}$ NMR $\left(101 \mathrm{MHz}, \mathrm{CDCl}_{3}\right)$ of 3ga}

thenting

$\stackrel{\infty}{\stackrel{\infty}{\sim}}$
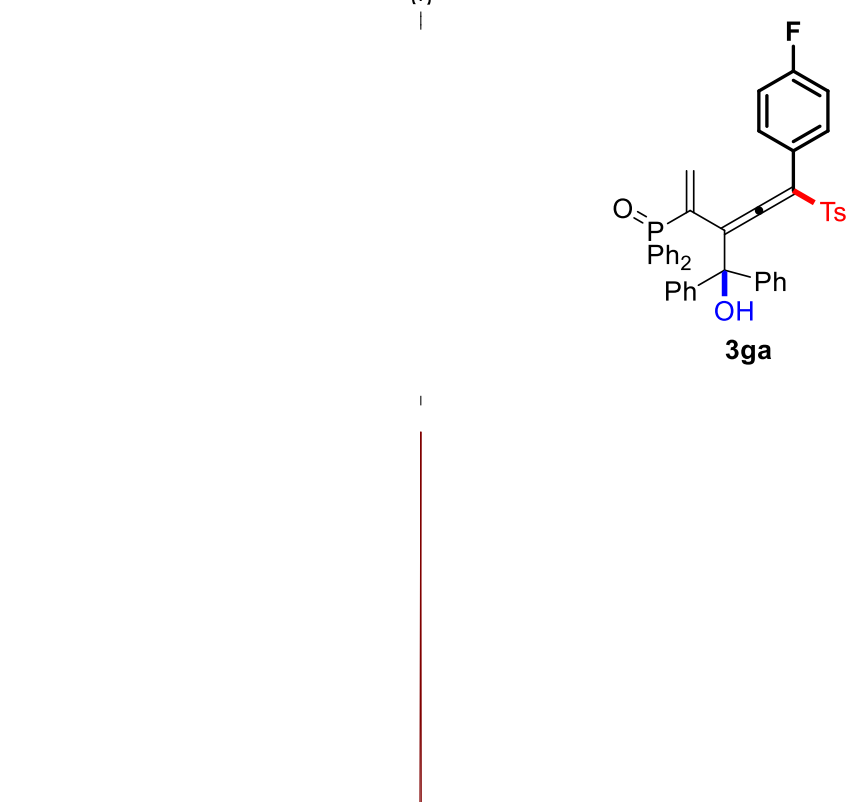$$
80
$$
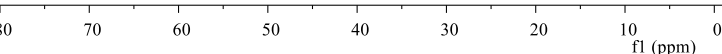

${ }^{31} \mathrm{P}$ NMR (162 MHz, $\left.\mathrm{CDCl}_{3}\right)$ of $3 \mathbf{g a}$ 

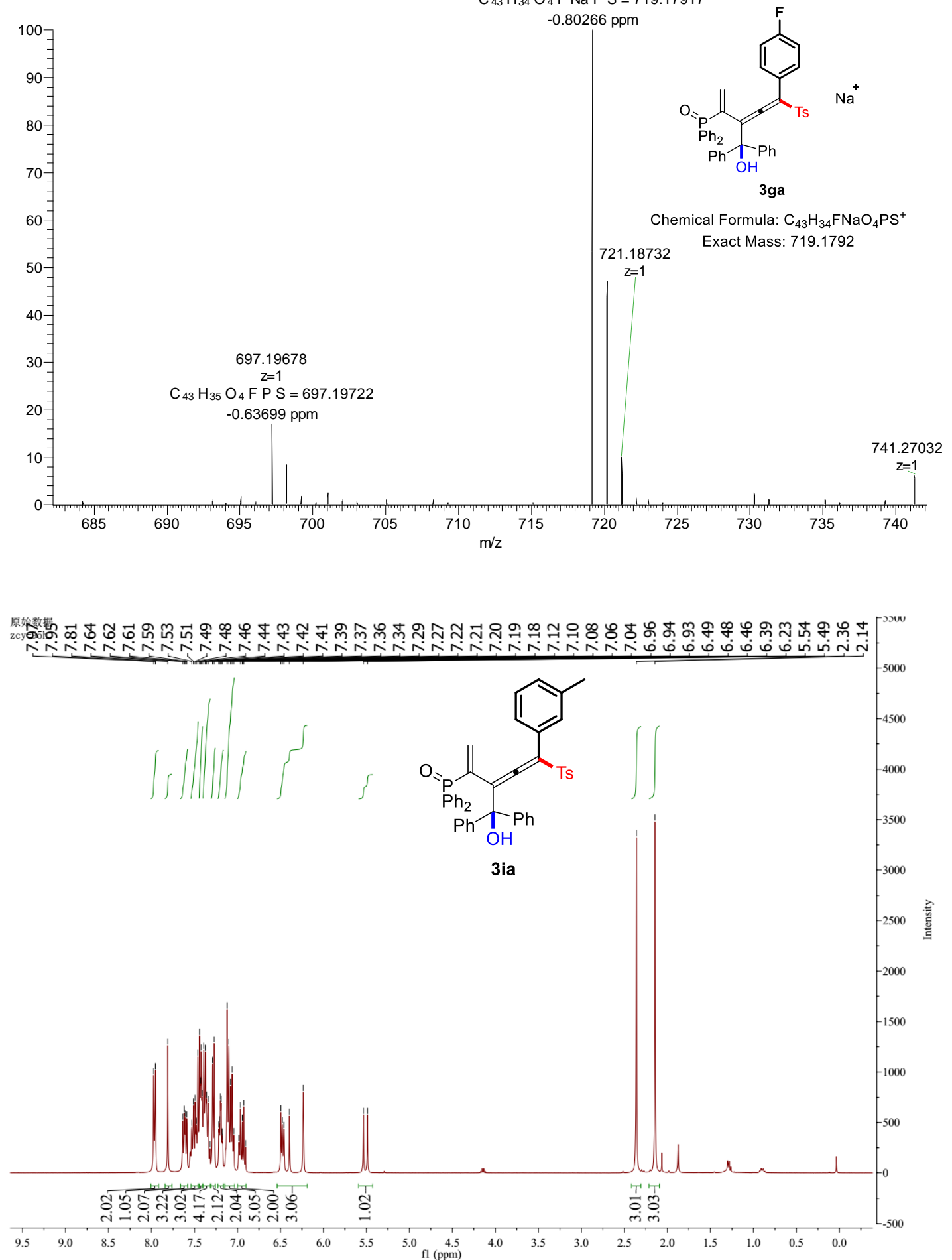

${ }^{1} \mathrm{H}$ NMR $\left(400 \mathrm{MHz}, \mathrm{CDCl}_{3}\right.$ ) of 3ia 


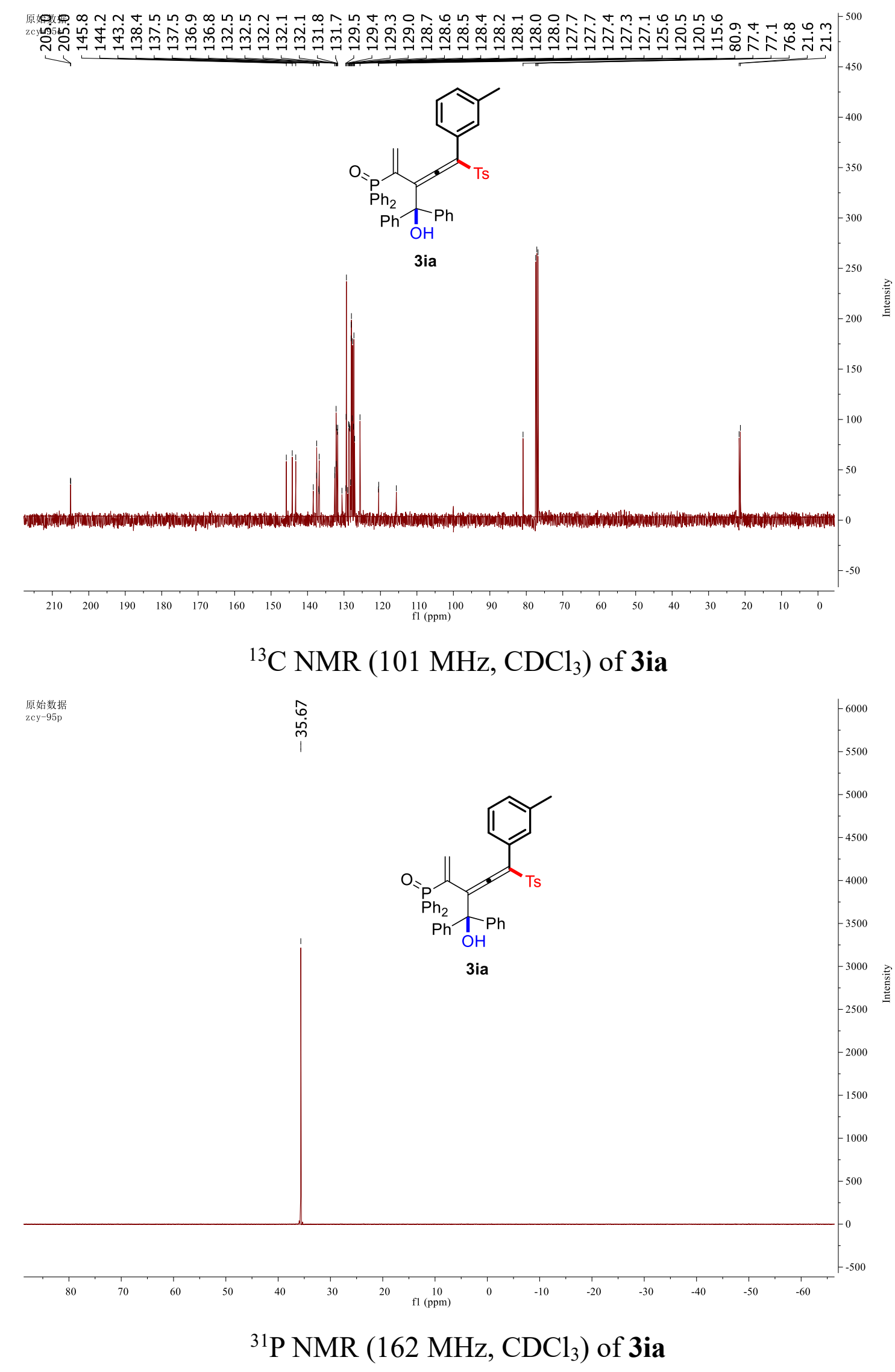


20190506-25 \#31 RT: 0.49 AV: 1 SB: 21 0.01-0.07, 0.77-1.03 NL: 5.19E4
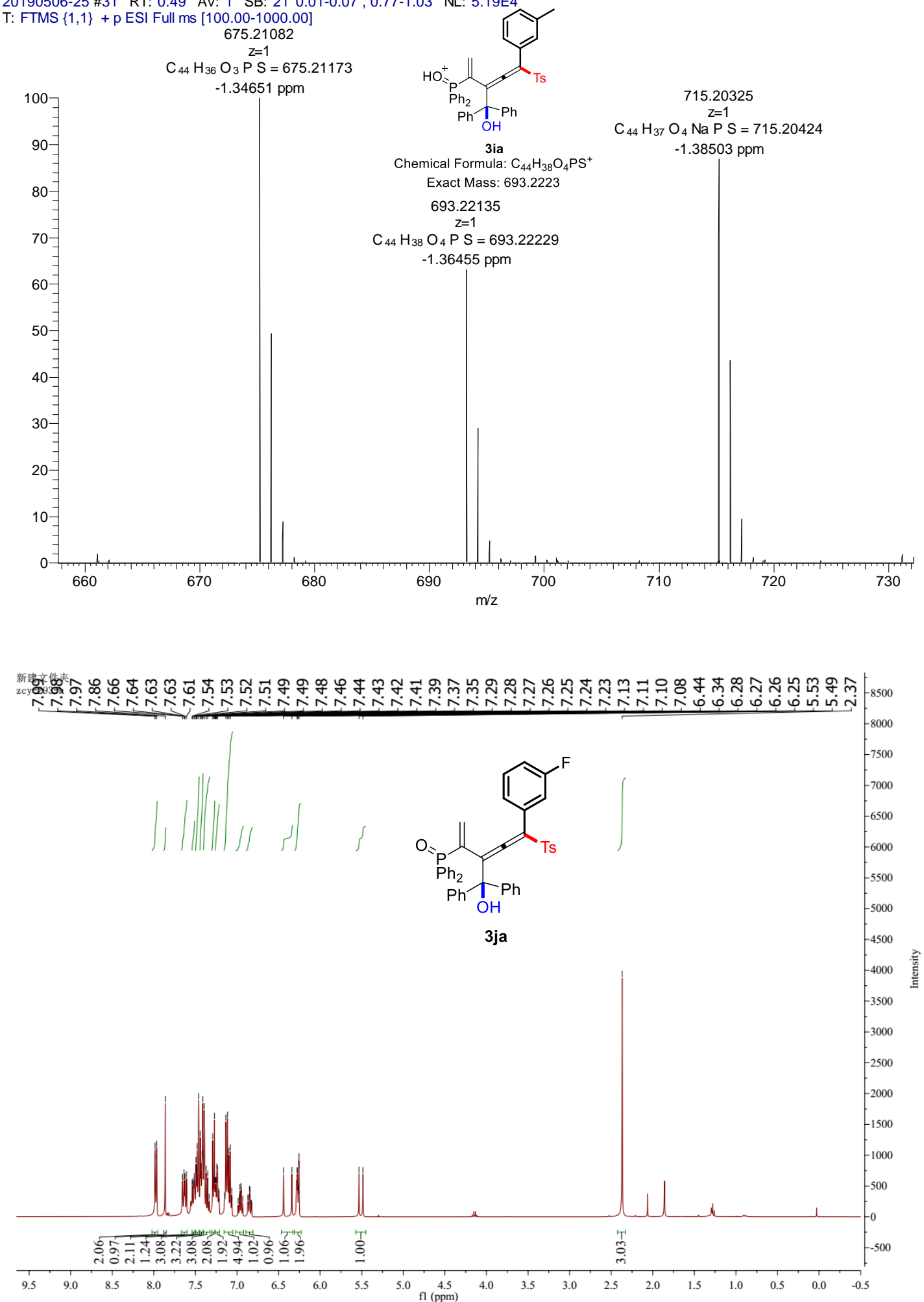

${ }^{1} \mathrm{H} \mathrm{NMR}\left(400 \mathrm{MHz}, \mathrm{CDCl}_{3}\right)$ of $\mathbf{3 j a}$ 


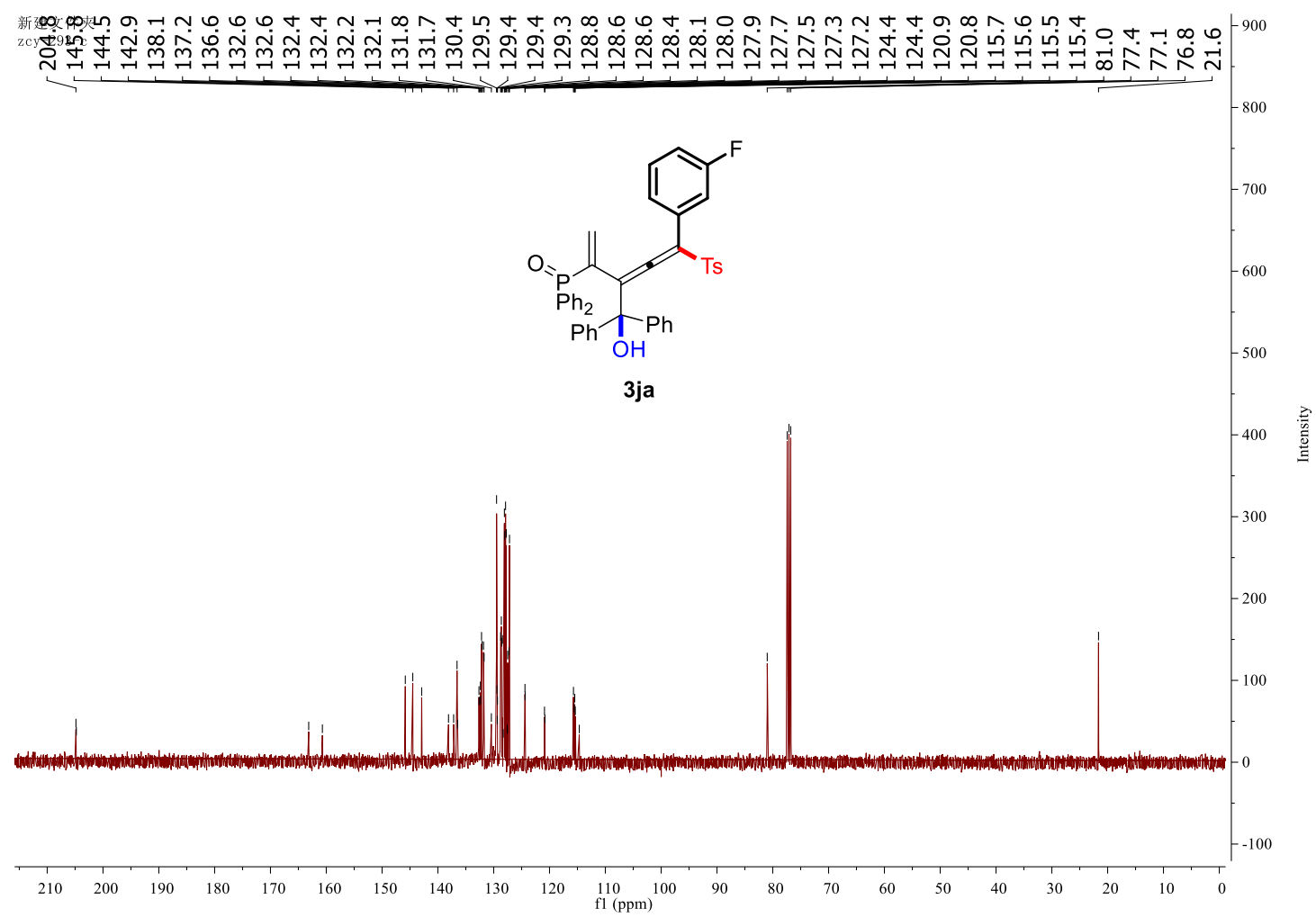

${ }^{13} \mathrm{C}$ NMR (101 MHz, $\left.\mathrm{CDCl}_{3}\right)$ of $\mathbf{3 j a}$

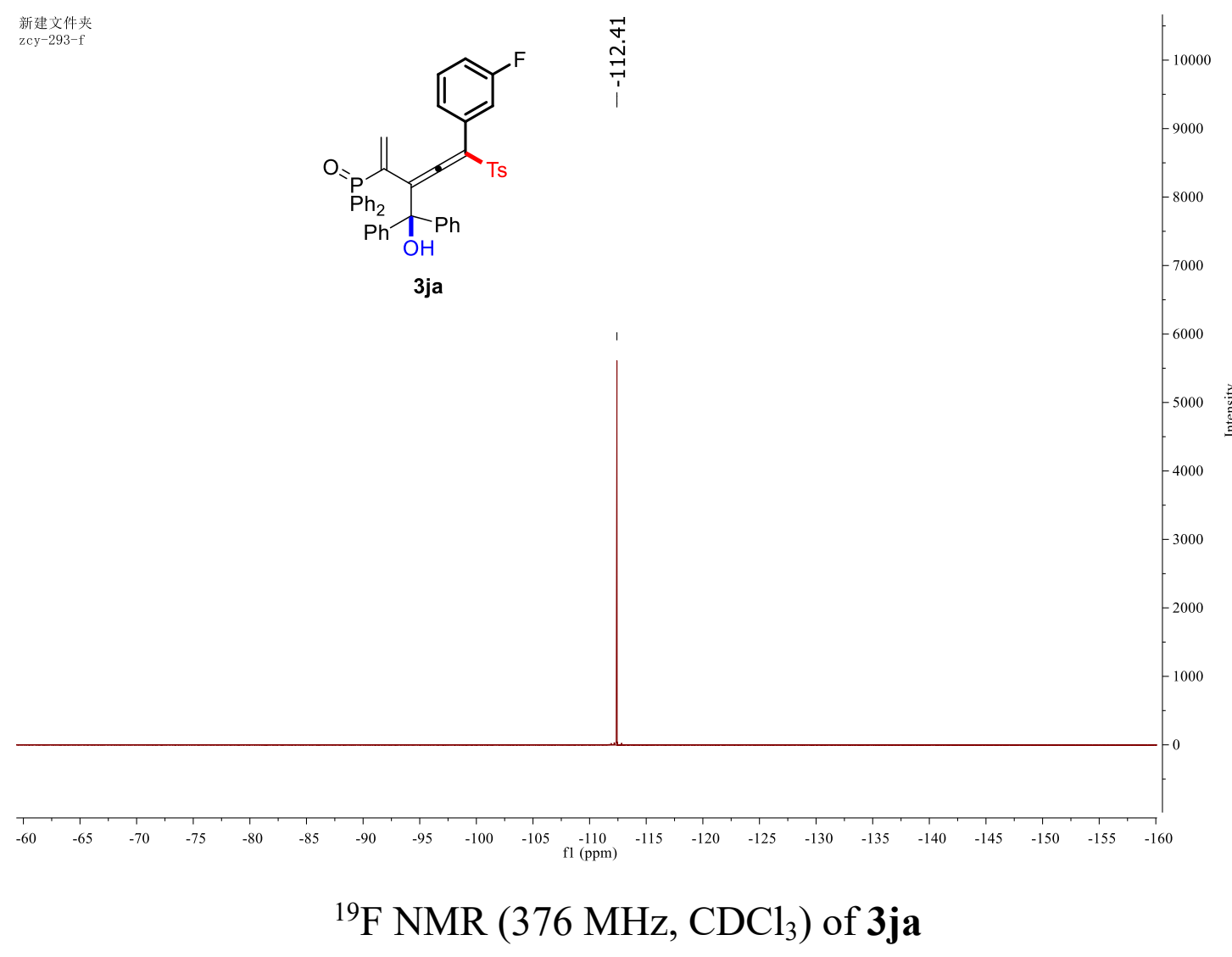




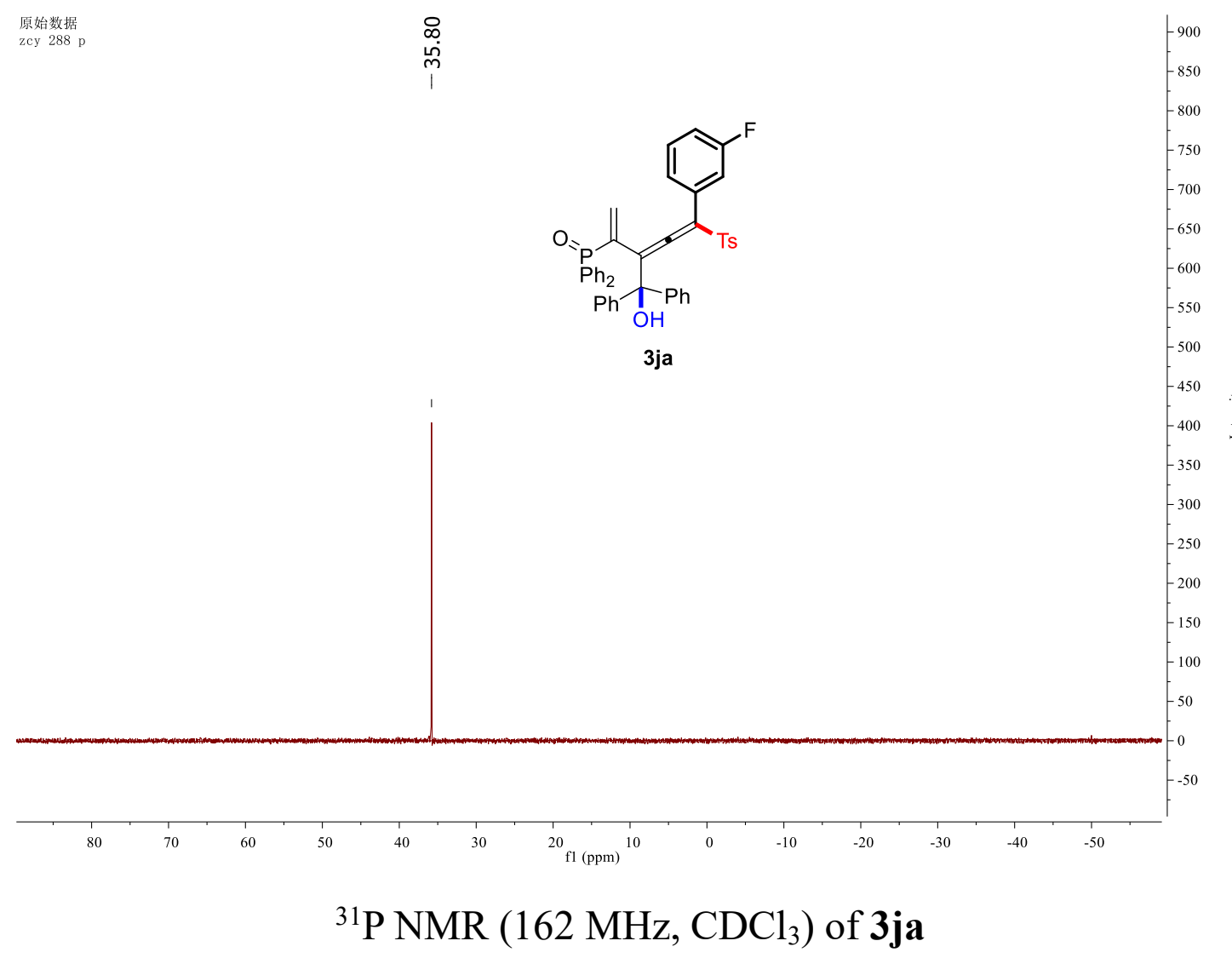

NJNY-5 \#9 RT: 0.11 AV: 1 NL: 4.86E5

T: FTMS $\{1,1\}+$ p ESI Full ms [100.00-1000.00]

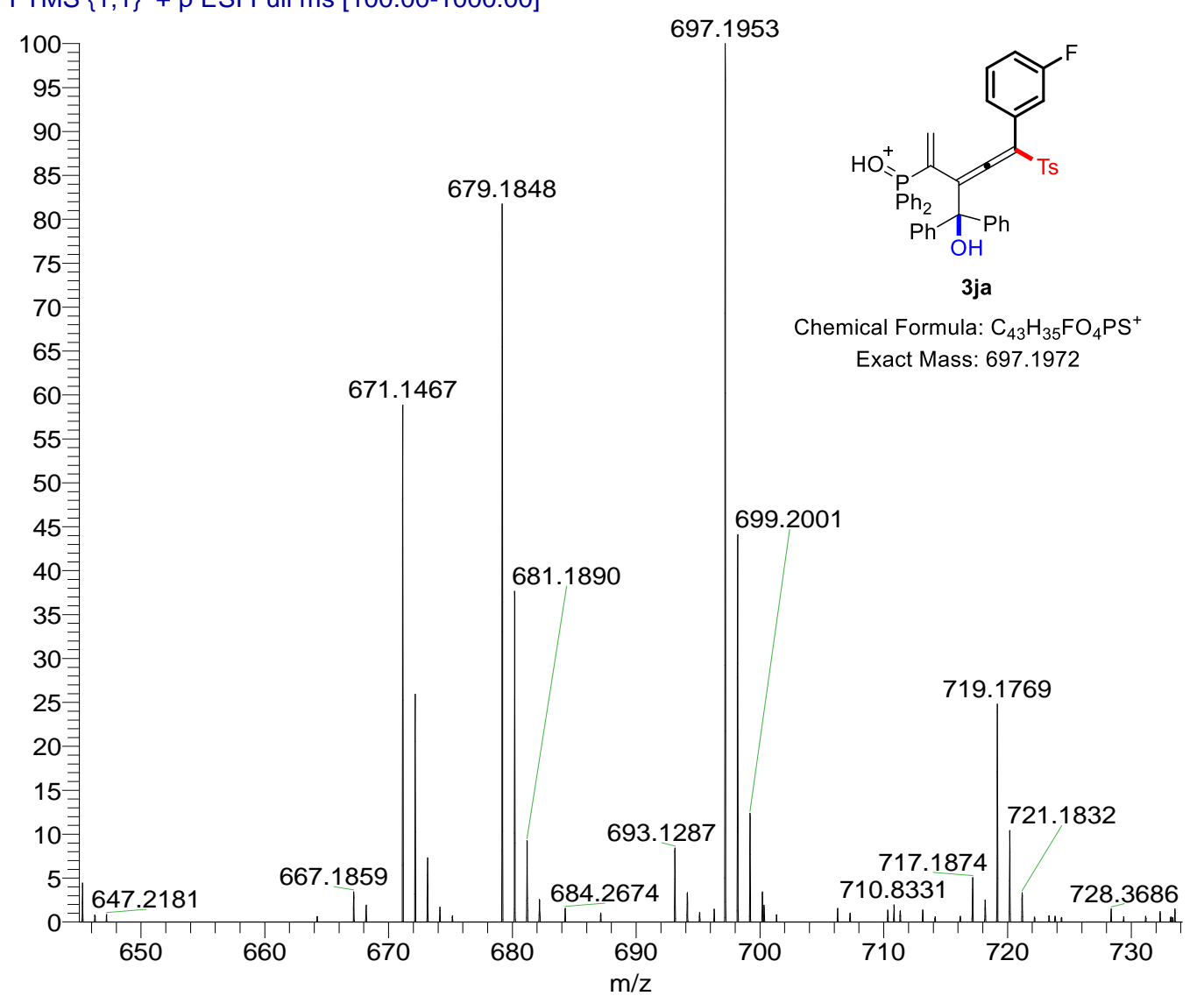



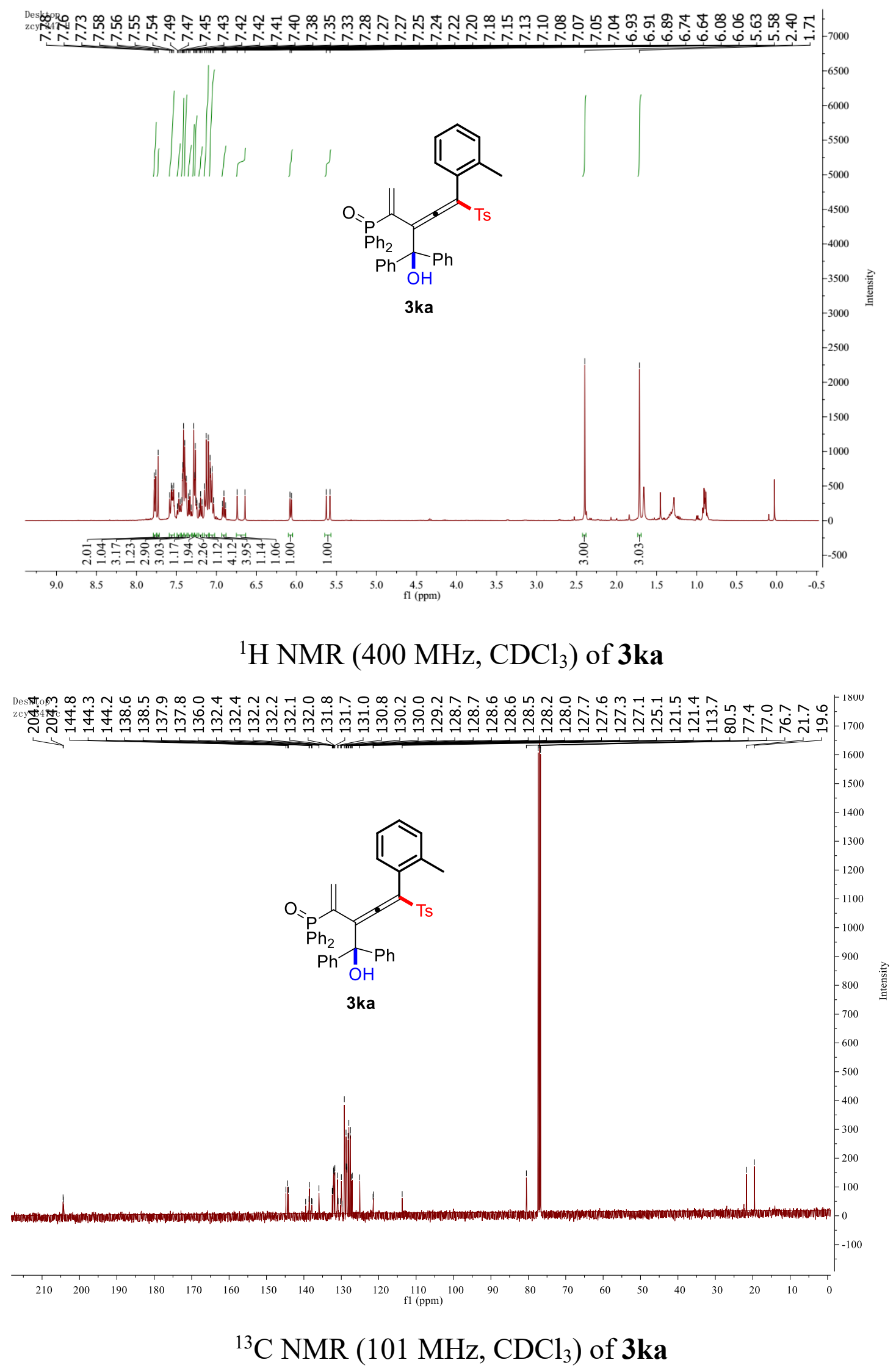


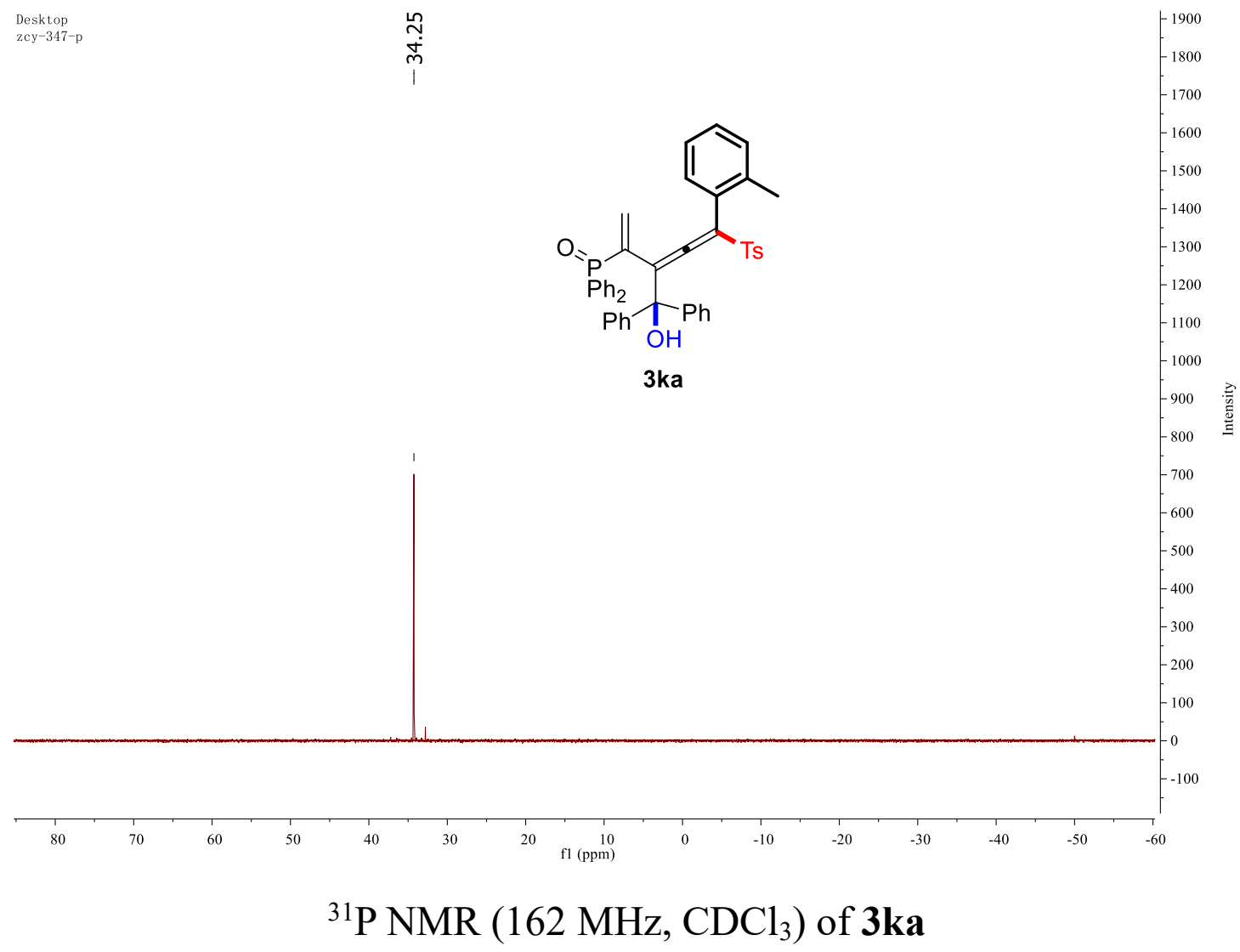

20210326-24 \#9 RT: 0.11 AV: 1 NL: 4.86E6

T: FTMS $\{1,1\}+p$ ESI Full ms [100.00-1000.00]

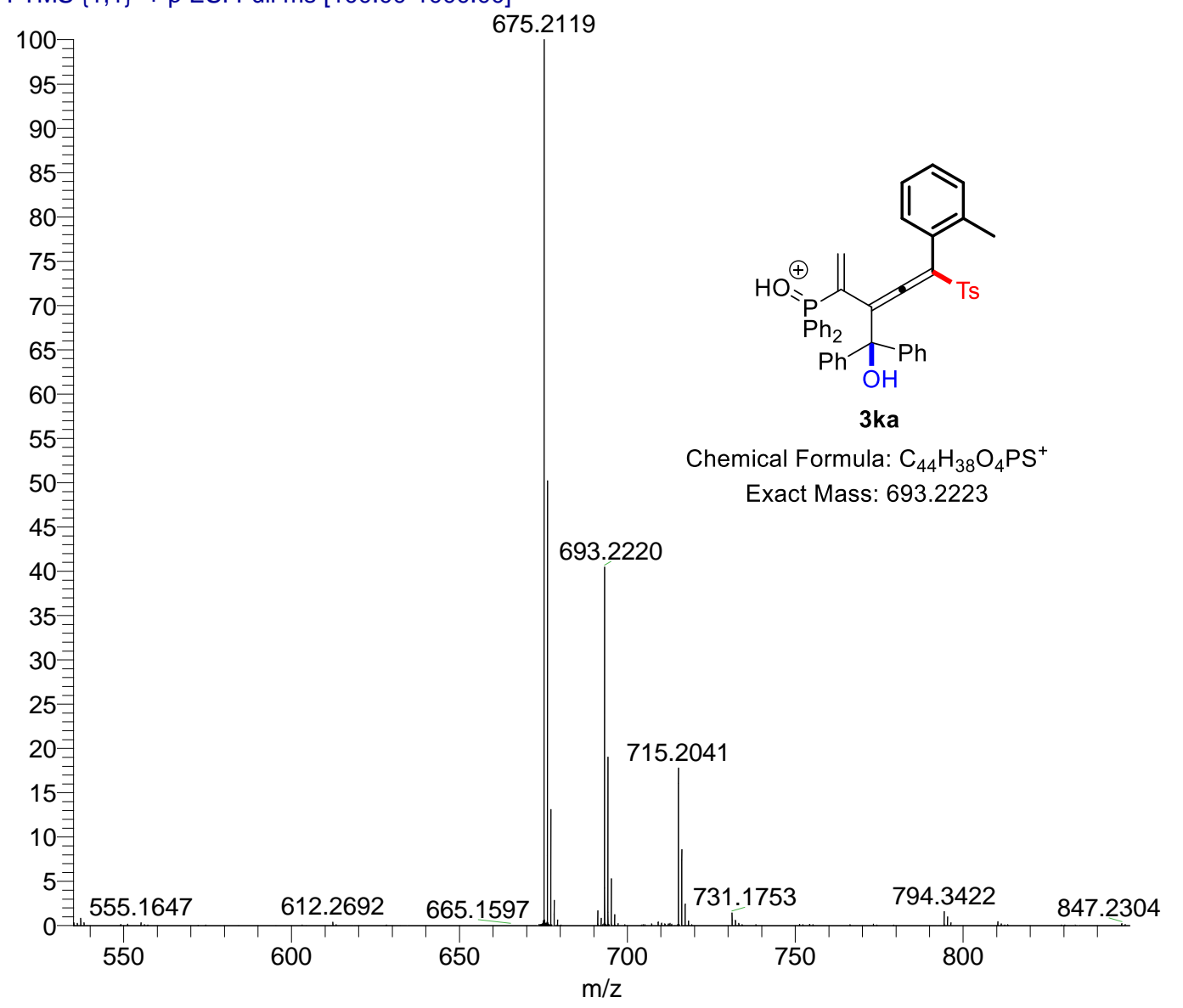

127 


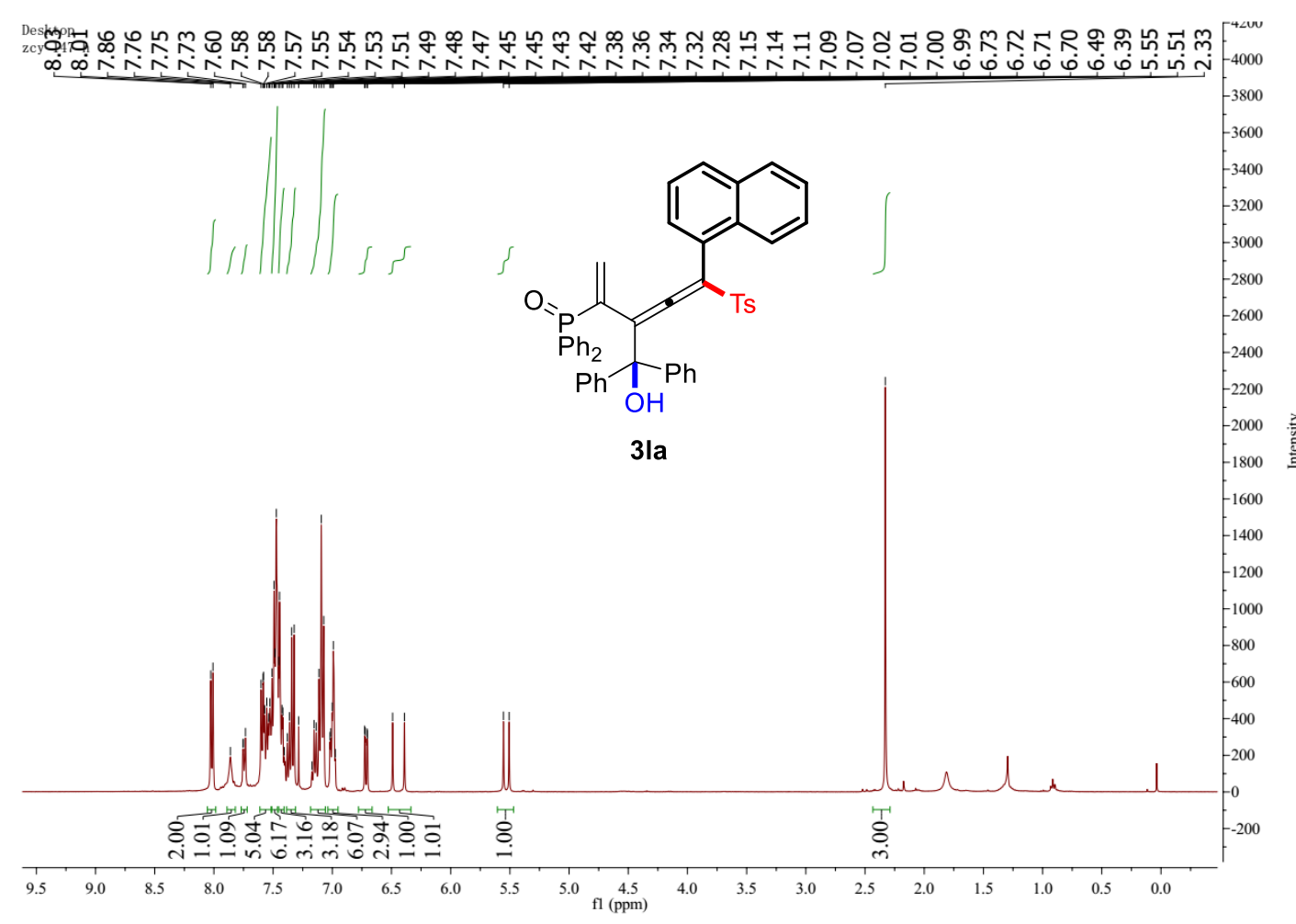

${ }^{1} \mathrm{H}$ NMR $\left(400 \mathrm{MHz}, \mathrm{CDCl}_{3}\right)$ of 3 la

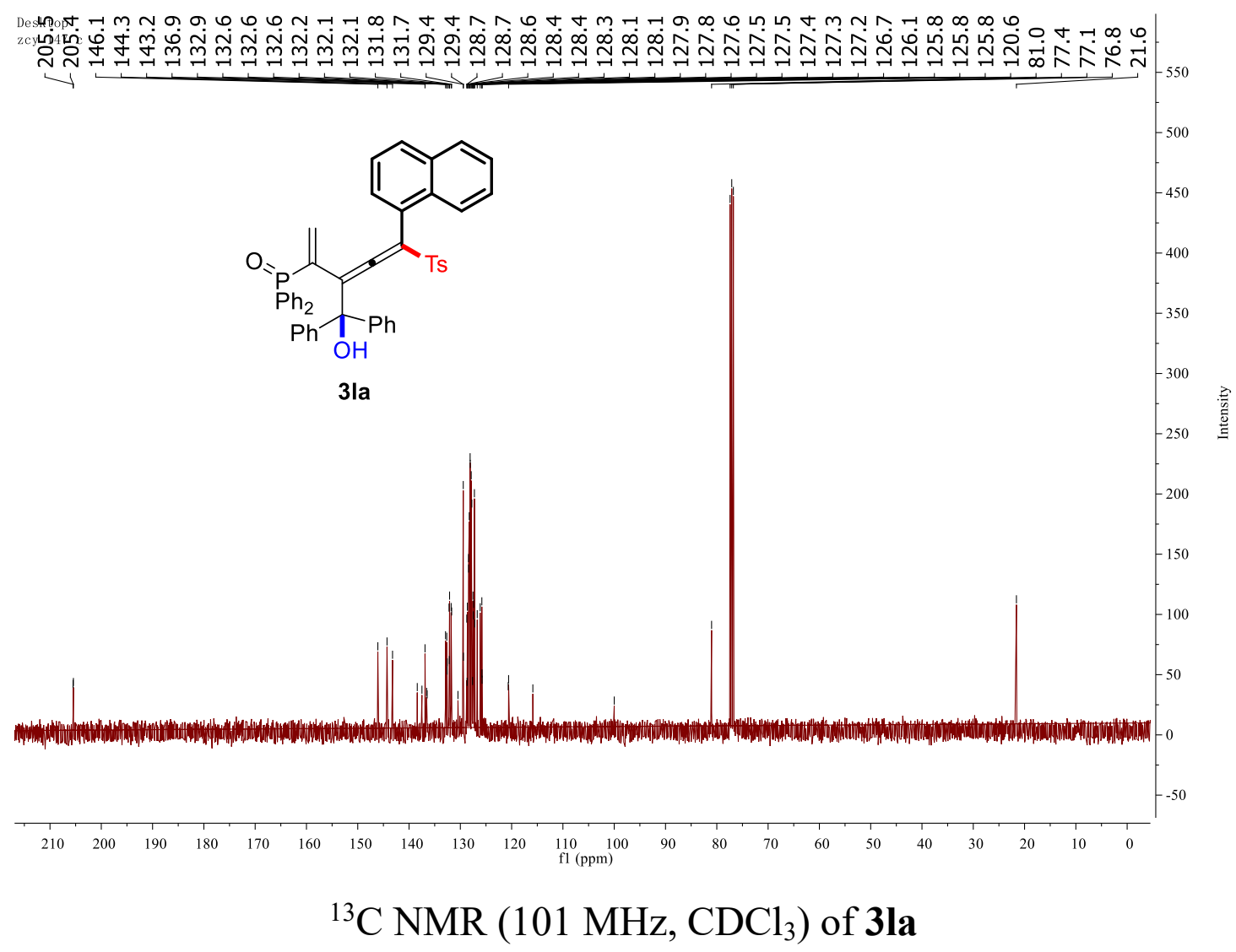




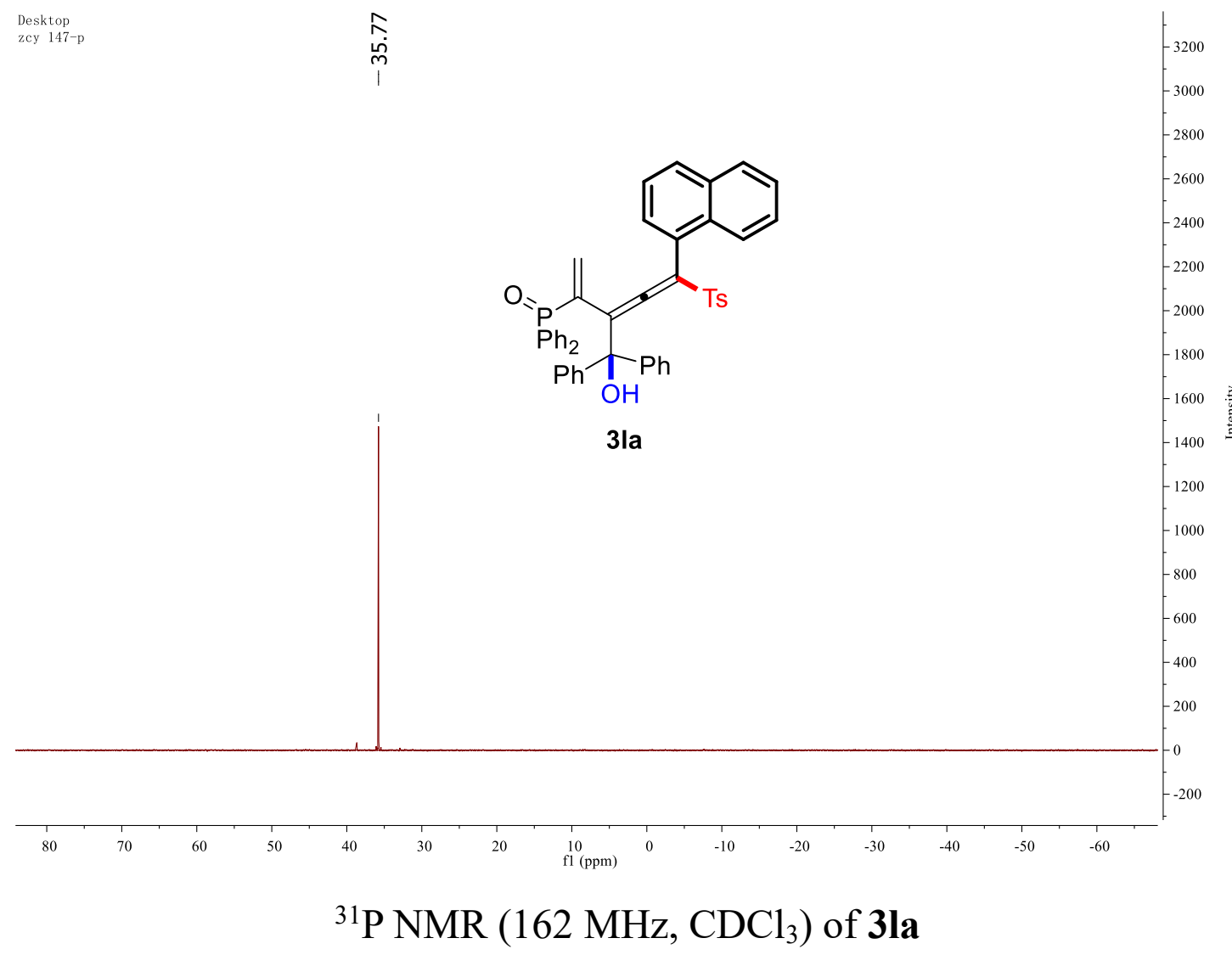

20190708-11 \#9 RT: 0.13 AV: 1 NL: 5.21E6

T: FTMS $\{1,1\}+p$ ESI Full ms [100.00-1000.00]

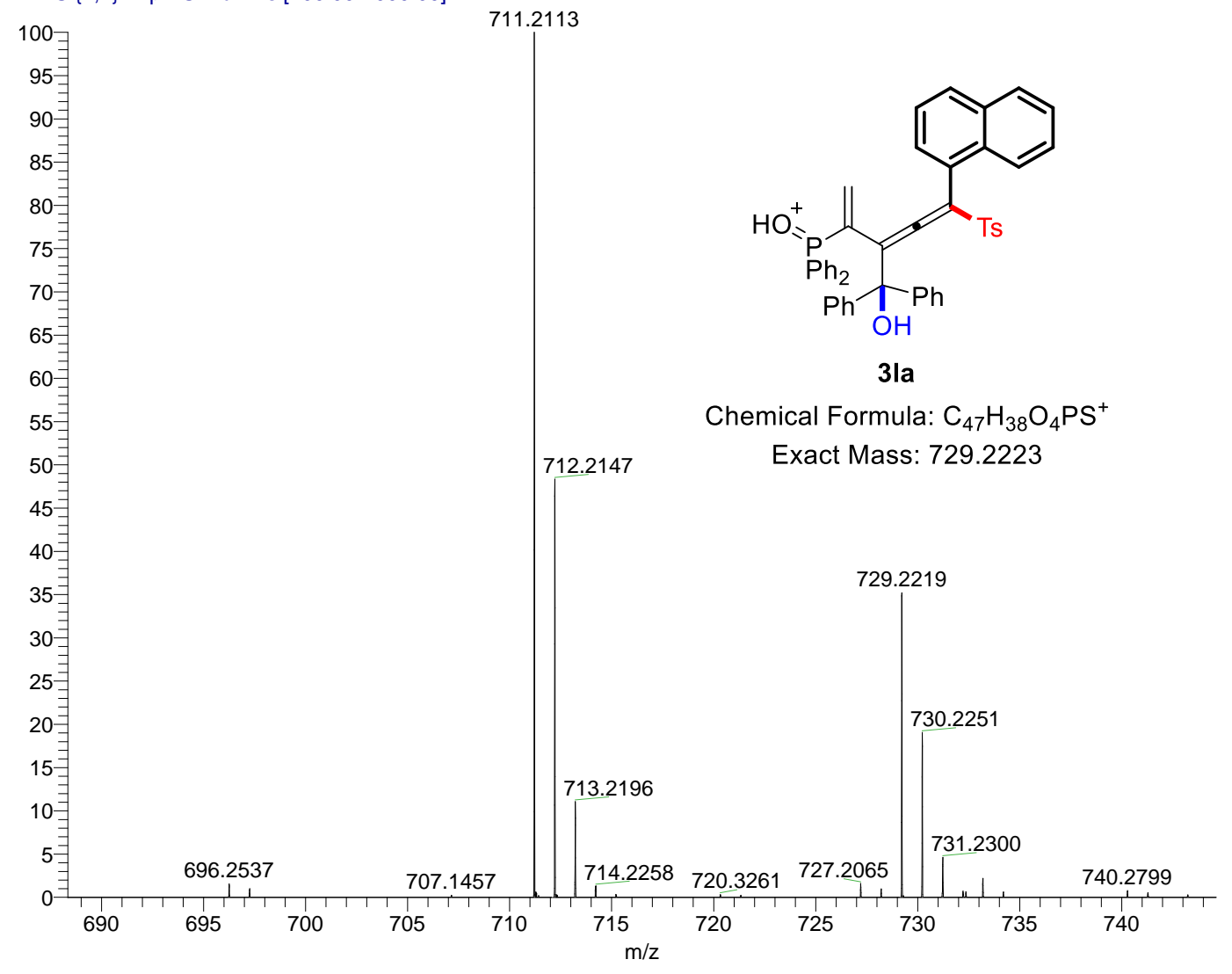




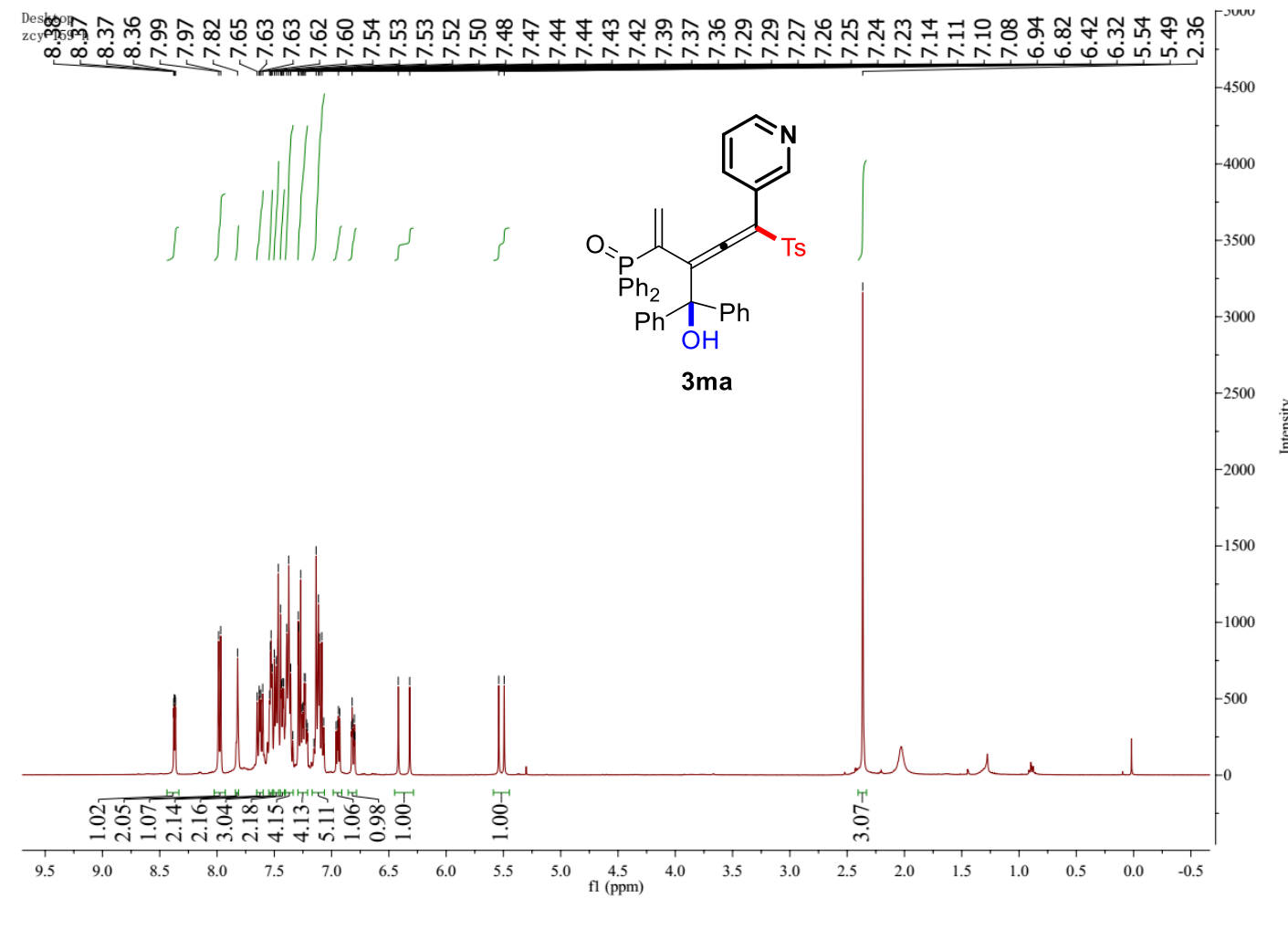

${ }^{1} \mathrm{H}$ NMR (400 MHz, $\mathrm{CDCl}_{3}$ ) of $\mathbf{3 m a}$

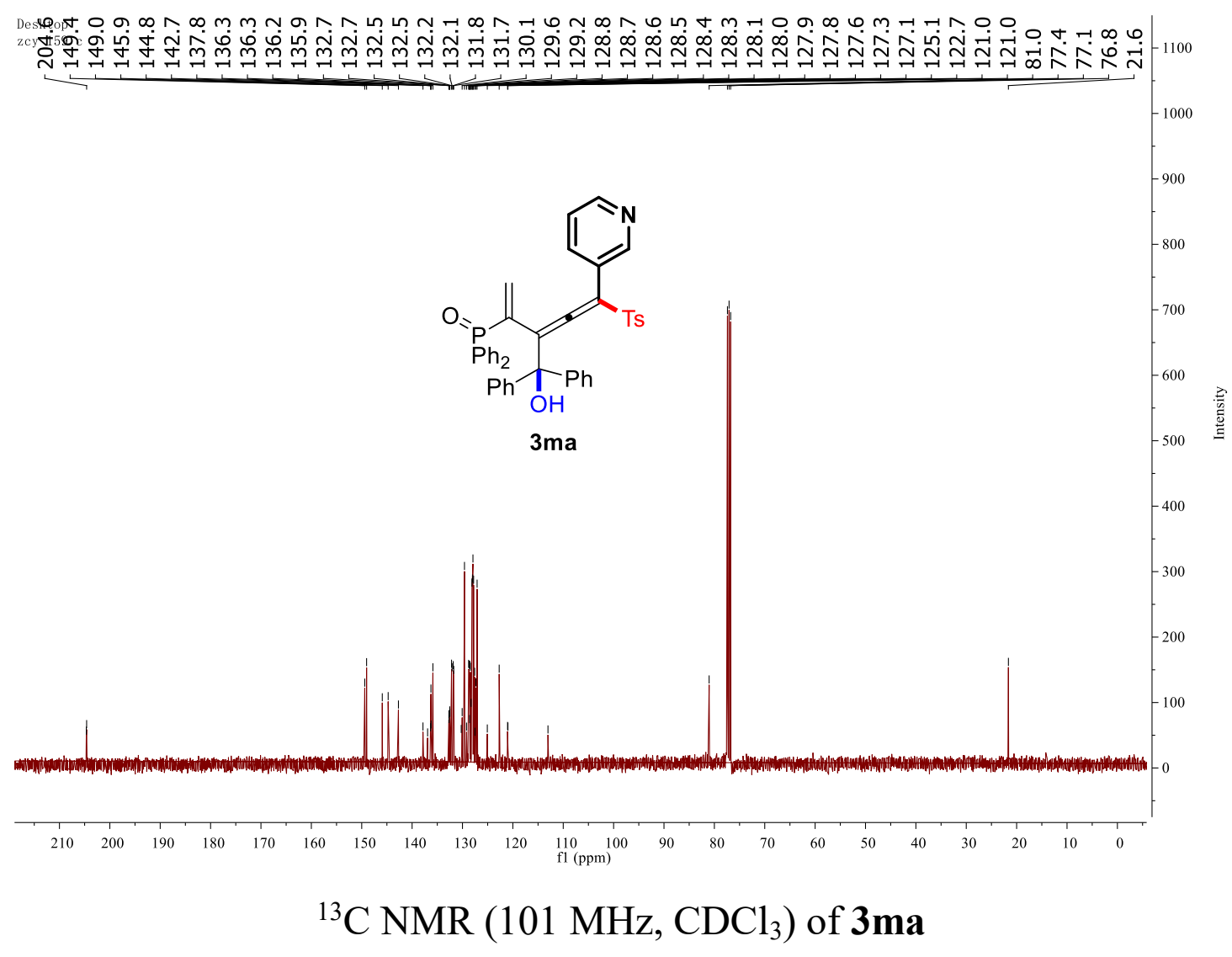




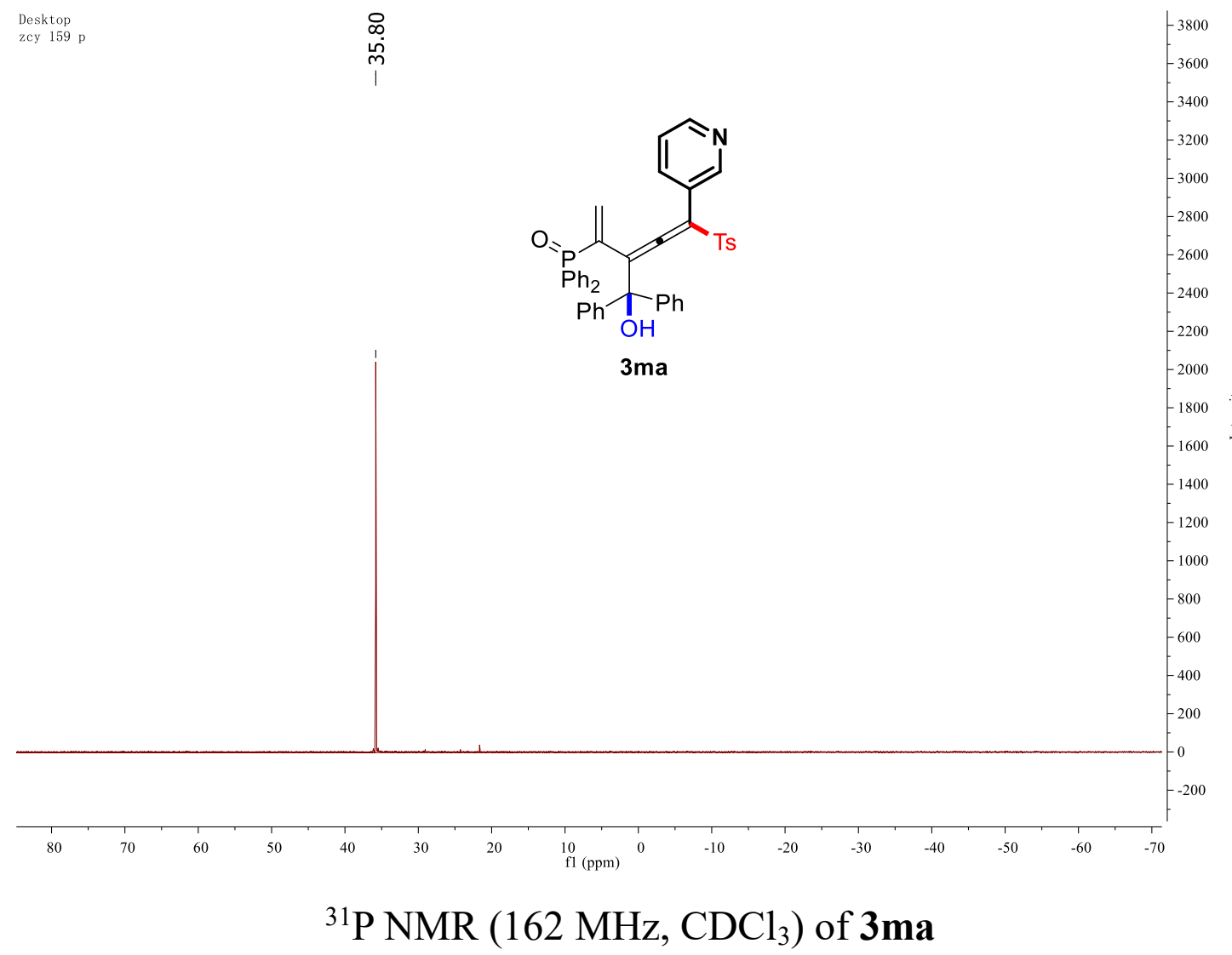

Spectrum from 2.wiff (sample 1) - Sample002, Experiment 1, +TOF MS (50 - 1000) from 0.085 min

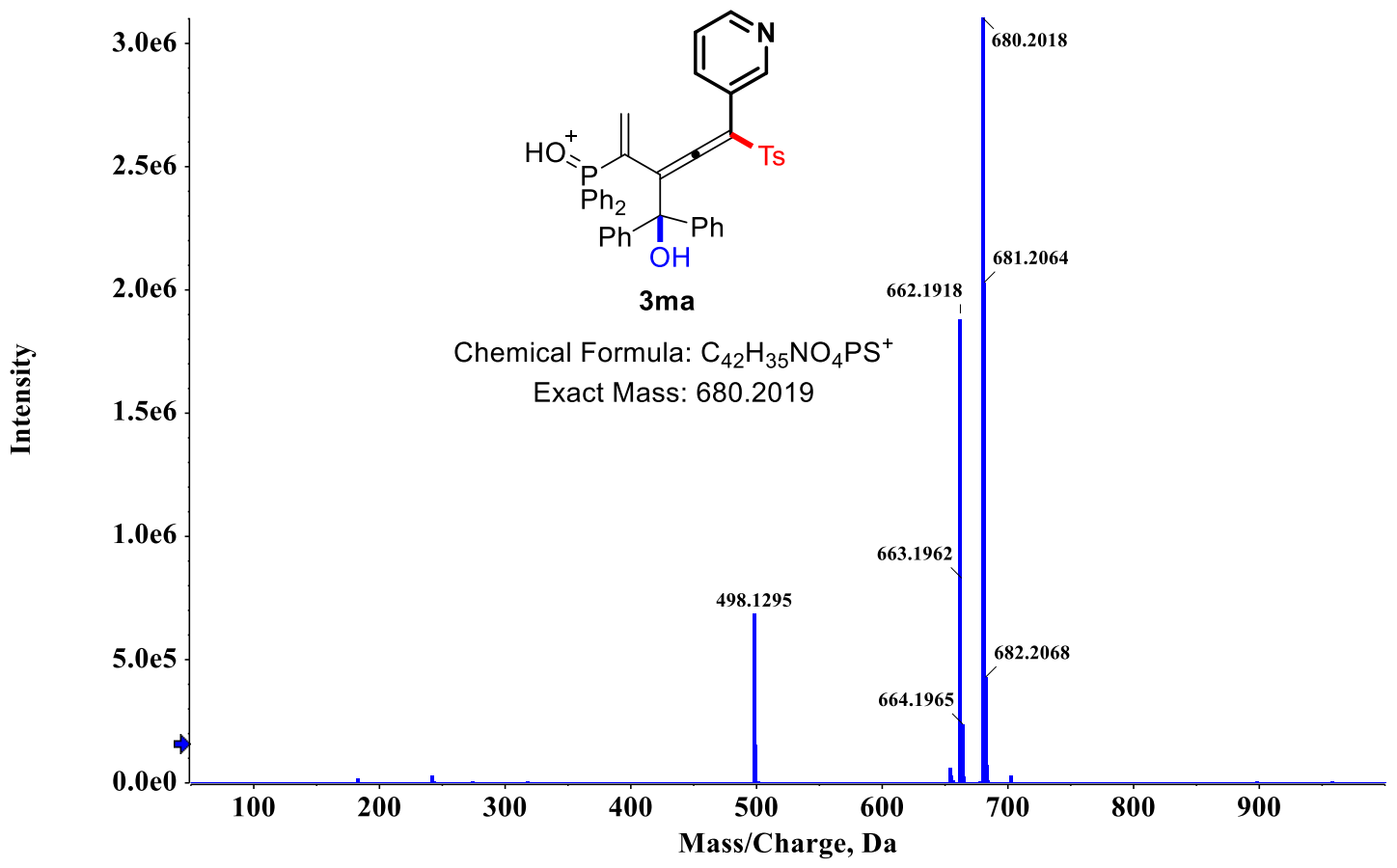




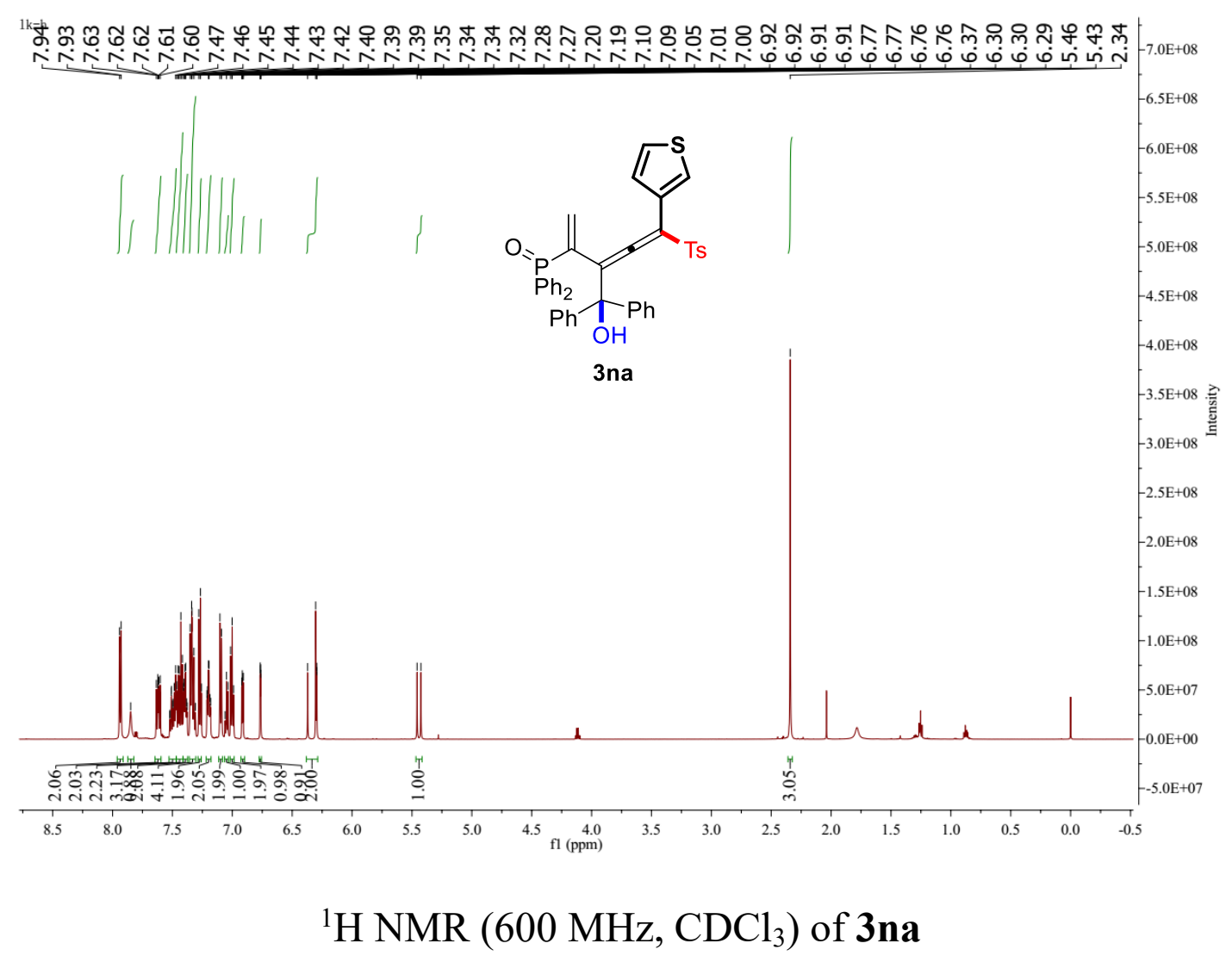

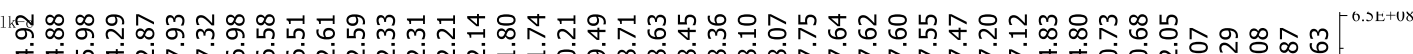

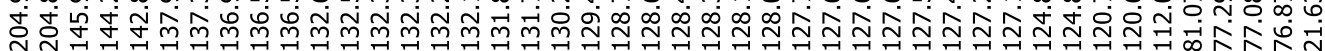

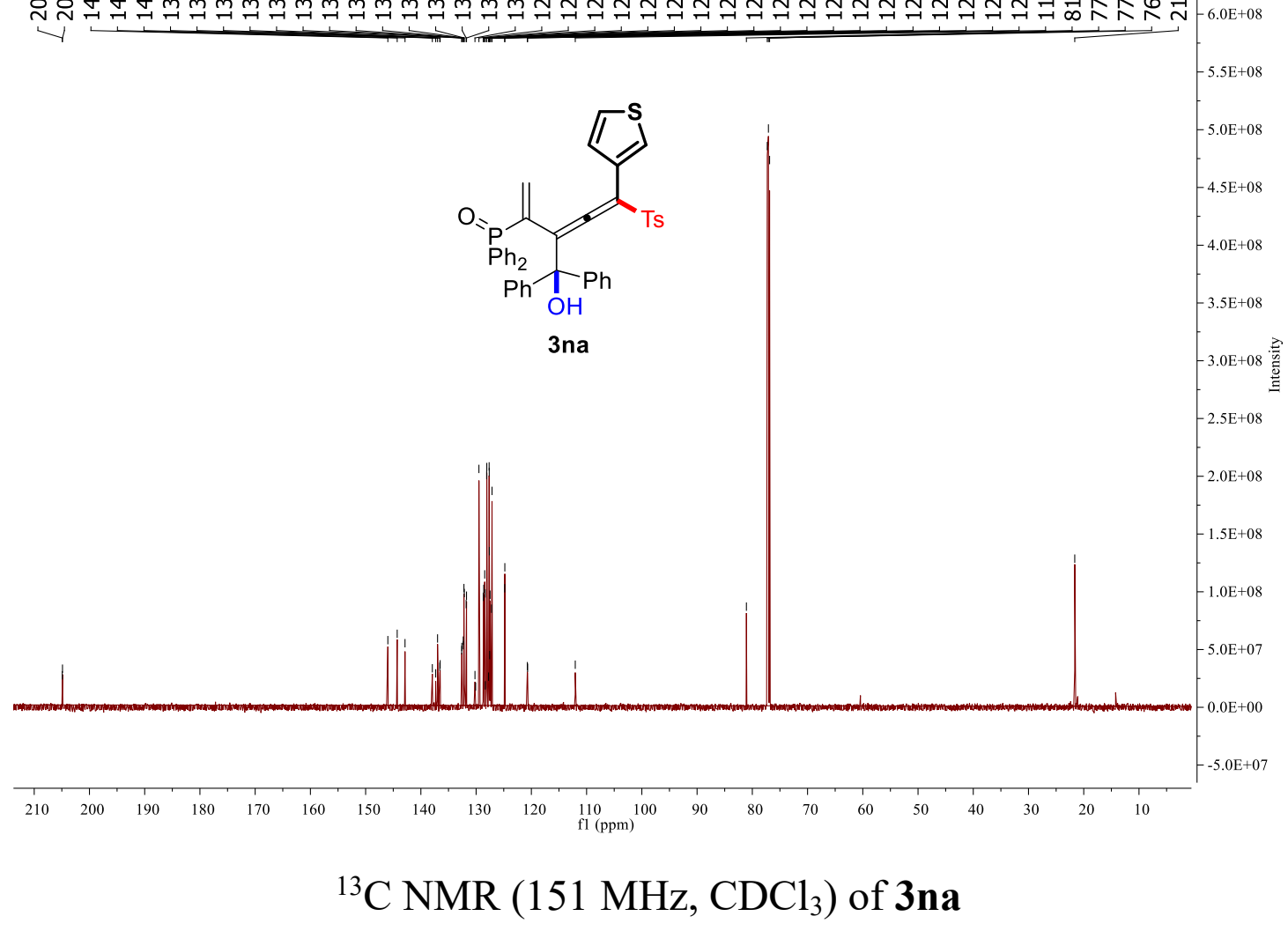




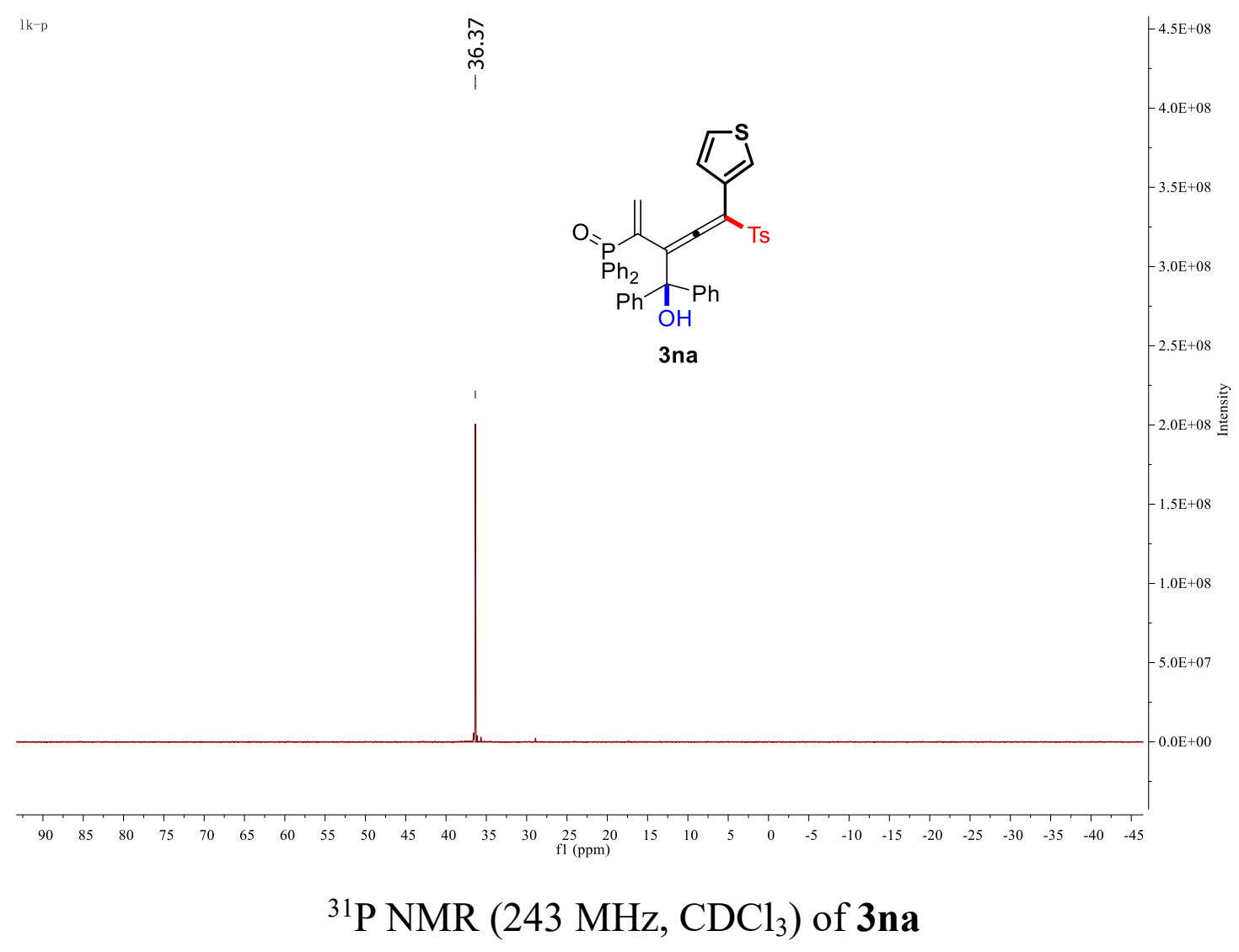

20200827-12 \#15 RT: 0.22 AV: 1 NL: 5.13E6

T: FTMS $\{1,1\}+p$ ESI Full ms [100.00-1000.00]

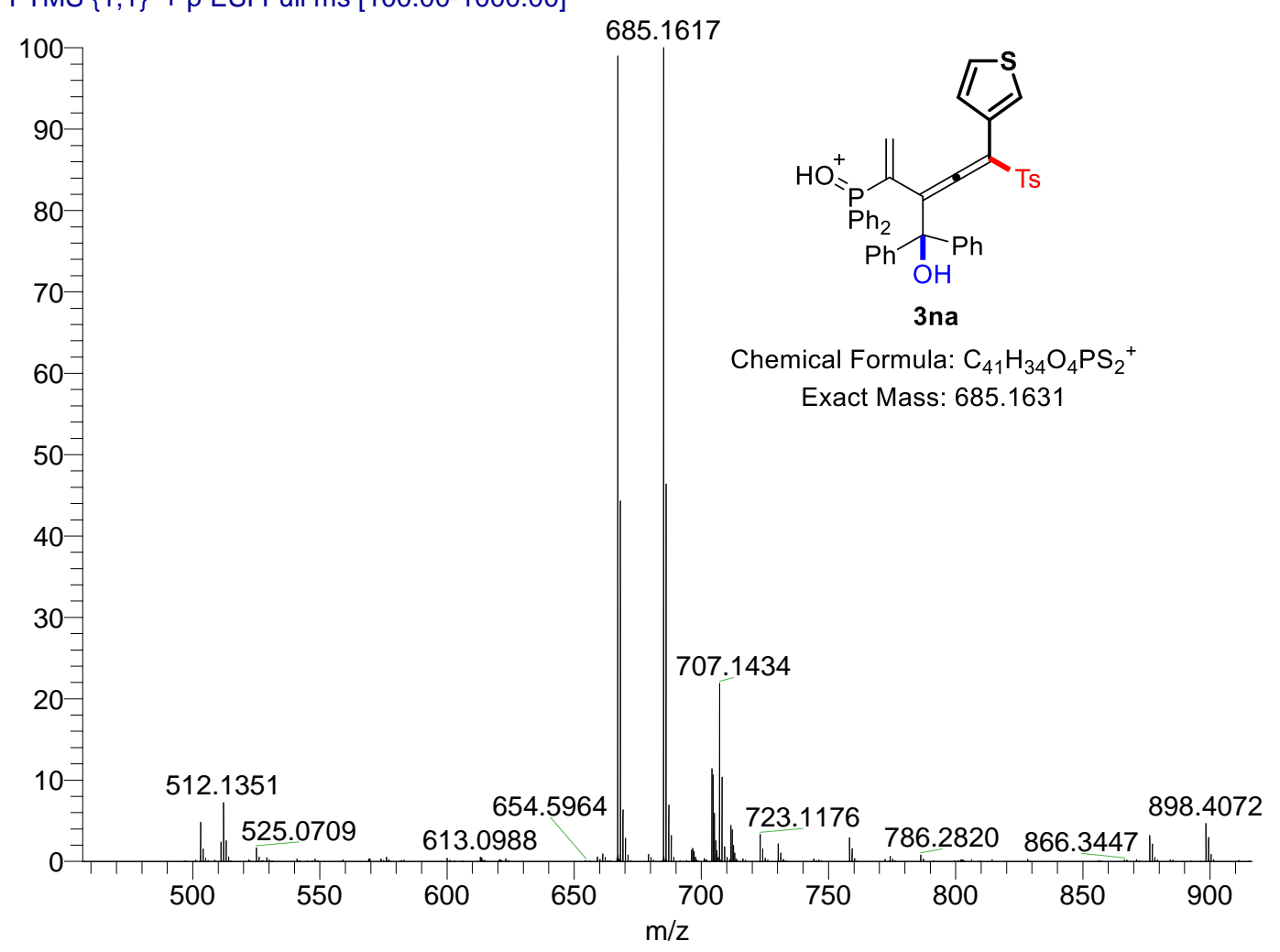




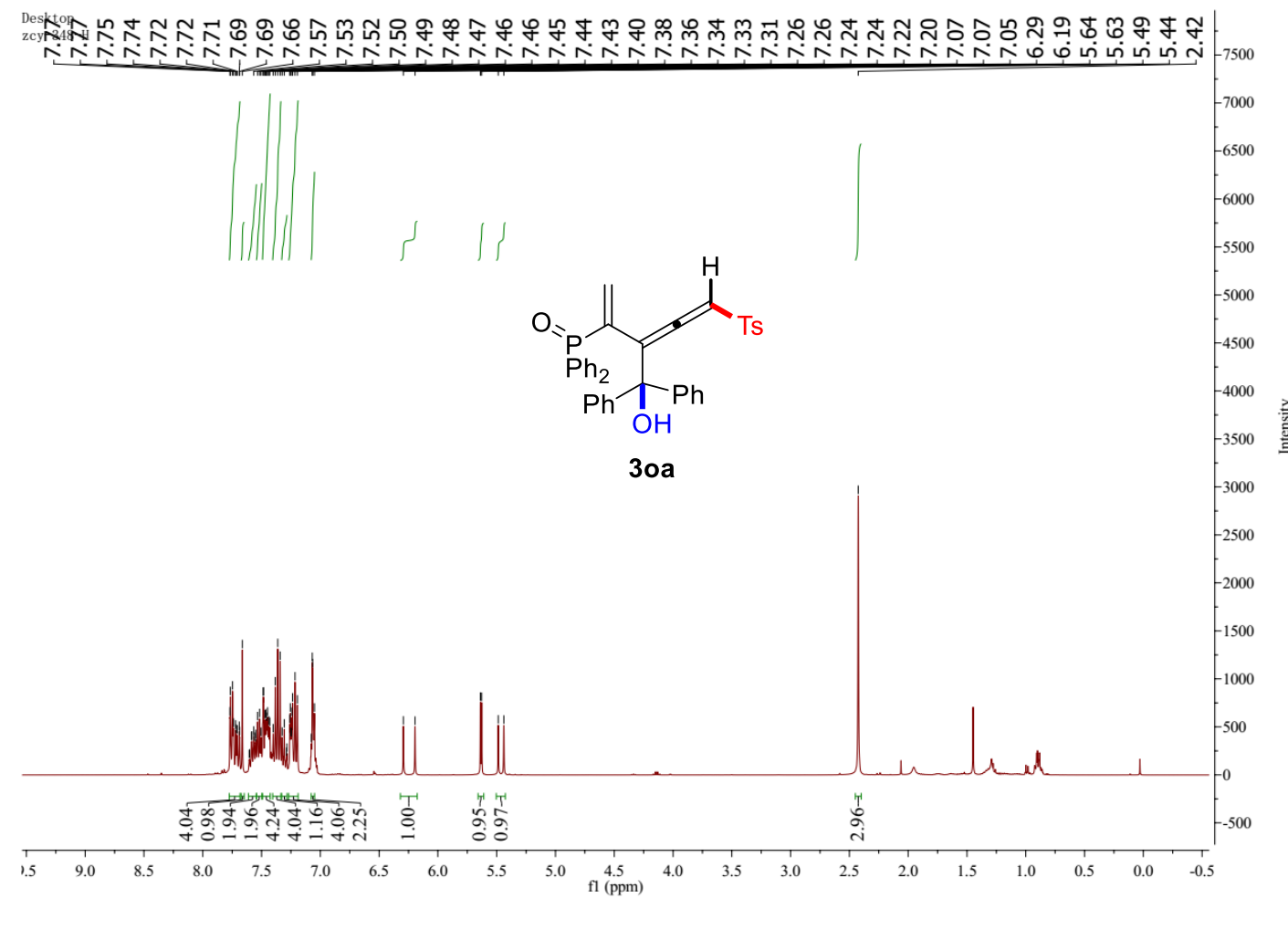

\section{${ }^{1} \mathrm{H} \mathrm{NMR}\left(400 \mathrm{MHz}, \mathrm{CDCl}_{3}\right)$ of $\mathbf{3 o a}$}

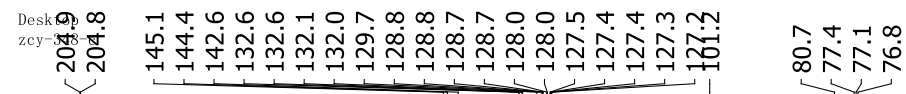

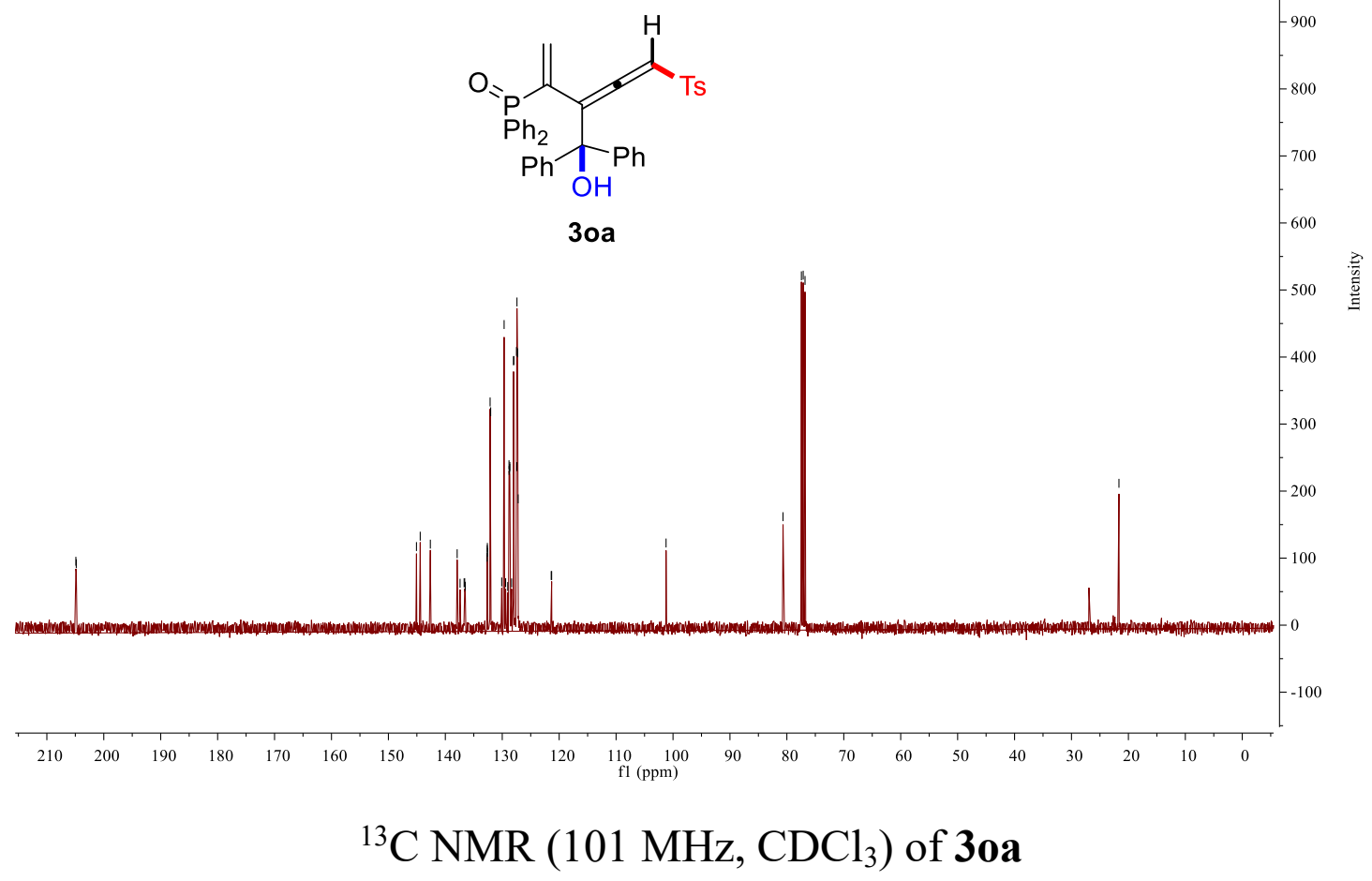




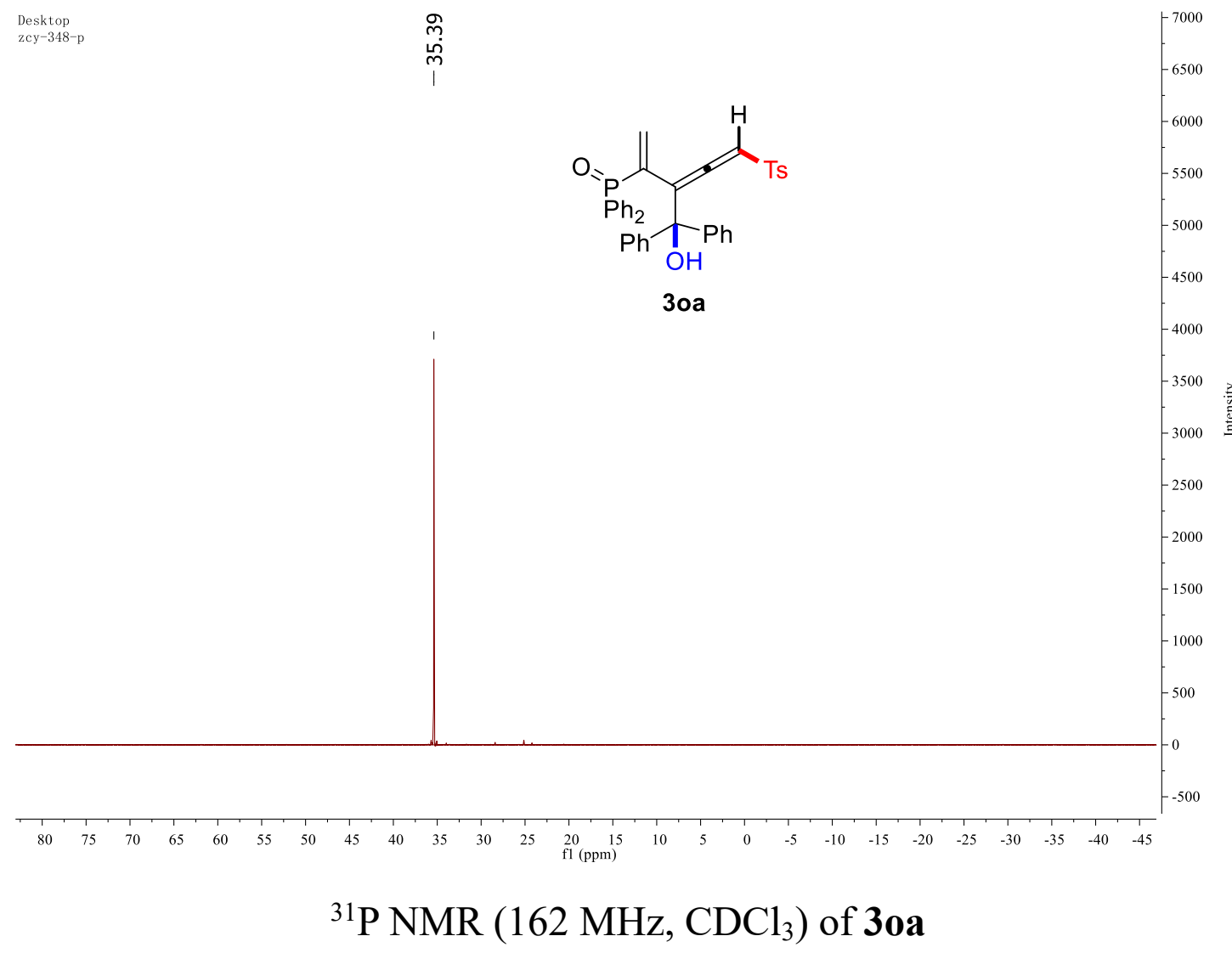

20210326-25 \#9 RT: 0.11 AV: 1 NL: 6.00E6

T: FTMS $\{1,1\}+p$ ESI Full ms [100.00-1000.00]

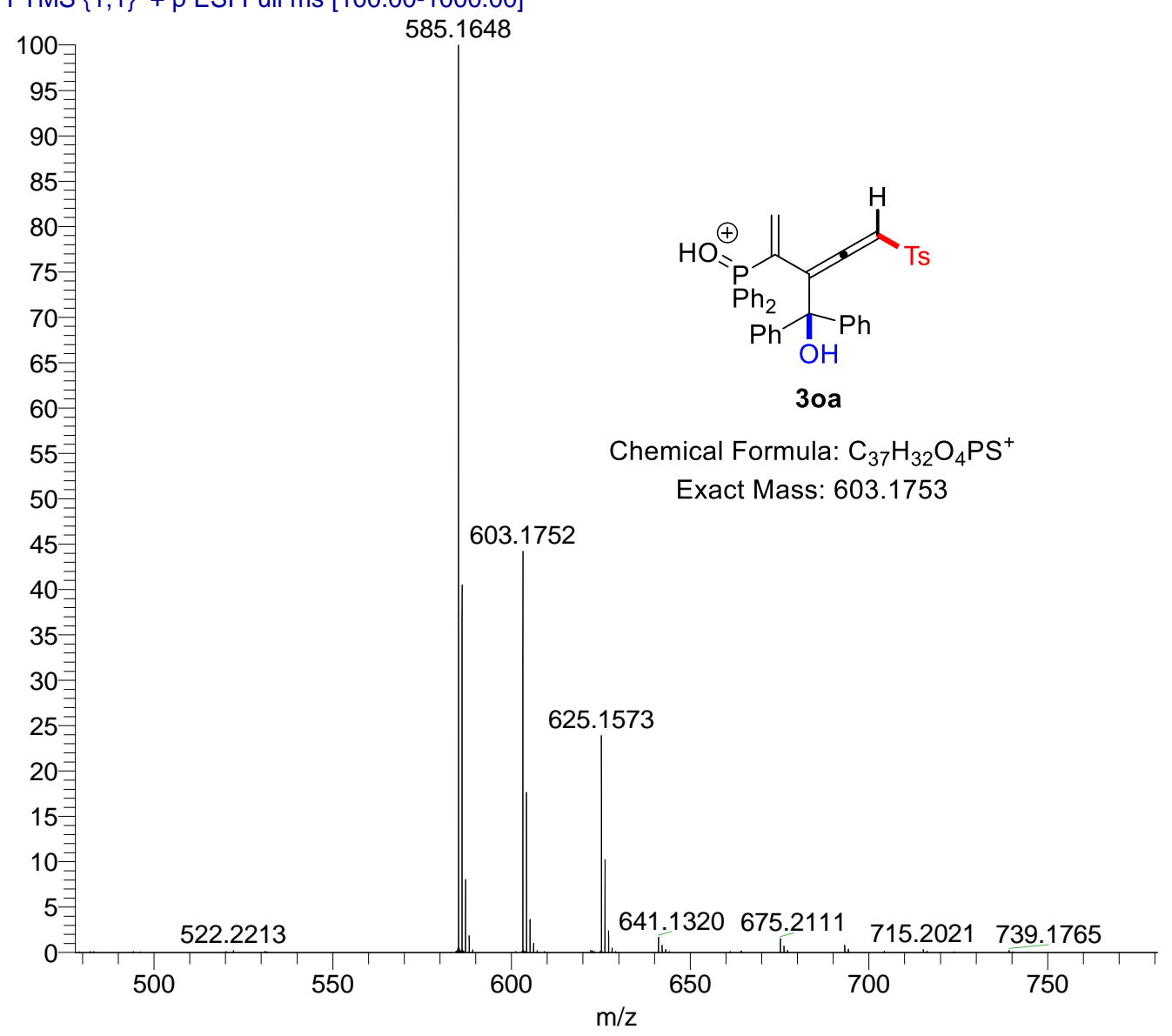

135 


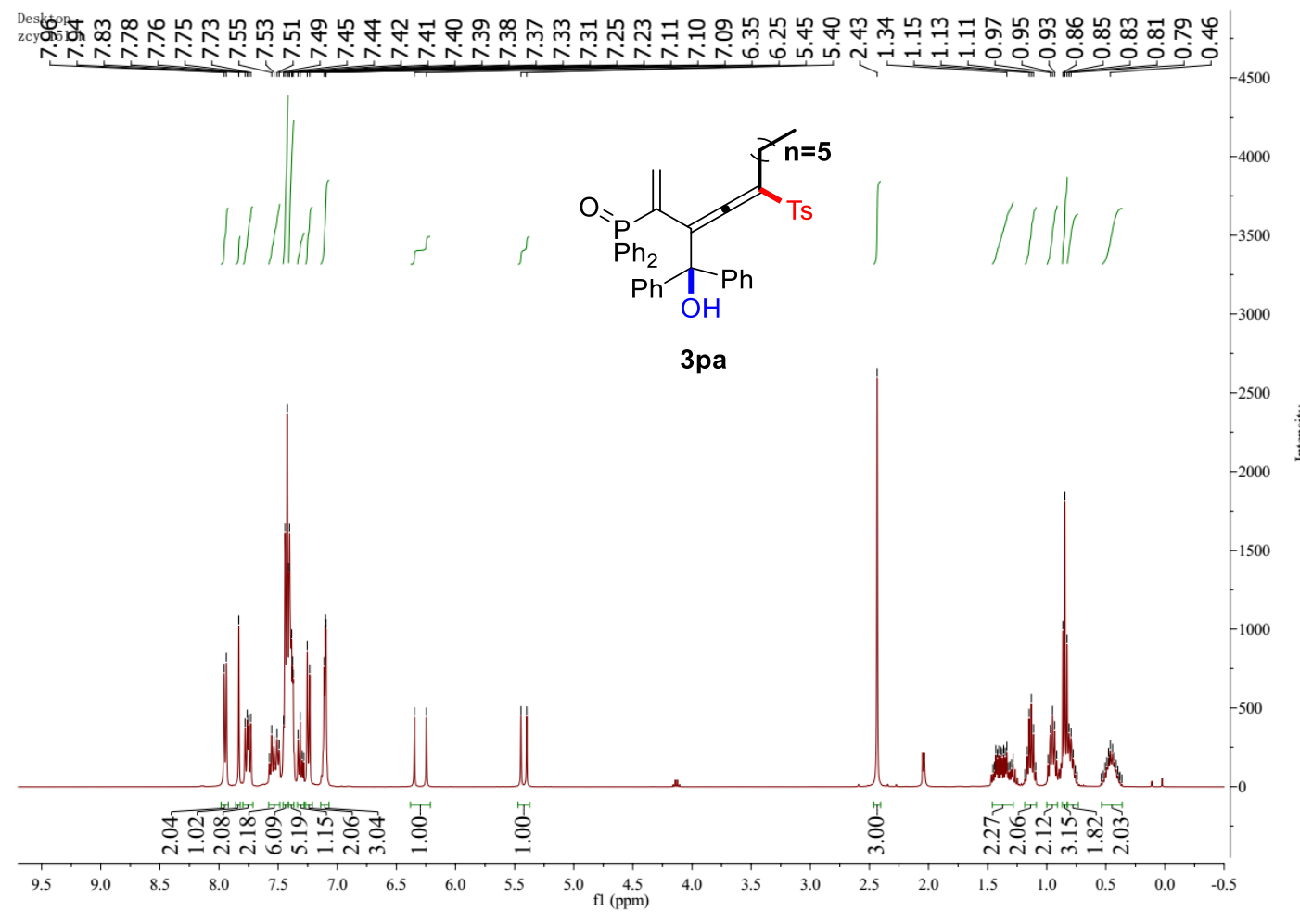

\section{${ }^{1} \mathrm{H}$ NMR $\left(400 \mathrm{MHz}, \mathrm{CDCl}_{3}\right)$ of 3pa}

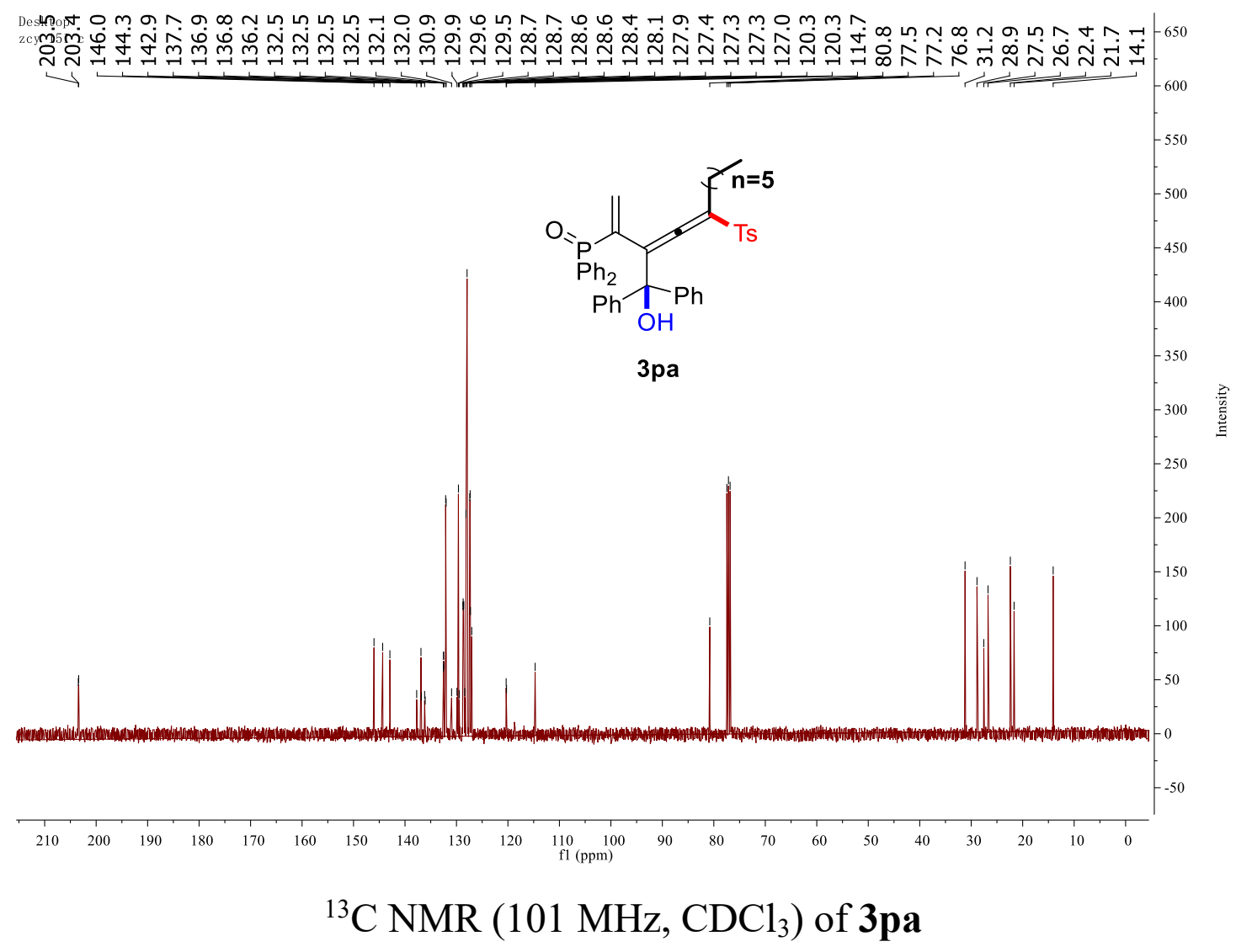




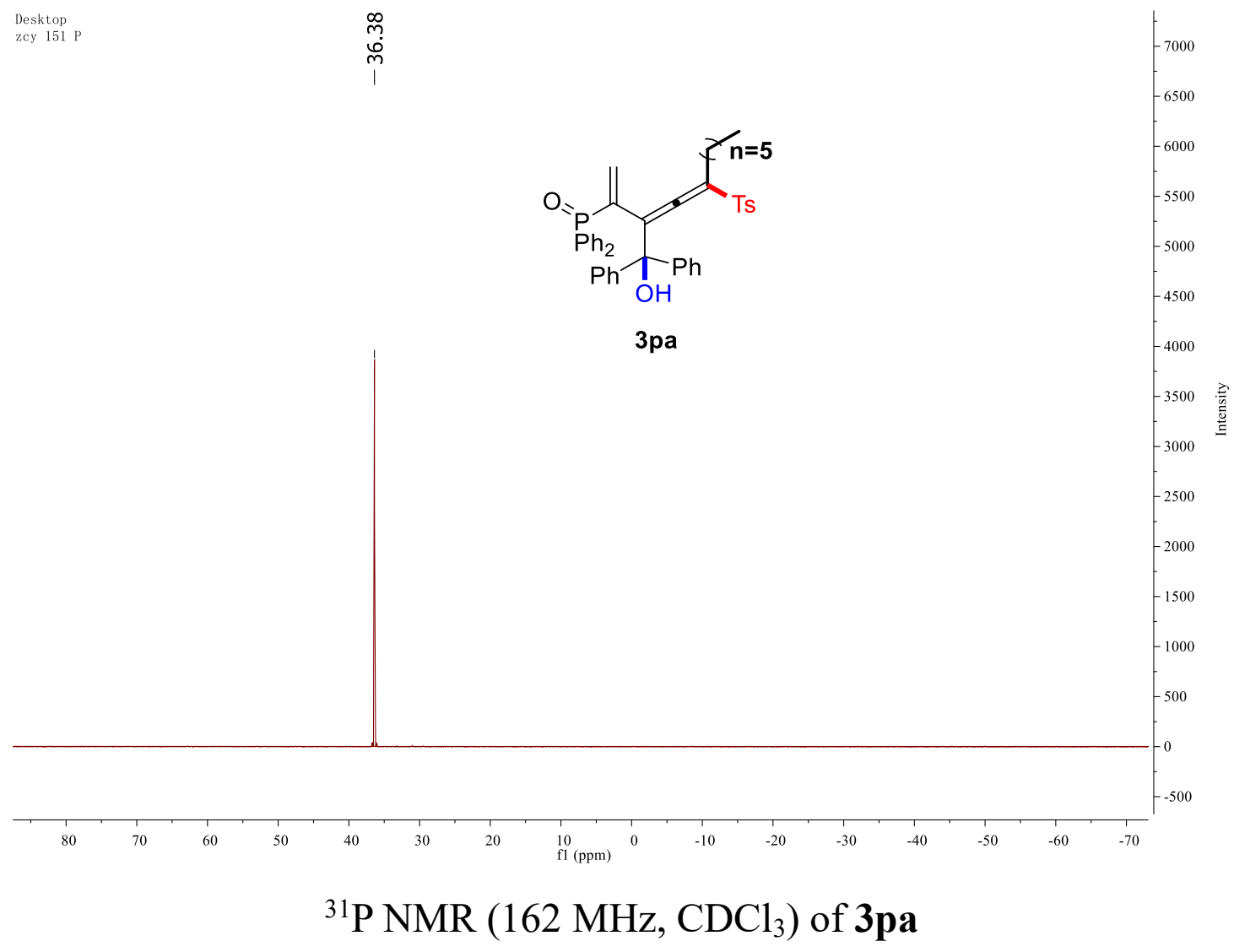

20190708-15 \#11 RT: 0.16 AV: 1 NL: 7.59E6

T: FTMS $\{1,1\}+p$ ESI Full ms [100.00-1000.00]

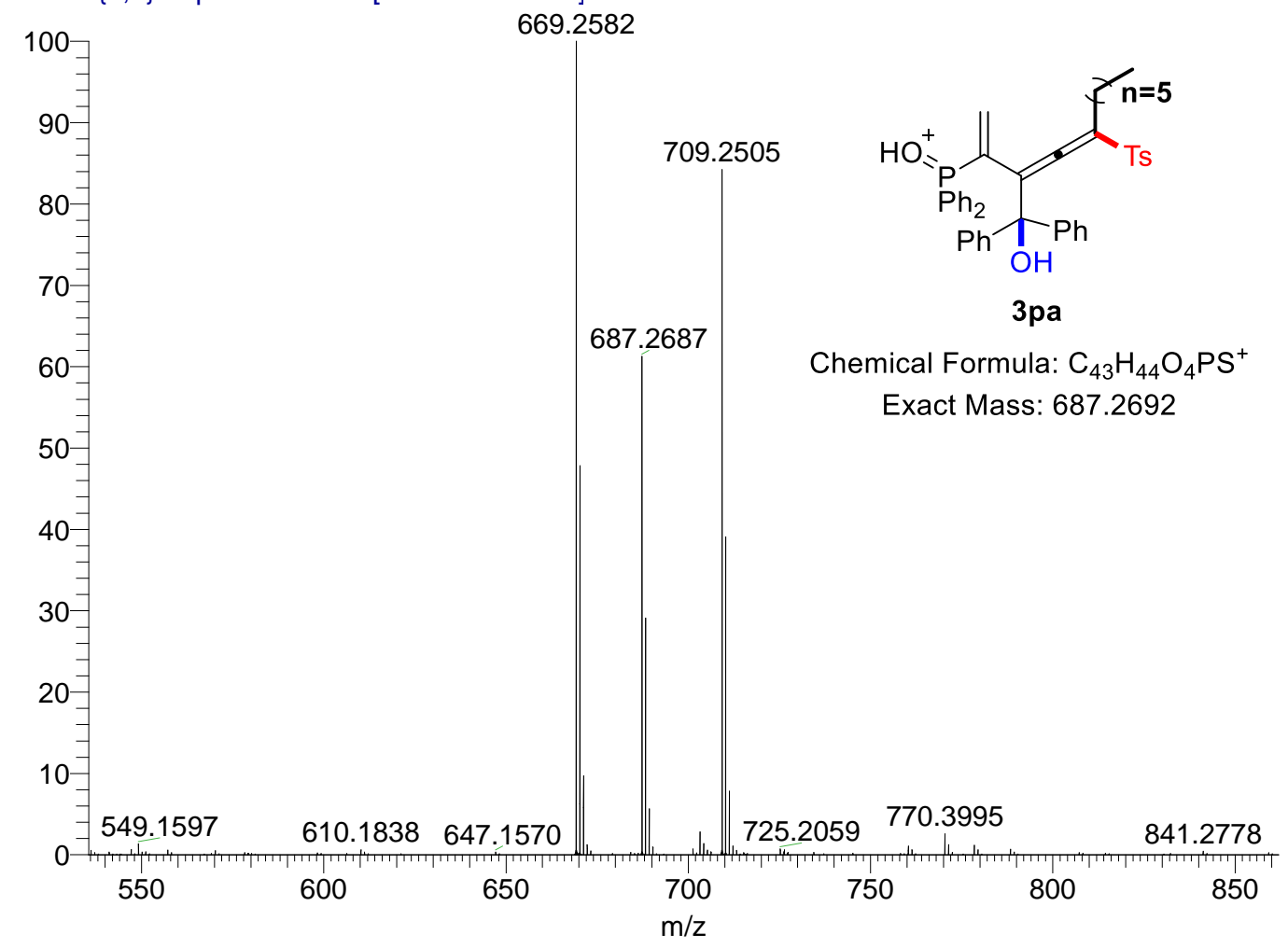




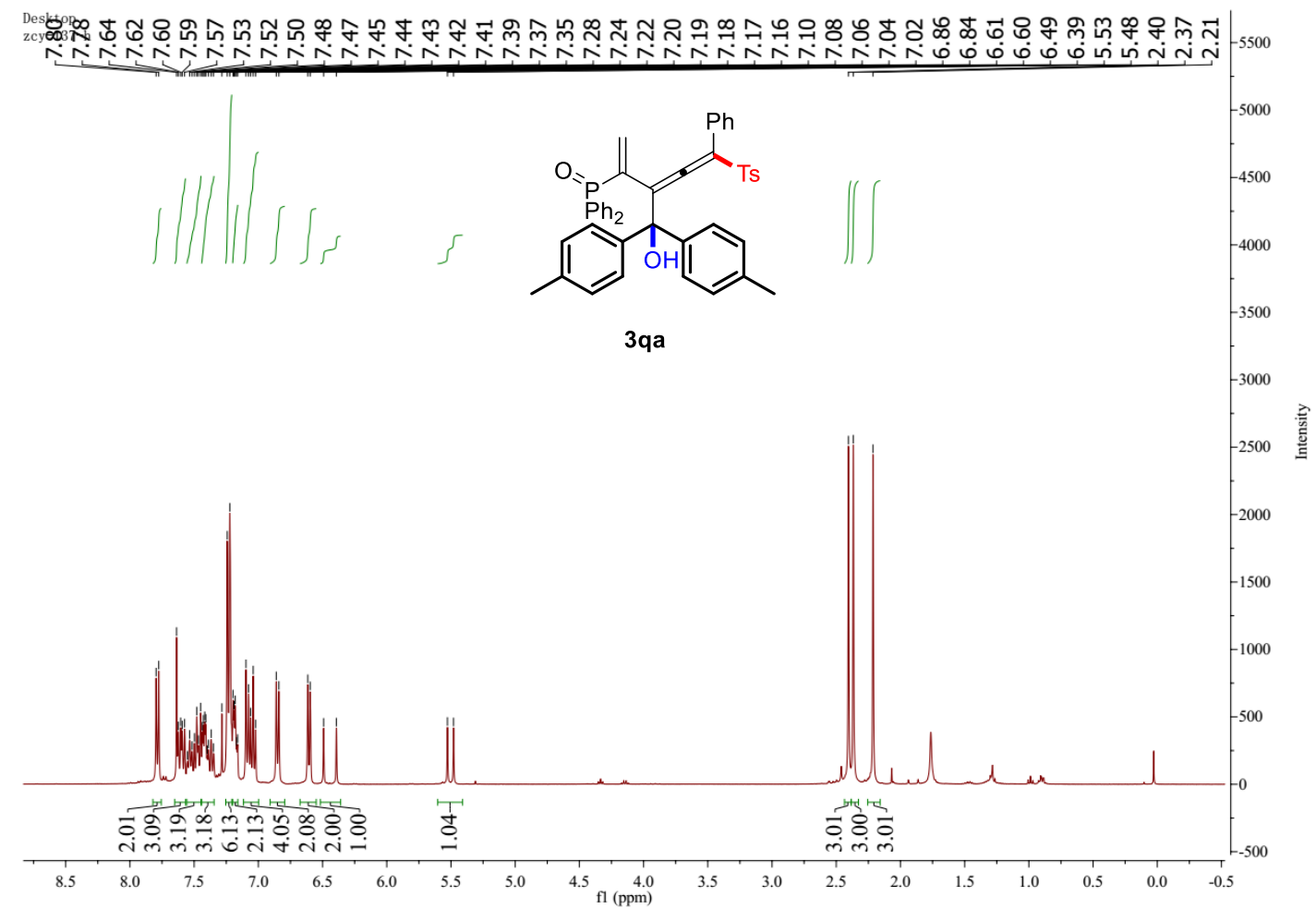

\section{${ }^{1} \mathrm{H}$ NMR $\left(400 \mathrm{MHz}, \mathrm{CDCl}_{3}\right)$ of $\mathbf{3 q a}$}

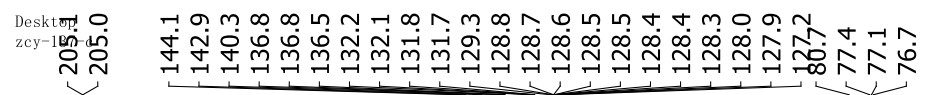

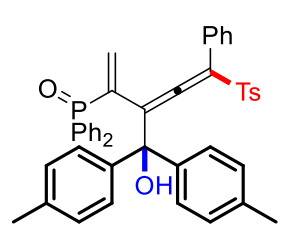

$3 q a$

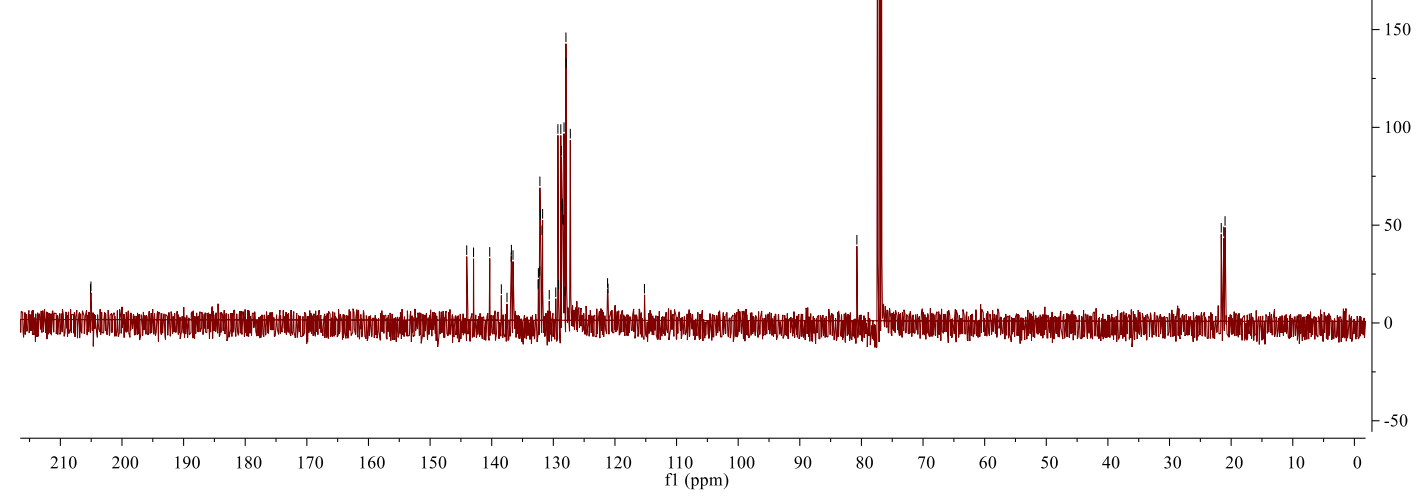

${ }^{13} \mathrm{C}$ NMR $\left(101 \mathrm{MHz}, \mathrm{CDCl}_{3}\right)$ of $\mathbf{3 q a}$ 


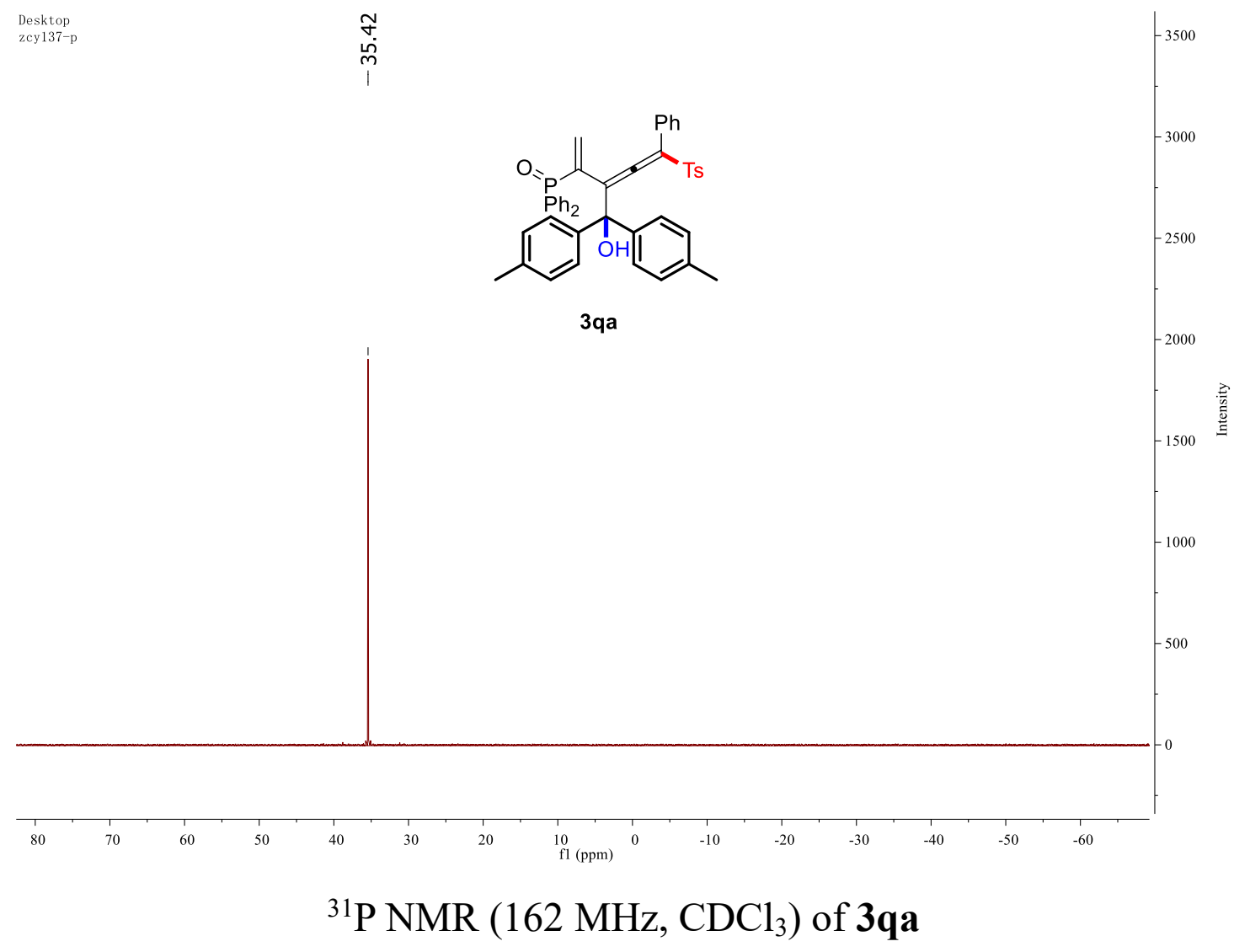

19 \#25 RT: 0.38 AV: 1 NL: $5.23 E 4$

T: FTMS $\{1,1\}+$ p ESI Full ms [100.00-1000.00]

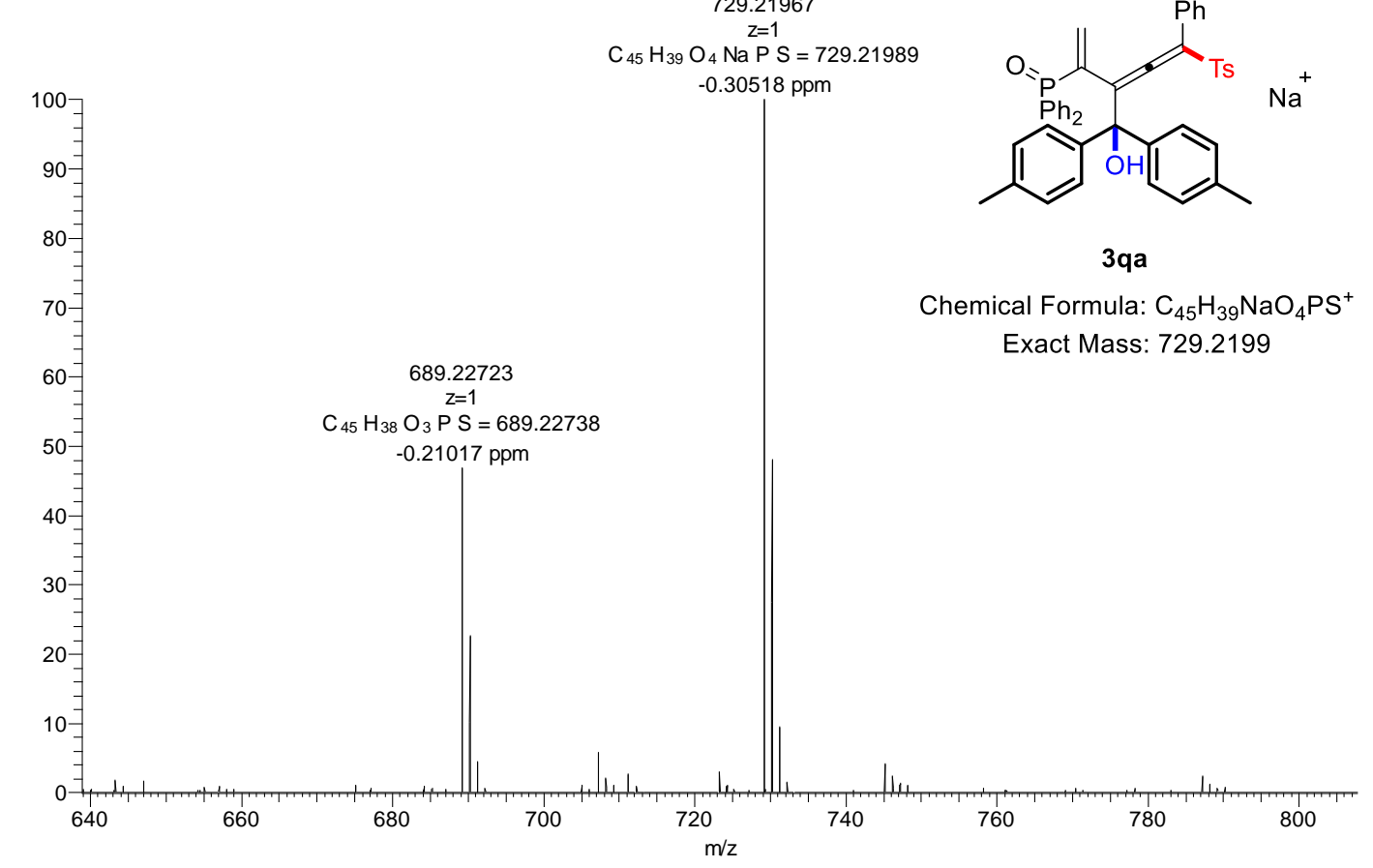




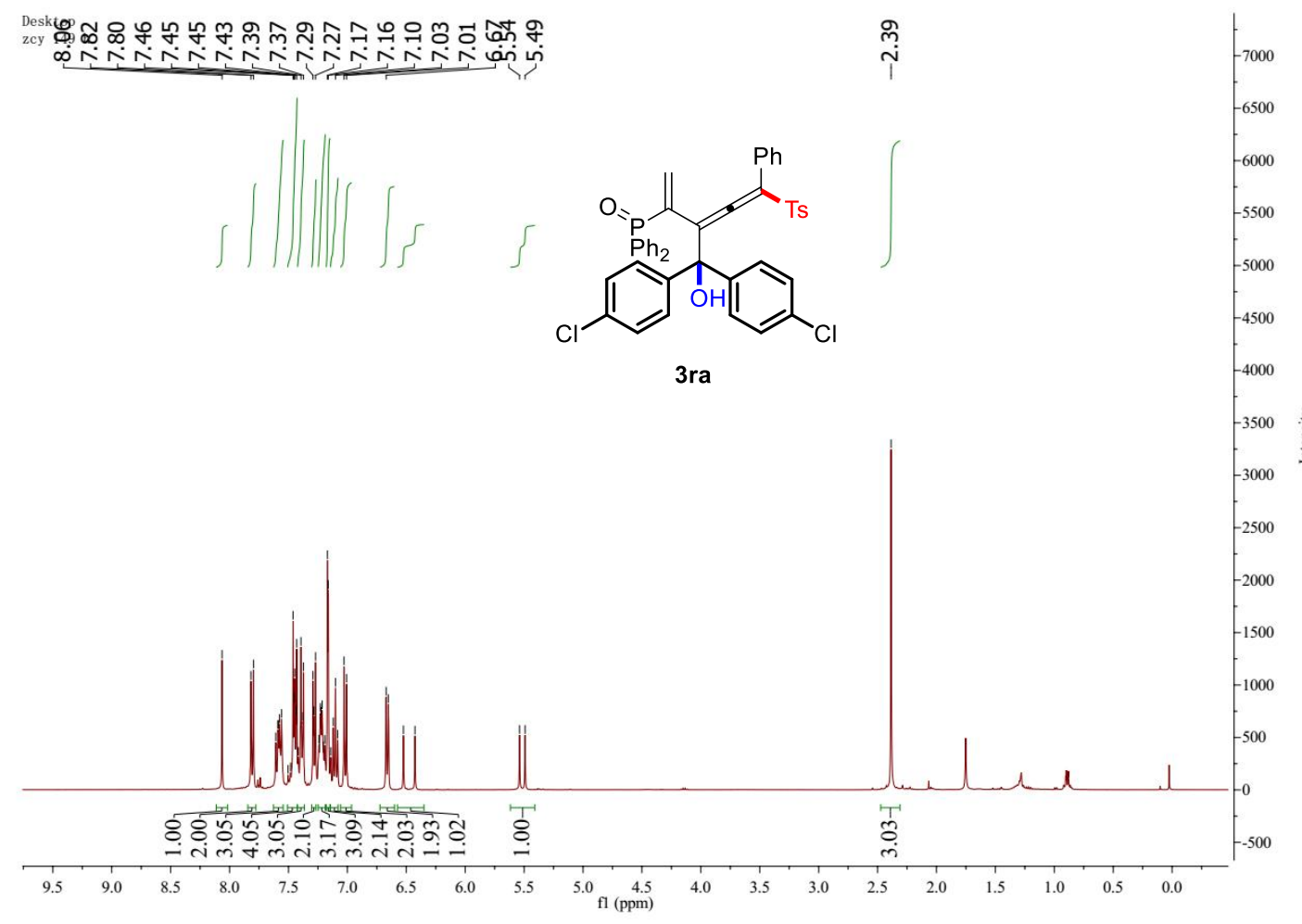

${ }^{1} \mathrm{H}$ NMR $\left(400 \mathrm{MHz}, \mathrm{CDCl}_{3}\right)$ of 3ra

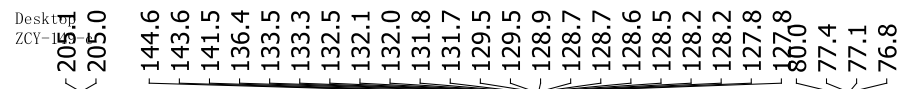

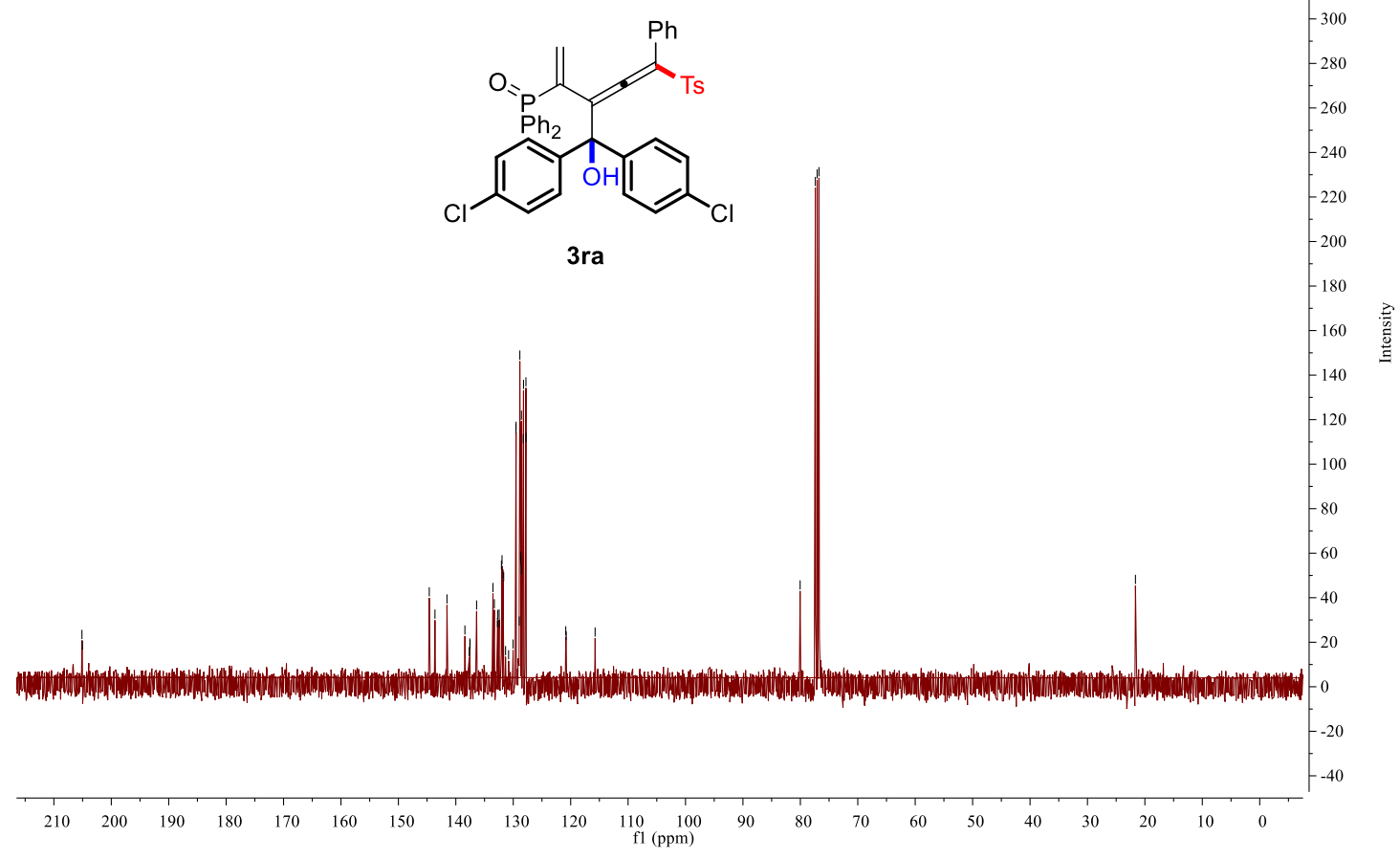

${ }^{13} \mathrm{C}$ NMR $\left(101 \mathrm{MHz}, \mathrm{CDCl}_{3}\right)$ of $\mathbf{3 r a}$ 


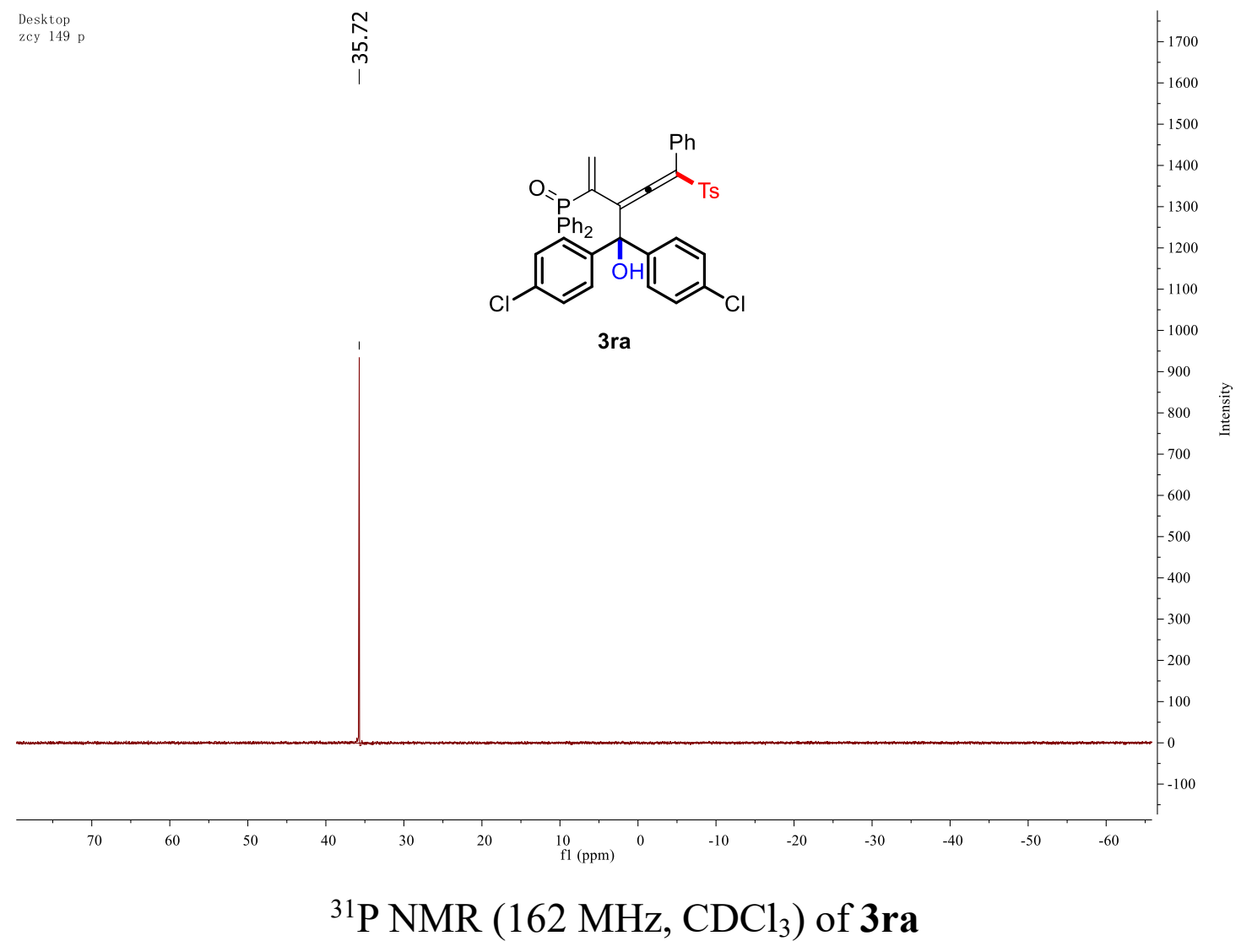

20190708-13 \#11 RT: 0.16 AV: 1 NL: 5.10E4

T: FTMS $\{1,1\}+p$ ESI Full ms [100.00-1000.00]

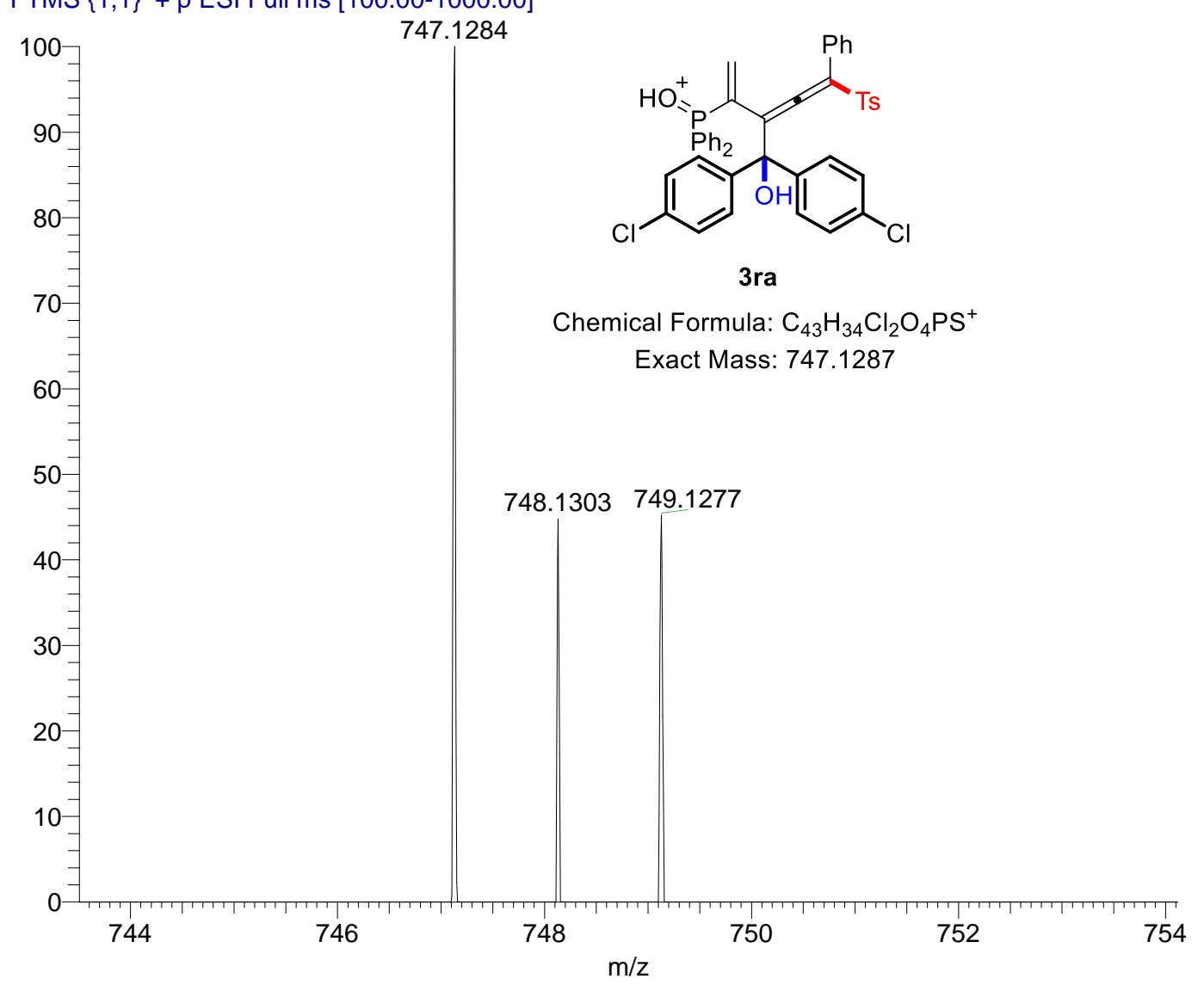




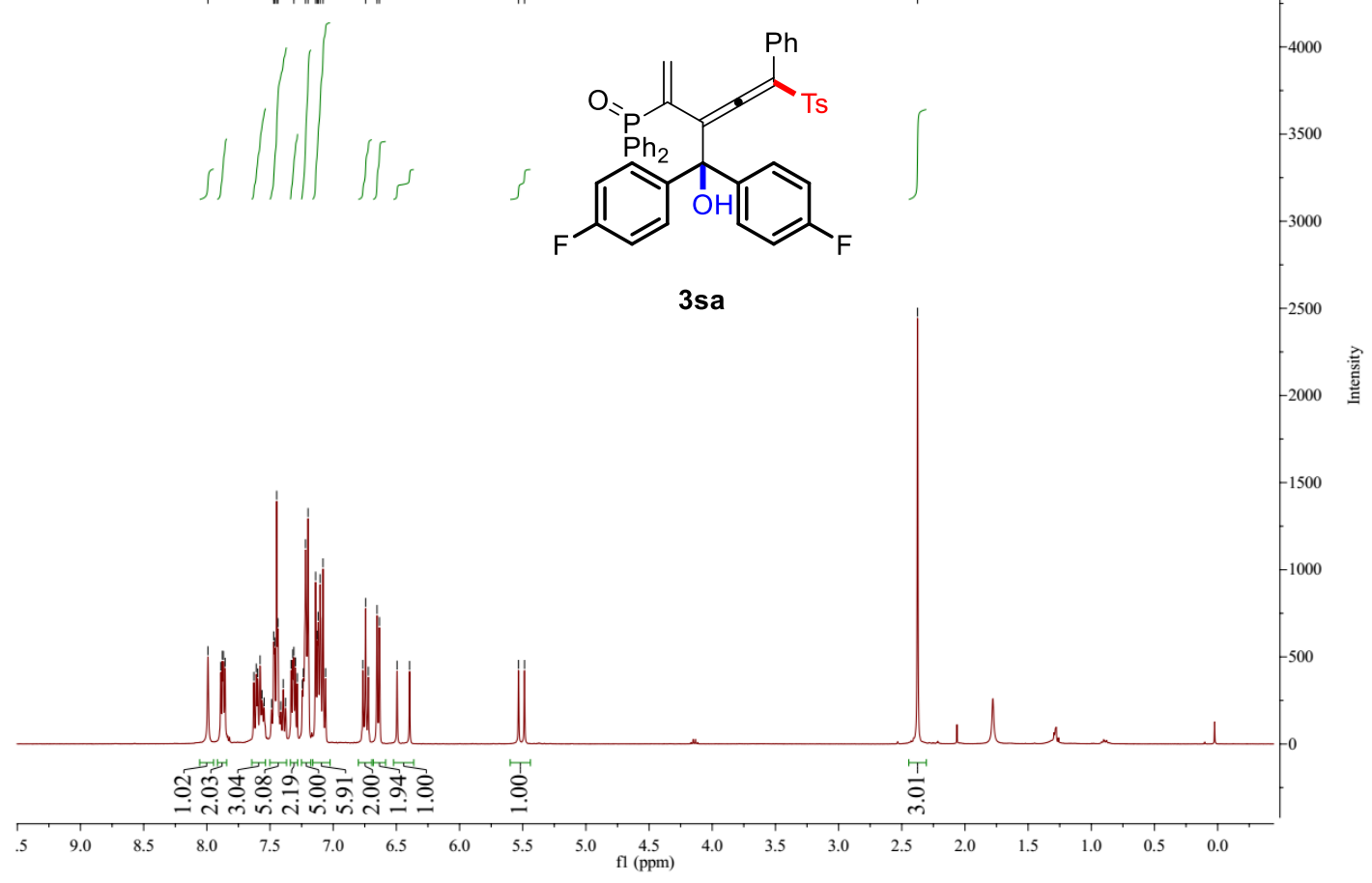

${ }^{1} \mathrm{H} \mathrm{NMR}\left(400 \mathrm{MHz}, \mathrm{CDCl}_{3}\right)$ of 3sa

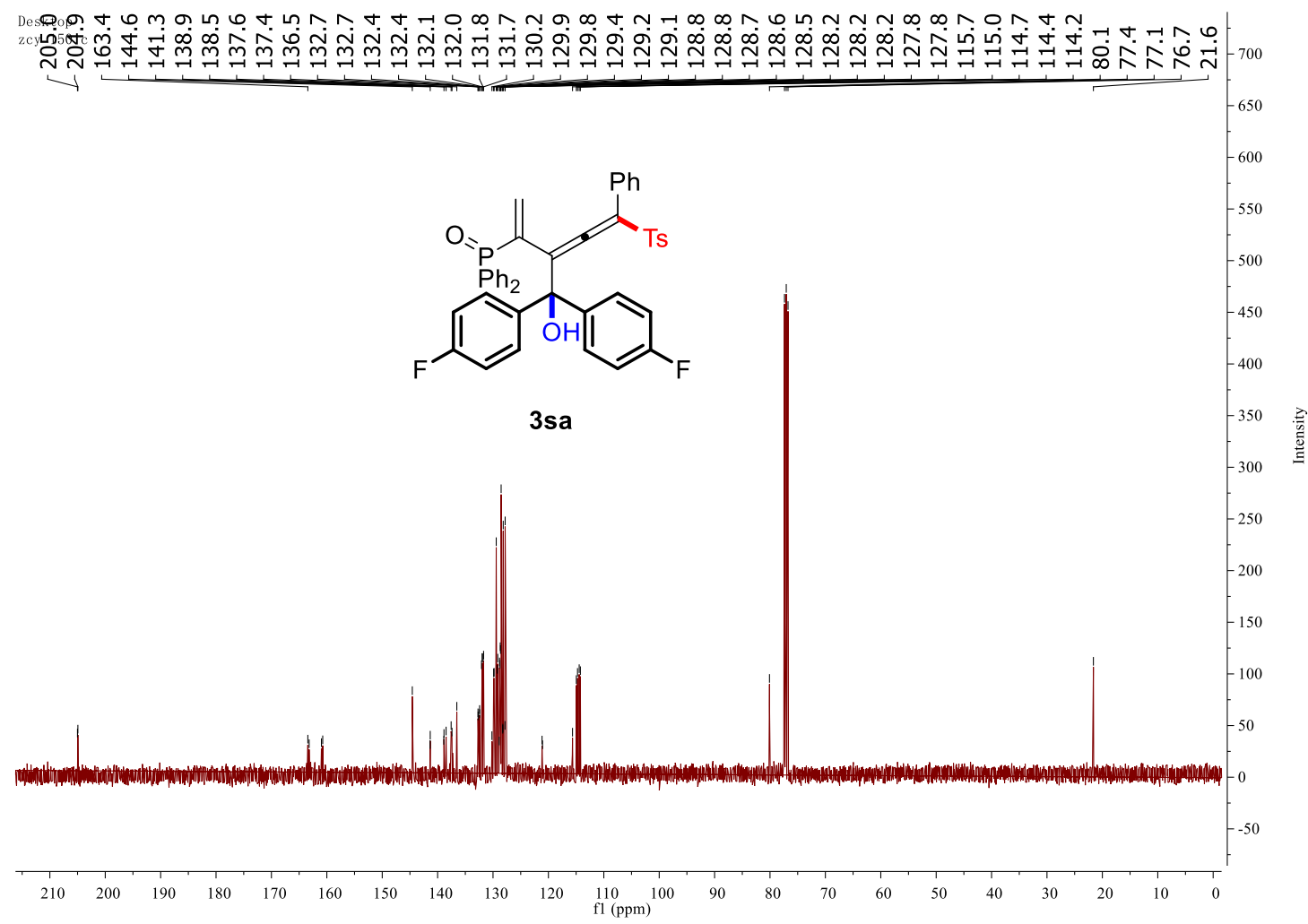

${ }^{13} \mathrm{C}$ NMR $\left(101 \mathrm{MHz}, \mathrm{CDCl}_{3}\right)$ of 3sa 


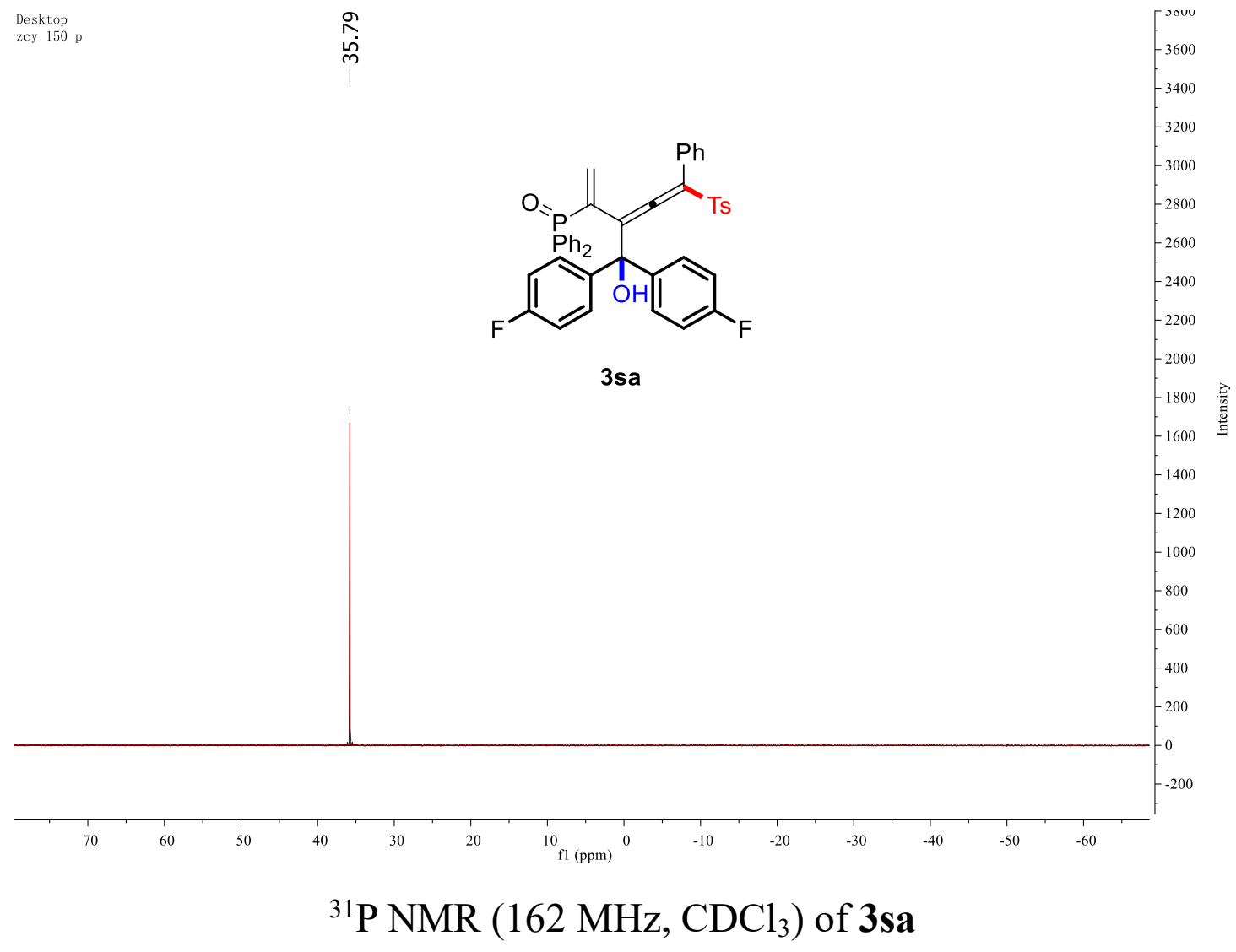

20190708-14 \#13 RT: 0.19 AV: 1 NL: 9.87E4

T: FTMS $\{1,1\}+p$ ESI Full ms [100.00-1000.00]

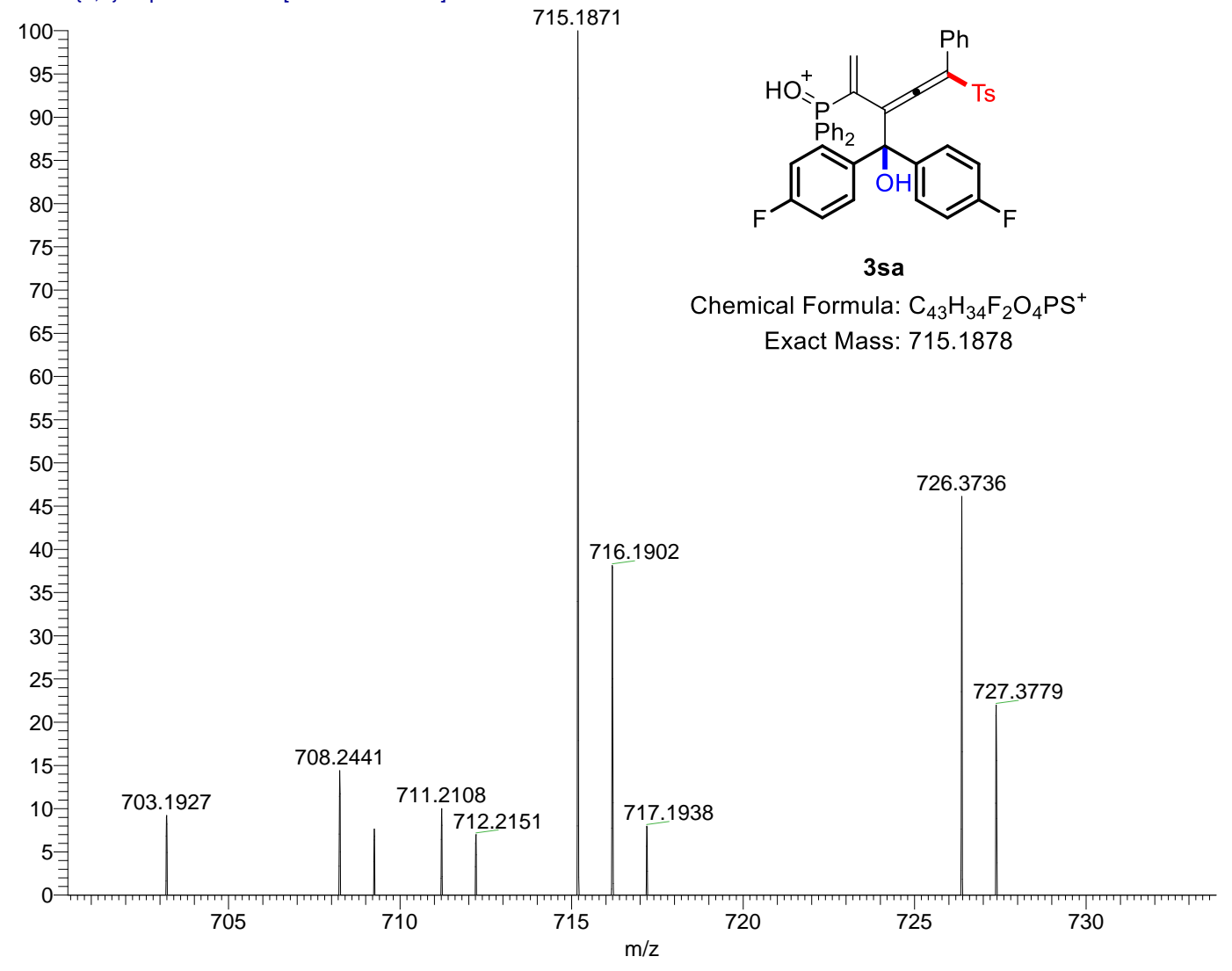




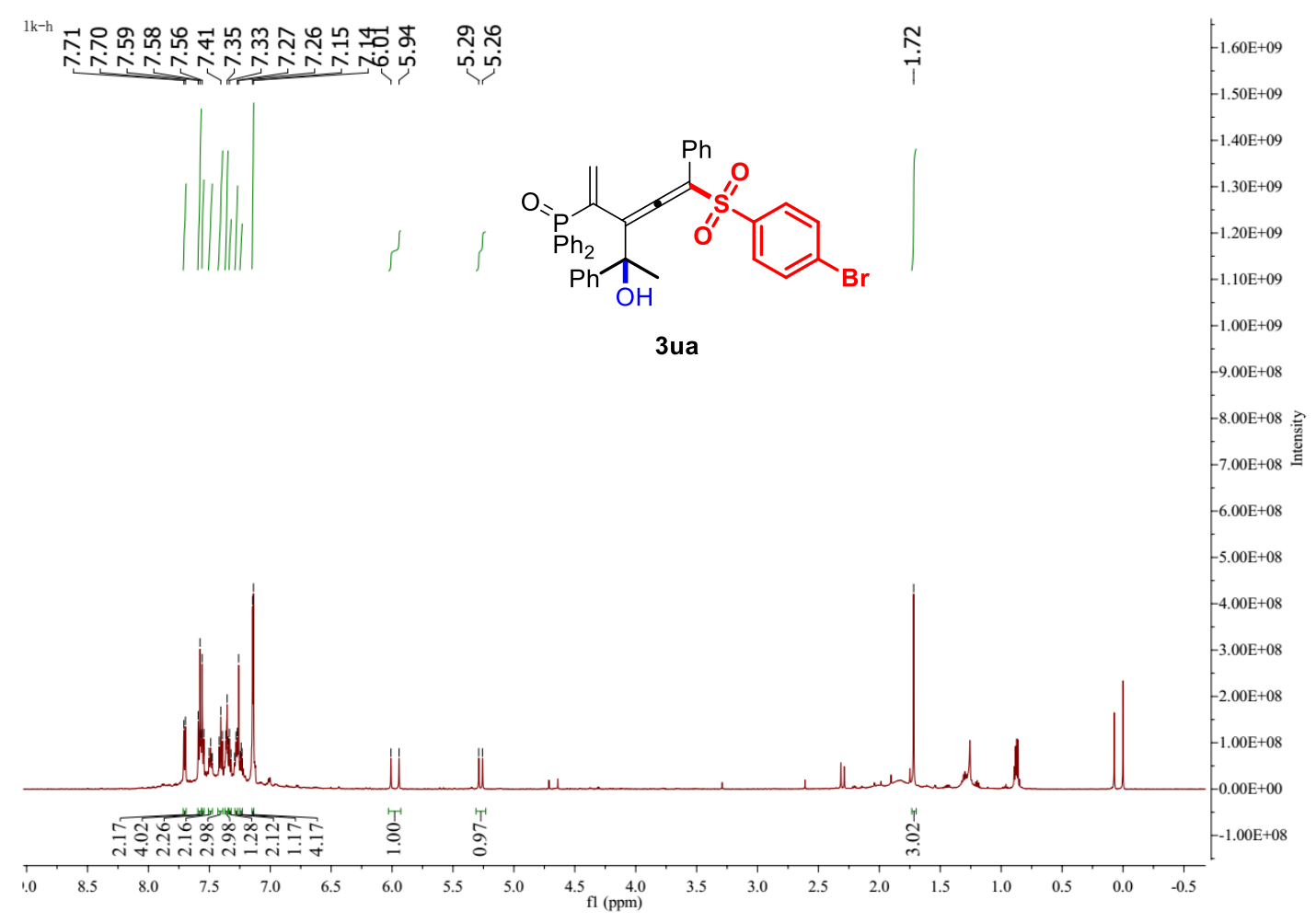

\section{${ }^{1} \mathrm{H}$ NMR $\left(600 \mathrm{MHz}, \mathrm{CDCl}_{3}\right)$ of 3 ua}

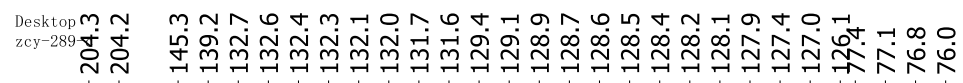

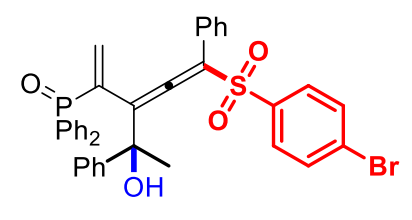

3ua

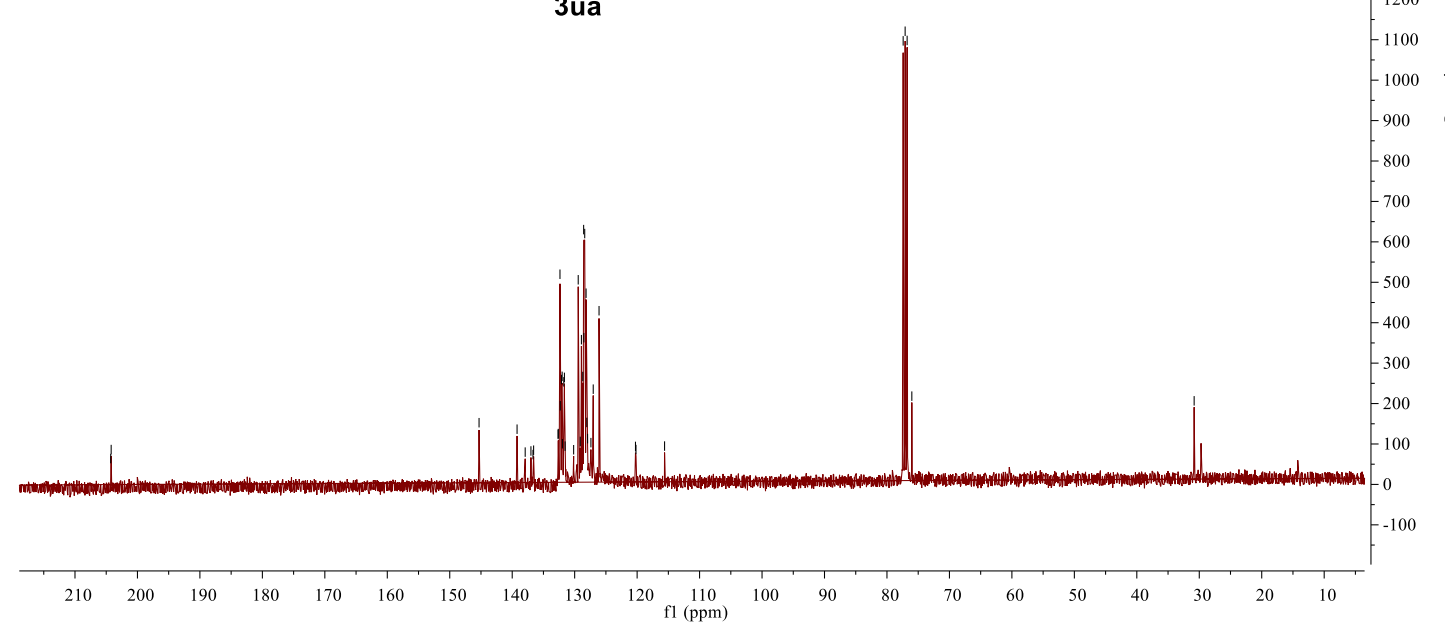

${ }^{13} \mathrm{C} \mathrm{NMR}\left(101 \mathrm{MHz}, \mathrm{CDCl}_{3}\right)$ of 3ua 


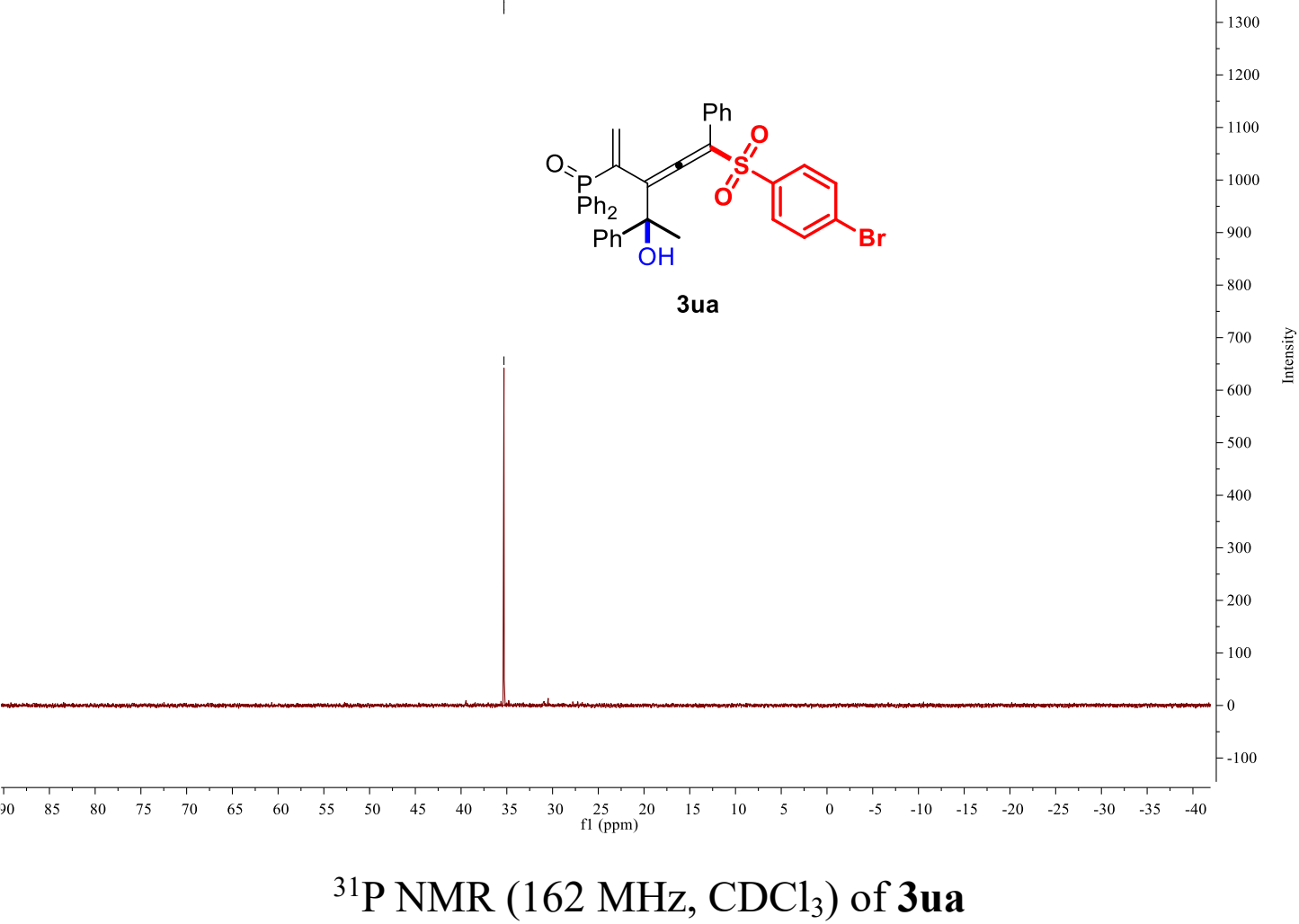

20200827-10 \#29 RT: 0.44 AV: 1 NL: 1.23E5

T: FTMS $\{1,1\}+p$ ESI Full ms [100.00-1000.00]

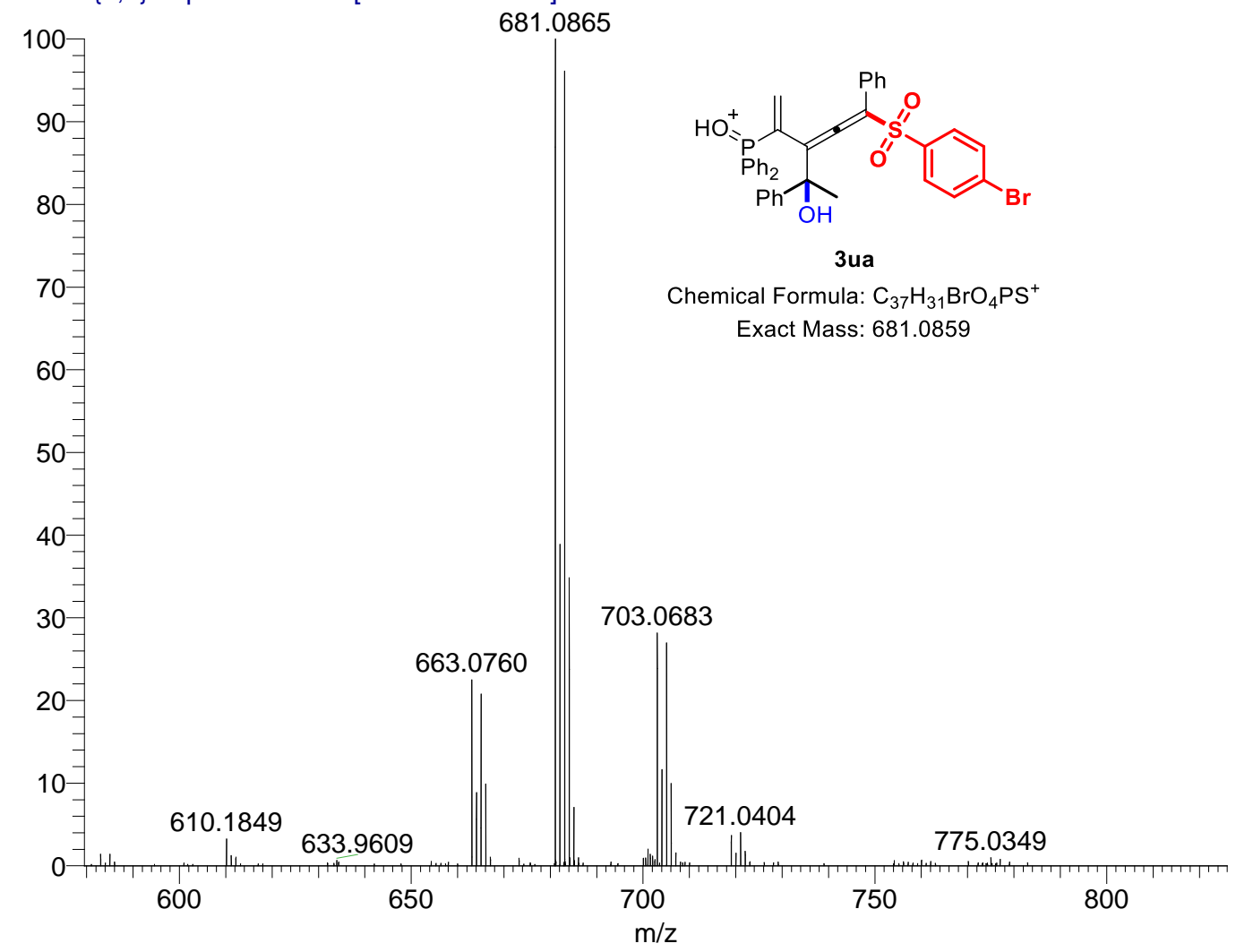



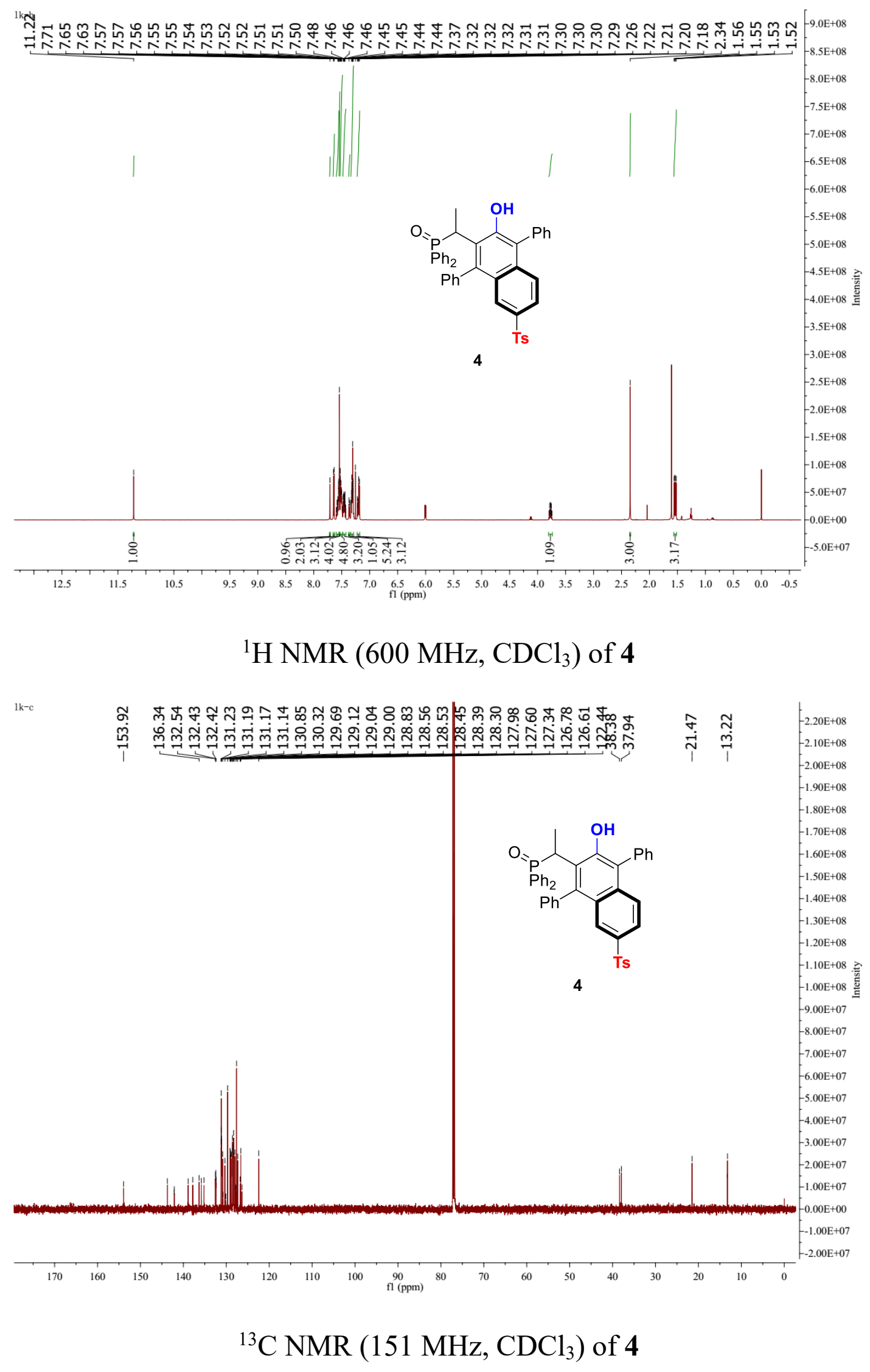


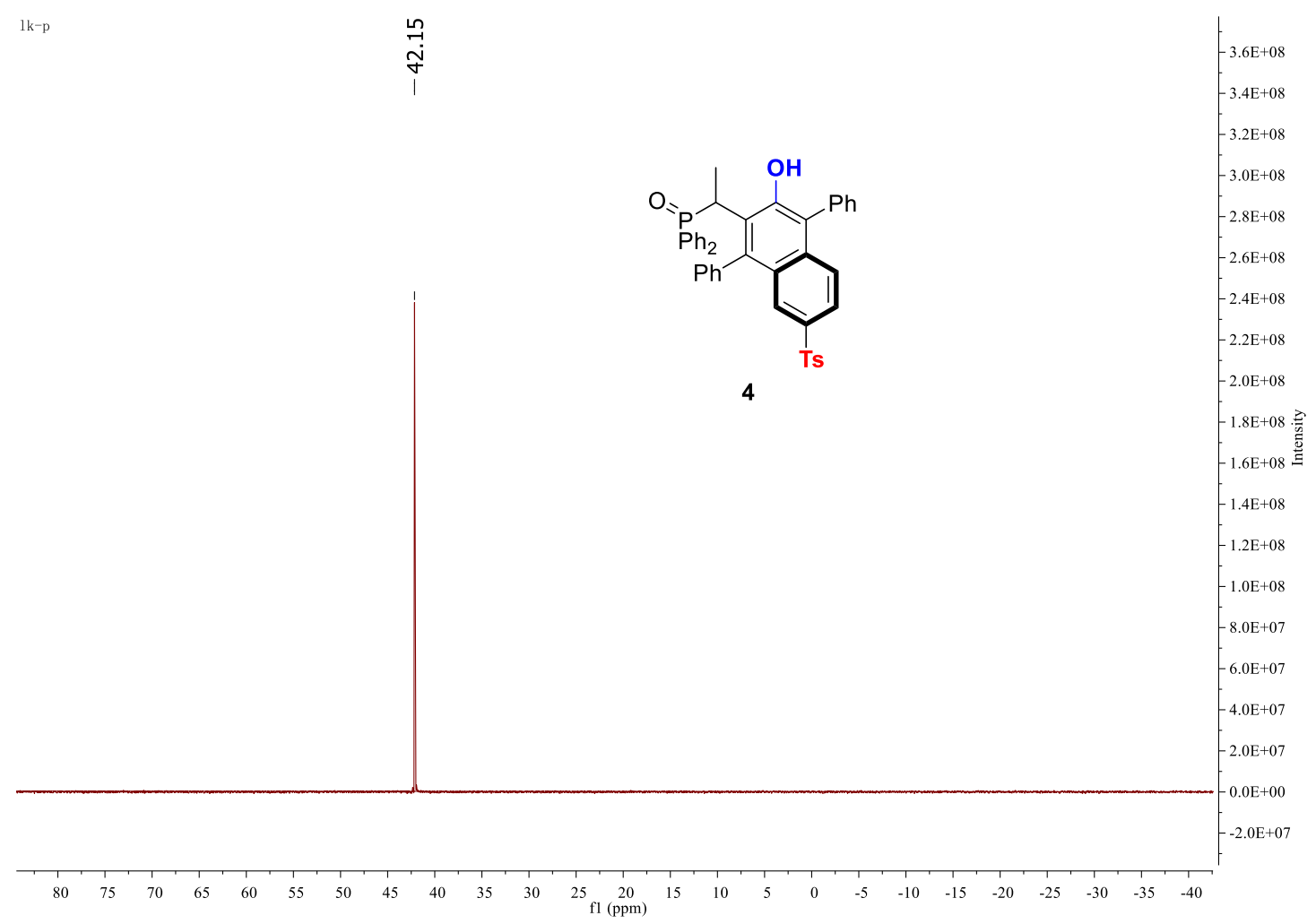

\section{${ }^{31} \mathrm{P}$ NMR $\left(243 \mathrm{MHz}, \mathrm{CDCl}_{3}\right)$ of 4}

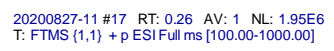

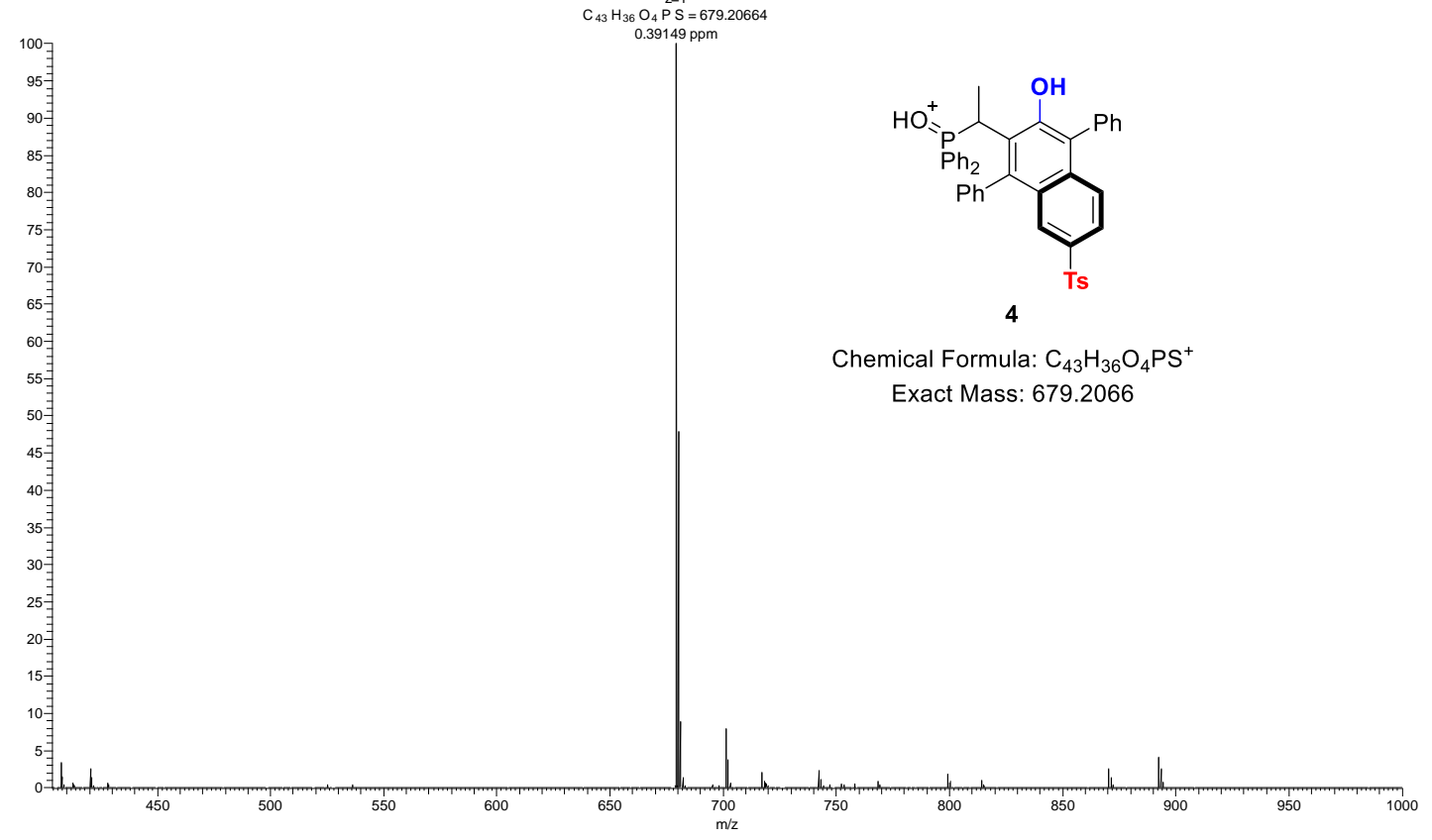




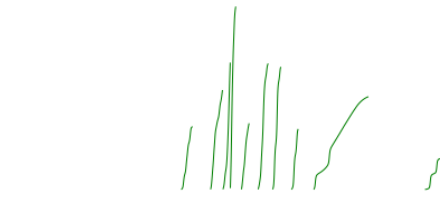<smiles>C=C(P=O)C(C(Cl)=C(P)[As])=C(c1ccccc1)c1ccccc1</smiles>

5a

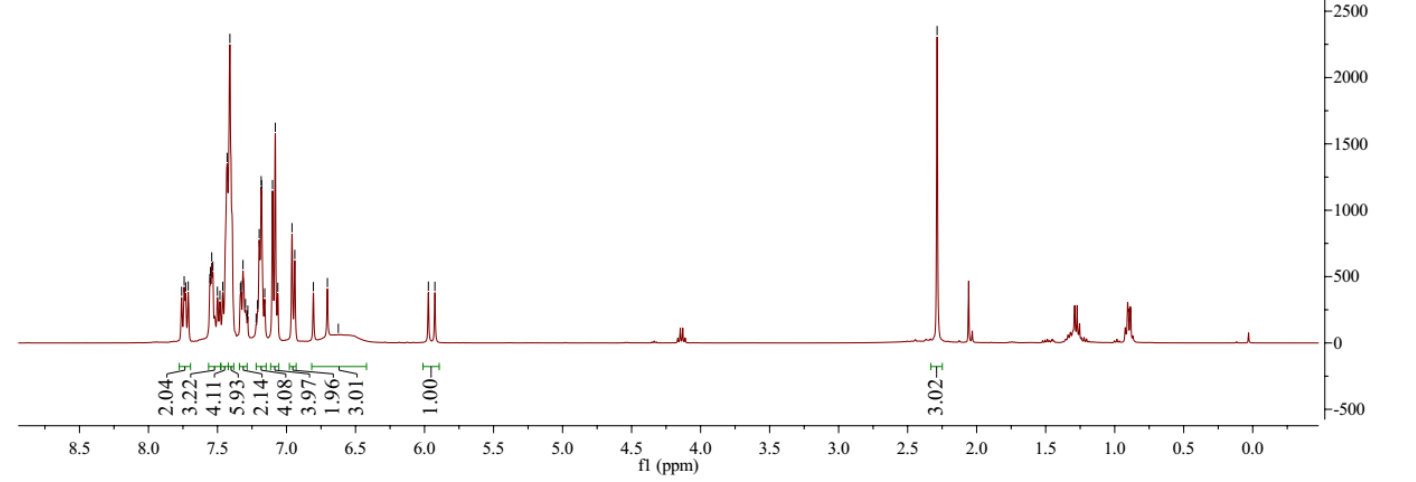

${ }^{1} \mathrm{H}$ NMR (400 MHz, $\mathrm{CDCl}_{3}$ ) of $\mathbf{5 a}$

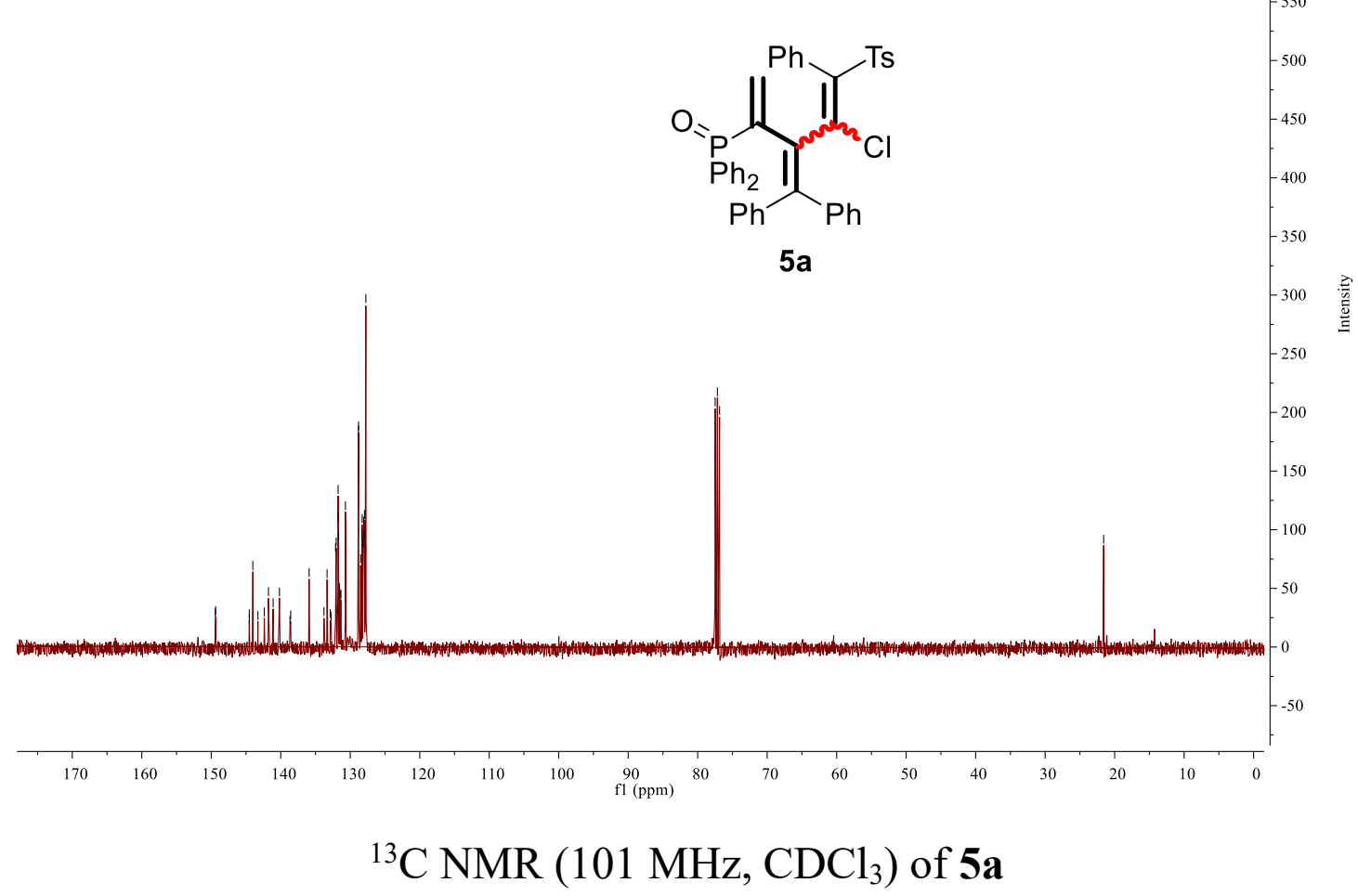




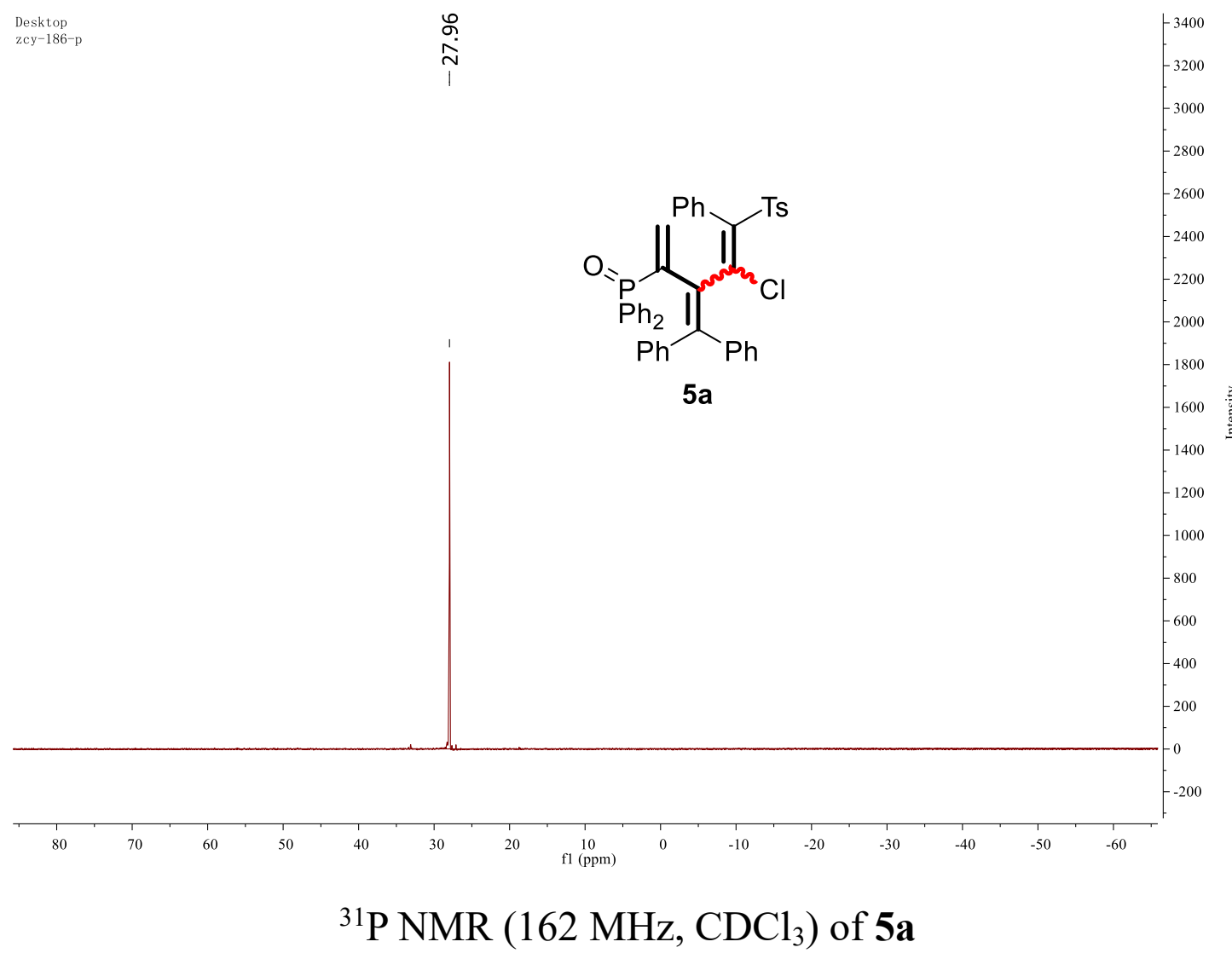

20210118-3 \#19 RT: 0.23 AV: 1 NL: 4.60E5 T: FTMS $\{1,1\}+p$ ESI Full ms [100.00-1000.00]

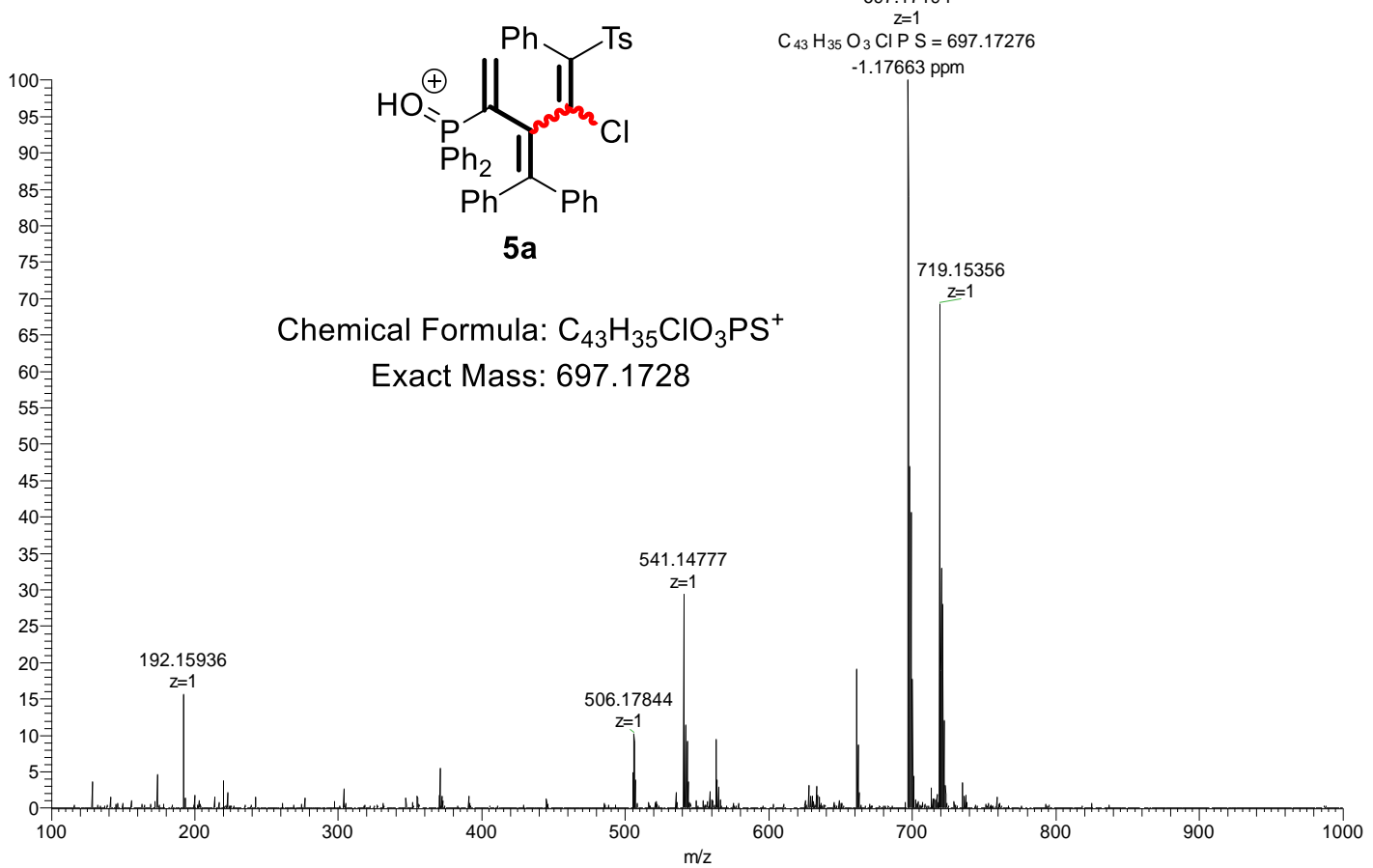



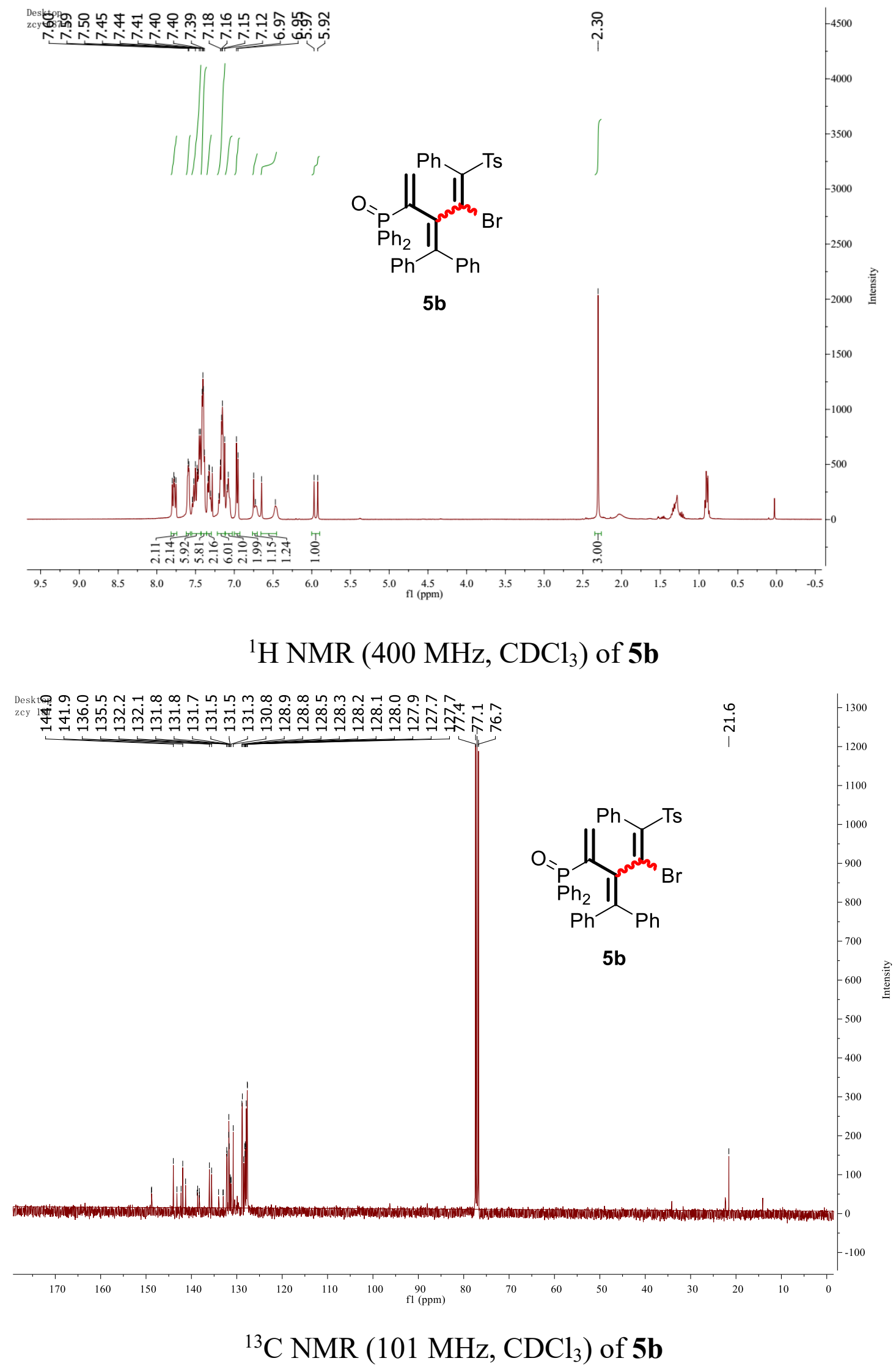


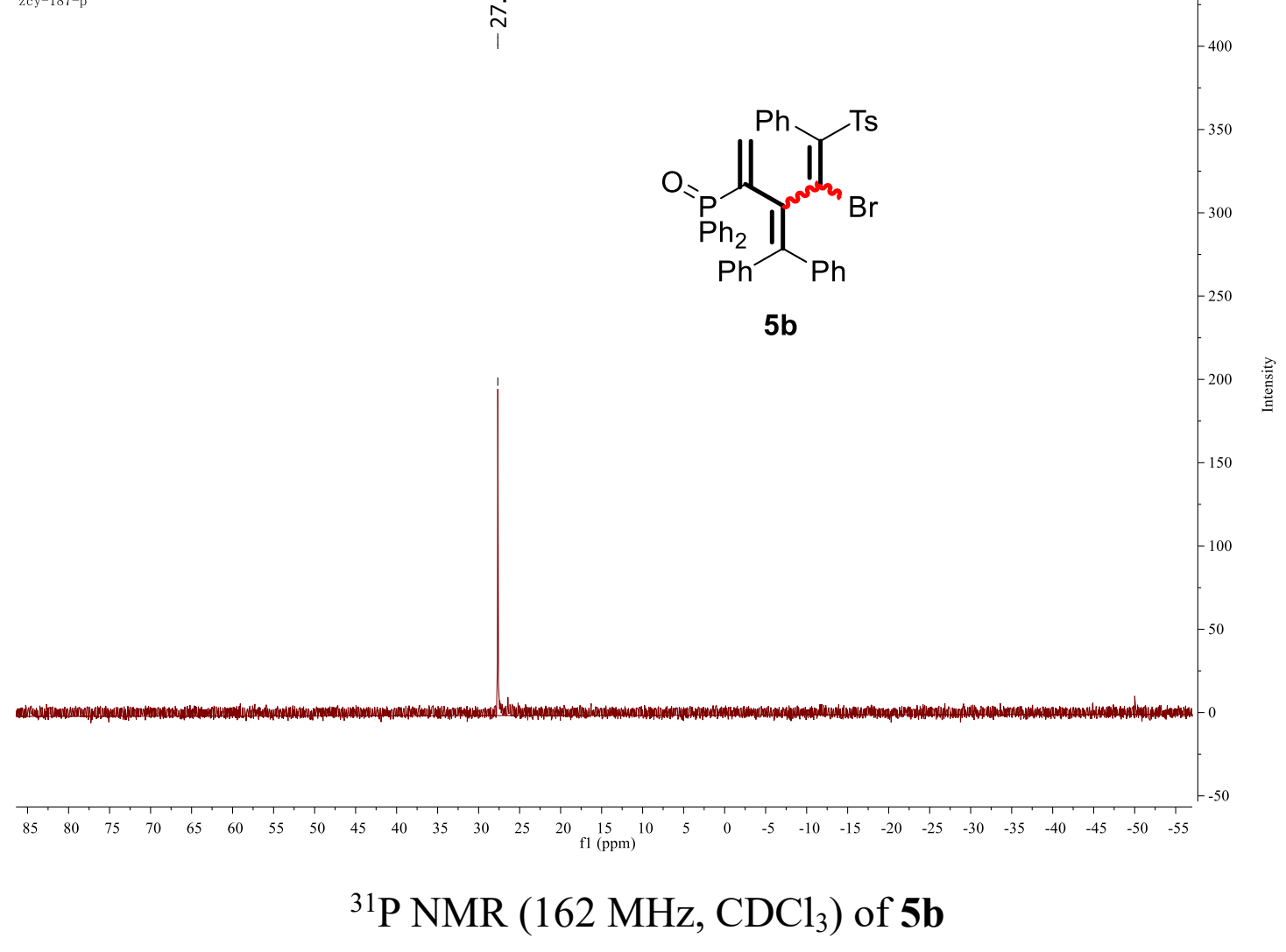

20210118-4 \#23 RT: 0.28 AV: 1 NL: $1.01 E 5$ T: FTMS $\{1,1\}$ + p ESI Full ms [100.00-1000.00]

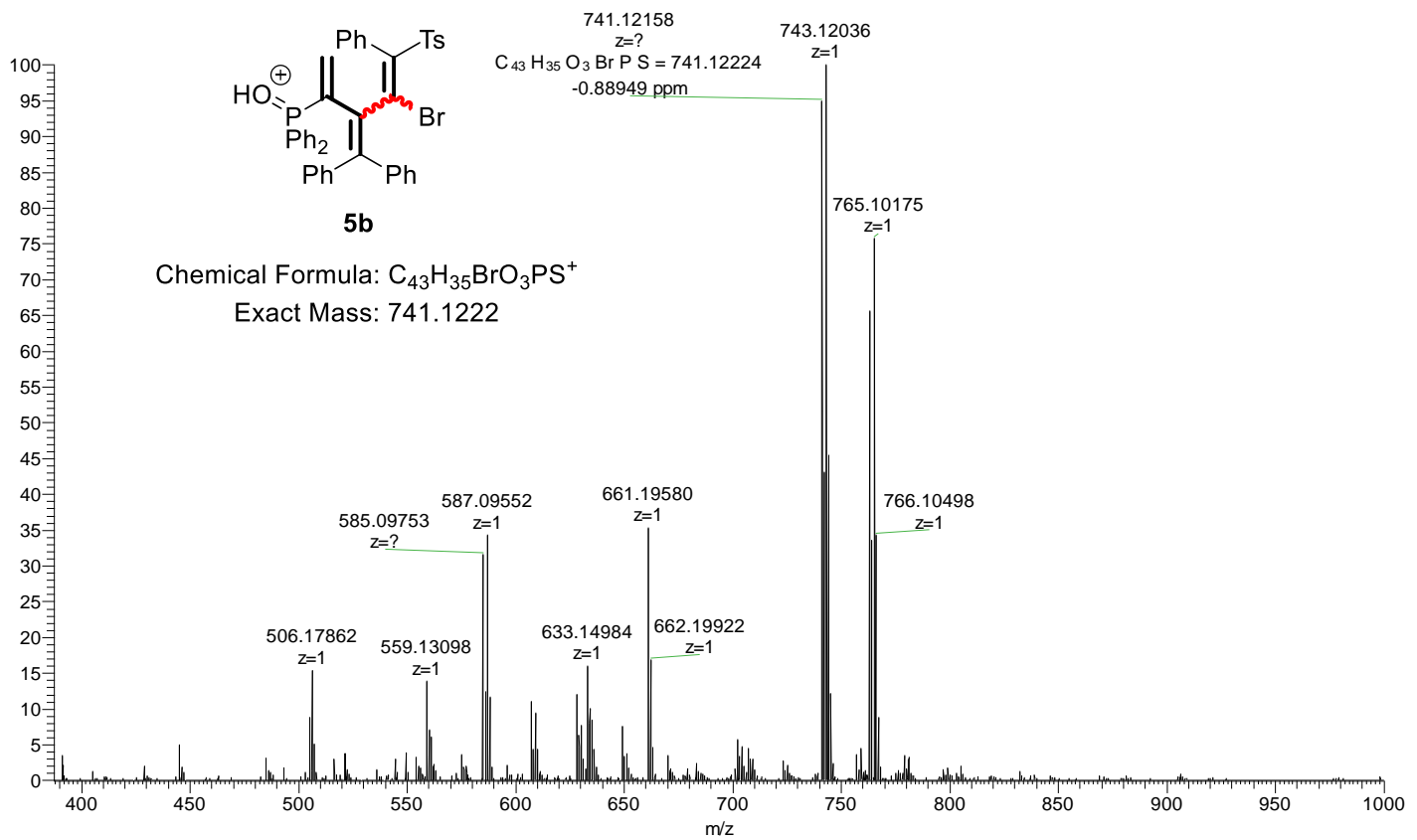

